This report was prepared as an account of work sponsored by an agency of the United States Government. Neither the United States Government not any agency thereof, nor any of their emplayees, makes any wartanty, express or implied, or assumes any legal liability or responsibility for the accuracy, completeness, or usefulness of any information, apparatus, product, or process disc: $\therefore$ d, or represents that its use wou!d not infringe privately owned rights. Reference herein to any specific commercial product. process, or service by trade name, trademark. manufacturer, or otherwise does not necessarily constitute or imply its endorsement, recommendation, or favoting by the United Siates Government or any agency thereof. The views and opinions of authors expressed herein do not necessarily state or reflect those of the United States Government or any agency thereof.

UCRL- -53881

DE89 000664

\title{
Hot-Electron Plasma Formation and Confinement in the Tandem Mirror Experiment-Upgrade
}

\author{
D. B. Ress
}

(Ph.D. Thesis)

\author{
A Dissertation \\ Submitted to \\ the Department of Electrical Engineering \\ and Committee on Graduate Studies \\ of Stanford University \\ in Partial Fulf Iment of the Requirements \\ for the Degree of \\ Doctor of Philosopiny
}

\section{Manuscript date: June 1988}

\section{LAWRENCE LIVERMORE NATIONAL LABORATORY University of California - Livermore, California - 94550}




\section{Contents}

1 Introduction 1

1.1 The Tandem Mirror Experiment-Upgrade . . . . . . . . . . 2

1.1.1 Physical Description . . . . . . . . . . . . . 5

1.1 .2 ECRF Heating System . . . . . . . . . . 8

1.2 Features of a Typical TMX-U Discharge . . . . . . . . 10

1.2 .1 Plasma Line-Density . . . . . . . . . . . . . 10

1.2 .2 Diamagnetism . . . . . . . . . . . . . 12

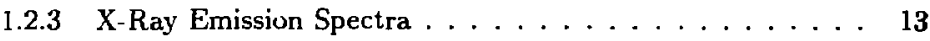

1.3 Overview ........................ 15

2 The Hot-Electron Energy/Density Probe 22

2.1 Physical Description . . . . . . . . . . . . . . 22

2.2 HEED Probe Electronics . . . . . . . . . . . . . . . . 24

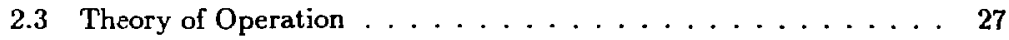

2.4 Calorimetry ........................ 34

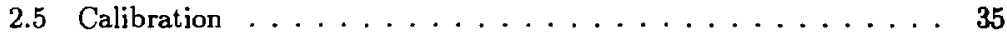

3 Hot-Electron Plasma Formation $\quad \mathbf{4 0}$

3.1 Equilibrium Plasma Spatial Structure . . . . . . . . . . . 40

3.1.1 Limiter Probe Scans . . . . . . . . . . . . . . . 41

3.1.2 Perpendicular Pressure Profile ... . . . . . . . . . 44

3.1.3 Comparison of the Magnetic-Curvature Drifts with the Pressure Profile . . . . . . . . . . . . . . . 51

3.1.4 Spatial Structure of the Super-Hot Component . . . . . 53

3.1.5 Spatial Structure of the Hot Component . . . . . . . . 65

3.1.6 The Cold and Warm Plasma Components . . . . . . . . . 66

3.1.7 Summary of the Plasma Structure . . . . . . . . 70

3.2 Initial Hot-Electron Plasma Formation . . . . . . . . . . . 74

3.3 Effect of the Midplane Magnetic Field on Hot-Electron Plasma Formation . . . . . . . . . . . . . . . . 81

3.3.1 Vacuum Magnetic-Field Variation Experiment . . . . . . 81

3.3 .2 Data . . . . . . . . . . . . . . . . 82

3.3 .3 Analysis ......................... 85 
4 Hot-Electron Plasma Confinement $\quad 00$

4.1 ECRF A bsorption . . . . . . . . . . . . . . . 91

4.1.1 ECRF Absorption Diagnostics . . . . . . . . . . 92

4.1 .2 Temporal Behavior . . . . . . . . . . . . . . . 94

4.1 .3 Spatial Structure . . . . . . . . . . . . . . . 96

4.1.4 Variation with Vacuum Magnetic Field . . . . . . . . 96

4.1.5 Comparison with Theory . . . . . . . . . . . 99

4.2 Energy Storage . . . . . . . . . . . . . . . 111

4.2 .1 Temporal Behavior . . . . . . . . . . . . . . 111

4.2 .2 Spatial Structure . . . . . . . . . . . . . . 115

4.2.3 Variction with Vacuum Magnetic Field . . . . . . . 115

4.3 Axial Losses . . . . . . . . . . . . . . . . . . . . . . . 117

4.3.1 Axial Loss Diagnostics . . . . . . . . . . . . . . 117

4.3.2 Axial End-Loss Characteristics . . . . . . . . . . . 118

4.3 .3 Temporal Variation . . . . . . . . . . . 119

4.3.4 Spatial Structure . . . . . . . . . . . . . . . . 122

4.3.5 Variation with Vacuum Magnetic Field . . . . . . . 122

4.4 Radial Losses . . . . . . . . . . . . . . . . . . . . . . . . . 127

4.4.1 Temporal Variation . . . . . . . . . . . . . . . 128

4.4 .2 Spatial Structure . . . . . . . . . . . . . . 130

4.4.3 Variation with Vacuum Magnetic Field . . . . . . . . 130

4.4 Radial Confinement . . . . . . . . . . . . . 133

4.5 ECRH Power Balance . . . . . . . . . . . . . . . 139

4.5 .1 Temporal Variation . . . . . . . . . . . . . 139

4.5.2 Variation with Vacuum Magnetic Field . . . . . . . . 140

5 Conclusions $\quad 144$

5.1 Hot-Electron Plasma Formation . . . . . . . . . . . . . . . . . 144

5.2 Hot-Electron Plasma Confinement . . . . . . . . . . . 145

5.3 Implications for Thermal-Barrier Operation . . . . . . . . 146

5.4 Suggestions for Future Work . . . . . . . . . . . . 148

A Computer Code Descriptions $\quad 150$

A.1 The SPLINE Guiding Center Drift Code . . . . . . . . . 150

A.2 The MECH Power Deposition Code . . . . . . . . . 151

B Electron Radial Transport $\quad 154$ 


\section{List of Tables}

1.1 Typical parameters of the energy distribution components. . . . . 16

2.1 Summary of HEED probe calibration parameters . . . . . . 37

3.1 Halo region plasma parameters in the West end-cell. . . . . . . 72

3.2 Core region plasma parameters in the West end-cell. . . . . . . 72

4.1 Summary of ECRH absorption efficiencies (all values in \%). . . . 111

4.2 Summary of Fokker-Planck code results. . . . . . . . . . 136 


\section{List of Figures}

1.1 TMX-U features: (a) coil set and (b) magnetic-flux surfaces. . . . 6

1.2 Axial profiles of the TMX-U end-cell magnetic field and theoretical electrostatic potential. . . . . . . . . . . . 7

1.3 ECRF heating systems in the West end-cell. . . . . . . . . 9

1.4 Timing of fueling and heating systems for the typical TMX-U hotelectron discharge. . . . . . . . . . . . . . 11

1.5 Typical results from routine TMX-U diagnostics: (a) line density, (b) diamagnetism, (c) $x$-ray spectrum. . . . . . . . . . . 14

1.6 Sequence of events in the hot-electron plasma formation. . . . . . 18

1.7 Pipe chart of ECRH absorption within the TMX-U end-cell. . . . . 19

1.8 The ECRH power balance. . . . . . . . . . . . . . 21

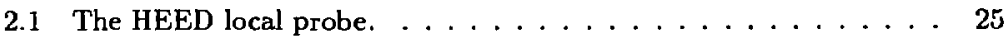

2.2 HEED probe positioning in TMX-U. . . . . . . . . 26

2.3 HEED probe electronics block diagram. . . . . . . . . . 28

2.4 HEED probe circuit schematics: (a) limiter circuit; (b) x-ray detector; (c) thermocouple. . . . . . . . . . . . 29

2.5 The HEED probe data acquisition system. . . . . . . . . 30

2.6 X-Ray detector power sensitivity versus Maxwellian temperature. . 33

$2.7 \mathrm{X}$-ray detector sensitivity data. . . . . . . . . . . 36

2.8 HEED probe calibration setup. . . . . . . . . . 38

2.9 Results of the HEED probe calibration. . . . . . . . . . 39

3.1 HEED probe limiter positions mapped to the West end-cell midplane. 43

3.2 ECRH waveforms for the West end-cell systems on $11 / 14 / 86$; (a) 10-kG heater, (b) 5-kG heater. . . . . . . . . . . 4 45

3.3 Peak diamagnetism plotted for five limiter scans, phi values of (a) $90^{\circ}$, (i) $70^{\circ}$, (c) $60^{\circ}$, (d) $50^{\circ}$, and (e) $0^{\circ}$.

3.4 Limiter scan consistency: (a) unperturbed peak diamagnetism, (b) midplane magnetic-field offset. . . . . . . . . . . . . 48

3.5 Contours of hot-electron energy-density. . . . . . . . . . . . 49

3.6 The azimuthally-averaged total perpendicular-pressure profile in the West end-cell. . . . . . . . . . . . . . . . . . 50

3.7 Curvature-Drift surfaces for various midplane pitch angles: (a) $85^{\circ}$, (b) $75^{\circ}$, (c) $65^{\circ}$, (d) $45^{\circ} \ldots \ldots \ldots \ldots \ldots \ldots$

3.8 Comparison of the diamagnetism scrape-off contours with the $65^{\circ}$ pitch-angle drift orbits. 
3.9 Spatial variation of the PECE temperature; (a-d) HEED probe scans, and (e) RF probe scan. . . . . . . . . . . . . .

3.10 Spatial variation of the 98 and $60 \mathrm{GHz}$ PECE signals; (a-d) HEED probe scans, and (e) RF probe scan.

3.11 The unperturbed 98 and $60 \mathrm{GHz}$ PECE signals as a function of shot-number for the $11 / 14 / 86$ experiment.

3.12 The super-hot component density profile for various azimuths; (a) $90^{\circ}$, (b) $50^{\circ}$, and (c) $0^{\circ} \ldots \ldots \ldots \ldots \ldots \ldots \ldots \ldots$

$3.1398 \mathrm{GHz}$ PECE scrape-off contours. . . . . . . . . . . . 61

3.14 Drift orbit eccentricity versus midplane pitch-angle. . . . . . 62

3.15 Hot-electron pitch angle distribution inferred from the PECE

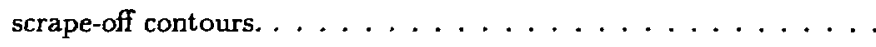

3.16 The azimuthally averaged super-hot component pressure profile in the West end-cell. . . . . . . . . . . . . . 64

3.17 Components of the perpendicular pressure for $11 / 14 / 86$ shot 32 . $\quad 66$

3.18 Line-density data from the limiter-scan experiment: (a) time history-probe retracted; (b) time history-probe fully inserted; (c) Unperturbed average line density versus shot number. . . . . .

3.19 Plasma line-density data for the four HEED probe scans, $\phi$ values of: (a) $90^{\circ}$, (b) $70^{\circ}$, (c) $60^{\circ}$ and (d) $50^{\circ}$.

3.20 Electron end-loss spectra measured on the East fan by the (a) ELA, (b-c) EELS.

3.21 Determination of the plasma boundary by open drift surfaces in the West end-cell. .

3.22 Time-history overlays of (a) diamagnetism, and (b) super-hotcomponent line density.

3.23 Delay time to DML break and PECE line density saturation versus limiter midplane radius.

3.24 Temporal development of the hot-electron boundary.

3.25 Comparison of the plasma expansion data with two simple pressure models.

3.26 Magnetic field profiles: (a) $\mathrm{x}$-axis variation at 5 -kG location; (b) axial variation at $r=0 . \ldots \ldots \ldots \ldots$

3.27 Typical ECRH waveforms for the B-field scan experiment. . . . . . 84

3.28 B-field-scan experiment super-hot-component data: (a) diamagnetism, (b) temperature, (c) line density, (d) column density. . . . 86

3.29 West end-cell plasma structure variation with $\frac{\Delta B}{B_{0}}$. . . . . . . 89

4.1 The first-pass 5-kG ECRH transmission array. . . . . . . . . . 95

4.2 Time history of ECRF absorption in the West end-cell: (a) 5-kG ECRH, (b) 10-kG ECRH, (c) global ECRF absorption.

4.3 ECRH absorption versus limiter midplane radius for four limiter scans. 
4.4 ECRF absorption efficiency versus $\frac{\Delta B}{B_{0}}$ : (a) West global absorption, (b) East global absorption, and (c) East first-pass 5-kG absorption. 100

4.5 Midplane density profiles along the $x$-axis for the MECH power-deposition calculation. . . . . . . . . . . . . 102

4.6 Simulated first-pass absorption of the 5-kG ECRH beam: (a) ray trajectories, (b) power-deposition contours. . . . . . . . . . . . 104

4.7 First-pass 5-kG absorption with purely annular super-hot electrons: (a) ray trajectories, (b) power-deposition contours. . . . . . . . 105

4.8 Results of fundamental ray tracing: (a) ray trajectories, (b) powerdeposition contours. . . . . . . . . . . . . . 107

4.9 Time history of energy storage parameters: (a) diamagnetism, (b) confined power, (c) super-hot line density, (d) super-hot temperature. . . . . . . . . . . . . . . 113

4.10 Imaginary part of the wavenumber versus $\frac{\Delta B}{B_{0}}$ for $28 \mathrm{GHz}$ ECRH. . 114

4.11 Confined power fraction versus $\frac{\Delta B}{B_{0}}$ in the West end-cell. . . . . 116

4.12 Time history of the axjal power loss: (a-b) ECRF heating waveforms, (c) axial power loss. . . . . . . . . . . . . . . 120

4.13 EELS electron energy spectra: (a) $10-20 \mathrm{~ms}$, (b) 20-30 ms, (c) $30-40$ ms. . . . . . . . . . . . . . . . . . . . 121

4.14 Time histories of bolometer array signals: (a) $y=-4.2 \mathrm{~cm}$, (b) $\mathrm{y}=-$

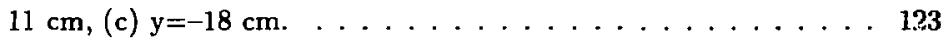

4.15 Variation of axial electron losses with field offset: (a) 10-kG ECRH modulated operation, (b) 5-kG ECRH modulated operation. . . . 124

4.16 Axial loss fraction versus $\frac{\Delta B}{B_{0}}$ in the West end-cell. . . . . . . . 126

4.17 Temporal variation of three radial loss parameters: (a) power, (b) temperature, (c) density. . . . . . . . . . . . . . . . 129

4.18 Radial energy loss versus probe midplane radius. . . . . . . . . 131

4.19 Comparison of the measured radial loss spatial structure with the calculated magnetic-curvature drift orbits. . . . . . . . . . . 132

4.20 Variation of radial loss parameters with $\frac{\Delta B}{B_{0}}$ : (a) temperature, (b) power. . . . . . . . . . . . . . . . 134

4.21 Fokker-Planck calculation of the halo electron distribution function. 138

4.22 Temporal variation of the power-balance terms: (a) absorbed power, (b) confined power, (c) axial-loss power, (d) radial-loss power, (e) missing power. . . . . . . . . . . . . . . . . . . 141

4.23 Variation of the power balance terms with $\frac{\Delta B}{B_{0}}$ : (a) both ECRH systems active, (b) 5-kG ECRH only, (c) 10-kG ECRH only. 


\section{Preface}

This dissertation presents experimental work that is the result of an unusual collaboration between myself and the Lawrence Livermore National Laboratory. Although such university/industry collaborations are not unusual at Stanford, they require particularly special arrangements at LLNL. The collaboration was arranged through the efforts of Drs. Angus Hunt, Thomas Simonen, Fred Coensgen, and my principal advisor, Prof. Oscar Buneman.

This work would not have been successful without the help of many people. Most particularly, I want to thank Dr. Robert Horton. More than anyone else, Robert acted as my research advisor at LLNL, providing technical and moral support throughout my many months of work on TMX-U. Also most helpful in this role was Dr. Barry Stallard. My work was further sustained through the continuing support of my principal dissertation supervisor at LLNL, Dr. Thomas Simonen. I also want to thank Drs. Gary Smith, Bill Nevins, Ron Cohen, Linda LoDestro, Yoshi Matsuda, and Tom Rognlien of the theory group, who frovided assistance in modeling my data. Obtaining the data required many individuals to create and operate my diagnostic systems on TMX-U. In particular, 1 am grateful to Brad Rice, Dan Seligman, Glen Cobb, Eric Conger, Dan Behne, Jeff Moller, Mike Brown, and Bill Meyer.

Finally, I want to thank my wife, Lucille Mitrovich. Lucy supported me physically and emotionally throughout this long and trying time, while tolerating, more or less, my many long hours at work. 


\section{Chapter 1}

\section{Introduction}

Controlled thermonuclear fusion offers mankind a virtually inexhaustible source of energy. A fusion power plant could supply an enormous amount of energy at negligible fuel cost with projected lower levels of radioactivity than comparable fission plants. Magnetic mirrors are a possible containment device for the fusion reaction, and the tandem mirror with thermal barrier ${ }^{1}$ is the most sophisticated recent application of the principles of magnetic-mirror confinement. The tandern mirror experiment-upgrade (TMX-U) at the Lawrence Livermore National Laboratory (LLNL) was the first experiment to investigate the thermal-barrier tandem-mirror concept. One attractive feature of the tandem magnetic mirror as a commercial power reactor is that the fusion reactions occur in an easily accessible center-cell. On the other hand, complicated end-cells are necessary to provide magnetohydrodynamic (MHD) stability and improved particle confinement of the center-cell plasma. In these end-cells, enhanced confinement is achieved with a particular axial potential profile that is formed with electron-cyclotron range-offrequency heating (ECRF heating, ECRH). By modifying the loss rates of electrons at spatially distinct locations within the end-cells, the ECRH can tailor the plasma potential profile in the desired fashion. Specifically, the thermal-barrier concept requires generation of a population of energetic electrons near the midplane of each end-cell. To be effective, the transverse (to the magnetic field) spatial structure of the hol-electron plasma must be fairly uniform. In this dissertation we characterize the spatial structure of the ECRH-generated plasma, and determine how the structure builds up in time. Furthermore, the plasma should efficiently absorb the ECRF power, and a large fraction of the electrons must be well confined near the end-cell midplane. Therefore, we also examine in detail the ECRH power balance, determining how the ECRF power is absorbed by the 
plasma, and the processes through which that power is confined and lost.

The study of ECRH is also relevant to present-day and planned tokamak fusion experiments. The conventional tokamak design employs a transformer coupling to drive the required toroidal current through the plasma. The inductive coupling necessitates a pulsed operation. Tokamak researchers are presently seeking a stcady-state method to produce this current, and one promising alternative involves the use of ECRF heating. To produce a current, the ECRF must heat only those electrons with a particular sense of rotation about the torus. This is achieved by injecting the ECRF at an angle to the magnetic-field lines, so that only electrons with a particular sign of $v_{\|}$satisfy the Doppler-shifted resonance ${ }^{2}$. As the ECRH is absorbed in a spatially localized region, the radial current profile can thereby be optimized. The problems involved in depositing ECRH power at a specific location in the presence of magnetic field gradients are similar in tokomaks and tandem mirrors.

In addition, the diagnostic technique deve'oped in this work to study the radial lass of electrons in TMX-U can be of general use in experimental plasma physics. In particular, the concept could prove useful as a high-speed measurement of the power carried by electrons onto limiter or divertor surfaces in Tokomaks. Such a measurement could provide data on power balance, plasma edge region instabilities, or current-drive efficiency. The technique may also be useful in inertialconfinement fusion studies of $x$-ray production with high-powered lasers, where the power balance in the corona plasma is of interest.

\subsection{The Tandem Mirror Experiment-Upgrade}

A simple magnetic mirror consists of two regions of high magnetic field, $B_{\max }$, on opposite sides of a region of lower field, $B_{0}$. Due to the Lorentz force, $\vec{F}=$ $q \vec{v} \times \vec{B}$, charged particles exhibit helical orbits around the magnetic field lines. Furthermore, the gradient in the magnetic field causes some of the particles to be reflected at a particular feld strength. We define the particle pitch angle by $\theta=\tan ^{-1}\left(v_{\perp} / v_{\|}\right)$where the ratio is to be evaluated at the point of minimum magnetic field, or midplane of the mirror. In the absence of any plasma potential, 
all particles with pitch angles greater than

$$
\theta_{c}=\sin ^{-1}\left(\frac{1}{\sqrt{R}}\right)
$$

are confined within the mirror; $R=B_{\max } / B_{0}$ is the mirror ratio. Particles with smaller pitch angles are lost from the mirror, and therefore $\theta_{c}$ is termed the losscone angle ${ }^{3}$. In TMX-U the mirror ratio is $R \approx 4$, so $\theta_{c} \approx 30^{\circ}$.

Usually, particles are lost from a magnetic mirror by pitch-angle scattering into the loss cone. Unless $T_{e} \gg T_{i}$, the electron collision frequency is much larger than that of the ions, and a mirror-confined plasma acquires a potential

$$
\phi=\frac{T_{e}}{q} \ln \left(\frac{n_{m}}{n_{0}}\right),
$$

where $T_{e}$ and $T_{i}$ are the electron and ion temperatures, respectively, $n_{0}$ the plasma density at the midplane, and $n_{m}$ the density at the peak magnetic fieid. The plasma potential expels ions and confines the electrons in such a fashion as to exactly balance the loss rates of the two species.

A tandem mirror consists of two magnetic mirrors, the end-cells, linked together by the center-cell (see figure 1.1(a)). Except for small perturbations introduced by the transition coil set, the magnetic field in the center-cell is purely solenoidal and axisymmetric. On the ether hand, the end-cell mirrors have a more complicated topology termed minimum- $B$. In such a mirror, the magnetic field has an absolute minimum at the center of the midplane; both radial and axial magnetic wells are present. The quadrupole-symmetric realization of the minimum-B topology used in ' $\mathrm{CMX}-\mathrm{U}$ results in magnetic flux surfaces that are in general elliptical. If the surfaces are defined to be circular at the midplane, then elsewhere they become elliptical with large eccentricity in regions close to the magnetic field maxima. The major axes of the flu: surface ellipses are rotated $90^{\circ}$ on opposite sides of the end-cell midplane; see figure 1.1(b). Plasma confined in a minimum- $\mathrm{B}$ configuration is not subject to $\mathrm{MHD}$ interchange instabilities that vastly lirnit the particle confinernent of simple mirrors ${ }^{4}$. In a tandem mirror, the end-cells provide average magnetohydrodynamic stability to plasma in both the end-cells and center-cell ${ }^{5}$.

In a thermal-barrier tandem mirror, the axial plasma potential profile is adjusted to entance confinement. Specifically, a large, spatially localized positive 
potential is created on the outboard (that is, away from the center-cell) side of the end-cell; this region is referred to as the plug. Also, a potential depression (relative to the center-cell potential) is created in the vicinity of the end-cell midplane (see figure 1.2). The potential depression is called the thernal barrier, because it blocks cold thermal electrons passing from the center-cell. The thermal barrier isolates the plug region from the cold electrons, enabling production of the positi re poiential despite large thermal electron densities in the center-cell ${ }^{1}$.

ECRH is used to create the barrier and plug potentials. Second-harmonic ECR.F is applied near the end-cell midplane to create a population of energetic mirror-trapped electrons. If the majority of electrons confined near the midplane are well confined, the plasma potential in this region decreases as the average electron and ion confinement times become more nearly equal. The magnitude of the potential depression is given by

$$
\phi_{b}=T_{e c} \ln \left(\frac{n_{c}}{n_{t}}\right),
$$

where $n_{c}$ is the center-cell density, $n_{t}$ is the thermal electron density at the end-cell midplane, and $T_{e c}$ is the center-cell electron temperature. Thus, barrier formation requires sufficient ECRF heating at the midplane to keep a large fraction of the end-cell electrons trapped at the midplane ${ }^{6}$. Fundamental ECRF heating is uised to produce the plug potential. By changing the perpendicular velocity of the electrons on the slope of the mirror, close to the loss cone, the ECRH causes a spatially localized reduction in the electron ronfinement. A cold electron is either trapped in the mirror, where it is further heated and trapped by the second-harmonic ECRF, or the electron is "kicked" into the loss cone. Both processes serve to create the positive potential, although the former is more desirable because it efficiently uses the ECRF power.

To create an effective thermal barrier, the mirror-trapped electron population must have certain characteristics. Early theoretical calculations indicated that an end-cell density of $5 \times 10^{12} \mathrm{~cm}^{-3}$ was necessary to allow operation with a center-cell density of $2 \times 10^{13} \mathrm{~cm}^{-3}$. To form an effective barrier, a large fraction, $>80 \%$, of the end-cell electron denrity must be mirror-trapped. The desired temperature for these electrons is $30 \mathrm{keV}$. At this temperature, the electrons are sufficiently well confined to allow creation of the necessary density with a 
reasonable amount of ECRH power ( $220 \mathrm{~kW}$ ). Spatially, we desire the hot-electron density to peak toward the $\mathrm{z}$ axis of the machine, and extend radially to the center-cell limiter, which corresponds to $r \approx 19 \mathrm{~cm}$ in the end-cell. Also, the hot-electron plasma must be azimuthally symmetric, as such asymmetries can drive radial transport of center-cell ions ?. With an effectivs thermal barrier, production of the plug potential should only require efficient absorption of ECRH at the outboard fundamental resonance.

\subsubsection{Physical Description}

TMX-U consists of two minimum-B, quadrupole-symmetric magnetic mirrors, each about $3 \mathrm{~m}$ in length, linked together by an 8-m long center-cell solenoid. At both ends of the center-cell, an additional coil set provides a transition to the quadrupole end-cells. Figure 1.1(a) shows the TMX-U magnet coil set. Physically, TMX-U is divided into seven regions and is arbitrarily oriented East-West. Proceeding from East to West the regions are East end-fan, East end-cell, East transition, center-cell, West transition, West end-cell, and West end-fan. Figure 1.2 shows the on-axis magnetic field profile. Further information about the TMX-U magnetic field profiles can be found in section 3.3.1.

The magnetic field coils are enclosed in a stainless-steel vacuum vessel with a total volume of $2 \times 10^{5}$ liters. Low base pressures (typically $3 \times 10^{-8}$ Torr) are maintained by gas adsorption onto various walls and liners within the vessel. Before each discharge, three to five monolayers of titanium are sublimated onto these surfaces to produce a high gas pumping rate. $540 \mathrm{~m}^{2}$ of these surfaces are cooled to liquid nitrogen temperatures $(77 \mathrm{~K})$ to further improve the vacuum conditions.

For all of the operation described in this dissertation, the plasma was fueled with deuterium from an annular gas-box located near the center-cell midplane. Additionally, a small amount of gas was provided during the first few milliseconds of the discharge by gas nozzles in the transition regions. 


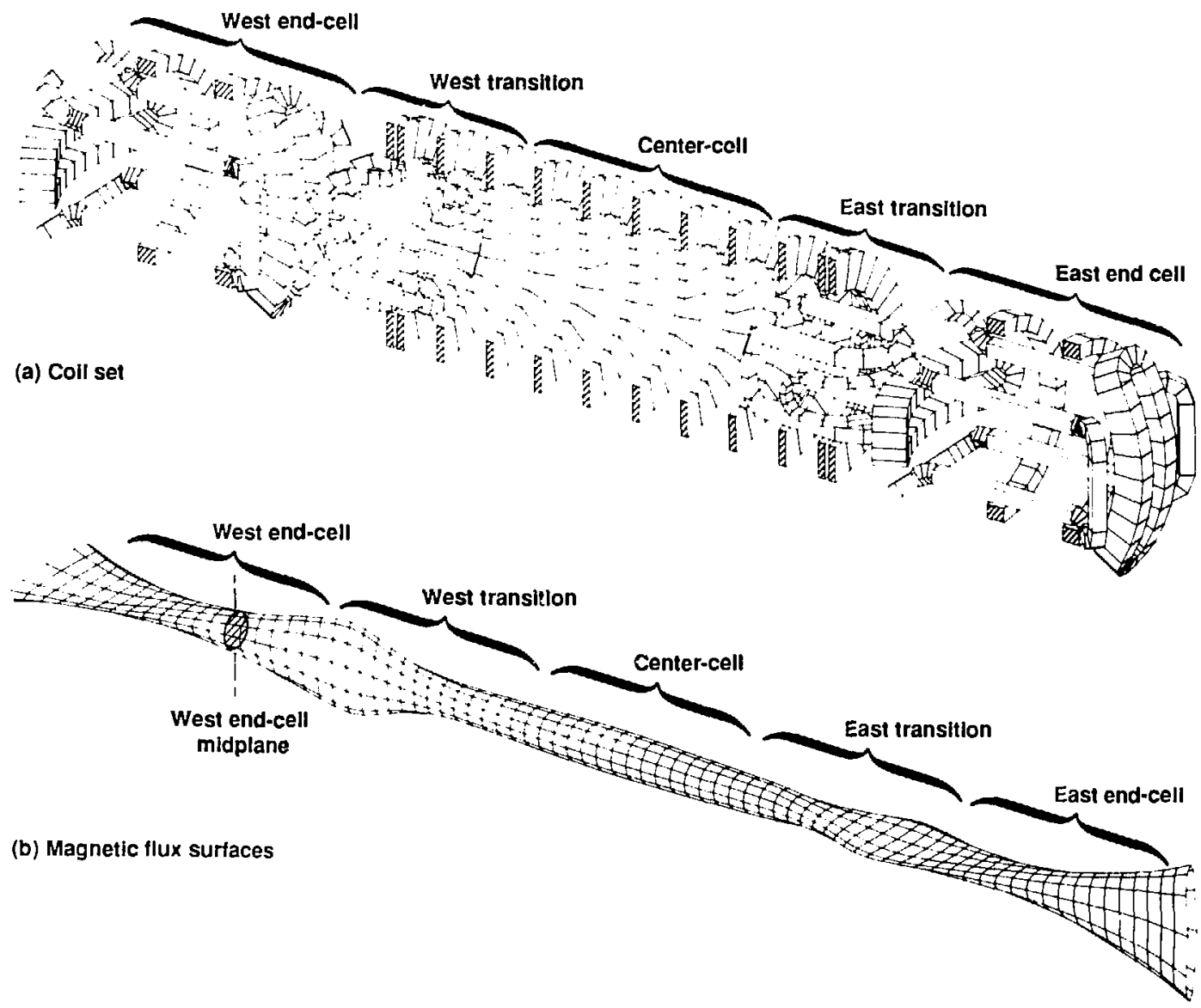

Figure 1.1. TMX-U features: (a) coil set and (b) magnetir-flux surfaces. 


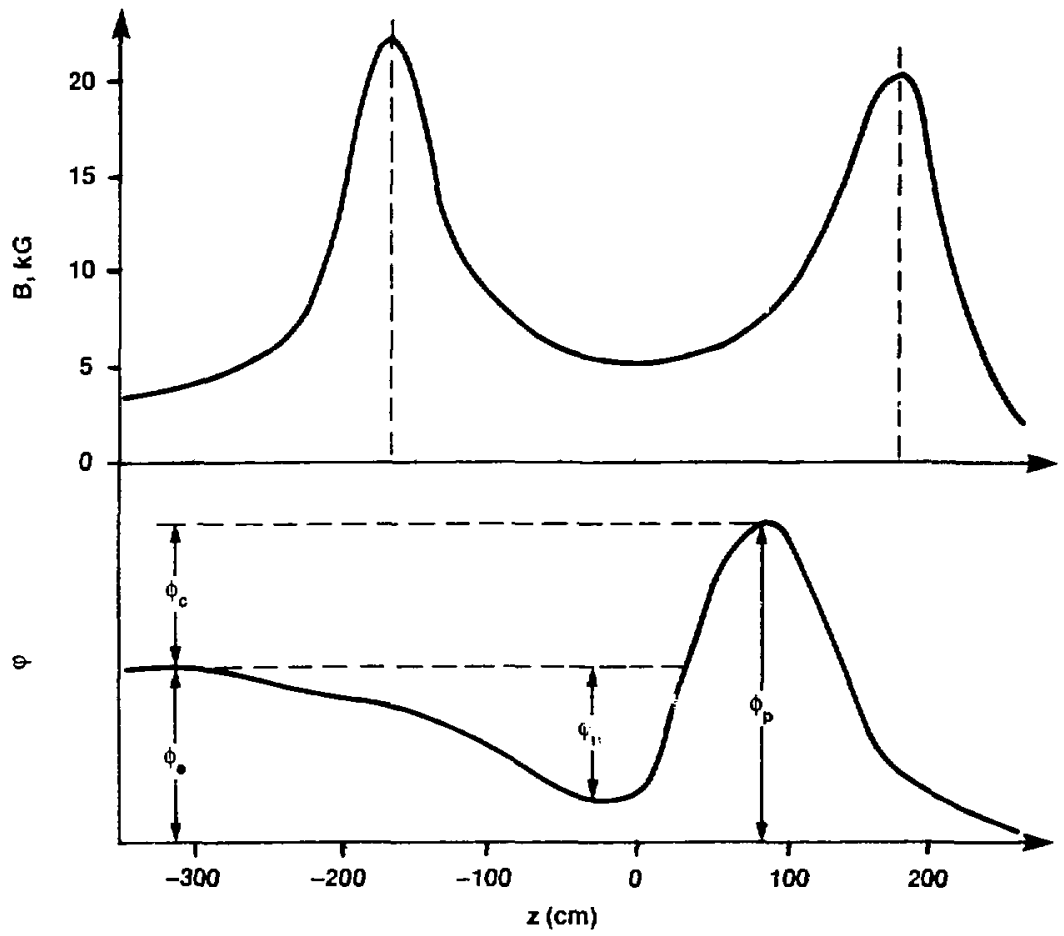

Figure 1.2. Axial profiles of the TMX-U end-cell magnetic field and theoretical electrostatic potential. 


\subsubsection{ECRF Heating System}

In TMX-U, 28-GHz microwave radiation is applied at two locations on the outboard side of the end-cell midplane. At these locations, the magnetic field strength corresponds to the fundamental and second-harmonic of the electron gyro-frequency, i.e., where the wave frequency $\omega_{0}=\omega_{c e} \equiv e B / m$, and $\omega_{0}=2 \omega_{c e}$, respectively. Figure 1.3 shows the physical orientation of the ECRF heating systems in the two end-cells. The fundamental heating is applied at the outer 10-kG magnetic field location; this will be referred to as the $10-\mathrm{kG}$ heater. The launching structure is a grill-like "uniform heater" ${ }^{8}$ providing roughly constant illumination across the long axis of the plasma, which is highly elliptical at these axial locations. The radiation from the uniform heater propagates at $45^{\circ}$ to the magnetic field, and is predominantly (75\%) polarized $\vec{E} \perp \vec{B}$. To create uriform illumination, the 10-kG heater beam pattern had to be made larger than optimal, and a good deal of the 10-kG radiation simply misses the fundamental resonance; as a consequence, the first-pass 10-kG heater absorption efficiency is poor (see section 4.1.5). Second-harmonic heating is applied near the plug midplane at a location where the on-axis magnetic field is $5 \mathrm{kG}$, so this will be referred to as the "5-kG heater". Here, the power is delivered in the form of an approximately circular Gaussian beam formed by a grooved "twist reflector" ${ }^{8}$. The beam has an e-fold radius of about $9 \mathrm{~cm}$, passes through the plasma $14 \mathrm{~cm}$ outboard from the magnetic midplane of the end-cell, and is aimed perpendicular to the $\mathrm{z}$ axis with a small inclination of $10^{\circ}$ with resper. to the $\mathrm{x}$ axis. Polarization is $\vec{E} \perp \vec{B}$. At each location the microwave power is provided by a $28-\mathrm{GHz}$ gyrotron tube.

The ECRH power delivered to each transmitting antenna is measured with directional couplers at the gyrotron output. The output from the couplers is routed to a set of attenuatcrs and monitored by a waveguide diode power detector. In the West end cell, the 10-kG system typically delivers $90 \mathrm{~kW}$ of power, and the 5-kG heater provides $120 \mathrm{~kW}$. 


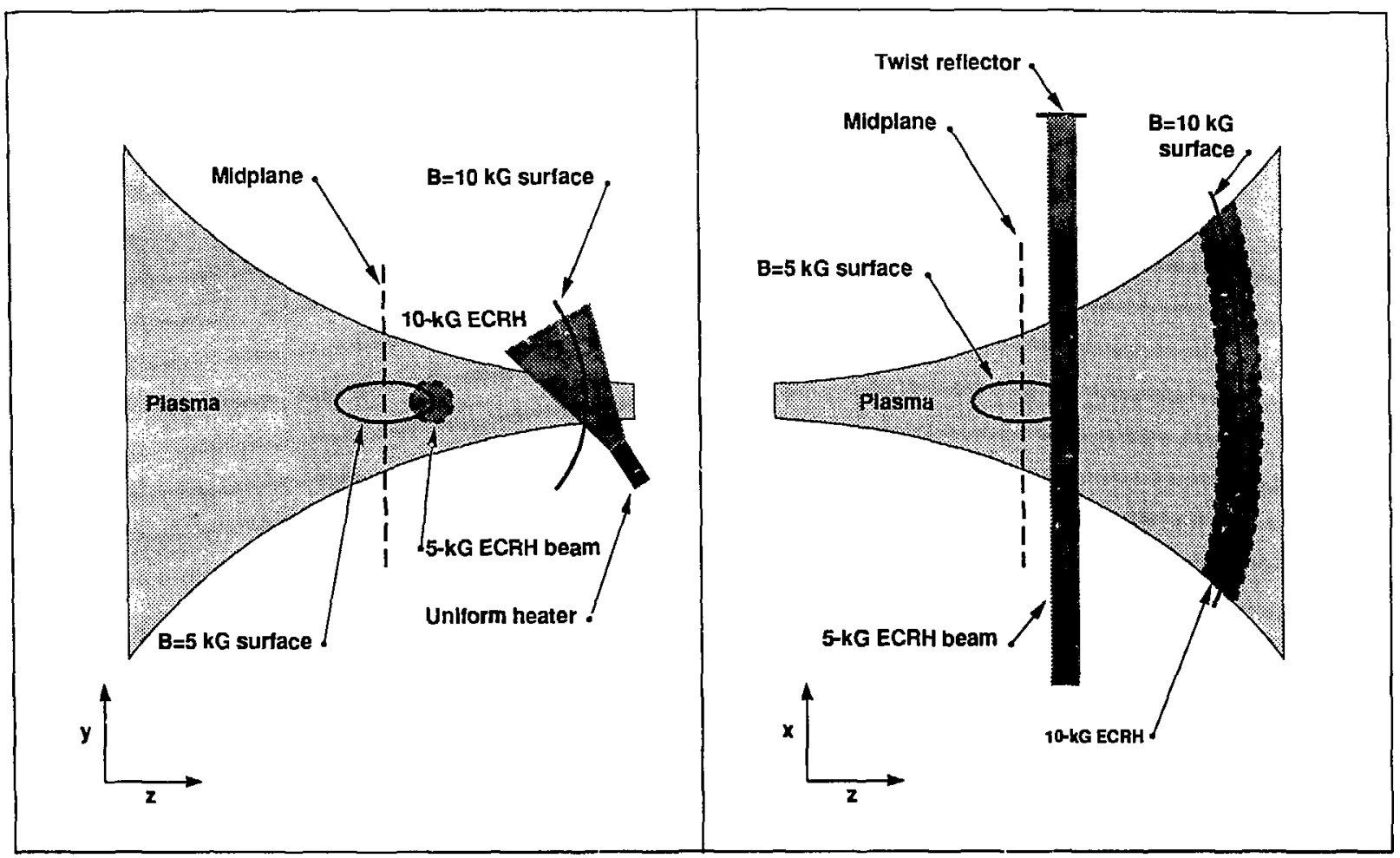

Figure 1.3. ECRF heating systems in the West end-cell. 


\subsection{Features of a Typical TMX-U Discharge}

It is useful to present the time histories of some of the standard diagnostics that monitor all discharges. Several aspects of the TMX-U hot-electron physics are already well known, and shall be summarized here. However, this "standard" picture is superficial; the key details of the physics are the subject of this dissertation.

The timing of the fueling and heating systems is shown in figure 1.4. Gas fueling begins first; the 10-kG ECRH system turns on shortly thereafter, followed by the 5-kG ECRH. For simpliciiy, we concentrated on shots where the other heating systems, neutral bearns and ion-cyclotron range-of-frequency heating (ICKH), were not activated. The 5-kG heater incident power is typically $120 \mathrm{~kW}$, while the $10-\mathrm{kG}$ system power is $90 \mathrm{~kW}$.

\subsubsection{Plasma Line-Density}

Microwave interferometers measure the electron line-density. The instrument detects the phase shift introduced by the plasma in a probe beam of microwave radiation. In the limit that the probe frequency $\omega_{0}$ is much greater than the plasma frequency $\omega_{p e}=: \sqrt{\frac{n_{e} e^{2}}{n_{e} e_{p}}}$, the phase snift is directly proporional to the line density:

$$
\Delta \phi=k_{0} \int\left[\sqrt{1-\frac{\omega_{p}^{\overline{2}}}{\omega^{2}}-1}\right] d l \propto \int n_{e} d l: n_{e l},
$$

where $k_{0}=\omega_{0} / c$ is the vacuum wavenumber for the probe beam, while $n_{e}$ and $n_{e l}$ are the plasma (electron) density and line density, respectively.

In each of the TMX-U end-cells, interferometers are located at three axial locations. One is located near each end-cell midplane, while the other two are about 1 $\mathrm{m}$ inboard and outboard from the midplane. Both midplane interferometers have a vertical viewing angle through the plasma, while the inboard interferometers average through the minor axis of the plasma ellipse. The outbrard interferometers were not fully operational during this work, so we will make use only of the inboard and midplane locations. All of the interferometers were sperated at a frequency of $94 \mathrm{GHz}$, which is approximately ten times the maximum plasma frequency. 


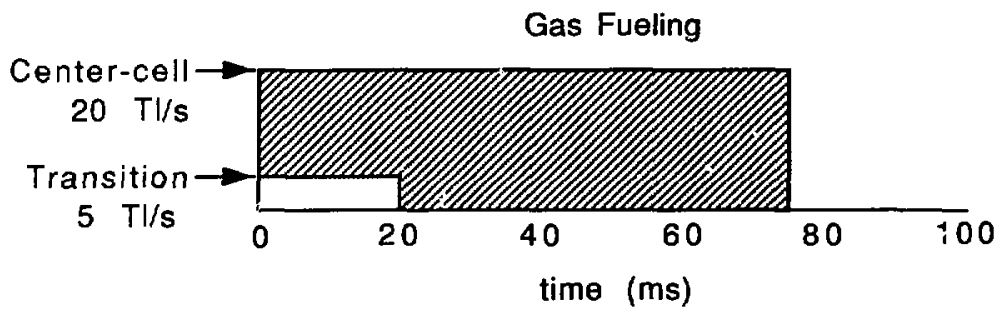

10-kG ECRH

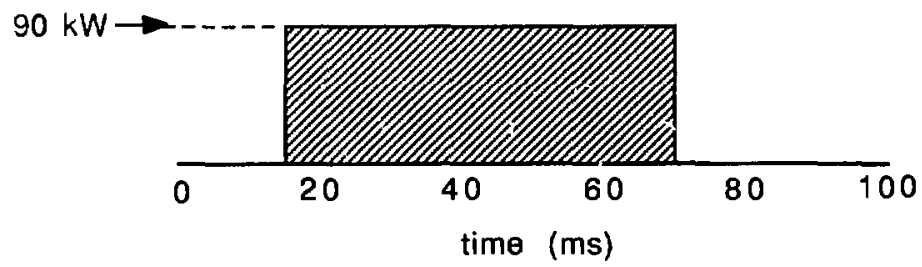

$5 \cdot \mathrm{kG}$ ECRH

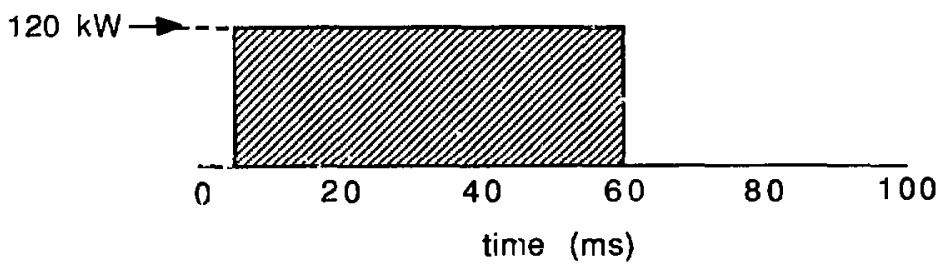

Figure 1.4. Timing of fueling and heating systems for the typical TMX-U hot-electron discharge. 
Figure 1.5 (a) give the line density measured at the midplane of the West endcell. The line density does not build up until the 5-kG ECRH is activated at $t=$ $15 \mathrm{~ms}$; thereafter it builds up to an equilibrium within $5 \mathrm{~ms}$. The effects of the 10 kG ECRH modulation are noticeable during the equilibrium portion of the pulse. When the ECRH is turned off at $t=70 \mathrm{~ms}$, three different exponential decay time components are visible: a fast component, less than $1 \mathrm{~ms}$; an intermediate component, decaying in 5 to $10 \mathrm{~ms}$; and a very slow component. The two slower components correspond to the two decay components of the diamagnetism.

\subsubsection{Diamagnetism}

Plasma diamagnetism is measured directly by placing a loop of wire around the plasma and recording the induced voltage. The integrated voltage is proportional to the plasma magnetic moment per unit length (units of $\mathrm{Acm}$ ), which can be expressed as stored energy per unit length $\left(\mathrm{J} / \mathrm{cm}\right.$ ). (For TMX-U, $10^{5} \mathrm{Acm}=5$ $\mathrm{J} / \mathrm{cm}$.) Also, the loop voltage can be expressed as the change in stored energy per unit length $(\mathrm{kW} / \mathrm{cm}$,

A time history of the diamagnetism is shown in figure 1.5(b). When the 10-kG ECRH system comes on, there is usually little effect; occasionally a small rise of 1-2 $\times 10^{4} \mathrm{Acm}$ occurs in the first 2-3 ms. Thereafter, the diamagnetism does not change until the 5-kG ECRH turns on, whereupon it rises sharpiy for several ms. The derivative of the diamagnetism rapidly reaches a peak, then falls off gradually. When the 5-kG ECRH turns off, the diamagnetisn drops about $10-15 \%$ with a time-constant of 3-5 ms, which is similar to, but generally slightly faster than the intermediate speed decay of the line density. The remaining diamagnetism decays much more slowly, with a time-constant on the order of hundreds of milliseconds.

There are three diamagnetic loops in each end-cell, one locaied near the magnetic midplane, and the other two approximately $1 \mathrm{~m}$ inboard and outboard from the midplane. The subsequent analysis makes direct use of only the midplane diamagnetic loop (DML), because the off-midplane loops produce very small signal levels. However, this indicates that the hot-electron length is less than 2 meters.

A prerious axial scan of $60 \mathrm{GHz}$ perpendicular electron-cyclotron emission (PECE) determined the hot-electron axial profile to be roughly Gaussian with 
a half-length of $60 \mathrm{~cm}^{11}$. (The PECE diagnostic is described in more detail in section 3.1.4.) With this result, the diamagnetism value can be converted to stored energy; peak stored energy is in the range of 1 to $2 \mathrm{~kJ}$.

\subsubsection{X-Ray Emission Spectra}

There are two colinearly aligned $x$-ray spectrometers in both end-cell, each consisting of an $\mathrm{x}$-ray detector connected to a pulse-height analysis system. One system uses a $\mathrm{NaI}$ detector to measure the high-energy (50-1000 keV) $\mathrm{x}$-ray emission, the other uses a high-purity Ge detector to obtain the low-energy (10-50 keV) spectra. Together, they provide measurement of the hot-electron energy spectra in the range of about $10 \mathrm{keV}$ to $1 \mathrm{MeV}$, with a temporal resolution of about 50 $\mathrm{ms}$. In the east end-cell, the viewing angle is $10^{\circ}$ from vertical, while in the west plug it is about $10^{\circ}$ from horizontal ${ }^{12}$.

A typical x-ray energy spectrum is shown is shown in figure 1.5(c). The spectrum is time-averaged over the interval 5-55 ms. It can be fit by the sum of two Maxwellians, with a low-energy or hot component corresponding to a temperature of $20-50 \mathrm{keV}$, and a high-energy or super-hot component with a tempersture of 200-300 keV. These spectra were obtained in the West end-cell. X-ray emission continues long after the ECRH is turned off; the emission is produced by the long-lived super-hot electrons associated with the residual line-density and diamagnetism mentioned previously. Spectra obtained during this period have a non-Maxwellian distribution in which the low-energy component of the distribution is absent.

These $\mathrm{x}$-гаy spectra allow interpretation of the diamagnetism time histories. The small rapid drop in the plasma stored energy when ECRH is discontinued is the disappearance of the hot component, which has an energy-confinement time of 3-5 ms. Plainly, the plasma stored energy is dominated by the superhot component, which has an energy-confinement time on order of hur:ieds of milliseconds. 
(a) Line density

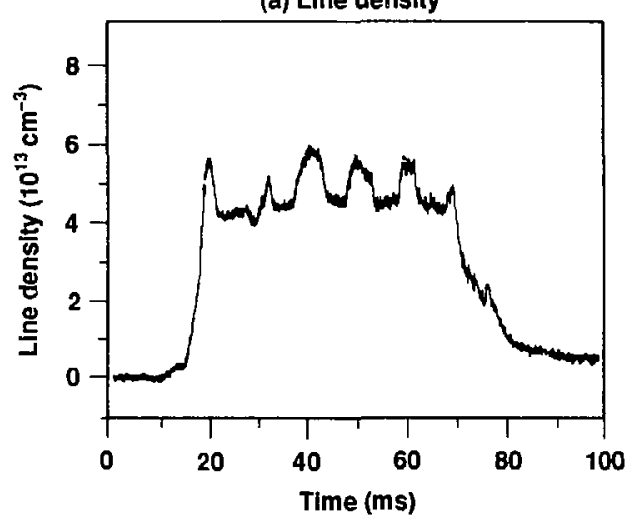

(b) Diamagnetism

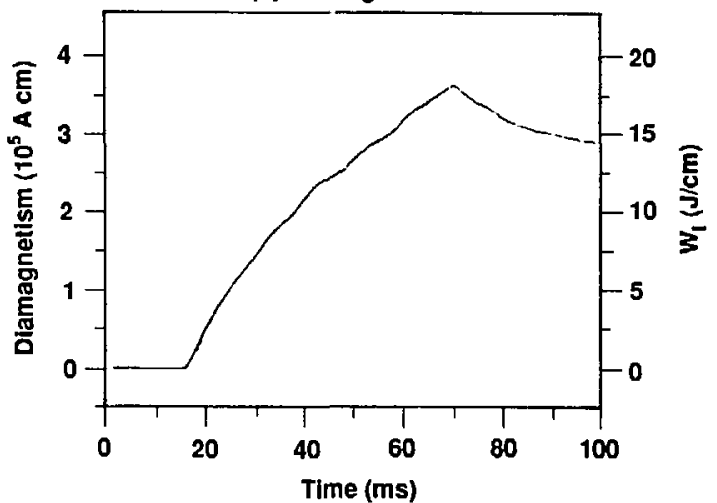

(c) X-ray spectrum

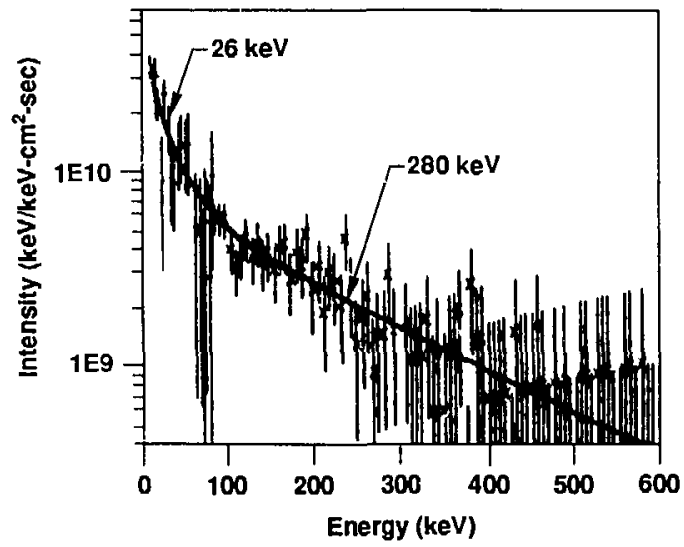

Figure 1.5. Typical results from routine TMX-U diagnostics: (a) line density, (b) diamagnetism, (c) $x$-ray spectrum. 


\subsection{Overview}

This thesis examines the key features of the hot-electron plasma formation and confinement. We study the formation of the plasma, determining the equilibrium plasma spatial structure and its temporal evolution. Prior to this work, the transverse structure of the hot-electron piasma was not known. We also examine the confinement of the hot-electron plasma, determining the mechanisms by which the ECRF energy is absorbed and stored, and the radial and axial channels by which the absorbed energy is lost. Before this thesis work was performed, the ECRH power balance was not understood.

The transverse structure of the plasma is complex. The desired high-density, $30-\mathrm{keV}$ plasma is not formed uniformly across the end-cell midplane. Instead, we find that the plasma consists of two spatially distinct regions: the halc and the core. The halo is an elliptical annulus containing a large density $\left(10^{12} \mathrm{~cm}^{-3}\right)$ of $30-\mathrm{keV}$ hot-component electrons, and a smaller density $\left(3 \times 10^{11} \mathrm{~cm}^{-3}\right)$ of $270-\mathrm{keV}$ super-hot-component electrons. In the West end-cell, the outer boundary has a 31-cm semi-major axis oriented along the y axis, and a 22-crn semi-minor axis; the inner boundary is about 21 by $18 \mathrm{~cm}$ with the same orientation. The halo shape is determined by interaction of magnetic curvature drifts, which dominate the transverse motions of the energetic electrons, with localized absorption of secondharmonic ECRF near the plasma boundary. Within the inner boundary of the halo is the core region. Fewer energetic electrons are found in the core region; there is a small density of super-hot electrons $\left(1.5 \times 10^{11} \mathrm{~cm}^{-3}\right)$, but essentially no hot-component electrons. The core exists on magnetic-field lines that connect to the center-cell gas box, and is only weakly heated by the ECRF. As a result, the core density is dominated by $100-\mathrm{eV}$ cold-component electrons associated with the poorly confined streaming plasma, and $3-\mathrm{keV}$ warm-component electrons.

Throughout this work, we will be making reference to various energy classes of electrons. In general, the observed energy distribution functions are nonMaxwellian. Such deviations from thermodynamic equilibrium are expected in the presence of strong ECRH that produces rapid diffusion in velocity phase space ${ }^{13}$. However, both $\mathrm{x}$-ray and particle end-loss spectra indicate that the distribution can be modeled by the superposition of multiple Maxwellians. Thus, when we 


\begin{tabular}{|c||c|c|c|c|}
\hline \multirow{2}{*}{ Component } & \multirow{2}{*}{$T_{e}(\mathrm{keV})$} & $\tau_{e i}{ }^{a}(\mathrm{~ms})$ & \multicolumn{2}{|c|}{ Density $\left(10^{11} \mathrm{~cm}^{-3}\right)$} \\
\cline { 4 - 5 } & 270 & 610 & 3 & Halo \\
\hline Super-Hot & 30 & 23 & 13 & 0.2 \\
\hline Hot & $30 r e$ \\
\hline Warm & 3 & 0.7 & small & 3.5 \\
\hline Cold & 0.1 & 0.004 & small & 5 \\
\hline
\end{tabular}

Table 1.1. Typical parameters of the energy distribution components.

refer to a "temperature" and "density" of a particular component, we are actually using this model to specify the features of a particular portion of the energy distribution. Table 1.1 summarizes the typical parameters of the various electron energy components.

Plasma formation is dominated by second-harmonic heating near the end-cell midplane. The plasma initially forms along the magnetic axis at the location of the vacuum second-harmonic resonance; refer to figure 1.6. The plasma density and temperature sirnultaneously increase, causing a diamagnetic depression that shifts the second-harmonic resonance to the edge of the plasma. Additional hotelectron plasma is formed at the plasma edge, and, in the shallow radial magnetic well of the TMX-U end-cell, the plasma rapidly grows outward with the plasma pressure profile becoming hollow as the expansion proceeds. After 5-10 ms, the expansion is limited by poor confinement associated with open magnetic-curvature drift surfaces that connect to the plasma boundary. Thereafter, the localized deposition of ECRH power at plasma edge causes the plasma structure to separate into the halo and core regions.

We observe experimentally that fundamental heating generates only warmcomponent electrons, and does not directly create hot or super-hot electrons. When the second-harmonic resonance is not present in the end-cell, no appreciable plasma diamagnetism or hot-electron end-loss is observed. This result disagrees with Fokker-Planck simulations of fundamental ECRF heating ${ }^{24}$.

ECRH power on TMX-U is absorbed largely near the second-harmonic, while fundamental heating is weak; see figure 1.7. Heating at the second-harmonic

a Electron-ion $90^{\circ}$ scattering time calculated for a density of $10^{12} \mathrm{~cm}^{-3}$. 
resonance is dominated by first-pass absorption of the 5-kG ECRH beam, but a significant fraction is provided by cavity heating driven by both 5- and 10-kG heaters. Altogether, roughly $70 \%$ of the incident ECRH power is absorbed at the second-harmonic. Absorption at the fundamental occurs almost entirely on the first pass. Because of compromises in the heater design (see section 1.1.2), first pass absorption is small, and the power absorbed at the fundamental is only about $10 \%$ of the total incident power.

The ECRF energy is stored principally in the super-hot-component electrons. Early in the discharge, these electrons become sufficiently energetic to be essentially collisionless. Furthermore, the imaginary part of the wave vector for the ECRF waves propagating in such relativistic plasma becomes almost indepencient of frequency ${ }^{10}$, so the electrons can absorb energy at all points along iheir bounce trajectory, both from cavity fields and the 5-kG beam. Thus, the super-hot electrons store energy in the form of their ever-increasing temperature. Late in the discharge, the fraction of power absorbed by the super-hot-component drops because of changes in the super-hot electron energy distribution and build up of the hot-component in the halo region. The hot-component eiectrons absorb a fraction of the second-harmonic ECRH power, reducing the power available to super-hot electzons. The hot-component electrons also store energy, but, because of their poorer confinement and smaller volume, their contribution is smali, $10-15 \%$ of the total.

Axial lasses are dominant in the plasma core region. In particular, loss of the warm electrons provides about $45 \%$ of the axial power loss, while the remainder comes from loss of the cold plasma ions and electrons. The energy confinement of the warm electrons agrees well with estimates of classical angle-scattering times. During the first few milliseconds of the hot-electron buildup, the end-loss spectrum shows a large quantity of energetic electron loss with a single temperature of 8 $15 \mathrm{keV}$. Thereafter, the spectrum is dominated by the 3-keV warm electrons, while the loss of hot-component electrons indicates only a very small $\left(2 \times 10^{10}\right.$ $\mathrm{cm}^{-3}$ ) core-region density, corroborating the plasma spatial structure and bcild up models presented earlier.

Radial losses of the hot-component electrons are dominant in the halo region. Electrons are trapped and heated by the 5-kG ECRH beam at the beta-shifted 

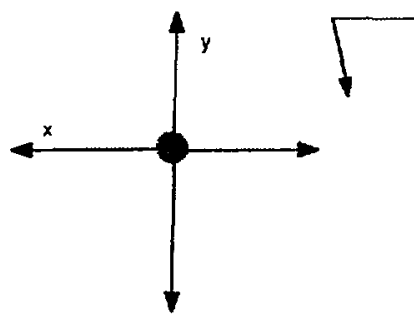

5 KG ECRH Beam

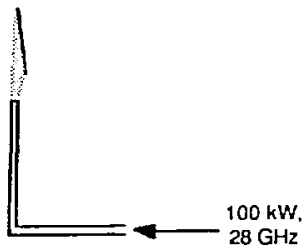

Hot-electron plasma initially forms on z axis.
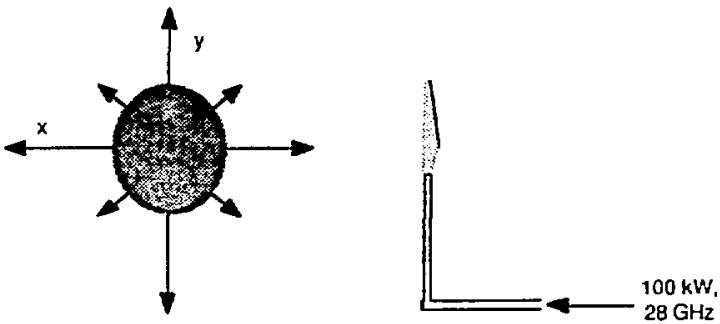

Plasma expands until limited by deteriorating radial confinement.
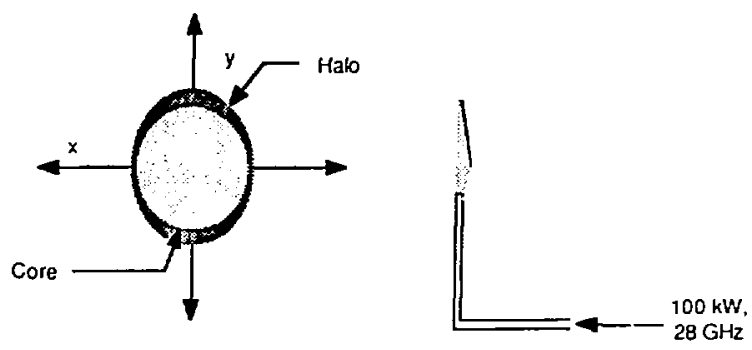

Equilibrium halo/core structure forms.

Figure 1.6. Sequence of events in the hot-electron plasma formation. 


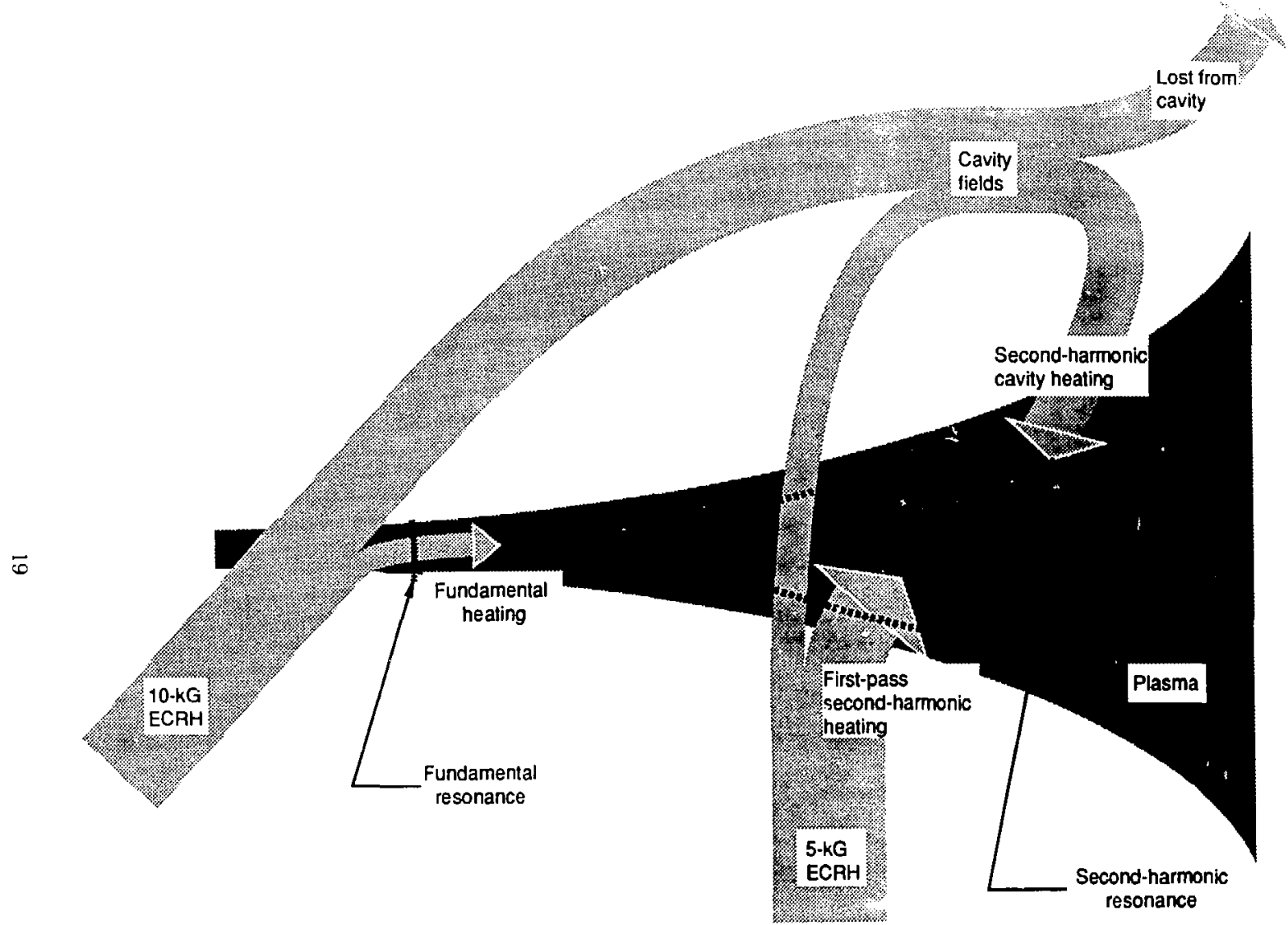

Figure 1.7. Pipe chart of ECRH absorption within the TMX-U end-cell. 
second-harmonic resonance near the plasma boundary. Curvature drift causes the electrons to precess away from the $5-\mathrm{kG}$ beam, and as they drift they rollisionally scatter in pitch angle. Some electrons scatter onto open drift surfaces and are lost; others continue to drift on closed surfaces. Electrons within the separatrix can also be lost by rapid nonresonant neoclassical radial transpert onto the open drift orbits. Measured energy confinement times are approximately 8-12 ms during the ECRH pulse, and 3-5 ms during the decay of the hot-component plasma. The measured energy confinement time in the presence of ECRH is in agreement with calculations that co.nbine Fokker-Planck simulations with estimates of the radial transport rate.

We summarize the ECRH power balance in figure 1.8. During the equilibrium portion of a discharge, $70 \%$ of the incident power is absorbed. The absorbed power divides into three channels. The super-hot electrons absorb $25 \%$ of the incident power, increasing their temperature and total stored energy. Halo electrons absorb $20 \%$ of the power, and are lost radially. The remaining $25 \%$ of the power is absorbed by the plasma core, and is lost axially.

The body of this thesis is divided into four chapters. Chapter 2 describes a novel instrumented limiter probe, designed to evaluate the plasma structure and radial loss mechanisms. In chapter 3 the measurements of plasma structure and formation are presented. Chapter 4 analyzes the absorption and confinement of ECRF power within the plasma, including the roles of the axial and radial energyloss channels. Finally, the conclusions derived from the study are presented in chapter 5. In addition, appendix A presents a description of the two conputer codes that are used extensively in this work, and appendix $B$ summarizes the derivation of the electron radial transport flux. 


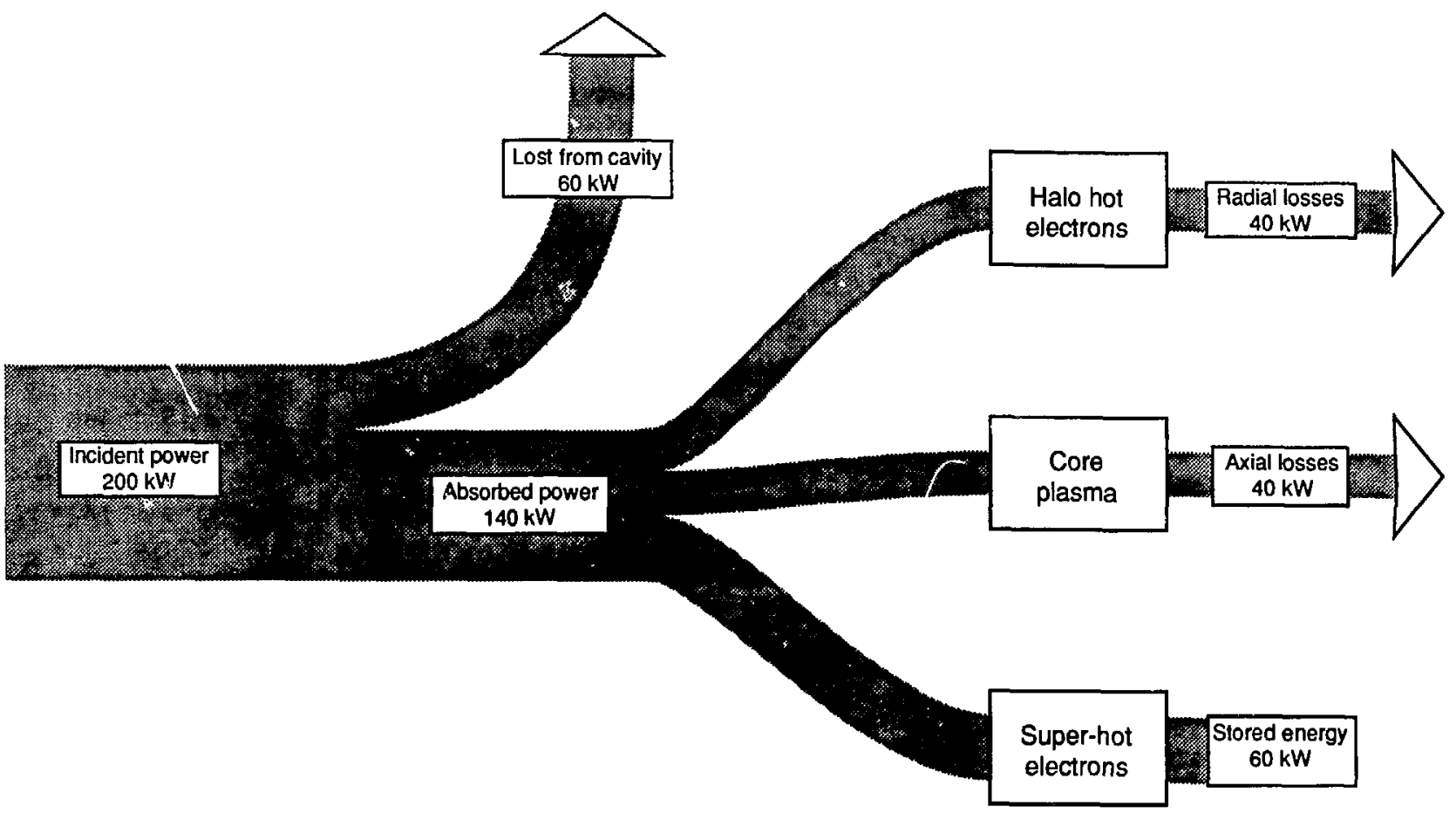

Figure 1.8. The ECRH power balance. 


\section{Chapter 2}

\section{The Hot-Electron}

\section{Energy/Density Probe}

To investigate the TMX-U halo plasma, we created, calibrated, and made use of a novel, yet fairly simple, plasma diagnostic instrument: the Hot-Electron Energy/Density (HEED) probe. This movable probe measures a combination of thick-target $x$-ray emission and electron flux current. The data provide, under the assumption of a Maxwellian energy distribution, a measurement of the total power, temperature, and density of the hot electrons lost along the open drift surfaces in the West end-cell. In addition, the probe serves as hot-electron plasma limiter.

We will describe the instrument in detail. The chapter begins with a physical description of the probe and its configuration in the TMX-U vacuum vessel, followed by a summary of the electronic subsystems of the probe. Next, the theoretical basis for the instrument's operation is presented, and finally the calibration procedures and results are shown.

\subsection{Physical Description}

The HEED probe consists of two major components: a plasma limiter and an $x$ ray detector. To obtain an unshielded view of the $x$-ray emission at certain limiter positions, the $\mathrm{x}$-ray detector must be separate from the limiter. In practice, two detectors were used. The local probe consists of the plasma limiter directly attached to an $\mathrm{x}$-ray detector. The remote probe views the limiter from an alternate position.

The tungsten rod limiter is $8.5 \mathrm{~cm}$ in length and $0.95 \mathrm{~cm}$ in diameter. It is 
supported by an insulating structure to allow collection of the limiter current at a bias voltage of up to $400 \mathrm{~V}$. The rod is tapered to a blunt point at one end. A thermocouple is mounted on the probe $5 \mathrm{~cm}$ from the pointed end.

Each $x$-ray detector, a surface-barrier diode, is enclosed in a lead cylinder with an aluminum jacket. The cylinder has a radius of $3.2 \mathrm{~cm}$ and is $8.9 \mathrm{~cm}$ in length. The shielding thickness is $2 \mathrm{~cm}$ along the walls of the cylinder, and $3.2 \mathrm{~cm}$ on the bottom where the collimator is located. Figure 2.1 shows the local probe. The remote probe has the same shielding structure but none of the limiter hardware. The 2-cm thickness of lead provides at least a factor of 100 attenuation for x-rays up to $400 \mathrm{keV}$ in energy ${ }^{14}$. As the surface-barrier diode sensitivity is down by about a factor of 100 at $100 \mathrm{keV}$, the shielding is sufficient to eliminate background signals due to $x$-rays not entering through the collimator.

The collimating aperture is located in the bottom wall of the lead cylinder. It is angled through the lead shielding to provide line-of-sight to the tip of the tungsten rod. For the local probe the distance from the detector to the tip of the limiter is $4.1 \mathrm{~cm}$, and the aperture is $0.64 \mathrm{~cm}$ in diameter. The remote detector is operated with a much wider aperture to provide sufficient signal strength and field-of-view. The collimator is blocked by a sheet of aluminum $25 \mu \mathrm{m}$ thick. The sheet is transparent to $\mathrm{x}$-rays with energies greater than $6 \mathrm{keV}^{14}$, and blocks particle flux and visible light.

The local probe is attached to a steel boom and inserted into the TMX-U near the top of the west plug midplane at $z=558 \mathrm{~cm}$, as shown in figure 2.2. The bonm enters the machine at an angle $\phi=105^{\circ}$, i.e., $15^{\circ}$ from the vertical. It is motorized to allow remote scanning from $r=50 \mathrm{~cm}$ to $r=20 \mathrm{~cm}$. In addition, the noom can be manually rotated. Since the limiter is offset from the boom attachment (see figure 2.1), this rotation maps out a 12 -cm circle. An arrangement consisting of a stainless steel bellows and Wilson-seal joint allow these translational and rotational motions while maintaining a good vacuum seal.

The remote probe is attached to a different boom, and can be inserted at two different locations. In the first location, the probe enters the machine from the top of the west plug midplane at $z=558 \mathrm{~cm}$, but at an angle $\phi=75^{\circ}$ (see figure 2.2) Here, the remote probe is about $25 \mathrm{~cm}$ from the limiter. The other location is on the south side of the west plug, at an angle of $10^{\circ}$. When inserted here, the 
remote probe is about $90 \mathrm{~cm}$ from the limiter.

\subsection{HEED $P_{\text {robe Electronics }}$}

Three signals are acquired from the HEED Probe. The limiter circuit holds the tungsten rod at a fixed potential while measuring the current. Similarly, the $x$-ray detector circuit must provide bias to the diode and measure the signal current. The thermocouple circuit measures a small, slowly varying voltage to provide a time history of temperature. All signals must be digitized and stored in a computer for later processing. Figure 2.3 shows a block diagram of the HEED Probe electronics.

A schematic of the limiter circuit is shown in figure 2.4. The bias supply is located approximately 5 meters from the probe. The supply provides 10 amperes at up to $400 \mathrm{~V}$. When the probe is operated at zero bias, the supply is replaced by a short circuit. The current is converted to a voltage by a simple resistive shunt on the "low" side of the supply, and is then filtered to prevent aliasing. Due to the large dynamic range of the limiter current signal, two parallel channels of data are E-quired. One channel is amplified to provide good resolution when the current is small. The signals are digitized by the CAMAC data-logger. The sampling rate is adjustable from 1 to $40 \mathrm{kHz}$, and 8 kilobytes of data are stored from each channel with 10 -bit resolution.

The $\mathrm{x}$-ray detector circuit is shown in figure 2.4(b). A CAMAC power supply module provides a compt ter controllable bias voltage. This bias is applied to one side of the diode, and the supply ground acts as common. The signal current flows through the diode into the CAMAC data-logger. This module has a modified front-end that converts the current to a voltage, which is then digitized. The sampling rate and memory size are the same as in the limiter circuit described above. The filter in the current loop prevents aliasing and removes high-frequency noise. The thermocouple circuit simply measures the voltage produced by the thermocouple mounted on the HEED probe limiter [see figure $2.4(\mathrm{c})$ ? . The voltage, which varies from about 1 to $50 \mathrm{mV}$, is amplified by a factor of 50 . 'The resulting signal can then be digitjzed. Due to the very slow time scale of the temperature variations of the limiter, a separate data-logger is used. The sampling rate of $100 \mathrm{~Hz}$ with a record size of 8 kilobytes provides a good record of the limiter 


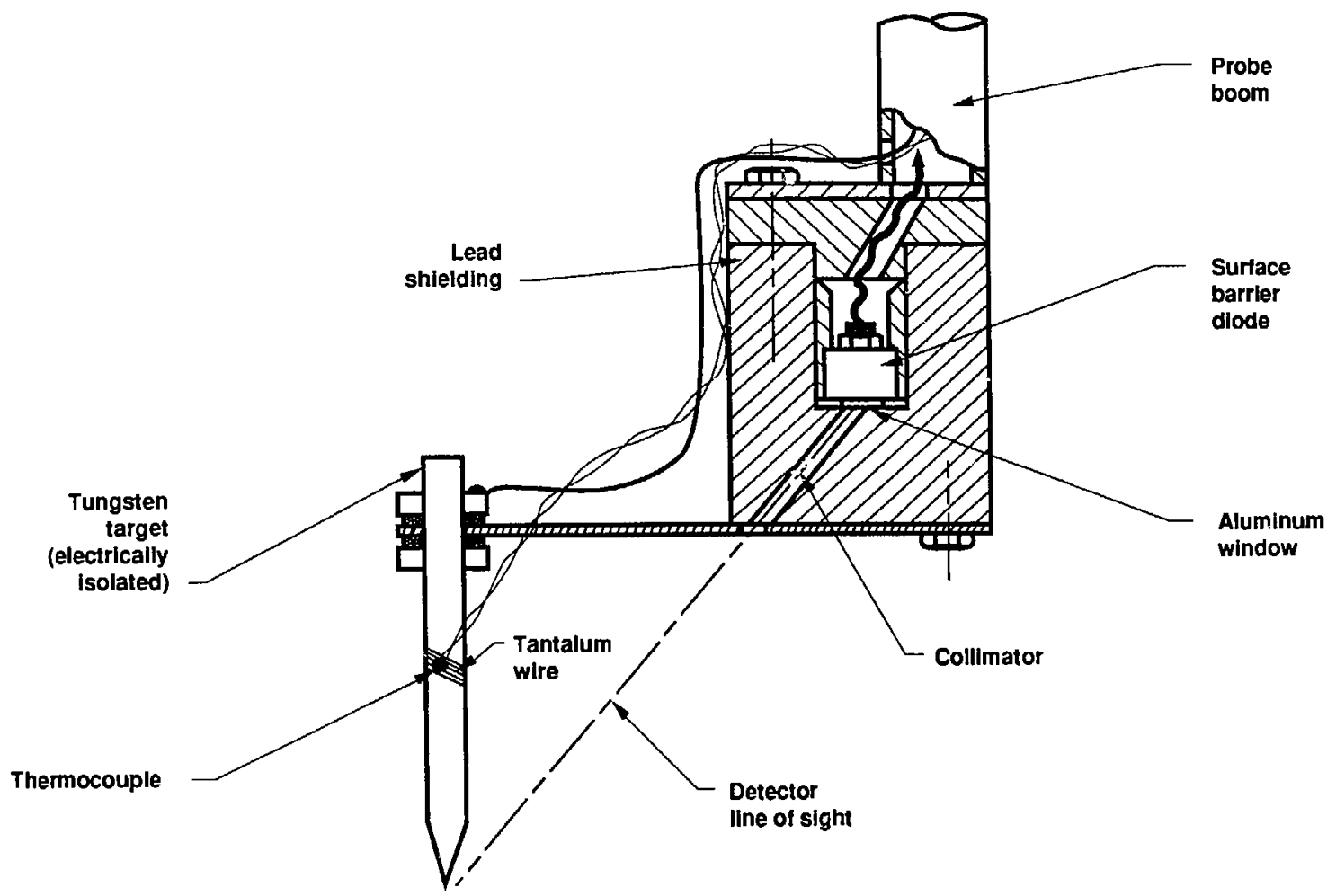

Figure 2.1. The HEED local probe. 


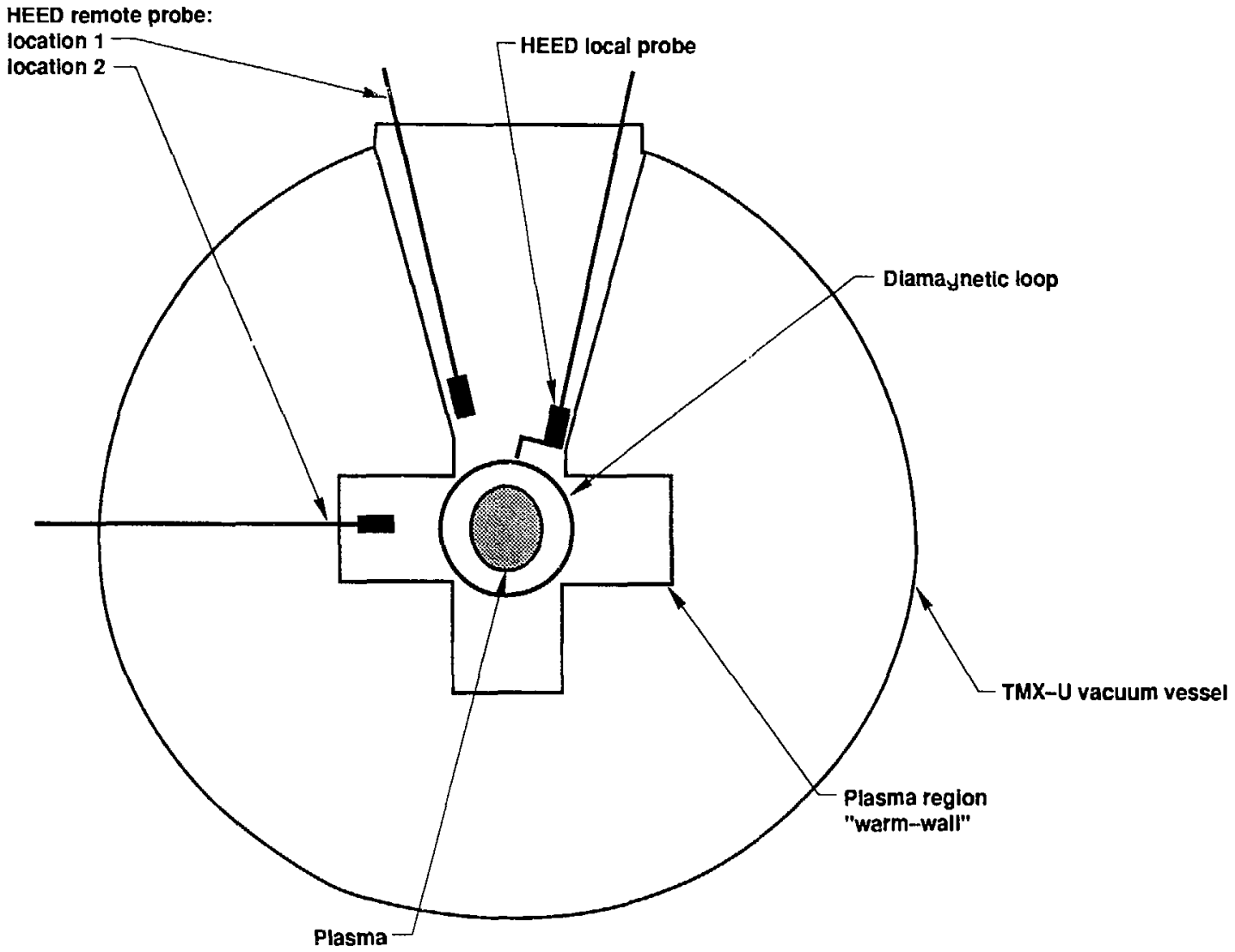

Figure 2.2. HEED probe positioning in TMX-U. 
temperature time history.

The HEED probe digitizes all analog signals using CAMAC recorders adjacent to the TMX-U vacuum vessel. The digital signals are encoded optically and transmitted 40 meters to the data acquisition area through fiber optic cables; this method provides excellent noise immunity. In the data acquisition area the optical signals are decoded and acquired by an HP-320 computer. The computer processes the raw data and provides graphical output. Figure 2.5 shows a block diagram of the HEED probe data acquisition system.

\subsection{Theory of Operation}

The operation of the HEED probe is based upon measurement of two different moments of the hot-electron distribution function, which is assumed Maxwellian. This assumption is analytically convenient, but the principal measurement of the incident electron power is actually relatively insensitive to the particular choice of electron energy distribution, for it is based on the ratio of two similar moments of that distribution. The limiter current gives the electron flux when the plasma distribution is sufficiently energetic. The $x$-ray detector current gives a more complicated moment of the distribution function. However, for a fairly broad range of electron temperatures, the detector current proves to be directly proportional to the total incident electron power. Simultaneous measurement of these two moments determines the power and Maxwellian temperature and density of the hot electrons incident upon the HEED probe limiter.

Assume that a drift, motion of the energetic electrons carries them onto the limiter. If the drift velocity, $v_{d}$, carries electrons of density $n_{e}$ and energy distribution $f(w)$ onto the limiter, then the differential electron flux is

$$
\frac{\partial \mathcal{F}}{\partial w}=n_{e} A_{s} v_{d} f(w),
$$

where $A_{s}$ is the scrape-off area, the cross-sectional area of mirror confined plasma that is drifting onto the probe, and can be estimated as the product of the electron gyro-radius with the hot-electron length. Assuming that the distribution is Maxwellian and that the drifts are dominated by magnetic curvature, the flux can 


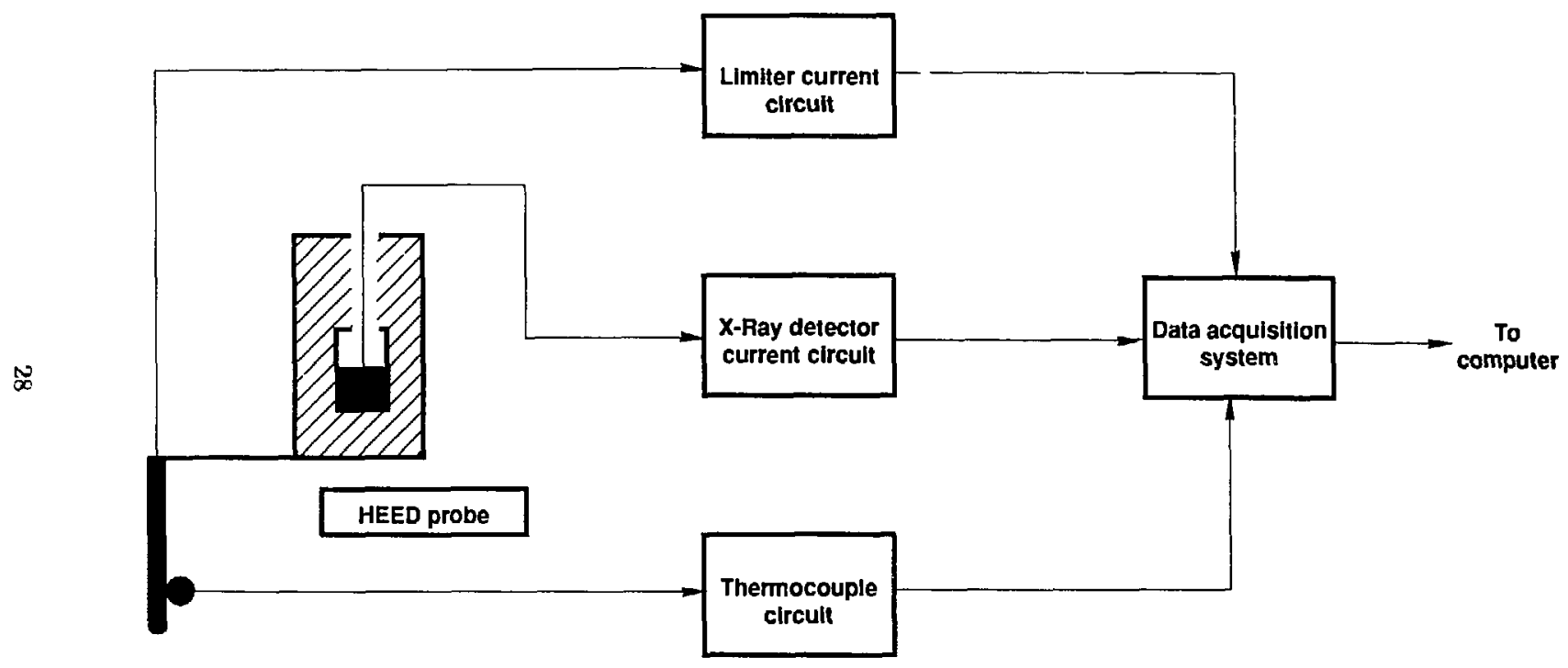

Figure 2.3. HEED probe electronics block diagram. 

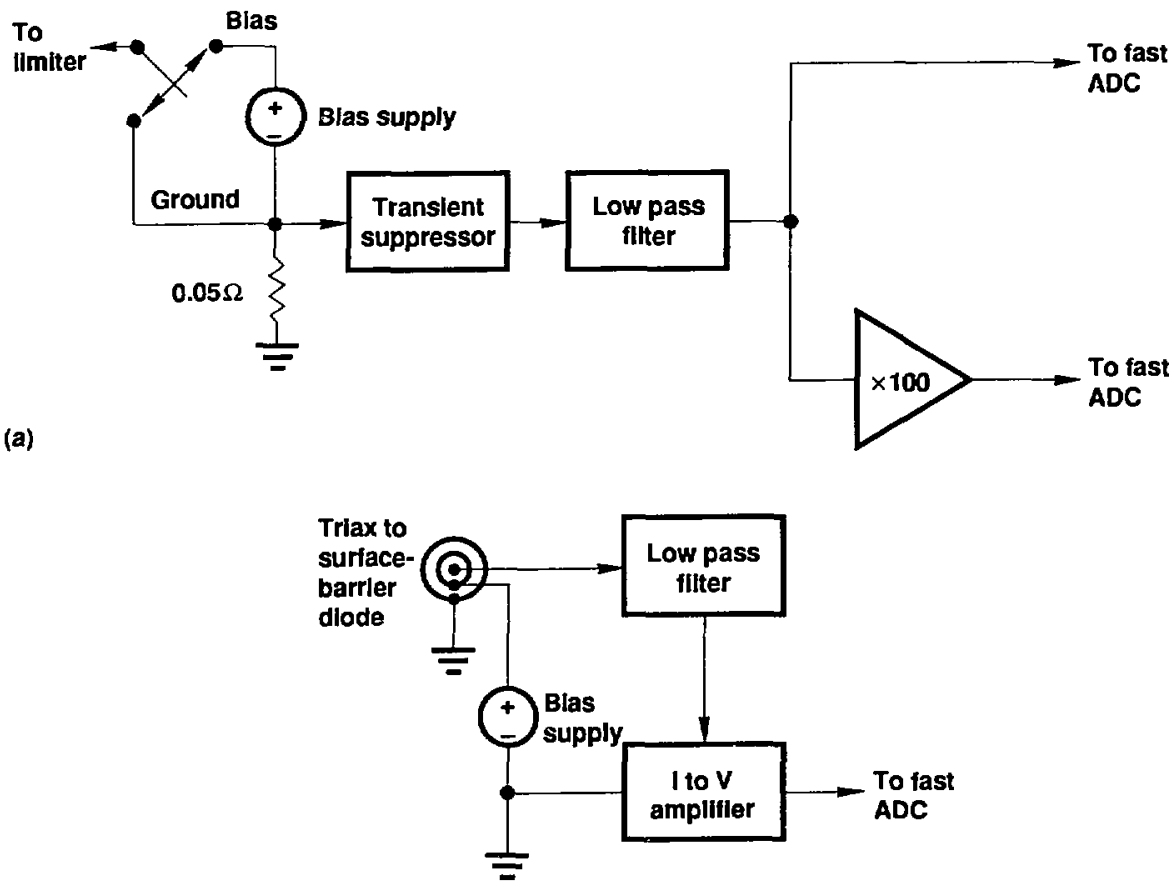

(b)

To

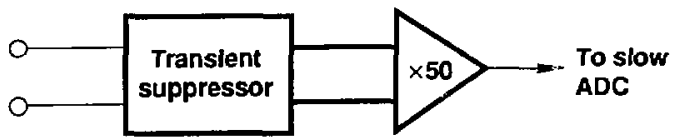

(c)

Figure 2.4: HEED probe circuit schematics: (a) limiter circuit; (b) x-ray detector; (c) thermocouple. 


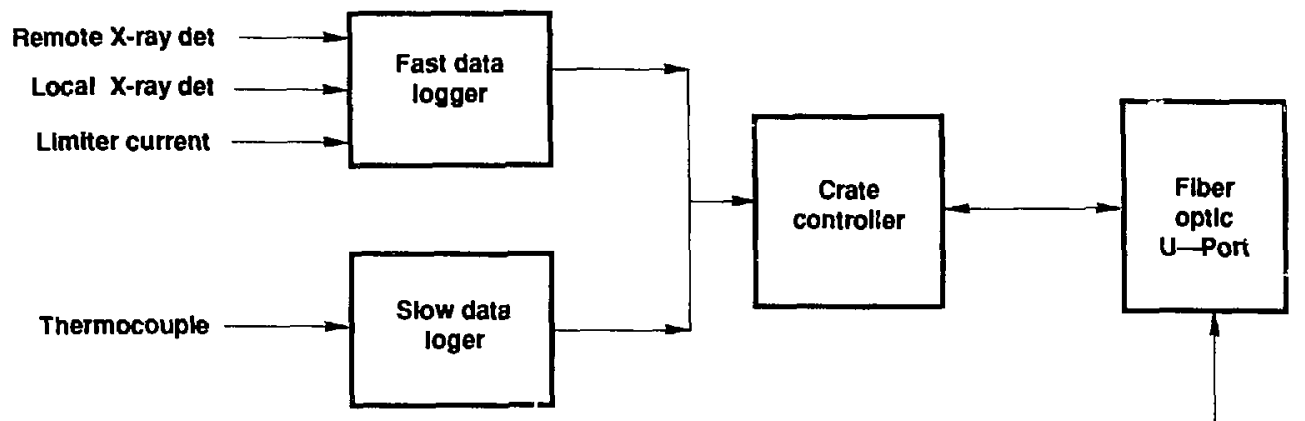

Flber optlc link

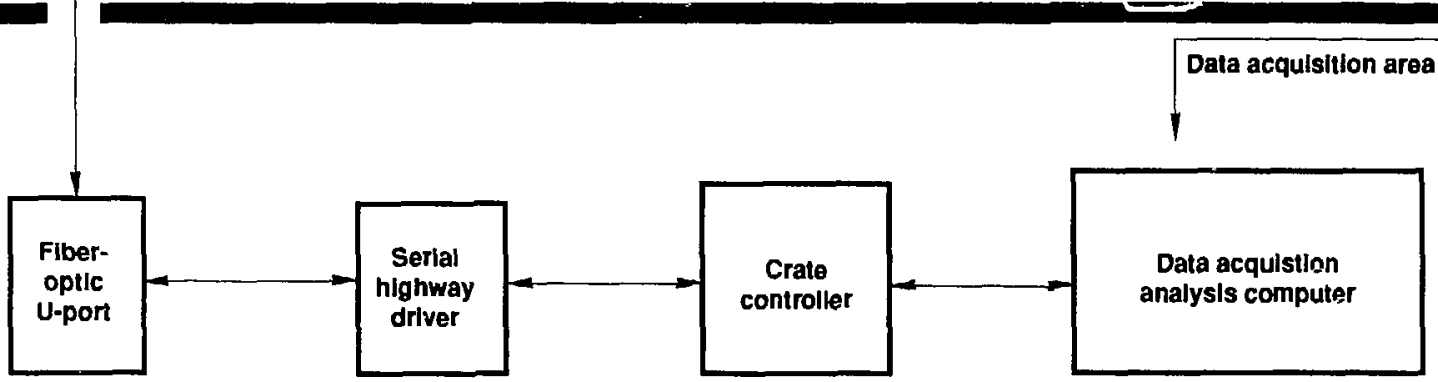

Figure 2.5. 'The HEED probe data acquisition systern. 
be integrated to obtain the total flux, and thereby the limiter current:

$$
I_{p}=e \mathcal{F}=\frac{3 e A,}{2 m \Omega_{0} R_{\mathrm{eff}}} n_{e} T_{e},
$$

where $\Omega_{0}$ is the midplane electron-cyclotron frequency, and $R_{\text {eff }}$ is an effective radius of curvature of the magnetic field.

In a similar fashion, the total electron power lost onto the limiter can be calculated as:

$$
P_{0}=\int_{0}^{\infty} d w w \frac{\partial \mathcal{F}}{\partial w}=\frac{15 e A_{e}}{4 m \Omega_{0} R_{e f f}} n_{e} T_{e}^{2} .
$$

Comparison of eq. 2.3 with eq. 2.2 gives the useful relation:

$$
T_{c}=0.4 \frac{P_{0}}{I_{p}} .
$$

When the electrons impact the probe, they produce $x$-rays with a probability that varies linearly with their energy ${ }^{15}$. The $x$-ray photon flux at photon energy $E$ is obtained from:

$$
\frac{\partial P_{z}}{\partial E}=C Z \int_{E}^{\infty} d w w(w-E) \frac{\partial \mathcal{F}}{\partial w} .
$$

Again for a Maxwellian distribution, the result is

$$
\frac{\partial P_{x}}{\partial E}=C Z P_{0} \frac{\Gamma\left(\frac{7}{2}, x\right)-x \Gamma\left(\frac{5}{2}, x\right)}{\Gamma\left(\frac{7}{2}\right)},
$$

where $x=\frac{E}{T_{e}}$, and $\Gamma(\bullet)$ denotes the incomplete and complete Gamma functions, as appropriate.

The surface barrier diode $\mathrm{x}$-ray detector converts the incident $\mathrm{x}$-ray photons into electron-hole pairs. Low-energy photons are stopped completely within the detector's active region; their energy is converted to current with an efficiency $S_{0}$ that is about $28 \%$. This is true for $\mathrm{x}$-ray energies up to a value $E_{t}$, determined by the $\mathrm{x}$-ray range in silicon. Beyond this energy, the $\mathrm{x}$-rays deposit only a fraction of their energy in the detector, and the detector sensitivity falls off as some power of the photon energy, $\alpha$, which is about 3 . When the detector aperture is blocked by a thin foil, a low energy cutoff in the sensitivity is also introduced. The cutoff is determined by the range of the photons in the appropriate foil metal. The lowenergy cutoff is much sharper than the high-energy cut-off. Thus, the detector 
sensitivity function can be fairly well approximated by the following piecewise function,

$$
S(E)= \begin{cases}0, & \text { if } E<E_{f} \\ S_{0}, & \text { if } E_{f}<E<E_{t} \\ S_{0}\left(\frac{E_{t}}{E}\right)^{\alpha}, & \text { if } E>E_{t}\end{cases}
$$

This description of the detector sensitivity is based only on photoionization. For $x$-ray energies above $100 \mathrm{keV}$, Compton effects become important. Absorption in the detector by Compton scattering can be taken into account by adding a fourth "piece" to the above description, but only the low-energy theory is considered here. The contributions of Compton scattering to the detector sensitivity are negligible for electron temperatures below $200 \mathrm{keV}$.

To obtain the $\mathrm{x}$-ray detector current, the product of the thick-target photon emission spectrum and the detector sensitivity is integrated over photon energy:

$$
I_{d}=\int_{0}^{\infty} d E \frac{\partial P_{z}}{\partial E} S(E)
$$

When the gamma functions are integrated by parts, the result contains 8 terms, each involving yet another gamma function. For brevity, it is not printed here. The $x$-ray detector current is proportional to the electron power multiplied by a function of electron temperature. A useful form, then, is the detector power sensitivity, $S_{x}$, defined as the ratio of detector current to electron power. This is plotted in figure 2.6 for the HEED remote probe using the values of $E_{f}$ and $E_{t}$ obtained below in the section on calibration. The features are quite reasonable. For temperatures less than $E_{f}$, the detector sensitivity is small, then rises quickly for $T_{e}>E_{f}$. As the temperature becomes greater then 2 or 3 times $E_{t}$, the detector power sensitivity approaches a limit

$$
S_{z} \approx C Z S_{0}\left(\frac{\alpha}{\alpha-1} E_{t}-E_{f}\right)
$$

Therefore, for a broad range of tcmperatures, the $\mathbf{x}$-ray detector current is directly proportional to the incident electron power. In this limit, eq. 2.4 then immediately gives the electron temperature from the satio of the $x$-ray detector current to the limiter current:

$$
T_{e} \approx \frac{0.4}{S_{x}} \frac{J_{d}}{J_{p}} .
$$

and the density can be calculated from either of eq. 2.3 or 2.2 . 


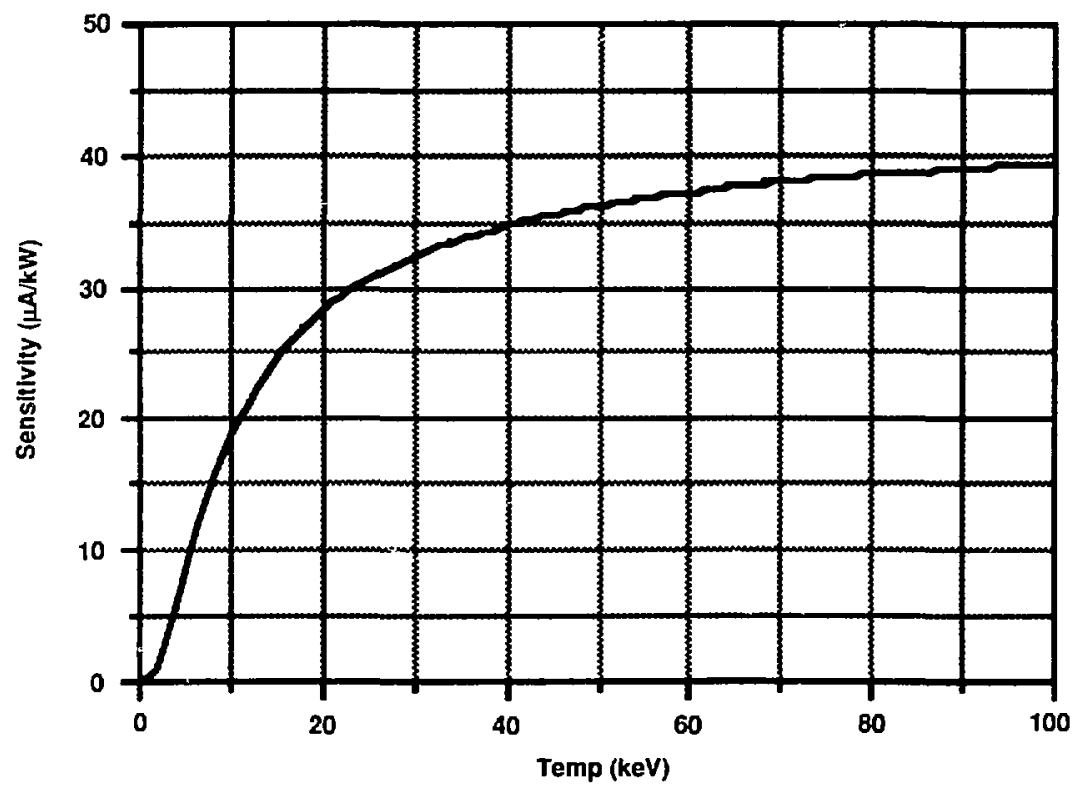

Figure 2.6. X-Ray detector power sensitivity versus Maxwellian temperature. 


\subsection{Calorimetry}

The voltage from a thermocouple mounted on the HEED probe limiter provides a time history of the local temperature. To reasonable accuracy, the temperature rise at the thermocouple, $\Delta T$, is directly proportional to the energy deposited onto the limiter by the electron drift losses, $\Delta U$. The one-dimensional heat equation provides the constant of proportionality:

$$
\frac{\Delta U}{\Delta T}=\sqrt{\frac{\pi e}{2}} C M_{0},
$$

where $C$ is the heat capacity of tungsten, $0.134 \mathrm{~J} / \mathrm{g} / \mathrm{K}$, and $M_{0}$ is the mass of the tungsten between the thermocouple and the tip of the rod.

The relationship between the peak temperature and deposited energy has been modeled in detail using the TOPAZ two-dimensional finite-element heat-transport code ${ }^{16}$. The results provide minor corrections for the probe shape, thermal contact resistance of the thermocouple, and radiative losses from the hot probe tip. When all of these corrections are applied, the result deviates by less than $3 \%$ from the simple one-dimensional model. A somewhat larger correction (up to 6\%) arises from the different spatial distributions of electron loss onto the limiter that occur as the probe is moved. These can be approximately compensated by determining the time delay to the peak temperature at the thermocouple. The overall accuracy of the calorimeter is estimated as $\pm 2 \%$.

The calorimetry provides an absolute calibration of the total power loss. This is used to determine the viewfactor of the remote detector, which views the limiter at varicus angles through an oversized lead collimator. The viewfactor has been estimated using optical techniques, ard this result agrees well with the calrorimetry when the remote detector views the limiter at small angles with respect to the collimator. At other angles the systematic deviation with increasing angle can be as large as a factor of two. Thus, the absolute calibration of the incident energy provided by the calorimetry is generally an essential part of the data analysis. 


\subsection{Calibration}

The HEED probc was calibrated in two ways. First, the $x$-ray detector sensitivity was measured directly as a function of photon energy. Second, the entire probe was calibrated as a system using an electron beam. The measureme:sts show good agreement with the theory outlined above.

The $\mathrm{x}$-ray detector sensitivity was tested in the range 8 to $98 \mathrm{keV}$. Ten data points in this range were obtained by irradiating the detector with the line emission from various radioisotopes. (These data were provided by the Lawrence Livermore National Laboratory L-division X-Ray 'Test group.) The results are shown in figure 2.7, which also shows the results of an $x$-ray Monte-Carlo code that includes all relevant $x$-ray cross sections for photoionization, Compton scattering, and pairproduction. The agreement is very good. For energies in excess of $25 \mathrm{keV}$, the decay is fit quite precisely by $E^{3}$. The cutoff energy, where the sensitivity falls to $1-e^{-1}$, is about $17 \mathrm{keV}$.

The entire probe was calibrated as a system in as realistic a fashion as possibie. An 1-mA electron beam was generated in a side-lab vacuum ves: ' using conventional techniques. The vacuum vessel was cylindrical, approximater. ' $\mathrm{m}$ in length, and $1 \mathrm{~m}$ in diameter. 6-mm lead shielding was placed inside the vessel to attenuate $x$-rays produced by the electron beam. The beam was magnetically focuscd onto the probe limiter, and beam voltage, limiter current, and $x$-ray detector current were measured simultaneously. Figure 2.8 shows the calibration setup. Measureruents were made with the beam energy in the range 5 to $47 \mathrm{keV}$. Tn collect secondary electrons, the limiter was bjased to $+500 \mathrm{~V}$. The $\mathrm{x}$-ray detector was biased to $-300 \mathrm{~V}$, which provides maximum sensitivity.

To interpret the results, the previous theoretical treatment was repeated for a delta-function clectron distribution. Then, values were assigned to the parameters of the piccewise detector sensitivity function. Since this is an approximation to a smooth function, the values for these parameters are not perfectly defined. The $x$-ray detector sensitivity calibration data above gives the decay slope plainly as $\alpha-3$, and the high-energy cutoff value is about $E_{\mathfrak{t}}-17 \mathrm{keV}$. For the lowcnergy cut-off, the 5.3-keV photon energy corresponding to the electron range equal to the thickness of the aluminum window is used ${ }^{14}$. Furthermore, the overall 


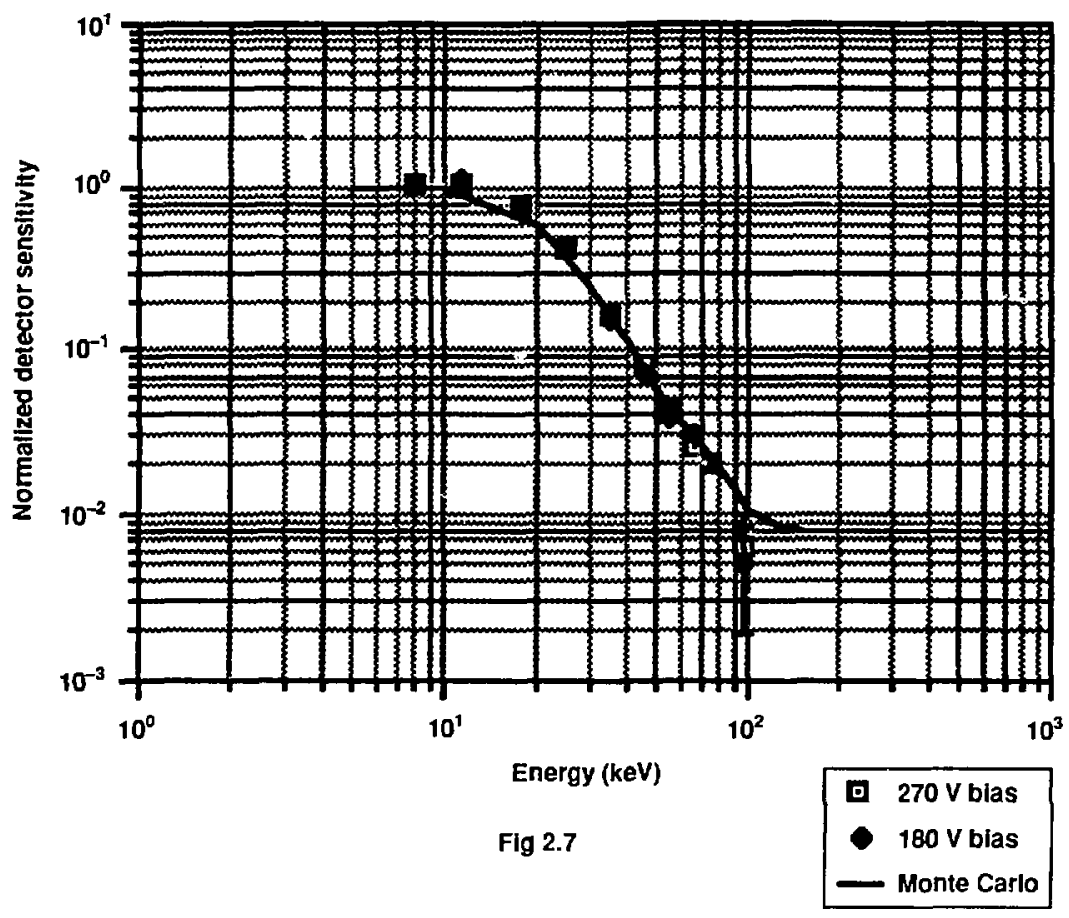

Figure 2.7. X-ray detector sensitivity data. 


\begin{tabular}{|c|c|c|c|}
\hline Parameter & $\begin{array}{c}\text { First Guess } \\
\text { Value }\end{array}$ & $\begin{array}{l}\text { Best Fit } \\
\text { Value }\end{array}$ & $\begin{array}{c}\text { Deviation } \\
(\%)\end{array}$ \\
\hline $\begin{array}{l}\text { Low-Energy } \\
\text { Cutoff, } E_{f}\end{array}$ & $5.4 \mathrm{keV}$ & $6.0 \mathrm{keV}$ & +11 \\
\hline $\begin{array}{l}\text { High-Energy } \\
\text { Cutoff, } E_{t}\end{array}$ & $17.0 \mathrm{keV}$ & $16.5 \mathrm{keV}$ & -3 \\
\hline $\begin{array}{c}\text { X-Ray Production } \\
\text { Constant, } C\end{array}$ & $2.9 \times 10^{-6} / \mathrm{keV}$ & $3.0 \times 10^{-6} / \mathrm{keV}$ & +3 \\
\hline $\begin{array}{l}\text { High-Energy } \\
\text { Slope, } \alpha\end{array}$ & 3.0 & 3.0 & 0 \\
\hline
\end{tabular}

Table 2.1. Summary of HEED probe calibration parameters

multiplicative constant, $C$, is given in the literature ${ }^{15}$ as approximately $2.9 \times$ $10^{6} / \mathrm{keV}$. Using these values as a "first guess," we determined the parameters that gave the best fit to the data based upon the RMS error. Since the result was not particularly sensitive to $\alpha$, it was fixed at the original value of 3 . The results for the remaining three parameters are summarized in table 2.1. For each parameter, the table shows the "first guess" value, the "best fit" value, and the percent deviation. The theory and calibration results are plotted in figure 2.9 . The agreement, both for the parameters and the data, is quite good.

Thus, the calibration bears out the theoretical predictions of the probe performance. The slightly modified parameters for the piecewise detector sensitivity are used to calculate the HEED-probe sensitivity to a Maxwellian distribution of electrons, as was shown in figure 2.6. The calibrated probe provides a measurement the power, temperature, and density of the electrons drifting onto the tungsten limiter. 


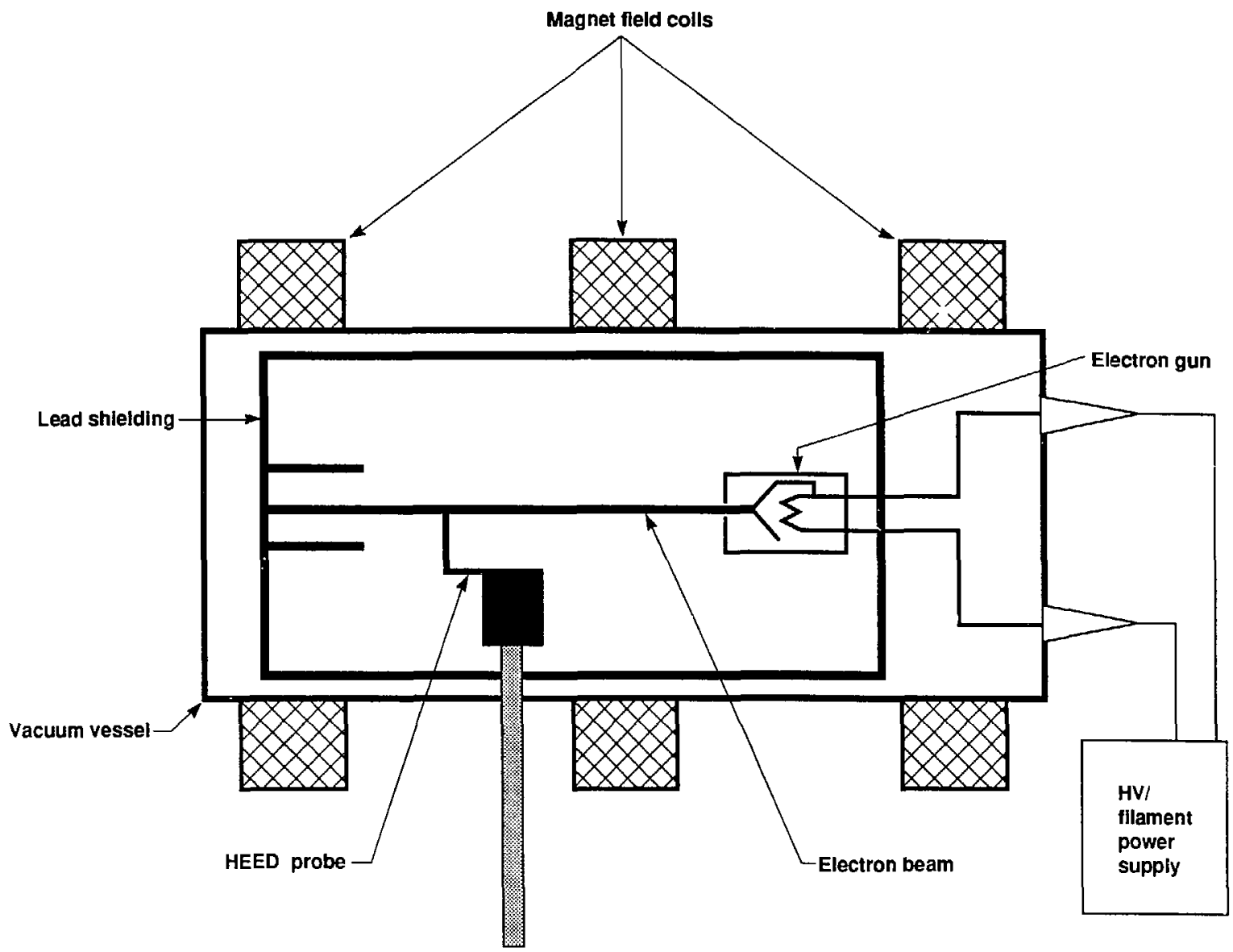

Figure 2.8. HEED probe calibration setup. 


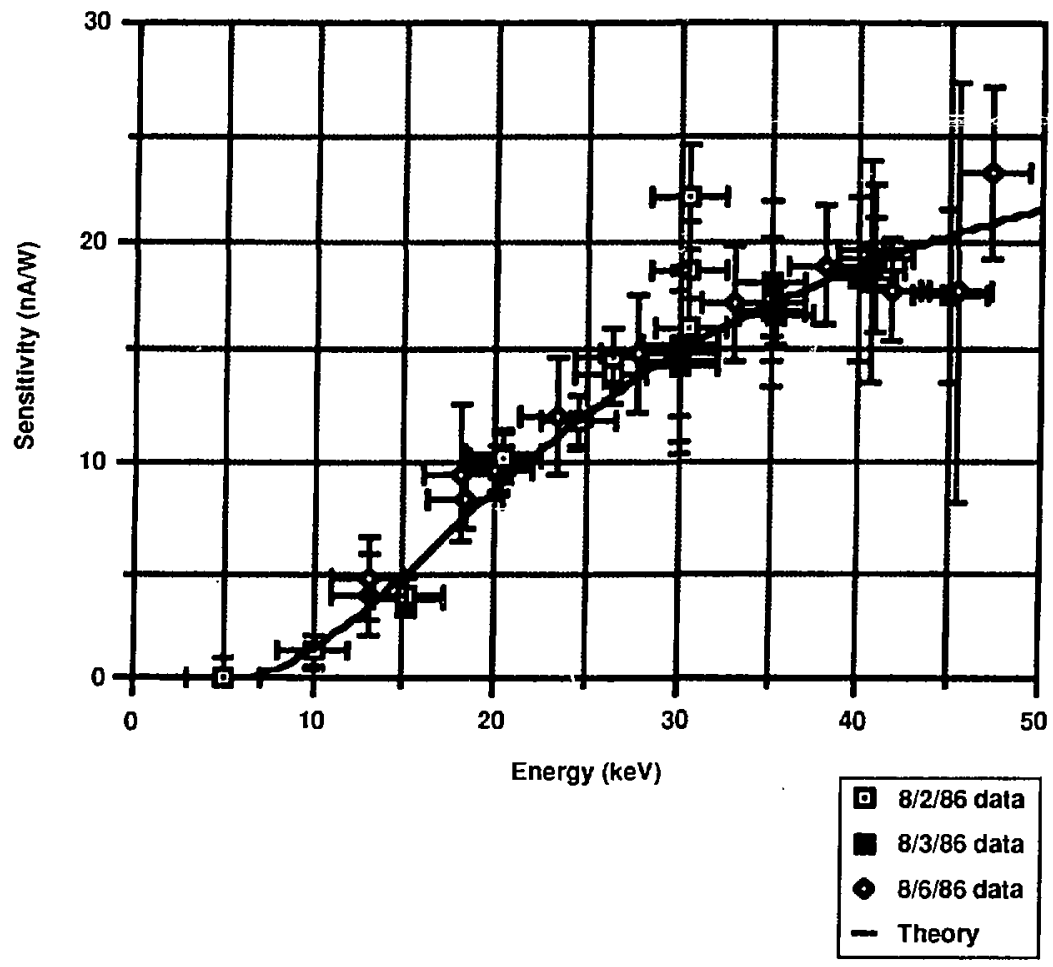

Figure 2.9. Results of the HEED probe calibration. 


\section{Chapter 3}

\section{Hot-Electron Plasma Formation}

In this chapter, we describe the hot-electron plasma formation. First, we consider the equilibrium spatial structure of the plasma, including both the total plasma pressure and the contributions of the various components of the energy distribution. Second, we examine the temporal evolution of the hot-electron plasma boundary. The data for both of these studies come from several limiter probe scans, which will be discussed at length. Finally, we use the results of a vacuum magnetic-field scan to examine the effects of the initial second-harmonic resonance location on the hot-electron plasma formation.

\subsection{Equilibrium Plasma Spatial Structure}

A limiter-probe study in the West end-cell divides the plasma into two distinct regions, a halo and a core. The halo is characterized directly in the limiter probe scan. This region has an elliptical boundary and contains the majority of the plasma pressure, so that the azimuthally averaged pressure profile is hollow. The halo density is dominated by the hot-component electrons associated with the spatially localized ECRH at the second-harmonic. Within the halo is the core region. The average characteristics of the core are inferred from the limiter probe scans, and further information is provided by end-loss measurements. The core density is dominated by cold-component (100 eV) electrons, and mirror-trapped warm-component electrons with a temperature in the range $2-5 \mathrm{keV}$.

We first describe the limiter probe scan experiments that provide the data on the equilibrium spatial structure. Next, we examine the peak values of diamagnetism and late-time (at ECRH turn-of) perpendicular electron-cyclotron cmission (PECE) signals. The variation of diamagnetism with limiter radius provides a 
measurement of the total plasma pressure profile. Then the additional assumption of a multicomponent Maxwellian energy distribution, as discussed in chapter 1, is used to subdivide the total perpendicular pressure into the super-hot $\left(T_{e} \approx\right.$ $200-300 \mathrm{keV})$ and hot $\left(T_{e} \approx 20-50 \mathrm{keV}\right)$ components. The variation of the PECE signals with limiter radius gives information about the spatial profiles of the superhot-component plasma temperature and density. The difference between the two provides an indication of the spatial structure of the hot-component plasma. As the analysis proceeds, we compare the results with the magnetic-curvature-drift orbi:s predicted by the SPLINE code. Finally, the plasma line density measurements are analyzed and compared to the hot-electron measurements. The results indicate that the halo region is dominated by the hot and super-hot electrons. Further, the core plasma is subdivided into two components: a cold, streaming plasma with a temperature of $100-200 \mathrm{eV}$, and a mirror-trapped component. Examination of electron end-loss spectra shows that the mirror-trapped electrons in the plasma core are dominated by the warm-component electrons, with a temperature of $2-5 \mathrm{keV}$.

The complete analysis of the ECRH-generated plasma is carried out for the West end-cell. The East end-cell halo is expected to have a different structure due to the different relativ orientation of the 5-kG ECRH beam and the magneticcurvature-drift surfaces. However, we expect similar core regions in both end-cells.

\subsubsection{Limiter Probe Scans}

We assume that the only sffect of a limiter probe is to limit the plasma boundary to a point tangent to the probe. Two arguments justify this assumption. First, energetic electrons are tightly constrained by the magnetic field; their dynamics should be largely unaffected by the potential variations present in the sheath surrounding material objects inserted into the plasma. These "sifif" electrons should be effectively scraped off as their slow drift motions carry their guiding centers within a gyro-radius of the probe. Second, the probe is not an effective source of additional plasma. Since the probe surface is heated by the incident hot-electron plasma to extremely high temperatures during each discharge, it is cleaned of gas molecules deposited on its surface. However, some amount of the 
metallic probe material is probably evaporated during the discharge, but this high-Z material does not effectively penetrate the plasma due to the very low thermal velocity of the ions. Instead, it is rapidly ionized at the surface of the plasma and lost from the plasma volume. This effect was demonstrated in a previous experiment where Krypton gas was injected during a deuterium discharge in an attempt to enhance $\mathrm{x}$-ray emission for diagnostic purposes. In fact, the $\mathrm{x}$ ray production did not increase until after the plasma line density had decayed. Further evidence comes directly from the variation of the line density during the probe scans: the line density actually decreases as the probe is pushed in to smaller radii, see Section 3.2.5.

On $11 / 14 / 86$, four radial scans of the HEED probe were performed in the West end-cell of TMX-U. The probe was inserted $16 \mathrm{~cm}$ inboard from the West end-cell midplane, and was scanned radially from about $\mathrm{r}=20$ to $40 \mathrm{~cm}$. As the limiter is mounted offset from the probe boom axis, different regions of the plasma can be mapped out by rotating the boom. (More detailed information about the HEED probe is provided in chapter 2.) Figure 3.1 shows the probe scan trajectories flux mapped to the end-cell midplane, where each measurement location is indicated with a symbol. Although these trajectories are not exactly radial, we will identify the scans by their average azimuthal coordinates, that is $\phi=90^{\circ}, 70^{\circ}, 60^{\circ}$, and $50^{\circ}$. In each scan, measurements were taken at five points spaced 2.5 or $5 \mathrm{~cm}$ apart, and, generally, two shots were taken at each location to guarantee a complete data set. The limiter scans were mechanically constrained to midplane radii greater than about $20 \mathrm{~cm}$. Thus, the scans probed the region of the plasma that we term the halo, with midplane radii greater than $20 \mathrm{~cm}$. Detailed information about the plasma interior or core, with midplane radii less than $20 \mathrm{~cm}$, is not provided by this experiment, but average values of the core parameters can be calculated. During the scans the variations of total line density, diamagnetism, PECE line density and temperature, ECRF absorption, and radial and axial power loss were recorded.

Plasma fueling and heating conditions were held constant throughout this experiment. Deuterium gas flow $(22 \mathrm{Tl} / \mathrm{s})$ was adjusted 10 provide an unperturbed West end-cell line density of $5 \times 10^{13} \mathrm{~cm}^{-2}$. In the plasma structure experiment, the $10-\mathrm{kG}$ radiator provided about $90 \mathrm{~kW}$, while the $5-\mathrm{kG}$ operated at $110 \mathrm{~kW}$. 


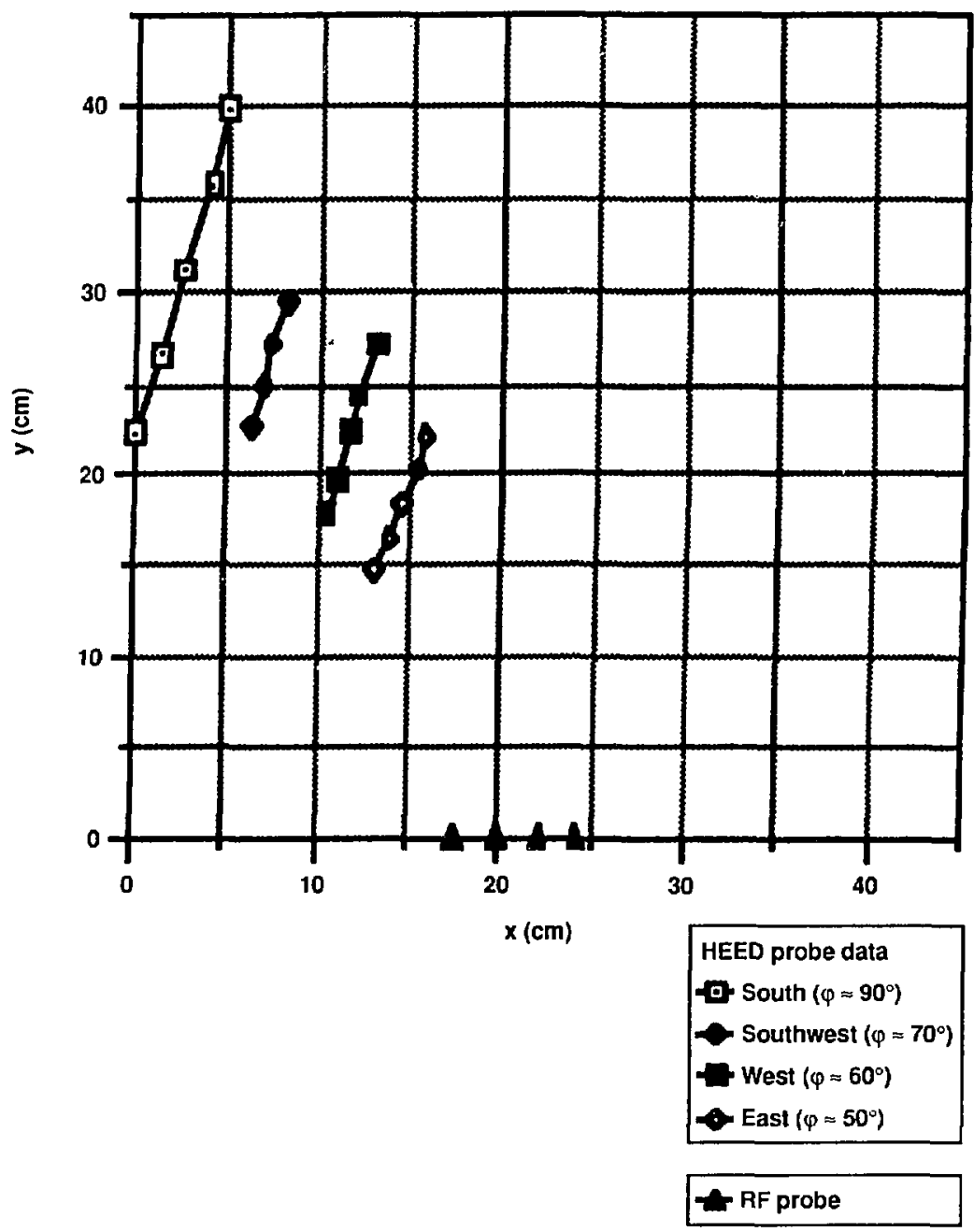

Figure 3.1. HEED probe limiter positions mapped to the West end-cell midplane. 
Both systems were operated for $55 \mathrm{~ms}$, but the $10-\mathrm{kG}$ power was modulated at 100 rate during this period to distinguish the effects of the two systems. Figure 3.2 shows typical incident power waveforms for the ECRH systems.

On 10/31/86, a radio-frequency $(R F)$ probe scan was pcrformed in both endcells. The RF probes of interest consist of multiple tungsten wire tips and are regarded here as plasma limiters. In the West, the probe inserts along the negative $\mathrm{x}$-axis $26 \mathrm{~cm}$ inboard from the Wesi end-cell magnetic midplane. TMX-U was operated under constant heating and fueling conditions similar to the $11 / 14 / 86$ run with three signinicant differences. First, the 5-kG ECRH was aimed at $\mathrm{z}=602$ $\mathrm{cm}$; second, the sloshing-ion neutral bearns were operated in both end-cells; third, a slightly different magnetic field case was used to accommodate the different ECRH aiming. Despite these differences, the hot-electron parameters measured during this operation are similar to those of the $11 / 14 / 86$ experiment; therefore, the results of two experiments have been combined to provide a complete map of the hot-electron halo in the West end-cell. Shot-to-shot reproducibility was not quite as good as during the 11/14/86 operation, so a subset of the data has been selected using the end-cell midplane line density as the criteria. Only shots with flat-topped line densities within $10 \%$ of $5.0 \times 10^{13} \mathrm{~cm}^{-2}$ are included in the subsequent analysis.

\subsubsection{Perpendicular Pressure Profile}

We examine the peak diamagnetism as a function of limiter probe position. Differentiation of the results with respect to cross-sectional area provide the total plasma pressure profile.

The diamagnetic loop signal suffers from noise problems introduced by ripple in the TMX-U confining magnetic field. Because of the design of the magnet regulation circuitry, the major ripple occurs at various frequencies near $120 \mathrm{~Hz}$. Beating between the similar frequencies produces low-frequency components in the loop voltage tlat, when integrated, cause a variable amount of baseline drift. This effect limits the accuracy of the diamagnetic loop system to $\pm 2 \times 10^{4} \mathrm{Acm}$.

The peak diamagnetism varied considerably during the limiter pi.. be scans. Diamagnetism versus HEED limiter radius is plotted in figures 3.3(a-d). For all 

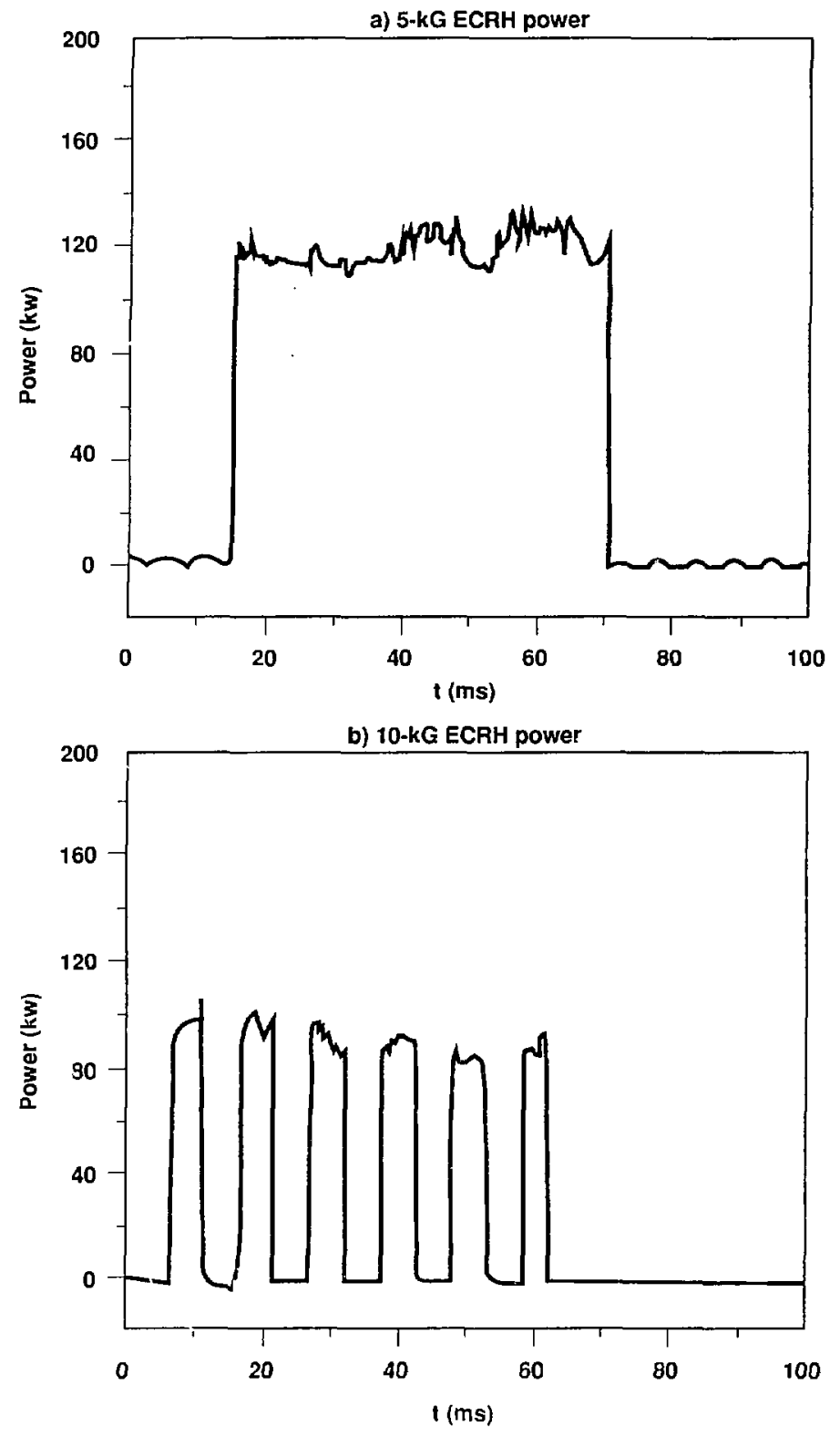

Figure 3.2. ECRH waveforms for the West end-cell systems on $11 / 14 / 86$; (a) 10-kG heater, (b) 5-kG heater. 
orientations, the data show a rapid drop to $40-50 \%$ of the peak value in the space of 2-8 cm. The shot-to-shot consistency of the diamagnetism is indicated in figure 3.4(a), which is a plot of the unperturbed (probe radius $\geq$ plasma boundary) peak diamagnetism versus shot number for the 11/14/86 experiment. The values are somewhat lower at the start of the first scan; thereafter they are quite constant. The initially low diamagnetism is caused by a fluctuation in the magnetic coil currents [see figure 3.4(b)]. The end-cell solenoid currents were reduced by $2 \%$ starting with shot 11 , but there was significant undershoot until shot 16 ; thereafter, the field :emains fairly constant.

The diamagnetism variation in the West end-cell measured during the 10/31/86 RF probe scan experiment is shown in figure 3.3(e). These data are consistent with the $11 / 14 / 86$ results. The peak unperturbed end-cell midplane diamagnetism was typically $3.6 \times 10^{5} \mathrm{Acm}$. Peak diamagnetism versus RF probe midplane radius is shown in figure $3.3(\mathrm{e})$. The decrease in diamagnetism is remarkably rapid, with a $40 \%$ reduction occurring within $2 \mathrm{~cm}$.

In the limiter probe scans, if the peak diamagnetism for each scan is $M_{\max }$, we can define a normalized diamagnetism by $\Delta M \equiv 1-\frac{M}{\boldsymbol{M}_{\max }}$. The normalized diamagnetism is interpolated using a straightforward cubic-spline technique ${ }^{17}$ to yield the locations of the $0 \%, 10 \%, 20 \%$, etc., scrape-off locations. We plot these locations in figure 3.5, along with ellipses fitted to each contour. The elliptical fit is suggested by the magnetic-curvature-drift surfaces, which are described in the section 3.2.2. The fit to the data is quite good, with the RMS error less than $1.5 \mathrm{~cm}$ in all cases. Note that the RF probe scan data of 10/31/86 have been combined with the 11/14/86 data. The two data sets, despite the different machine operating conditions, match very well. Altogether, the plasma boundary has an ellipticity of 1.5, while the inner contours are more circular.

Under the assumption of quadrupole symmetry, the diamagnetism contour data can be differentiated with respect to area to obtain an azimuthally averaged perpendicular pressure profile. Since the diamagnetism is

$$
M=\frac{1}{B_{0}} \int P_{\perp} d A
$$


(a) $\phi=90$ degrees

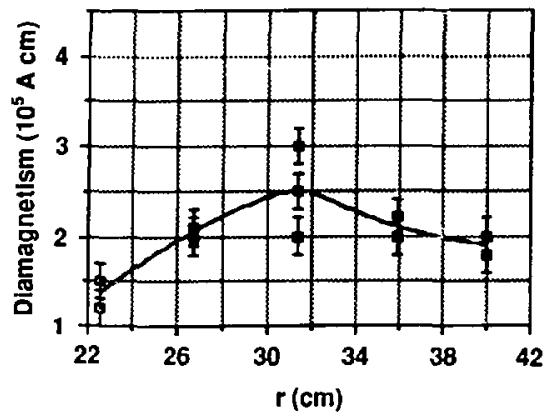

(c) $\phi=60$ degrees

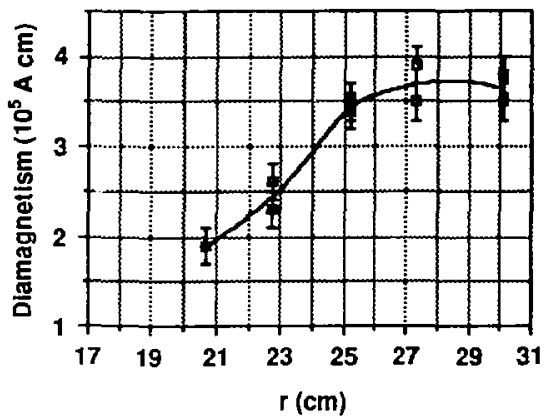

(b) $\phi=70$ degrees

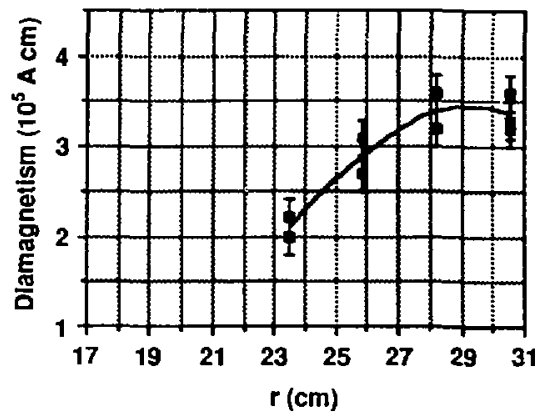

(d) $\phi=\mathbf{5 0}$ degrees

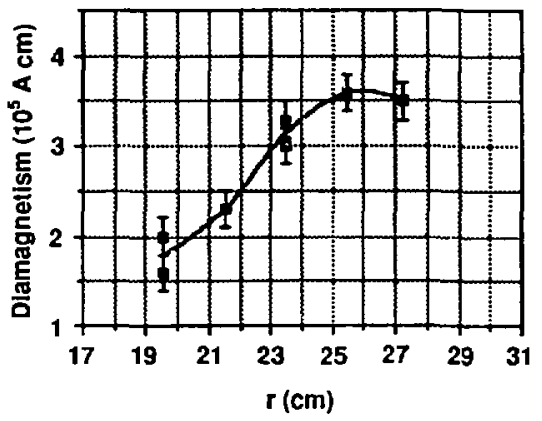

(e) $\phi=0$ degrees

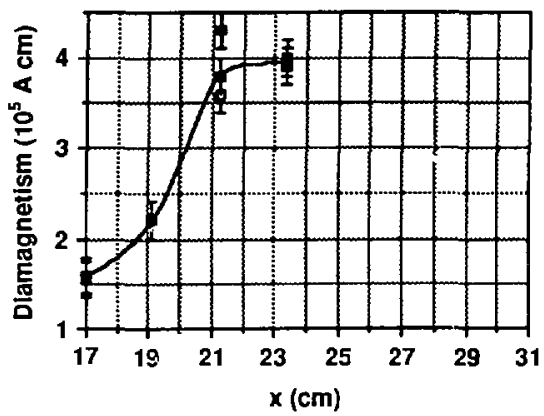

Figure 3.3. Peak diamagnet ism plotted for five limiter scans, phi values of (a) $90^{\circ}$, (b) $70^{\circ}$, (c) $60^{\circ}$, (d) $50^{\circ}$, and (e) $0^{\circ}$. 
a) Unperturbed dlamagnetism

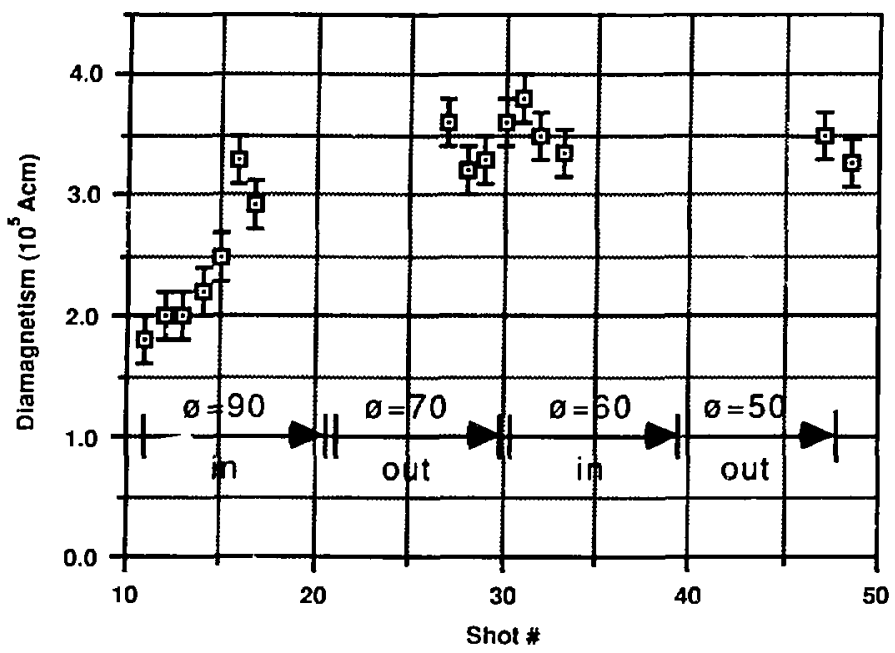

b) Midplane manetic fleld

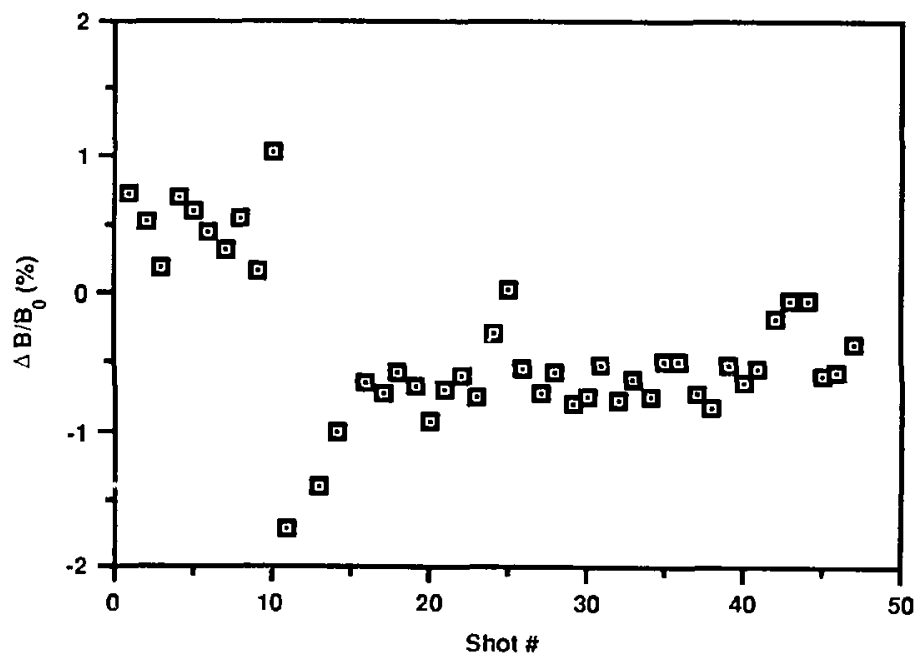

Figure 3.4. Limiter scan consistency: (a) unperturbed peak diamagnetism, (b) midplane magnet ic-field offset 


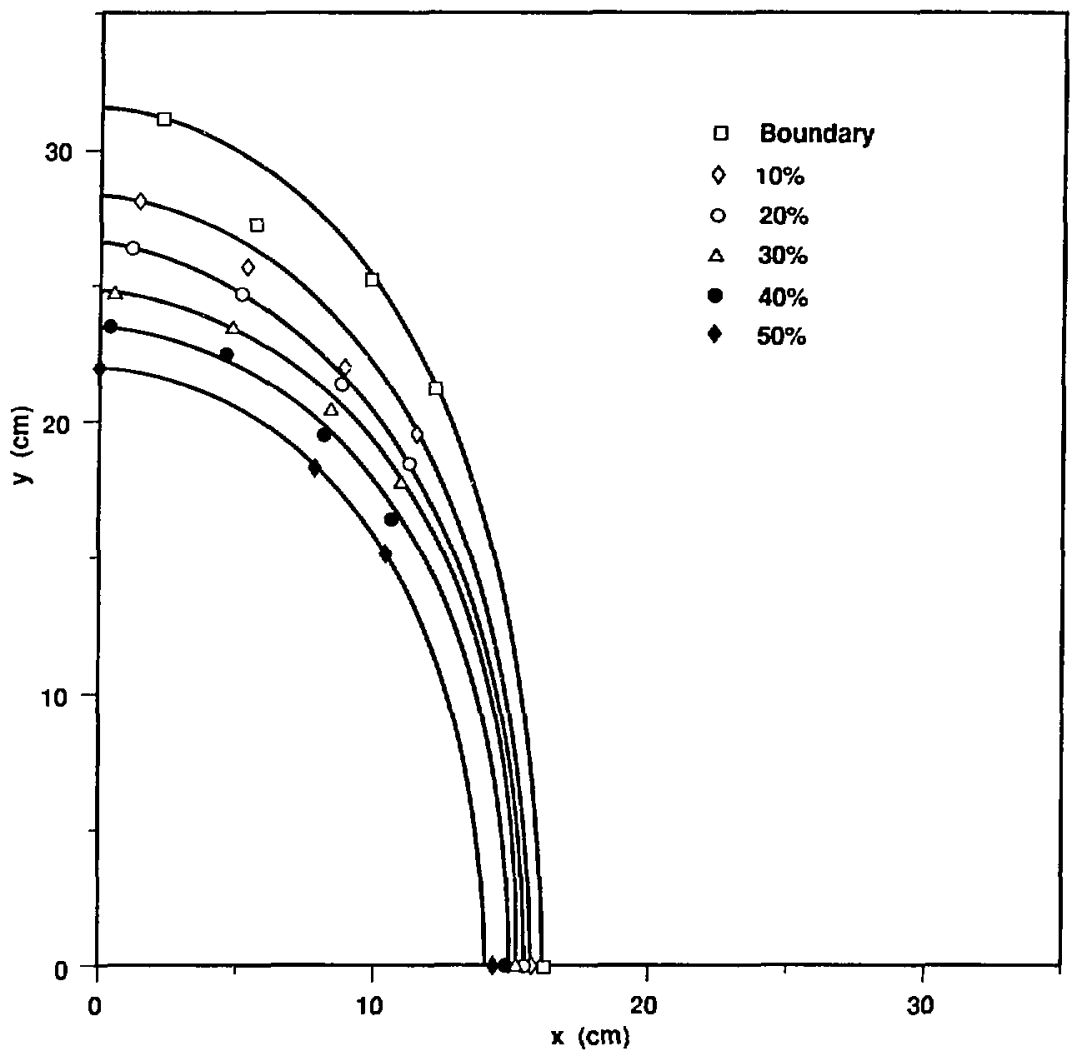

Figure 3.5. Contours of hot-electron energy-density. 


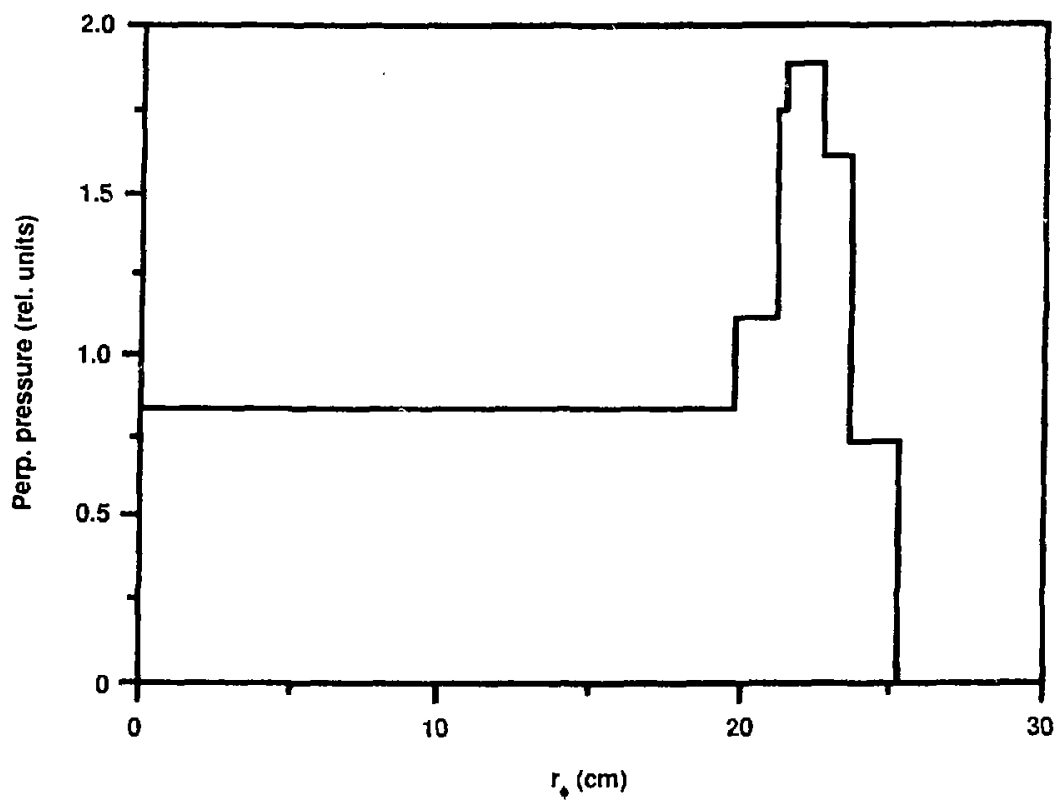

Figure 3.0. The azimuthally-averaged total perpendicularpressure profile in the West end-cell.

then the average pressure within the contour is

$$
<P_{\perp}>\equiv \frac{1}{\Delta A} \int P_{\perp} d A=\frac{B_{0} \Delta M}{\Delta A} .
$$

The values for $\Delta M$ and $\Delta A$ are obtained from figure 3.5 , where the difference between two contours is $\Delta M=10 \%$, and $\Delta A$ is the cross-sectional area between them. Since these results give the pressure averaged in azimuth, they should be plotted versus the average radius of the contour,

$$
r_{\phi}=\frac{1}{2 \pi} \int_{0}^{2 \pi} d \phi r(\phi) .
$$

The West end-cell total pressure profile, normalized to the average pressure, is plotted versus $r_{\phi}$ in figure 3.6(a). The profile is hollow, with the pressure in the plasma halo more than a factor of two larger than in the core region. 


\subsubsection{Comparison of the Magnetic-Curvature Drifts with the Pressure Profile}

Calculations of the magnetic-curvalure drift surfaces in TMX-U show a considerable deviation from the circular mod-B flux surfaces for a class of midplane pitch angles, as shown in figures $3.7(a-d)$. The calculation is carried out with the SPLINE guiding-center drift code, as described in appendix $A$. The pitch angle is defined by $\tan \theta=v_{\perp} / v_{\|}$. Deeply trapped particles with $\theta \geq 80^{\circ}$ have essentially circular drift orbits. However, as the particles bounce further from the midplane, they increasingly sample noncanceling geodesic curvature introduced by the transition coil set. As a result, their orbits become elliptical. This ellipticity reaches a maximum at about $65^{\circ}$, then decreases slowly with decreasing $\theta$. Note that in TMX-U, measurements of the axial variation of diamagnetism indicate a hot-electron length corresponding to a pitch angle of $65^{\circ}$. In the East end-cell, the drift surfaces are similar, simply rotaled $90^{\circ}$ in the $x-y$ plane.

For particles with midplane pitch angles less then $80^{\circ}$, the drift surfaces have an interesting structure. Near the magnetic axis, the orbits are fairly circular. With increasing radius, the trajectories become increasingly elliptical. Finally, at still larger radii, a separatrix is reached, beyond which the drift surfaces "open up," and no longer enclose the magnetic axis.

The scrape-off measurements show the spatial structure of the hot-electron halo in the West end-cell. The drifts of the energetic electrons within the halo are dominated by the magnetic curvature (see appendix A). Halo electrons are created by localized absorption of the 5-kG ECRH at the plasma boundary. Absorption is strong here because the diamagnetism of the plasma has shifted the second-harmonic resonance to this location. Thus, the elliptical plasma boundary corresponds to the outermost closed suriere of the $65^{\circ}$ pitch-angle drift surfaces, as shown in figure 3.8. Furthermore, the inner scrape-off contours are tightly spaced along the $\mathrm{x}$ axis due to the localized absorption of the ECRH. The spacing broadens towards the $y$ axis as electrons with various of pitch angles drift along different surfaces. The halo equilibrium is closely related to electron losses along the open drift surfaces, as discussed in section 4.4. The possibility of radial losses associated with open drift surfaces was first explored by Failor ${ }^{18}$. An analogous 


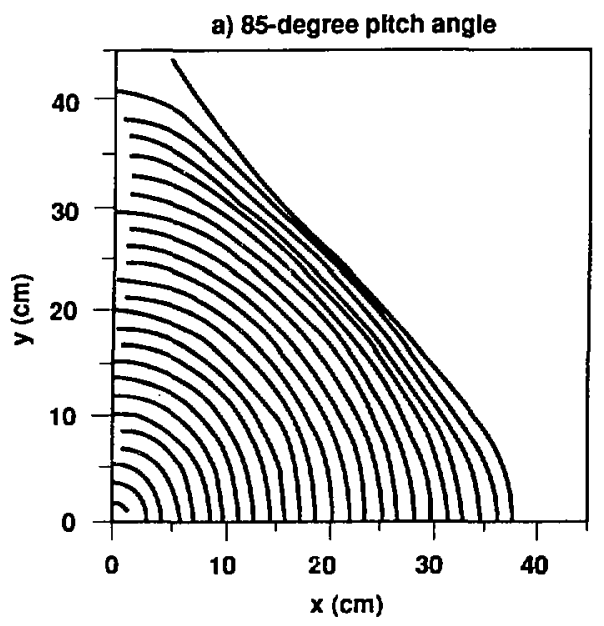

b) 75-degree pltch angle
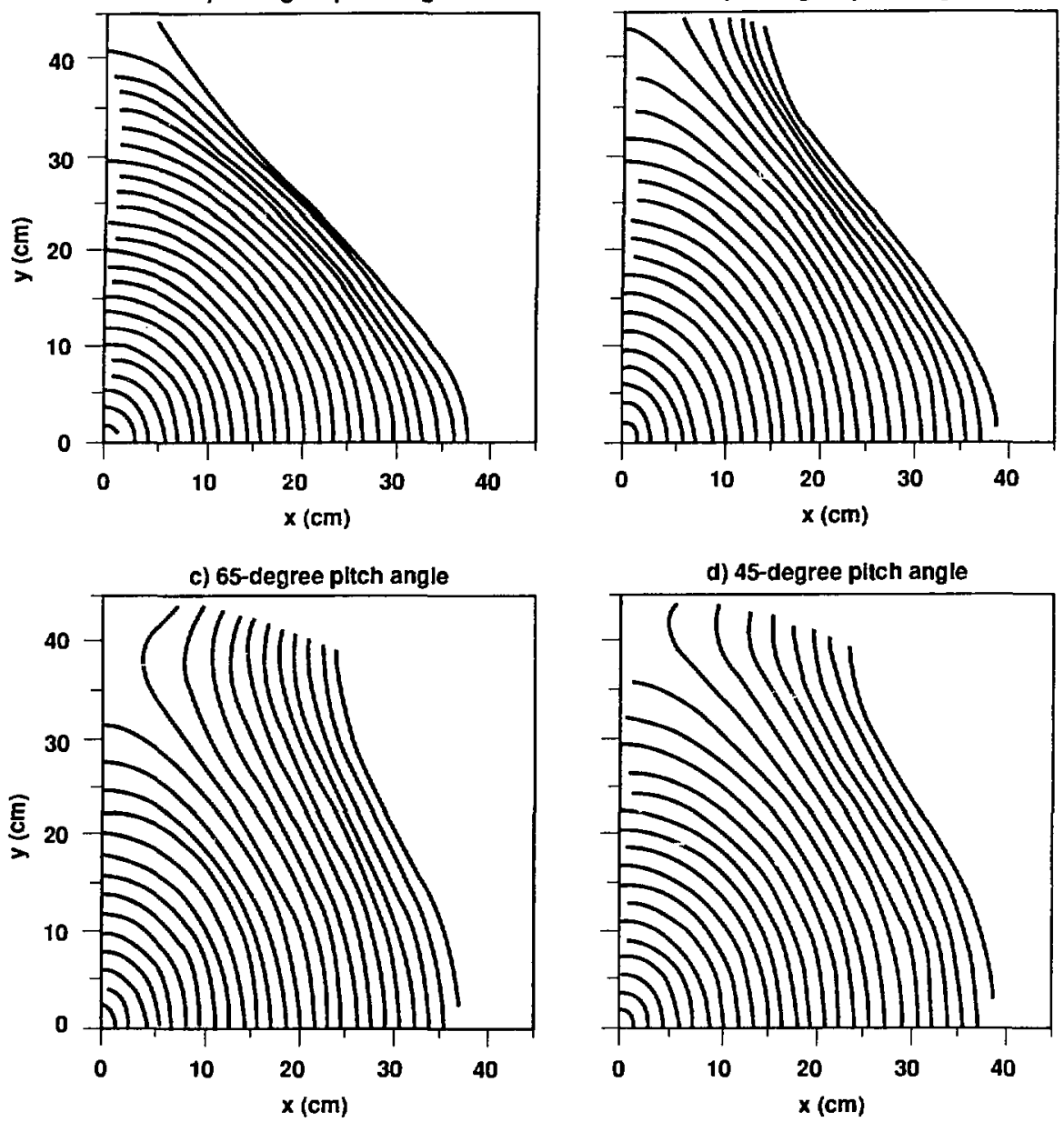

Figure 3.7. Curvature-Ibrift surfaces for various midplane pitch angles: (a) $85^{\circ}$, (b) $75^{\circ}$, (c) $65^{\circ}$, (d) $45^{\circ}$. 
drift-mapped annular plasnia structure has been observed in the Constance magnetic mirror ${ }^{19}$. In Constance, however, both the radial and axial magnetic wells are much narrower, and the mirror-trapped electrons trace out a "baseball seam"shaped structure as they drift. In TMX-U, even deeply trapped electrons have a significant bounce length, so the plasma structure is spread out axially.

In the East end-cell we expect the halo to have a different structure. Here, the 5-kG ECRH beam is again delivered along the $x$ axis, but the $c$ if i surfaces are rotated by $90^{\circ}$. Because the separatrix is now adjacent to the $y$ axis, the plasma can expand farther along the $x$-axis.

\subsubsection{Spatial Structure of the Super-Hct Component}

The PECE diagnostic provides information about the most energetic component of the electron distribution. Such emission is produced at microwave frequencies by the energetic plasma electrons as they gyrate in the confining magnetic field. We use the PECE data obtained during the limiter probe scans to study the spatial structure of the super-hot-component plasma.

On TMX-U, electron-cyclotron emission is measured at frequencies large compared to the local gyro-frequency. Specifically, PECE is measured at 98 and $60 \mathrm{GHz}$ in the West end-cell. For TMX-U plasma densities, this emission is optjcally thin and therefore is proportional to a line average of the product of density with a complex function of electron energy distribution ${ }^{25}$. The diagnostic makes use of a quasi-optical microwave system in the West end-cell with a well collimated and narrow receiving pattern. For simplicity, we consider the measurements to be simple line averages in subsequent analysis. The viewing angle js vertical at an axjal location $16 \mathrm{~cm}$ outboard from the end-cell midplane.

The PECE signals can be analyzed to obtain estimates of the super-hotcomponent electron temperature and line density. If we assume a constant radial temperature profile, the ratio of the optically thin emissions at the two different frequencies yields a time-resolved measure of the electron temperature. As the microwave transport system is not absolutely calibrated, determination of the PECE temperature requires normalization to the $\mathrm{x}$-ray spectrometer measurement of the super-hot-component temperature in the afterglow plasma that typically persists 

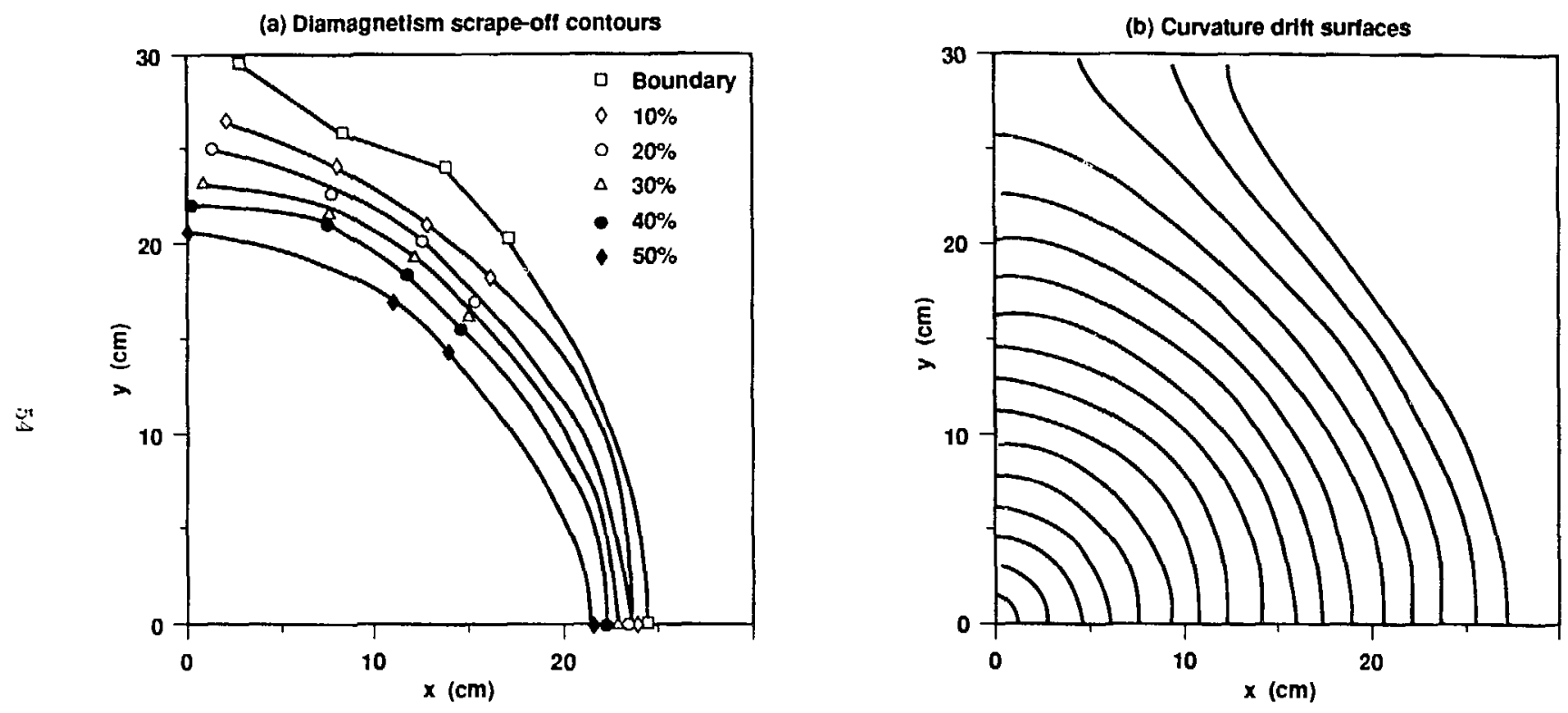

Figure 3.8. Comparison of the diamagnetism scrape-off contours with the $65^{\circ}$ pitch-angle drift orbits. 
long after all power systems have been turned off ${ }^{20}$. Since the emissions are optically thin, they are also proportional to the line-integrated dersity of the hottest electron component. This line density is obtained from the 98-GHz PECE signal by correcting for the temperature:

$$
<n_{l, h o t}>\equiv \frac{\int n_{h o t} F\left(T_{h o t}\right) d l}{F\left(T_{h o t}\right)},
$$

where the numerator is proportional to the raw PECE signal, and the temperature is obtained from the ratio of the $98-\mathrm{GHz}$ signal to the $60 \mathrm{GHz}$ signal as ciescribed. This result becomes the exact hot-component line density when the temperature profile is constant. Again, the PECE system is not absolutely calibrated, and this measurement of the line density is only relative.

Figures 3.9(a-e) show the PECE temperature at ECRH turn-off as a function of limiter radius for the five scans. There is only a very small variation with probe position. The temperature is less than $10 \%$ lower when the probe is fully inserted; thus, the assumption of a uniform temperature profile is justified, at least in the halo region. The average temperature is $270 \mathrm{keV}$.

In figures 3.10(a-e), we plot the values of the PECE $98-\mathrm{GHz}$ and $60 \mathrm{GHz}$ signals at the end of the 5-kG ECRH pulse versus limiter radius for the five probe scans in the West end-cell. All of the scans show a drop of $40-50 \%$ as the limiter is inserted to its maximum extent. The unperturbed values are plotted versus shot number for the $11 / 14 / 86$ data in figure 3.11 . The peak value rises somewhat during the first few shots of the run, which is consistent with the diamagnetism data (see figure 3.4).

If the super-hot component temperature is uniform, the PECE signals provide a relative measure of the super-hot component line density. For the probe scan along the $y$ axis, we differentiate the normalized PECE 98-GHz signal to obtain the density profile at the $90^{\circ}$ azimuth. The profile, shown in figure 3.12(a) shows a slight hollowness of about $30 \%$.

To continue the analysis, we need a model for the hot-electron plasma azimuthal variation. The close agreement between the curvature drift orbits and the observed perpendicular pressure profile suggests a drift mapping. Also, if the hot-component temperature is constant, the density and pressure have the same structure. Under the drift-mapping assumption, flux is conserved along drift or- 
(a) $\phi=90$ degrees

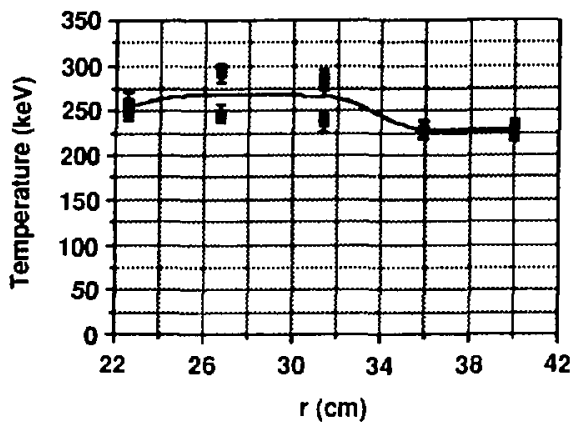

(c) $\phi=\mathbf{6 0}$ degrees

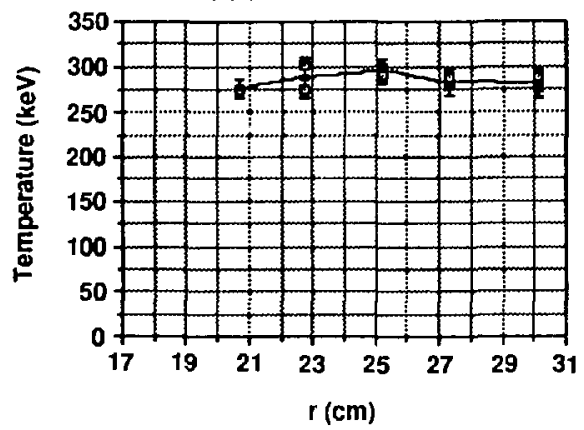

(b) $\phi=70$ degrees

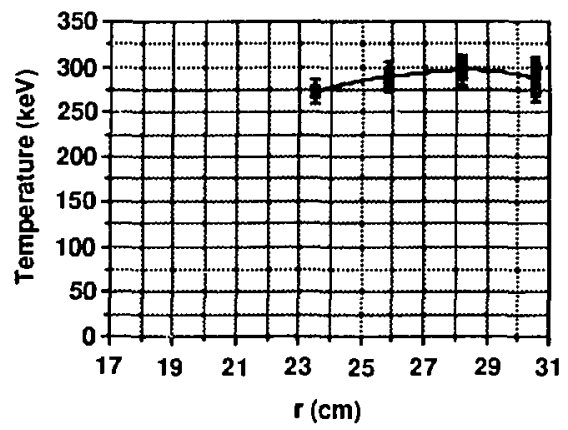

(d) $\phi=\mathbf{5 0}$ degrees

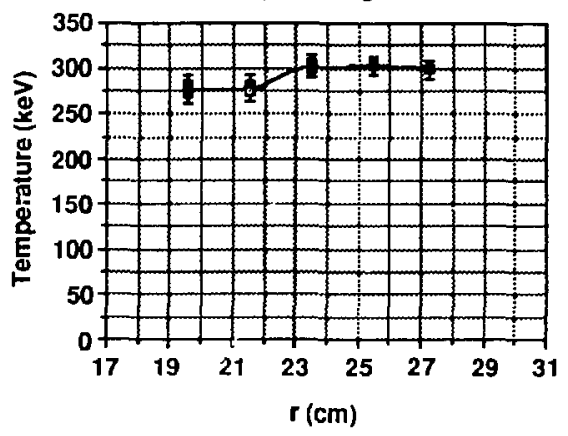

(e) $\phi=0$ degrees

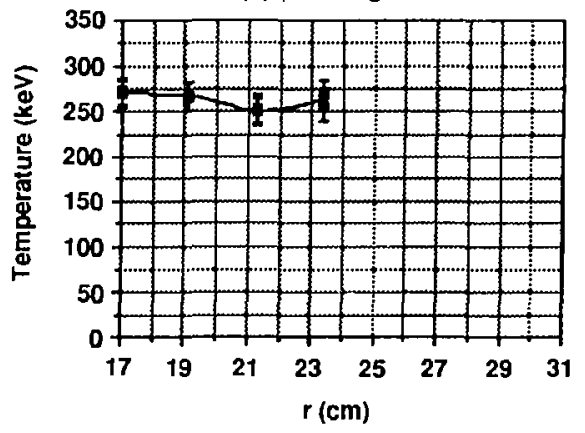

Figure 3.9. Spatial variation of the PEC.F temperature; (a d) HELI) probe scans, and (e) $\mathrm{KF}^{\mathrm{r}}$ probe scan. 
(a) $0=90$ degrees

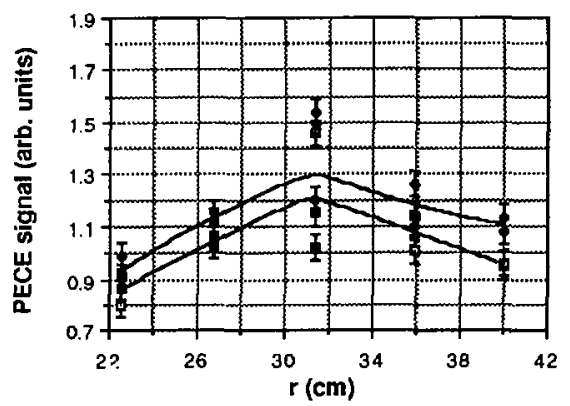

(c) $\sigma=60$ degrees

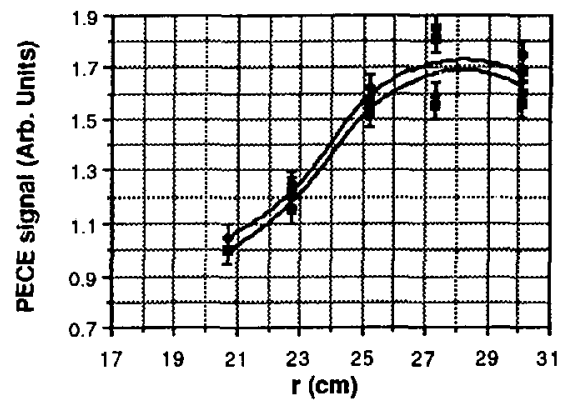

(b) $6=70$ clegrees

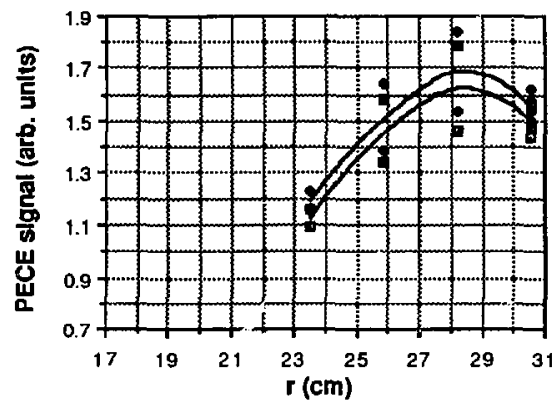

(d) $\sigma=50$ degrees

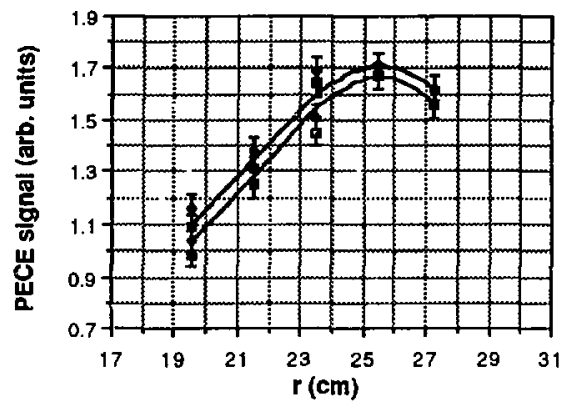

e) $\phi=0$ degrees

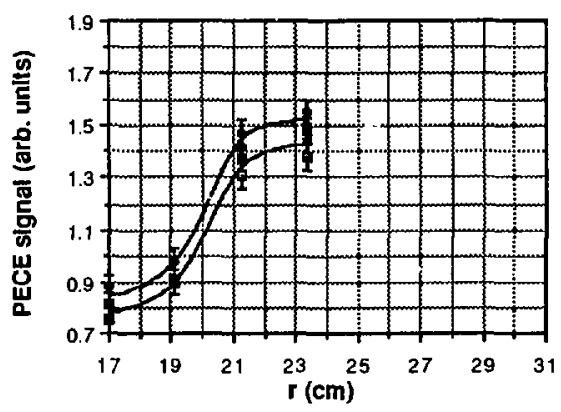

- $98 \mathrm{GHz}$

- $60 \mathrm{GHz}$

Figure 3.10. Spatial variation of the 98 and $60 \mathrm{GHz}$ PECE signals; (a-d) HEED probe scans, and (e) RF probe scan. 


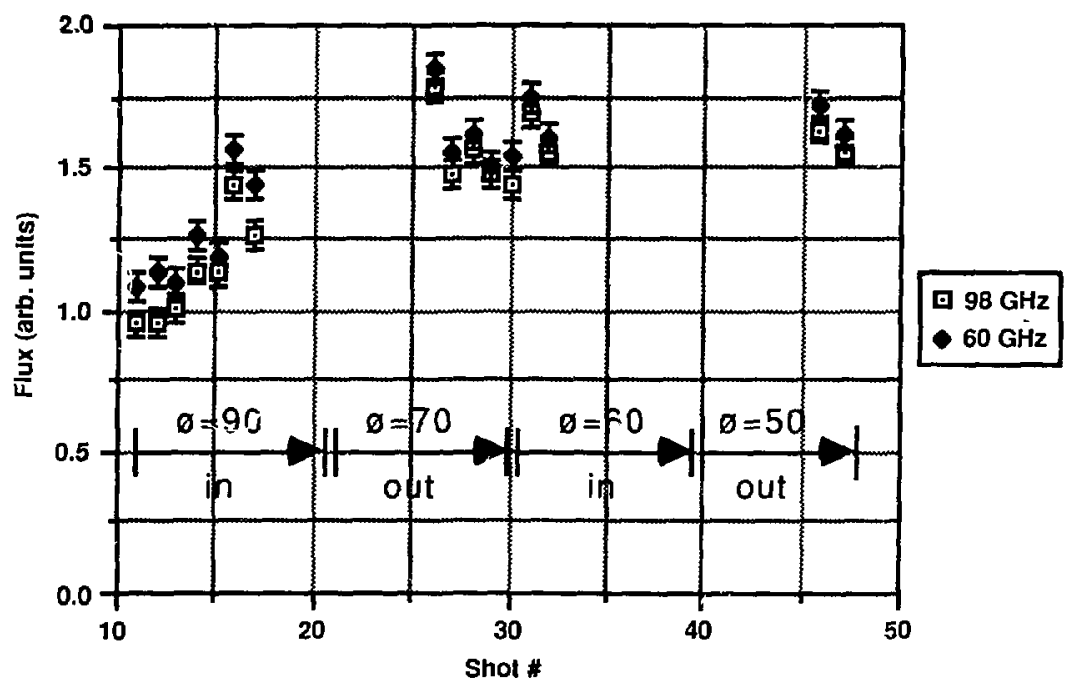

Figure 3.11. Thie unperturbed 98 and $60 \mathrm{GHz}$ PECE signals as a function of shot-number for the $11 / 14 / 86$ experiment. 


\section{(a) $\phi=90$ degrees}

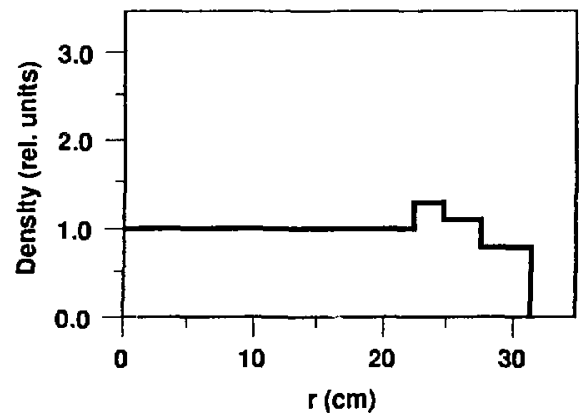

(b) $\phi=50$ degrees

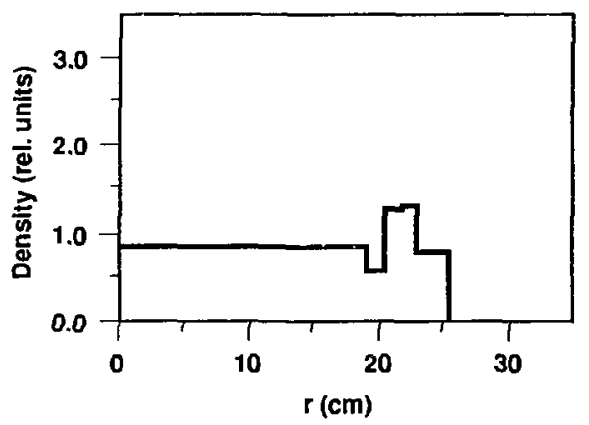

(c) $\phi=0$ degrees

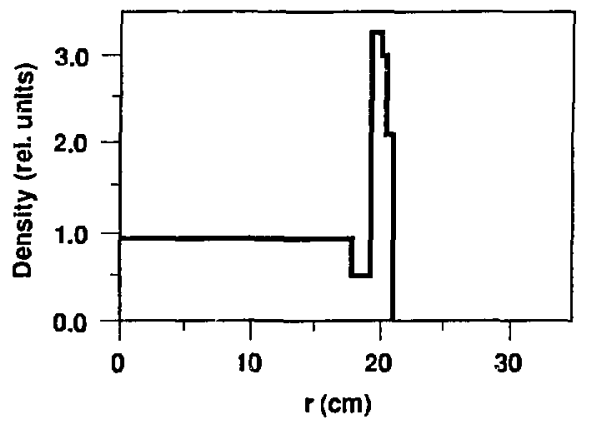

Figure 3.12. The super-hot component density profile for various azimuths; (a) $90^{\circ}$, (b) $50^{\circ}$, and (c) $0^{\circ}$. 
bits:

$$
n_{e}\left(\phi_{1}\right) v_{d r i f t}\left(\phi_{1}\right) \Delta A_{d r i f t}\left(\phi_{1}\right)=n_{e}\left(\phi_{0}\right) v_{d r i f t}\left(\phi_{0}\right) \Delta A_{d r i f t}\left(\phi_{0}\right),
$$

where $\Delta A_{d r i f t}$ is the cross-sectional area of a "drift tube." Assume further that the drifts are caused entirely by vacuum magnetic-field curvature; the drift velocities can then be calculated with the SPLINE code.

The drift-mapping model also allows us to determine a hot-electron pitch-angle distribution. The contours of PECE scrape-off, fitted to ellipses as with diamagnetism data and shown in figure 3.13, again indicate the presence of the elliptical and annular hot-electron halo. Since the halo emanates from a narrow region on the $\mathrm{x}$ axis (due to the beta-depressed second-harmonic resonance), we can obtain the pitch-angle distribution of the halo plasma froms the $y$-axis hot-electron density profile in the following manner. Each point in the profile corresponds to a specific eccentricity referred to the plasma boundary on the $x$ axis. Knowledge of the curvature drift orbits allows us to associate the eccentricity with a pitch angle, which completes the mapping. The relationship between eccentricity and pitch angle is shown in figure 3.14. Note that we must assume the distribution does not extend beyond $55^{\circ}$ to allow a unique unfold; this assumption is reasonable in view of the measured hot-electron length. Applying this procedure yields the pitch-angle distribution shown in figure 3.15. The distribution is peaked at about $75^{\circ}$, and is cut off at $55^{\circ}$ by assumption. Although very rough, this result is consistent with the Fokker-Planck calculations summarized in section 4.4.4.

With the drift-mapping assumption, the PECE limiter scan data for the various orientations give $A_{\text {drift }}$ under the assumption of a constant hot-electron length, and we can now generate profiles of the super-hot component density corresponding to various azimuths. The results are thown in figures $3.12(b-c)$. Note that since the temperature is uniform, these are also plots of the relative super-hot-component pressure. The profiles all show some degree of hollowness.

To allow comparison of the super-hot-component and total pressure profiles, we mus' integrate the PECE pressure profile in azimuth using the elliptical fits to produce a continuous function for $A_{d r i f t}(\phi)$. The result, shown in figure 3.16 , is similar to the measured total pressure profile previously shown in figure 3.6 . In section 3.2.4 the two profiles are compared quantitatively. 


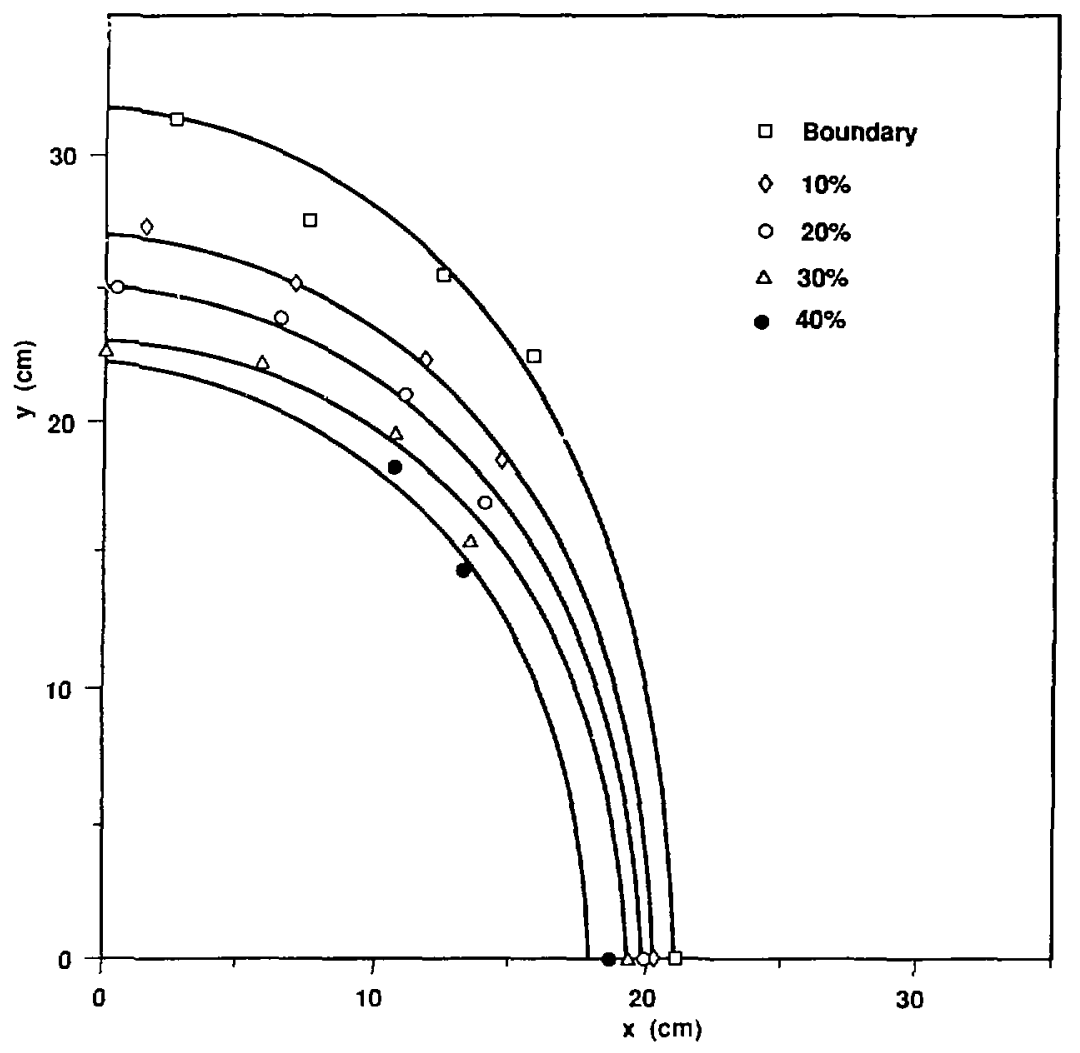

Figure 3.13. $98 \mathrm{GHz}$ PEC.F, scrape-off contours. 


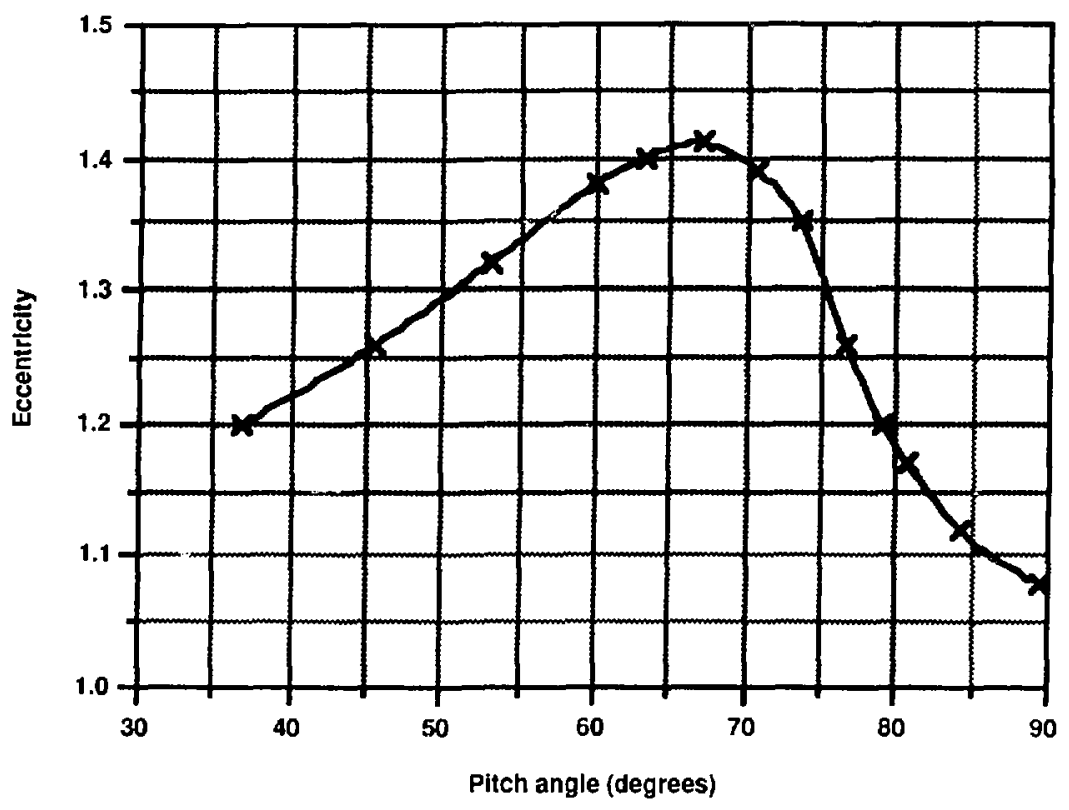

Figure 3.14. Drift orbit eccentricity versus midplane pitch-angle. 


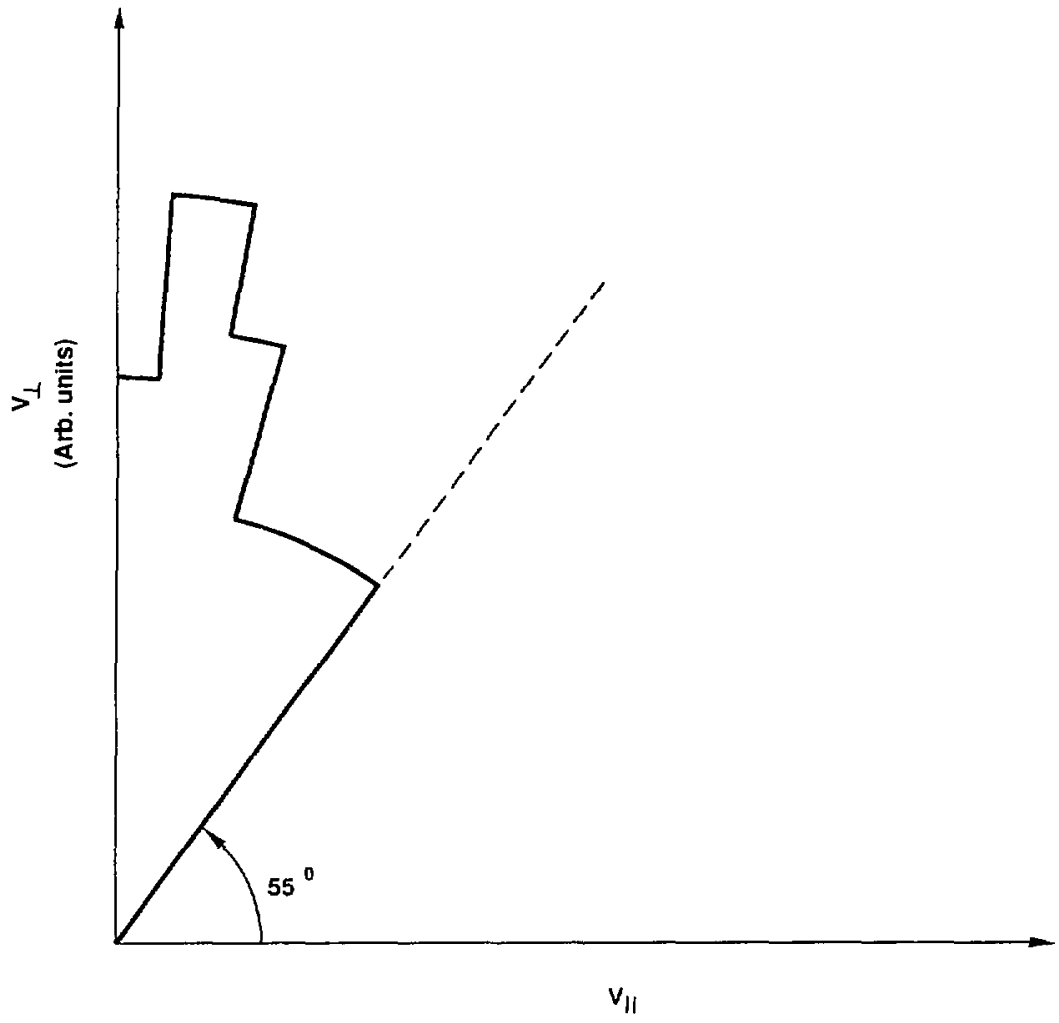

(Arb. units)

Figure 3.15. Hot-electron pitch angle distribution infersed from the PECE, scrape-off contours. 


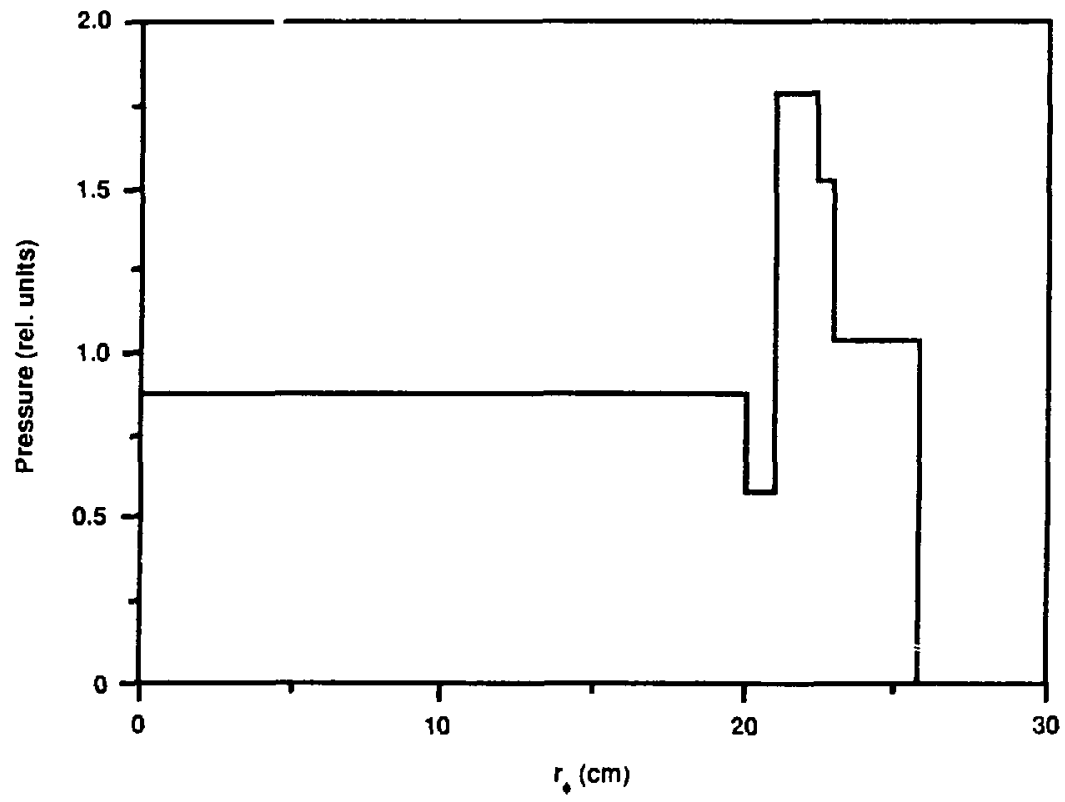

Figure 3.16. The azimivthally averaged super-hot component pressure profile in the West end-cell. 


\subsubsection{Spatial Structure of the Hot Component}

Comparison of the total- and super-hot-component pressure profiles provides information about the spatial structure of the hot-component plasma. Even allowing significant errors in these measurements, it is apparent that the hot component must be present largely in the outer regions of the plasma. To quantify the magnitude of the hot component, absolute numbers must first be assigned to the total and super-hot pressure profiles for some particular shot; their difference yields the average hot-component pressure. The temperature measured by the HEED probe or low-energy $x$-ray spectrometer, along with an estimate of the anisotropy, will then permit an estimate of the hot density.

We can assign absolute magnitudes to the total- and super-hot-component pressure profiles by examining the late-time behavior of the diamagnetic loop. As noted in section 1.2, the diamagnetism falls by about $10-20 \%$ in the first $10 \mathrm{~ms}$ after the ECRH is turned off. The residual diamagnetism that persists is the long-lived super-hot component, and the peak diamagnetism corresponds to the total pressure. To take a specific example, we examine shot 32 of 11/14/86, where the limiter probe was fully retracted. Here, the peak diamagnetism was $4.2 \times 10^{5} \mathrm{Acm}$, or $21 \mathrm{~J} / \mathrm{cm}$. The average total perpendicular pressure is then

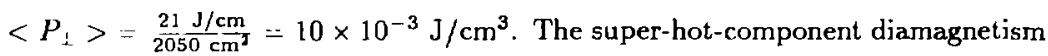
is $3.7 \times 10^{5} \mathrm{Acm}$, so the average super-hot-component pressure is $9 \times 10^{-3} \mathrm{~J} / \mathrm{cm}^{3}$. Taking the difference between the two profiles gives the hot-component profile. The total-, super-hot-, and hot-component pressure profiles are plotted together for comparison in figure 3.17. The majority (about 70\%) of the hot-component stored energy is within the outer elliptical annulus of the plasma. The peak magnitude of the hot-component pressure is about $4,10^{-3} \mathrm{~J} / \mathrm{cm}^{3}$. For this discharge, both the HEED probe and the $x$-ray spectrometer give a temperature of $25 \mathrm{keV}$ for the hot component. The peak hot-component density can be calculated from

$$
n_{\text {hot }}-\frac{P_{\perp}}{<\sin \theta>T_{h a t}} .
$$

The term $\langle\sin \theta>$ is obtained by averaging over the pitch-angle distribution. Using the experimentally measured distribution already presented, we get $\langle\sin \theta\rangle$ $=0.77$. Thus, $n_{\text {hot }} \approx 1.3 \times 10^{12} \mathrm{~cm}^{3}$. 'The azimuthally averaged hot density is a 


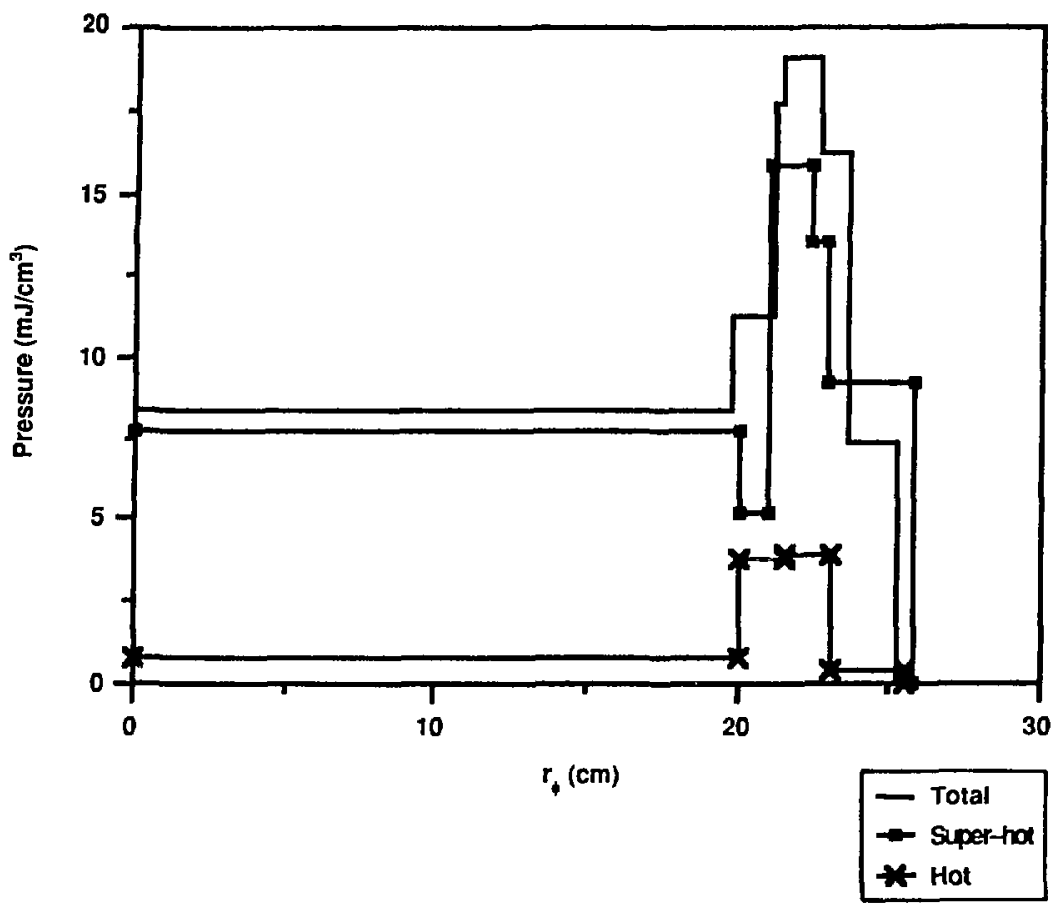

Figure 3.17. Components of the perpendicular pressure for $11 / 14 / 86$ shot 32 .

factor of four larger than the density of the super-hot component. However, like the super-hot component, the hot-component electrons should be dominated by curvature drift, and their density will change significantly with azimuth, becoming less dense along the $y$ axis. Ignoring the small hot-component density in the core region, the average line density of the hot component is $5 \times 10^{12} \mathrm{~cm}^{-2}$.

\subsubsection{The Cold and Warm Plasma Components}

Of the various limiter scan experiments, only the data of $11 / 14 / 86$ are examined; uncontrolled shot-to-shot variations obscure the results in the 10/31/86 scan. Typical line density time histories are shown in figure 3.18 for two shots. Figure 3.18(a) is shot 47 with the probe fully retracted, while $3.18(\mathrm{~b})$ is shot 42 with the probe fully inserted. In the unperturbed case, the line derisity increases $2025 \%$ when

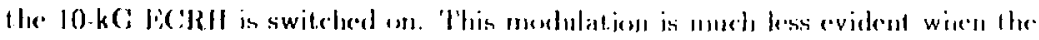


probe is fully inserted. In subsequent analysis, the values of the line density given are a temporal average from 30 to $60 \mathrm{~ms}$. As a measure of the shot-to-shot consistency of the scan, the unperturbed (limiter at maximum radius) line density is plotted as a function of shot number, as shown in figure 3.18(c). The unperturbed line density remains fairly constant throughout the experiment.

The total line density varied only slightly as a function of probe radius. Three of the four scans indicate a small reduction as the probe was moved inward. Figures $3.19(\mathrm{a}-\mathrm{d})$ shows the midplane line density as a function of the limiter midplane radius for the four limiter orientations. The data for the South orientation is noisy, which could be attributed to machine "clean-up" as this scan was taken first, early in the sun. The: average drop in line density is about $6 \times 10^{12} \mathrm{~cm}^{-2}$, or $12 \%$ of the peak value.

'The total line density measured at the end-cell midplane can be separated into two components. To proceed, we assume that the inboard interferometer measures only the coldest component of the plasma. The inboard interferometer is sufficiently far from the midplane (see section 1.3.1) to be largely unaffected by the more energetic, mirror-confined plasma. The cold-component line density thus measured can be flux-rnapped to the midplane and subtracted from the total line density measured by the midplane interferometer; the residue is an independent measurement of the mirror-trapped plasma line density.

The results of this separation are also shown in figure 3.19. The coldcomponent line density (labeled "streaming" in the figure) remains nearly constant as the limiter position is varied. Thus, the drop in the total line density is revealed as scrape-off of mirror-confined electrons. This drop is in good agreement with the measurement of the hot-component line density in section 3.1.5. Thus, the halg plasma appears to consist largely of energetic electrons, while the cold component is principally within the core region. 'This observation is consistent with previous measurements of cold plasma profile within the center cell ${ }^{21}$. The core region electrons are less energetic because they are strongly fueled from the center-cell gas box while poorly heated because the magnetic field depression produced by the super-hot electrons has forced the second-harnonic: resonance into the halo region. Conditions are reversed in the halo region, where fueling is due only to wall reflux, but strong heating is available berause the second-harmonic 
a) Time hlstory-probe retracted

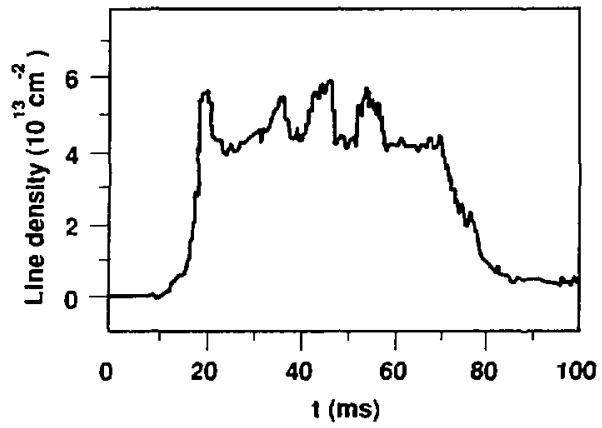

b) Time history-Probe inserted

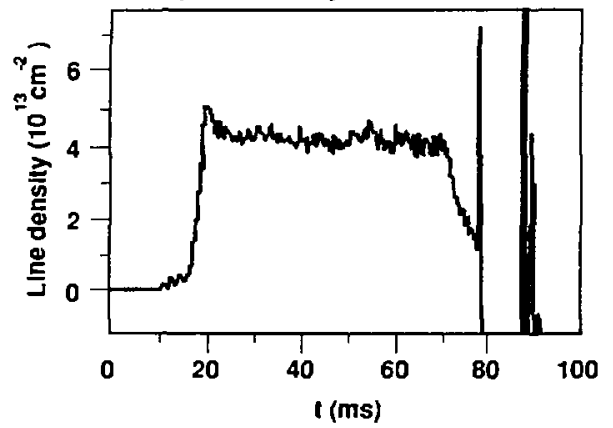

c) Unperturbed-line density

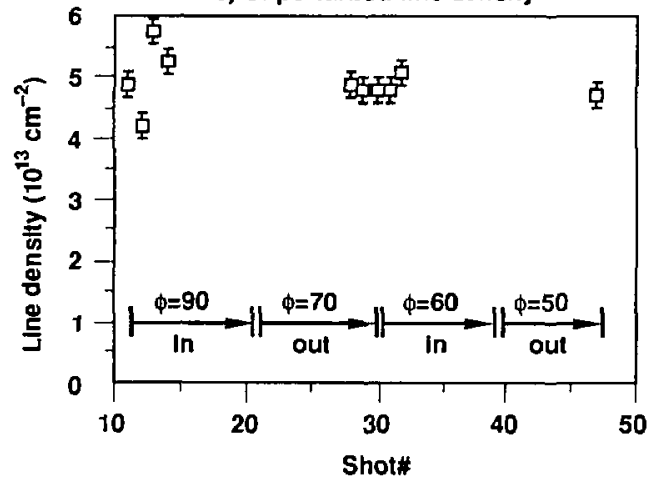

Figure 3.18. Iine-density data from the limiter-scan experiment: (a) time history -probe retracted; (b) time history probe fully inscrted; (c) linperturbed average line density versus shot mumber. 
a) $\phi=\$ j$ degrees

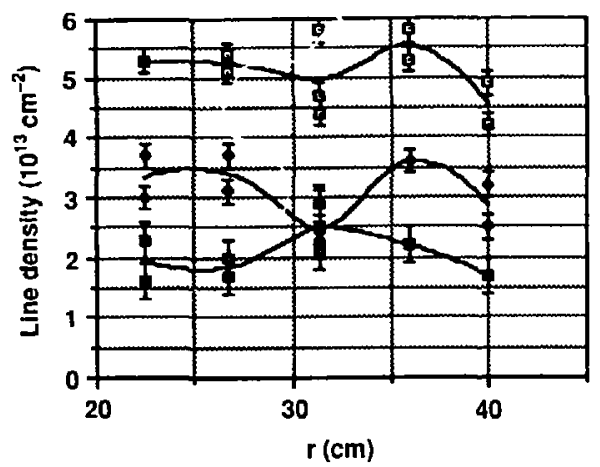

c) $\phi=60$ degrees

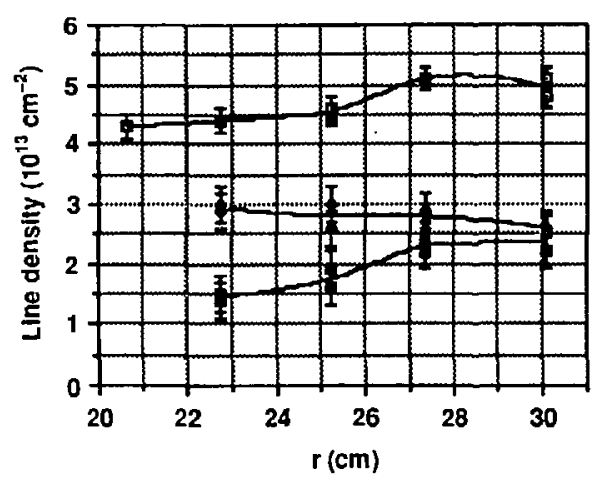

b) $\phi=70$ degrees

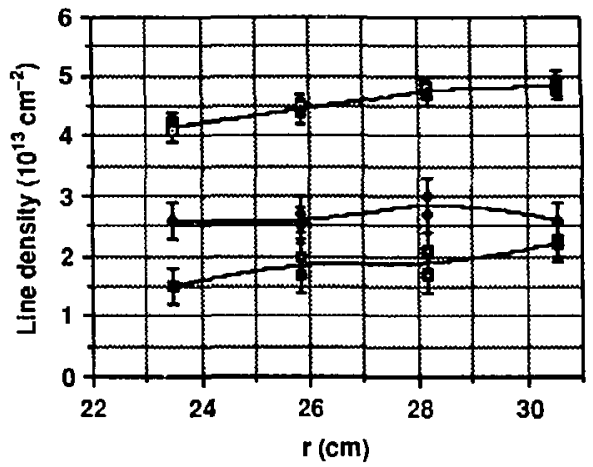

d) $\phi=\mathbf{5 0}$ degrees

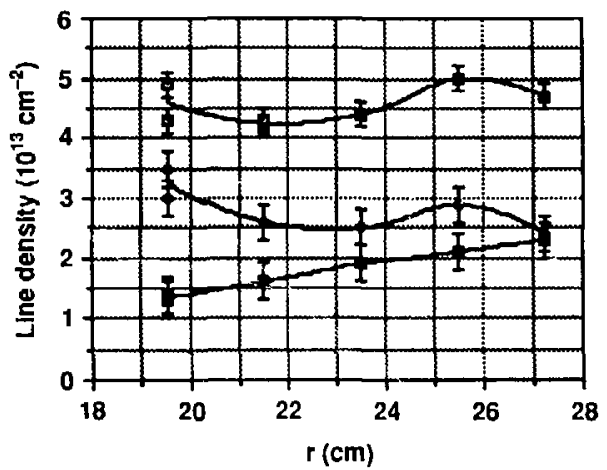

- Total

- Streaming

- Trapped

Figure 3.19. Plasma line-density data for the four HEED probe scans, $\phi$ values of: (a) $90^{\circ}$, (b) $70^{\circ}$, (c) $60^{\circ}$ and (d) $50^{\circ}$. 
resonance is present.

When the limiters are inserted to their minimum radius, about $60-70 \%$ of the mirror-trapped line density is not scraped off. The super-hot-component line density in the core region is very small, and the hot component is apparently present only in the edge region. Therefore, this additional mirror-trapped plasma must be in an intermediate energy range: sufficiently energetic to be effectively mirror-trapped, but cool enough to go undetected by both diamagnetisra and $\mathrm{x}$-ray spectrometer measurements. This plasma is the warm component. Its presence in the core region is shown plainly in spectra obtained by both of the end-loss spectrometers on the East fan, samples of which are shown in figures 3.20 (a) and (b). These spectra show somewhat cooler temperatures than typically observed. However, they are useful because they provide the only simultaneous measurements of the warm component by both the gridded electrostatic end-loss analyzer (ELA) and the solid-state electron end-loss spectrometer (EELS), both of which are described in section 4.3. The ELA spectrum shows the dominant end-loss current to be the cold-electron component, which typically has a temperature in the range of $50-200 \mathrm{eV}$, but it also clearly shows the more energetic warm-electron component with a temperature of about $1.8 \mathrm{keV}$. The EELS spectrum also shows the warm-electron component at about the same temperature. The simultaneous measurements shown in figures $3.20(a)$ and (b) were obtained during operation where $T_{e}$ was lower than normal due to poor vacuum conditions. More typically, the EELS measures a warm component temperature of approximately $3 \mathrm{keV}$, as shown in figure $3.20(\mathrm{c})$.

\subsubsection{Summary of the Plasma Structure}

The previous analysis shows a plasma with two distinct spatial regions: a halo and a core. The characteristics of the two regions as measured in the West end-cell are summarized in tables 3.1 and 3.2 .

The West end-cell halo is an elliptical annulus bounded between ellipses, 31 by $22 \mathrm{~cm}$ and 21 by $19 \mathrm{~cm}$. The halo csnsists entirely of energetic electrons, the hot and super-hot plasma components, with typical temperatures of 30 and $270 \mathrm{keV}$, respectively. The super-hot component dorninates the plassna pressure within this 


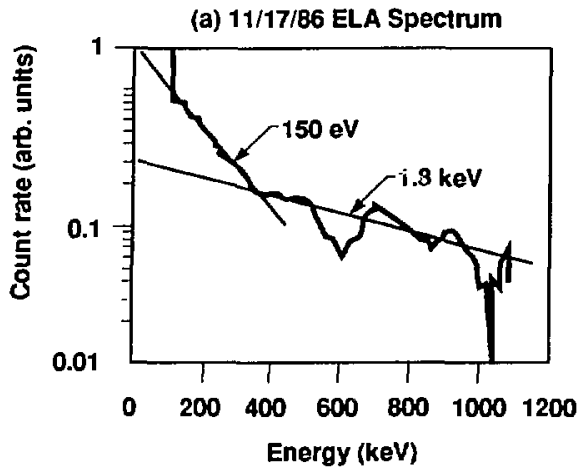

(b) 11/17/86 EELS Spectrum

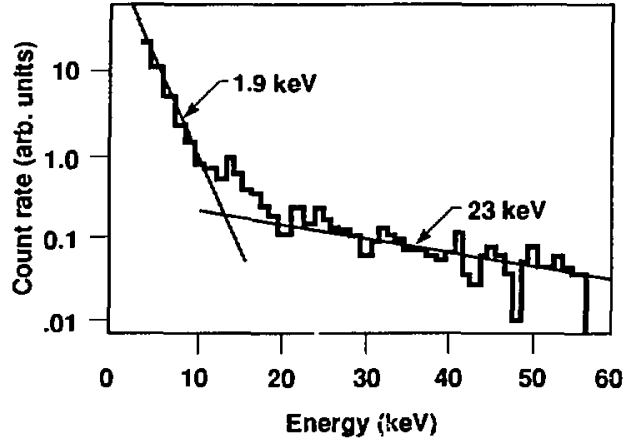

(c) 10/30/86 EELS Spectrum

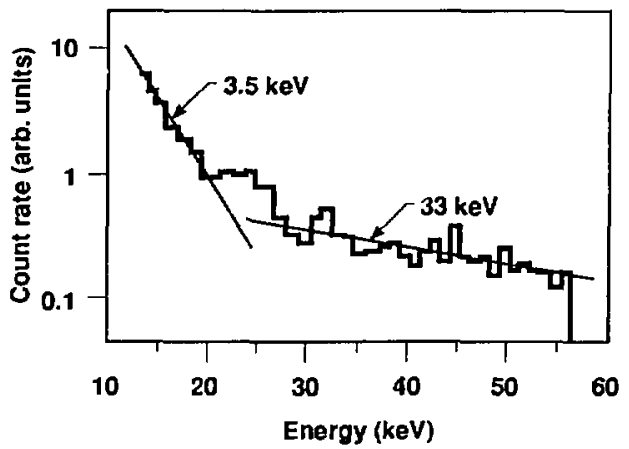

Figure 3.20. Electron end-loss spectra measured on the East fan by the (a) ELA, (b-c) EELS. 


\begin{tabular}{|c||c|c|}
\hline Component & Temperature (keV) & Density $\left(10^{11} \mathrm{~cm}^{-3}\right)$ \\
\hline Super-Hot & 270 & 4.0 \\
\hline Hot. & $20-50$ & 13 \\
\hline Warm & $2-5$ & small \\
\hline Cold & 0.1 & small \\
\hline
\end{tabular}

Table 3.1. Halo region plasma parameters in the West end-cell.

\begin{tabular}{|c||c|c|}
\hline Component & Temperature $(\mathrm{keV})$ & Density $\left(10^{11} \mathrm{~cm}^{-3}\right)$ \\
\hline Super-Hot & 270 & 0.8 \\
\hline Hot & $20-50$ & $<1$ \\
\hline Warm & $2-5$ & 3.5 \\
\hline Cold & 0.1 & 6.0 \\
\hline
\end{tabular}

Table 3.2. Core region plasma parameters in the West enci-cell.

region but at a lower density $\left(3 \times 10^{11} \mathrm{~cm}^{-3}\right)$, while the hot component is much more dense $\left(1.3 \times 10^{12} \mathrm{~cm}^{-3}\right)$. Magnetic-curvature drifts dominate the dynamics of these essentially collisionless electrons, and a drift-mapping model indicates that densities are a factor of two to three higher at the $\mathrm{x}$ axis than at the $\mathrm{y}$ axis.

The plasma spatial structure in the two end-cells can be explained by the interaction of localized ECRH at the beta-shifted second-harmonic resonance with the magnetic-curvature drift orbits. Due to the strong plasma beta, ECRF heating is localized near the plasma boundary along the $\mathrm{x}$ axis In the West end-cell, when $|x|<21 \mathrm{~cm}$, eiectrons of all pitch angles drift on closed surfaces (see figure 3.21 ). Beyond $21 \mathrm{~cm}$, electrons with a class of pitch angles near $65^{\circ}$ are lost on open cirift surfaces. Radial transport drives electrons onto the open surfaces, resulting in poor confinement and limiting the spatial extent of the plasma. When the ECRF power deposition is strongly localized, the halo is essentially the region between the plasma boundary and the inscribed circle.

Within the inner boundary of the halo region is the plasma core. Here again, the pressure is dominated by a small density of super-hot electrons. However, most of the core electrons fall into two categories: the cold-component plasma, with a temperature of about $100 \mathrm{eV}$, and the more energetic mirror-trapped warm- 


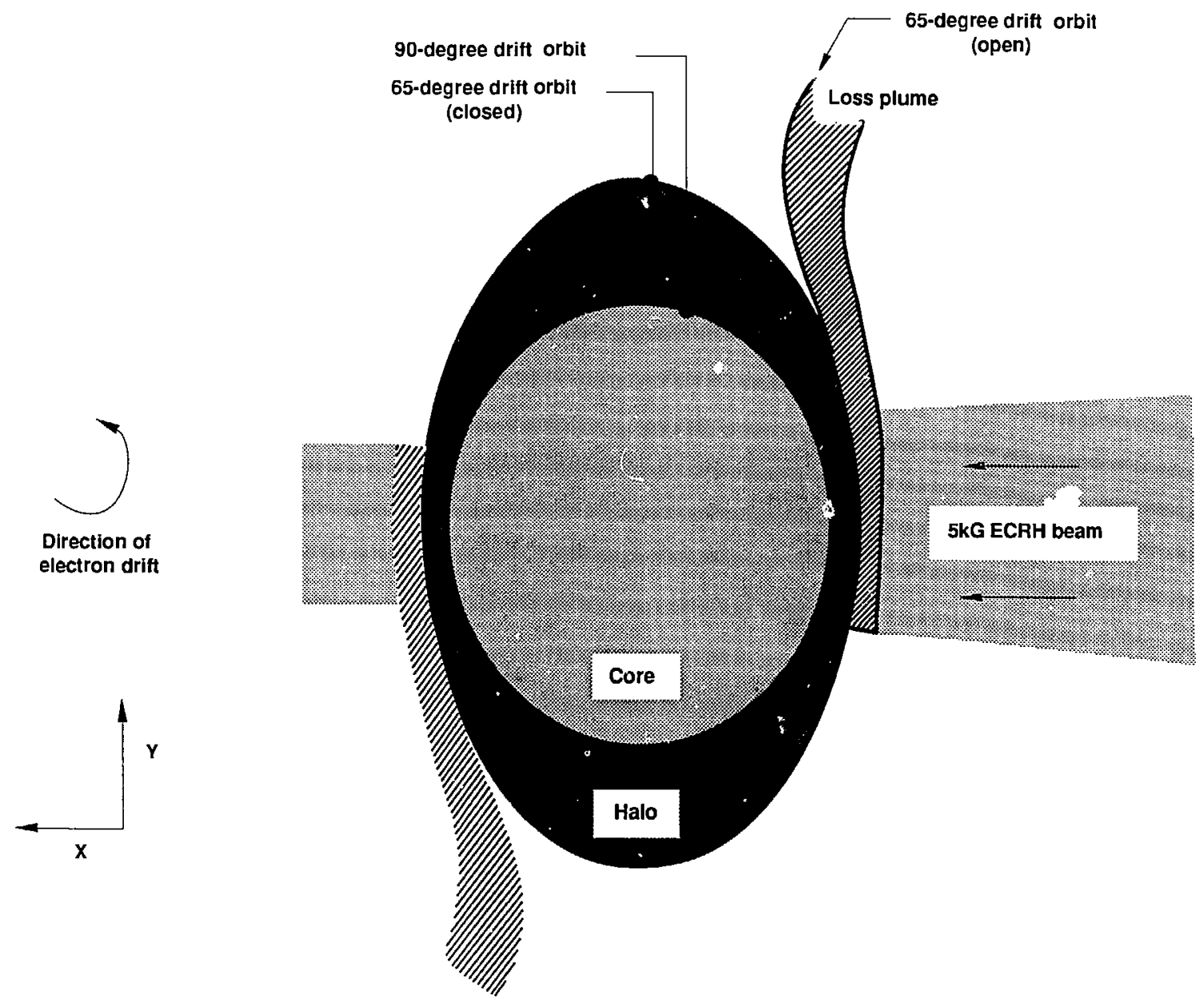

Figure 3.21. Determination of the plasma boundary by open drif surfaces in the West end-celi. 
component with a temperature in the range of 2-5 keV. In our experiments, the cold-component was dominant in the core, with an average density of $6 \times 10^{11}$ $\mathrm{cm}^{-3}$, while the warm-component density was $3.5 \times 10^{11} \mathrm{~cm}^{-3}$.

However, note that during standard thermal-barrier operation (specifically, operation jncluding sloshing-ion beams) at low center-cell densities, the end-cell coldcomponent density can become quite small, rcsulting in a large mirror-trapped fraction. Previous measurements of the hot-electron fraction that were based on $x$-ray measurements alone underestimate the mirror-confined fraction because they do not include the contribution of the warm-component core electrons ${ }^{12}$. During such operation, ion end-Ioss goes to zero ${ }^{22}$, and the axial potential profile shows the presence of the desired thermal-barrier and plug potentials ${ }^{23}$. Apparently, the barrier potential is created by the large fraction of 3-kr V varm, mirror-trapped elcctrons in the core region.

\subsection{Initial Hot-Electron Plasma Formation}

In TMX-U, hot-electron plasma forms near the magnetic axis, then expands within $10 \mathrm{~ms}$ to its equilibrium boundary. The measured expansion times are in good agreement with a model based on a parabolic plasma pressure profile. The experimental characterization of the hot-electron buildup was carried out in the West end-cell only, but we expect the process to be similar in both end-cells.

The variation of diamagnetism versus tine is shown in figure 3.22(a). Data from two shots have been overlaid; each shot has the limiter at a different radius, as indicated in the figıre. Each curve rises with the same slope for a period of time, then, after a time $\Delta t$, breaks over and rises more slowly. The delay time from the start of the buildup to this break is plotted as a function of limiter radius for five different probe scans in figures $3.23(\mathrm{a}-\mathrm{e})$. The data are fairly noisy but show a definite tendency to rise with increasing limiter radius.

In a similar fashion, overlays of time histories of the PECE hot-component line density are shown in figure $3.22(\mathrm{~b})$. The line density increases rapidly and saturates in less than $10 \mathrm{~ms}$ when the probe is withdrawn. When the probe is fully inserted, the buildup occurs more rapidly. 'The saturat iom of the line density is the physical mechanism behind the break in the diamagnetism described above. 
(a) Dlamagnetism

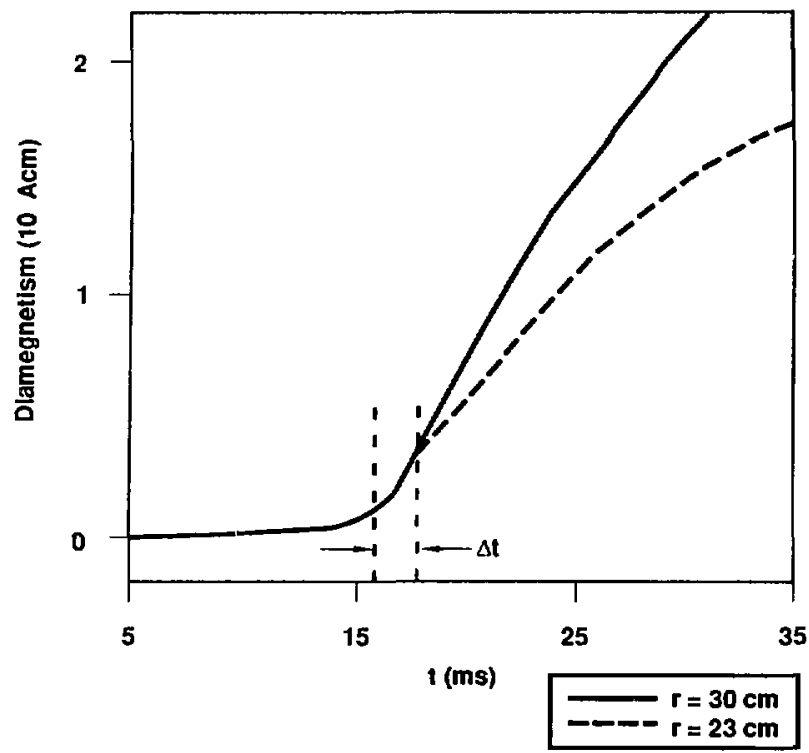

(b) Super-hot line density

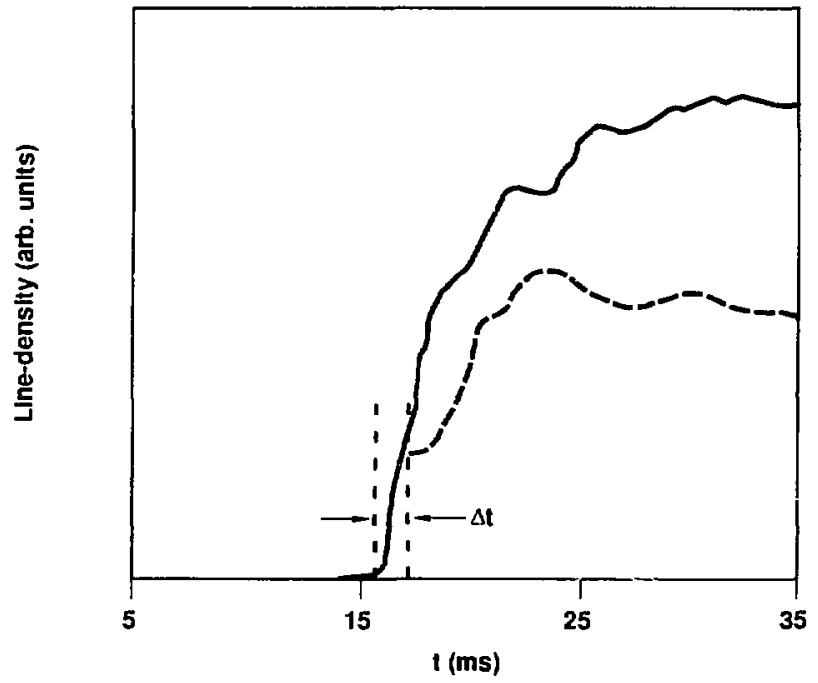

Figure 3.22. Time-history overlays of (a) diamagnetism, and (b) super-hot-component line density. 
The variation of the time delay to PECE line density saturation is also plotted in figure 3.23. These data are consistent with the DML break results as expected. The line drawn is interpolated with a cubic-spline method ${ }^{17}$ through the average value of all the data points, both from the DML and the PECE.

The PECE temperature time history does not change much with probe radius. The temperat ure climbs throughout the buildup and saturation of the line density. However, the temperature remains low during this period, less than $100 \mathrm{keV}$. An example of this behavior is shown in figure $4.9(\mathrm{~d})$.

Taken together, these measurements suggest a scenario for the initial hotelectron buildup. First, hot-electron plasina with a temperature of less than $100 \mathrm{keV}$ forms near the vacuum second-harmonic resonance. The increasing hot. clectron density at this location introduces a diamagnetic depression of the local field, and in the shallow radial well of the TMX-U magnetic field the secondharmonic resonance is forced outward. Eventually, the hot-electron line density saturates as the plasma expansion is "limited," either physically by one of the limiter probes or by other mechanisms that reduce confinement on the edge of the plasma (see section 4.4).

We can further analyze the temporal variation of the hot-clectron boundary. If we assume that the limiter first interarts with the plasma boundary after the time delay $\Delta t$, then the data presented determine the teinporal variation of the plasma boundary. The results are shown in figure 3.24 . The data points shown are interpolated from the PECE and DMI, time delay measurements while the curves are the best fit ellipses. The plasma initially expauds very rapidly, reaching its maximum cxtent after about $6 \mathrm{~ms}$. The plasma eccentricity remains nearly constant at 1.4 throughout its expansion.

The experimental results can be compared to two simple models for t'e expanding plasma. The simplest model is an expanding elliptical plasma with uniform pressure, $P_{\perp 0}$, and constant eccentricity, $c$. 'The stored energy per unit length, $W_{l}$, is the product of the cross-sectional area with the pressure, $W_{l}=\pi b^{2} P_{\perp 0} / c$. Early in time, the diamagnetisin has a constant slope, so the stored energy per unit length is $\dot{W}_{l,} t$. For this condition, the model gives the 
a) $\phi=90$ degrees

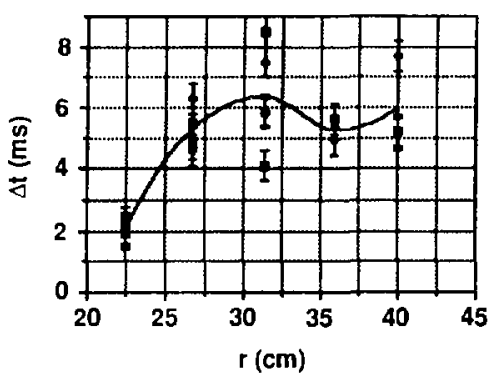

c) $\phi=60$ degrees

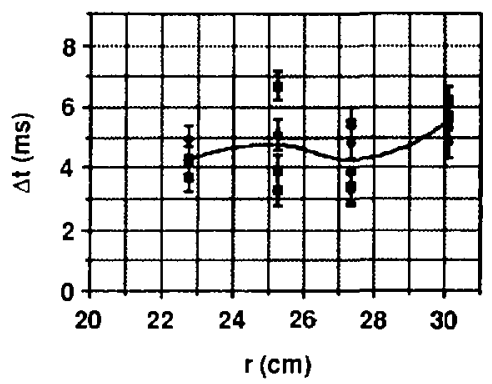

b) $\phi=70$ degrees

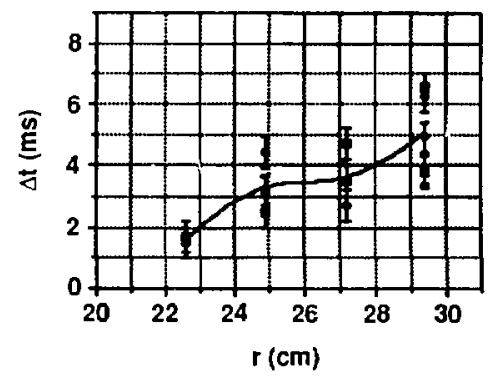

d) $\phi=\mathbf{5 0}$ degrees

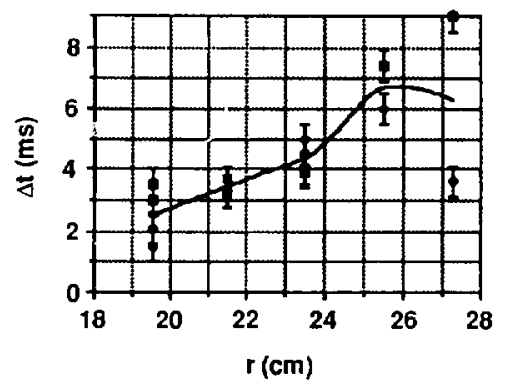

e) $\phi=0$ degrees

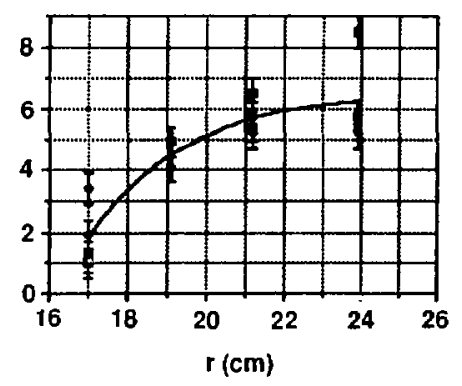

- PECE

- DML

Figure 3.23. Delay time to DMI break and PECE line density saturation versus liniter midplane radius. 


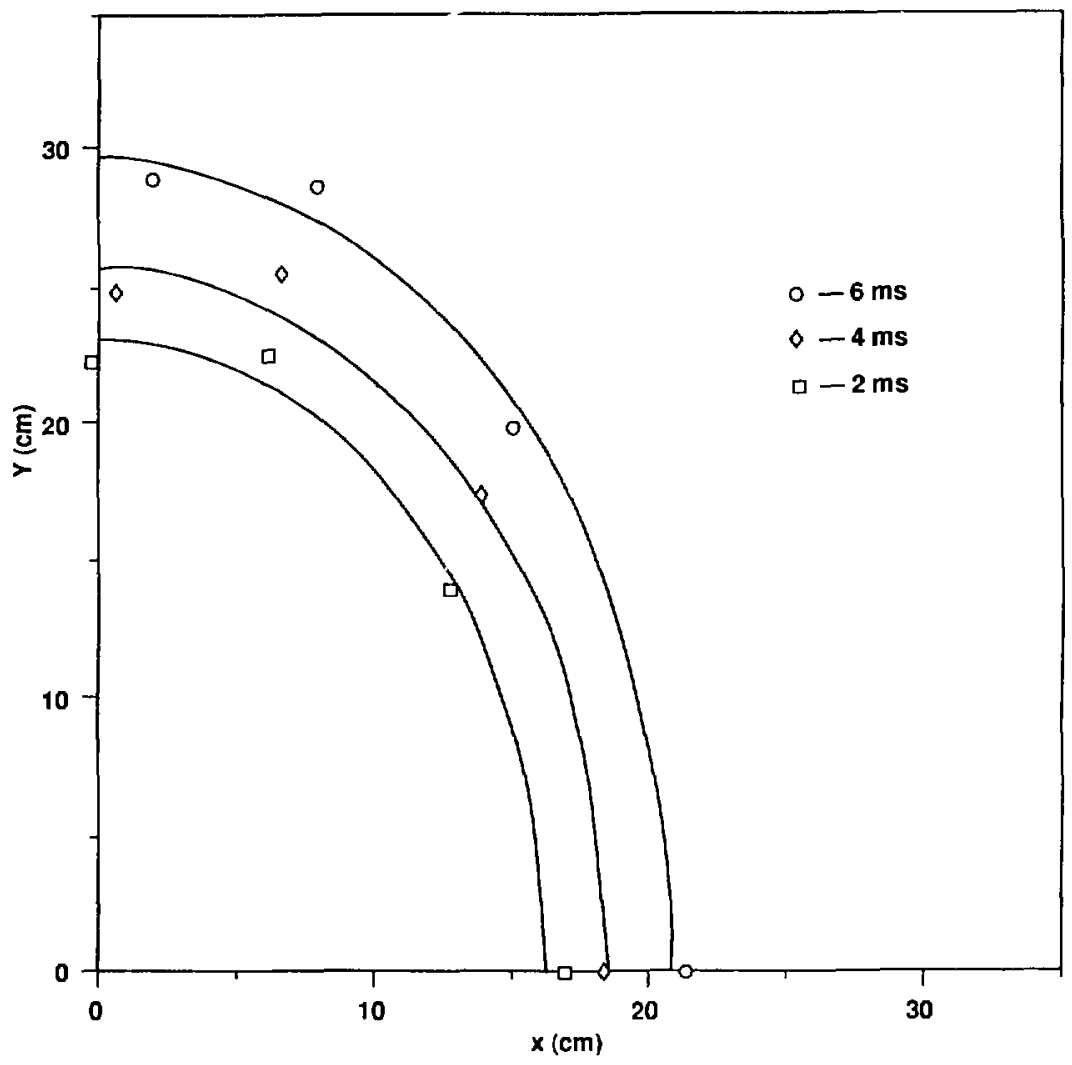

Figure 3.24. Temporal developuent of the hol-electron boundary. 
length of the semi-major ellipse bas a function of time:

$$
b: \sqrt{\frac{c \dot{W}_{L 0} t}{\pi \beta_{0}} \cdot \bar{P}_{0}^{-}}
$$

where $P_{0} \quad-B_{0}^{2} / 2 \mu_{0} ; B_{0}$ is the midplane magnetic field; and the perpendicular pressure of the plasma has been re-expressed in terms of the plasma beta, $P_{\perp_{0}}=$ $\beta_{0} P_{0}$.

A somewhat more complicated model is based on the assumption that the plasma pressure exactly "fills" the radial magnetic well to some depth,

$$
B(r) \sqrt{1-\beta(r)}=\eta B_{0},
$$

where $\eta$ is a free paraneter. The radial magnetic well in TMX-U is described approximately by a parabolic variation (see figure 3.26 ),

$$
B(r)=B_{0}\left(1+\delta \frac{r^{2}}{r_{0}^{2}}\right),
$$

where $:$ and $r_{0}$ are obtained from computer calculations of the TMX-U magneticfield profile and have the properties $\delta \ll 1, r / r_{0} \leq 1$. Define $\alpha \equiv 1-\eta \ll 1$, and expand $B(r)$ to second order in small quantities:

$$
\beta(r)=1 \cdot\left(\eta_{\frac{B_{0}}{B(r)}}\right)^{2}=2\left[\alpha+\delta\left(\begin{array}{c}
r \\
r_{0}
\end{array}\right)^{2}\right] .
$$

The diamagnetisin is proportional to the integral of $\beta(r)$. Carrying out the integration over an elliptical region with serni-major axis length $b$ and eccentricity $\epsilon$, we obtain

$$
W_{l}=P_{0} \frac{\pi b^{2}}{c}\left[2 \alpha+\left(1+\frac{\left.1 / \epsilon^{2}\right) \delta b^{2}}{2 r_{0}^{2}}\right] .\right.
$$

where $W_{l}$ is the stored energy. Note that this model simplifies to the previous one when $\delta=0$. The results of the two models are compared with the data in figure 3.25 . The free parameters of the models have been selected to provide the the best fit to the data at $\Delta t=2 \mathrm{~ms}$. For the uniform pressure models, the normalization yields $\beta_{0}=1.0 \%$, while for the parabolic model, $\beta_{0}=2 \alpha=0.5 \%$. The parabolic model gives a better fit. We expect the expansion to slow even further at late times as the plasma confinement deteriorates due to bad drift orbits; the limiting radius is determined by these open-drift surfaces. The relationship of plasma spatial structure to confinement is discussed further in chapter 4. 
a) $Y$-axis expansion

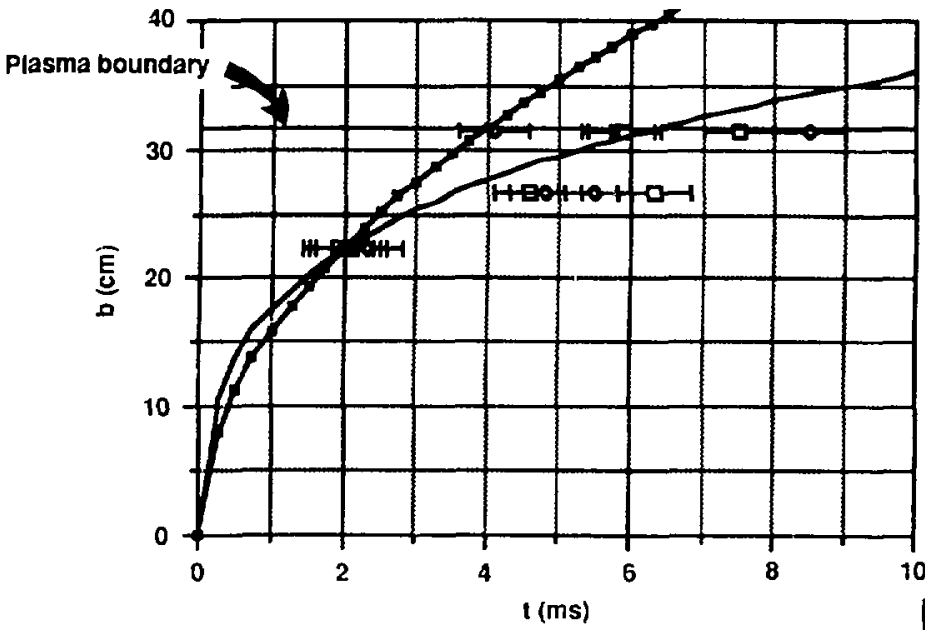

b) X-axis expansion

\begin{tabular}{|ll|}
\hline 口 & DML \\
- & PECE \\
- & Uniform \\
- & Parabolic \\
\hline
\end{tabular}

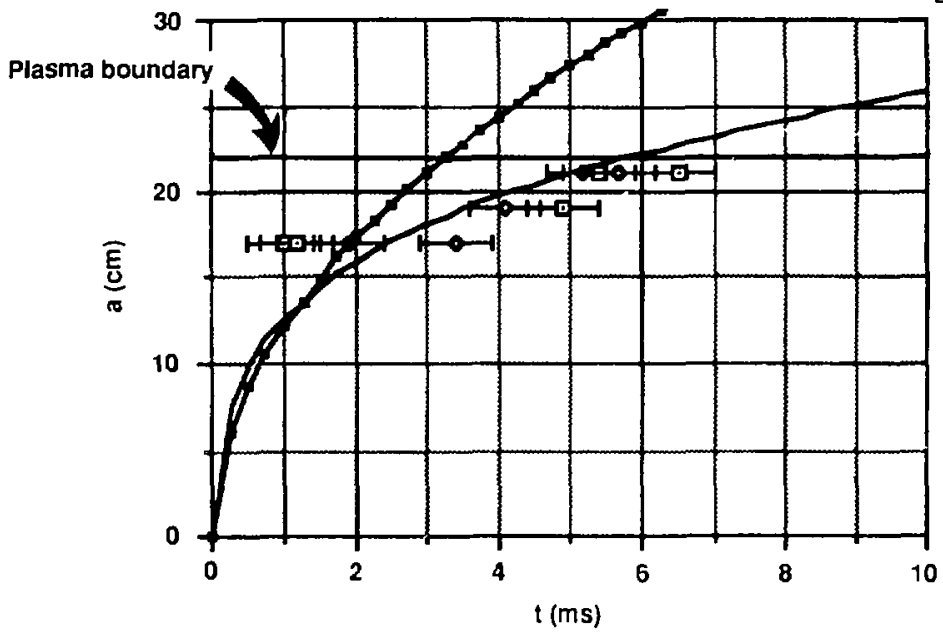

Figure 3.25. (emparison of the plasma cxpatnsion datia with two simple preasure mordels. 
(omparison of the theoretical models with the measured data indicates that the initjal plasma formation can be described in terms of a rigid expansion, with the pressure profile becoming hollow as the expansion proceeds. However, the rapid expansion also could be assisted by electron radial transport processes, as described in section 4.4.4 and appendix B. We cannot quantify the extent of this effect, however, because the plasma pressure profile is not known in detail during the early formation period.

\subsection{Effect of the Midplane Magnetic Field on Hot-Electron Plasma Formation}

The hot-clectron plasma formation is dramatically affected by the midplane vacuum magnetic-field offset. In this section we examine the variations in diamagnetism, PECE, hot-electron end-loss spectrurn, and total plasma line density. The results will be compared to the plasma structure and formation studies already presented. We begin with a description of the vacuum magnetic-field scan experiment.

\subsubsection{Vacuum Magnetic-Field Variation Experiment}

Data from the West end-cell are presented for the magnetic-field scan experiment of $10 / 30 / 86$. On this day the magnitude of the midplane magnetic field was varied from $-7 \%$ to $+4 \%$ relative to the standard TMX-U configuration. The field variation was accomplished by varying the coil currents in the end-cell solenoids and Joffe bars. The Ioffe bar currents generally did not follow the solenoid currents, so both the magnitude and shape of the rninimum-B well changed during the scan. This variation in the magnetic topology complicates the results; nonetheless, decreasing the coil currents below the standard settings has the general effect of moving the initial second-harmonic resonance outward in radius, nimicking the effects of the plasma's temporal increase in diarnagnetism. Furthermore, if the field is increased slightly above nominal (2\%), the second-harmonic resonance is eliminated entirely from the plasnia volume. 
Figures 3.26 (a) and (b) show the radial and axial magnetic-field profiles expressed in percent relative to $5 \mathrm{kG}$. The radial profile corresponds to $z=582 \mathrm{~cm}$, which is where the $5-\mathrm{kG}$ ECRH beam intersects the magnetic axis. The radial well is particularly shallow, with a shift of only $1.5 \%$ necessary to move the rescnance out to the worst case drift orbit separatrix.

Fueling conditions were held constant throughout the experiment. Deuterium gas flow was adjusted to $25 \mathrm{Tl} / \mathrm{s}$ duriug the first few discharges to give a line density of about $3 \times 10^{13} \mathrm{~cm}^{-2}$ in the West end-cell. Two different magnetic-field scans were carried out, each using a different ECRF heating waveform. One B-field scan was carried out with a constant amplitude $10-\mathrm{kG}$ system heating pulse, while the 5-kG system beating pulse was modulated at about $100 \mathrm{~Hz}$. Figures $3.27(\mathrm{a})$ and (b) show typical incident ECRH waveforms for this arrangement. The 10-kG system provided about $95 \mathrm{~kW}$, while the $5-\mathrm{kG}$ system provided about $120 \mathrm{~kW}$ at the peaks of the modulation; note that the $5-\mathrm{kG}$ system modulation is incomplete. The second $\mathrm{B}$-field scan was carried out with the reverse arrangement; the 5 - $\mathrm{kG}$ heater was operated "cw," and the 10-kG system was modulated. Typical heating waveforms for this mode of operation are shown in figures 3.27(c) and (d). Peak power levels vary depending on whether the gyrotron was modulated.

\subsubsection{Data}

The diamagnetism variation is shown in figure 3.28(a). The general behavior is similar in the two end-cells. At high field offset, the diamagnetism is very small. As the second-harmonic resonance is introduced, the diamagnetism rises sharply, reaching a maxinum at a field offset of about - $1 \%$. As the field is reduced further, the diamagnetism decreases more gradually.

The PECE results for the West snd-cell are shown in figures $3.28(\mathrm{~b})$ and (c). The super-hot component temperature is lower at the high ficld offsets, where the diamagnetism is also lower as mentioned. Then it increases sharply as the secondharmonic resonance is introduced. For both 5 - and 10 -kG ECRH modulation, the high-field-offset temperatures are low, about $150 \mathrm{keV}$. For the 5 -kG modulation, as the on-axis field drops below the resonance the temperature reaches a plateau at about $210 \mathrm{keV}$. The 10-kG FC, IRH modulation, however, sliows a more resonant 
(a) Transverse B-fleld variation

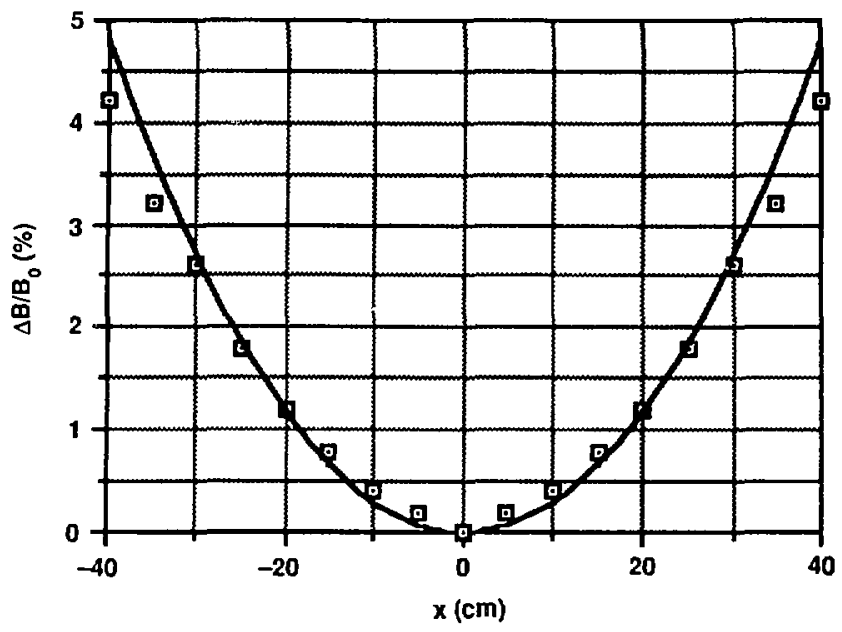

D Calculated B-tleld

- Parabollc fit

(b) Axial B-field variation

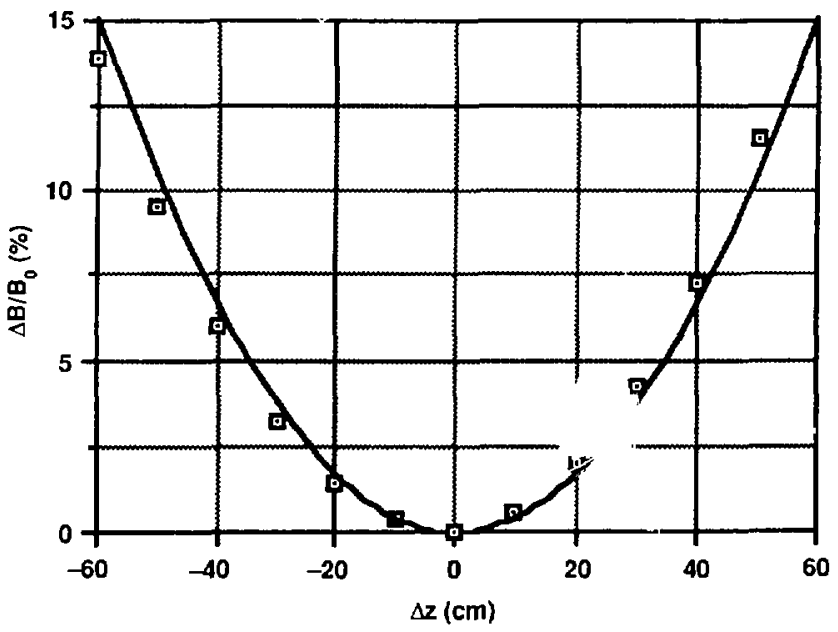

Figure 3.26. Magnetic field profiles: (a) $\mathrm{x}$-axis variation at 5-kG location; (b) axial variation at $r=0$. 
a) 'CW' 5-kg ECRH

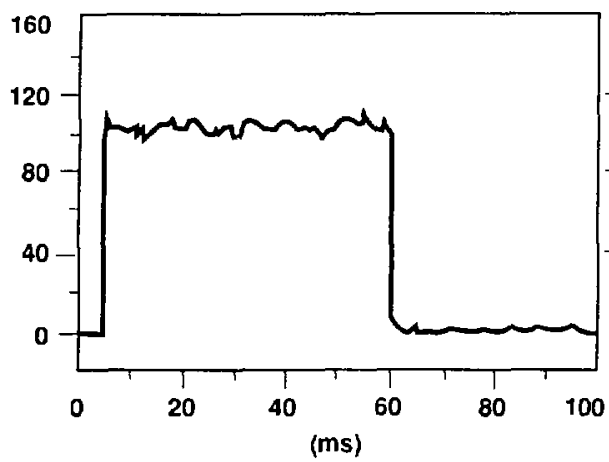

b) Modulated 10-kg ECRH

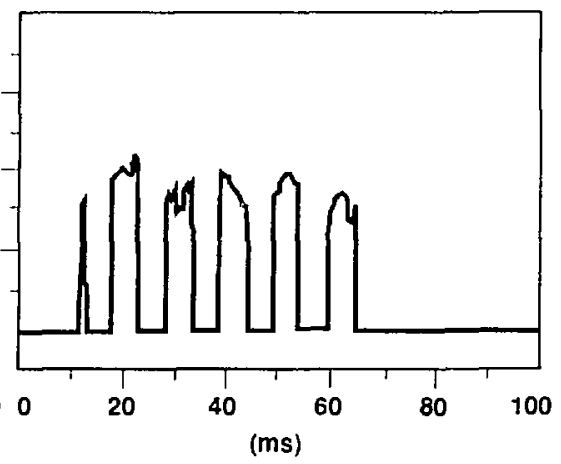

c) Modulated 5-kg ECRH

d)'CW' 10-kg ECRH

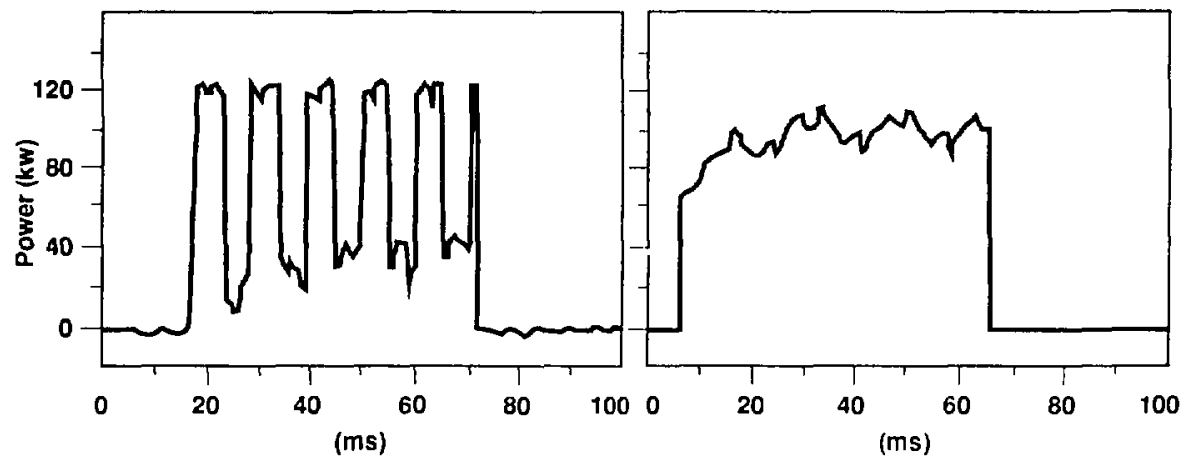

Figure 3.27. 'Typical EC'RH waveforms for the B-field scan experiment. 
behavior, with a peak temperature between 250 and $300 \mathrm{keV}$, then dropping for very low field offsets. The behavior of the peak super-hot line density is shown in figure 3.28(c). Like the diamagnetism, the line density is small at high field offset, rises to a peak as the second-harmonic resonance is introduced, then falls as the field decreases further. The peaking near resonance is sharper for the 5-kG ECRH modulation than for the 10-kG modulation.

By combining the diamagnetism data with the PECE temperature, we obtain an estimate of the super-hot-component column density:

$$
n_{A, \text { hot }} \equiv \int n_{\text {hot }} d A=\frac{\int d A, P_{\perp}}{<\sin \theta>T_{\text {hot }}} .
$$

Here, the cross-sectional average of the perpendicular pressure is given directly by the diamagnetism measurement, and we have again assumed a uniform temperature profile. The term $\langle\sin \theta>\approx 0.77$ accounts for the anisotropy as discussed previously. The results of this analysis are shown in figure $3.28(\mathrm{~d})$. The variation with $\frac{\Delta B}{B_{0}}$ is similar to that of the line density.

\subsubsection{Analysis}

Because all of ine hot-electron measurements obtained during the experiment show a maximum for a field offset of $\frac{\Delta B}{B_{0}} \approx-1$ to $-1.5 \%$, there appears to be a systematic error between the predicted and actual magnetic-field strengths. We will assume in subsequent aralysis that $\frac{\Delta B}{B_{0}}=-1.5 \%$ corresponds to the nominal field profile. If so, the second-harmonic resonance is moved from the magnetic axis to the drift-orbit separatrix $(|x| \approx 22 \mathrm{~cm})$ by shifting the field from $-1.5 \%$ to $-3.0 \%$.

The B-field scan data distinguishes the effects of fundamental and secondharmonic ECRF heating. Plainly, the hot-electron plasma does not buildup unless the second-harmonic resonance is present within the magnetic well. Therefore, in TMX-U, heating at the fundamental resonance does not produce mirror-trapped hot electrons. This observation is consistent with other experiments where hotelectron build up proceeded normally with only the 5-kG heater in operation, but fails when the 10-kG system is operated independently. The lack of effectiveness of fundamental heating is in profound disagreement with previous Fokker-Planck 
(a) Dlamagnetism

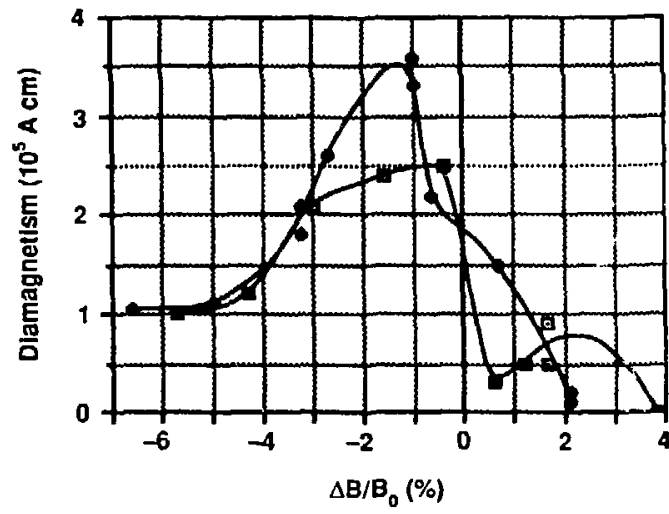

(c) Relative super-hot-electron line-density

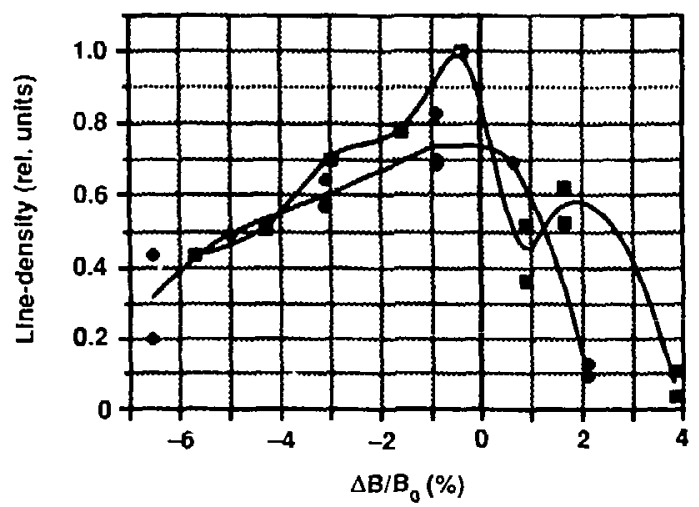

(b) Temperature

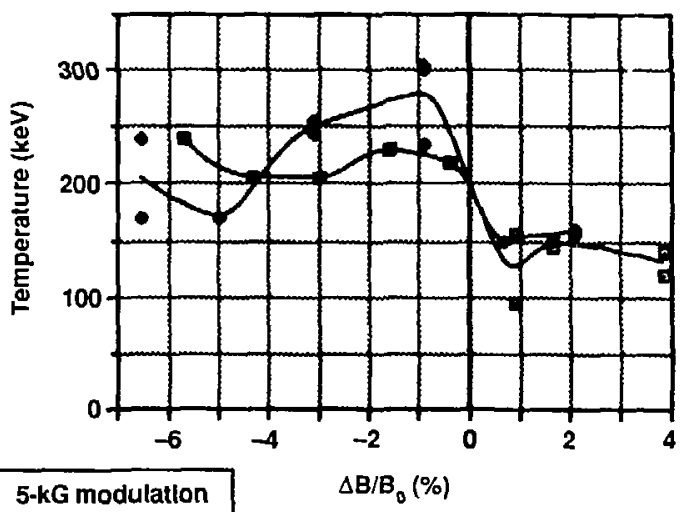

(d) Column density

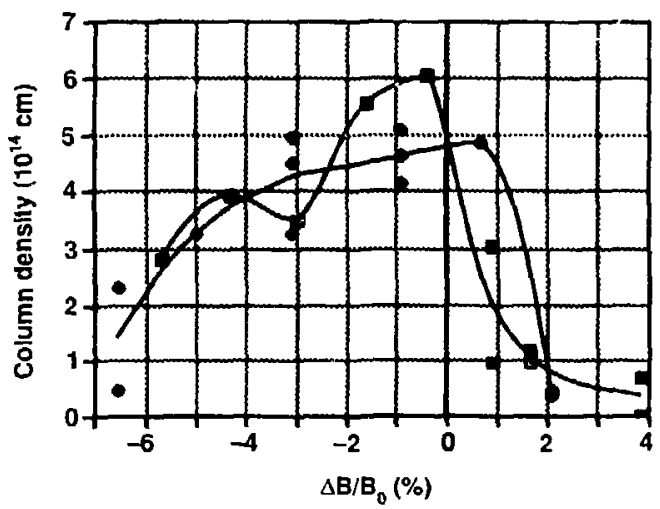

Figure 3.28. B-field-scan experiment super-hot-component data: (a) diamagnetism, (b) temperature, (c) line density, (d) column density. 
simulations ${ }^{24}$. Therefore, second-harmonic ECRH dominates the hot-electron plasma formation process.

The supcr-hot component plasma can be modeled as consisting of two regions of uriform density and temperature, as suggested by the previous measurements of hot-electron plasma siructure. We term the halo density $n_{2}$, and the core density $n_{1}$, and assume that the temperature is constant throughout both regions. We can easily relate the model parameters to the measured quantities:

$$
n_{A}=\int n d A=\left(A_{2}-A_{1}\right) n_{2}+A_{1} n_{1},
$$

and

$$
n_{l}=\int n d y=2\left(b_{2}-b_{1}\right) n_{2}+2 b_{1} n_{1} .
$$

where $n_{A}$ is the column density previously described; $n_{l}$ is the line density; $A_{1}$ and $A_{2}$ are the areas corresponding to the inner and outer ellipses, respectively; and $b_{1}$ and $b_{2}$ are the lengths of their semi-major axes. The equations are easily solved to obtain $n_{1}$ and $n_{2}$ :

$$
n_{2}=3.43 n_{c}-0.97 n_{l} ; \quad n_{1}=0.66 n_{l}-1.52 n_{c} .
$$

Here, $n_{l}$ is in units of $10^{12} \mathrm{~cm}^{-2} ; n_{A}$ is in units of $10^{14} \mathrm{~cm}^{-1}$; and $n_{2}$, and $n_{1}$ are jrl units of $10^{11} \mathrm{~cm}^{-3}$. The "hollowness" of the profile is the ratio

$$
h=\frac{n_{2}}{n_{1}}=2.26 \frac{k-0.28}{0.43-k},
$$

where $k:=n_{c} / n_{l}$ expressed in the above units. The two-region model is valid where $0.28 \leq k \leq 0.43$. If $k<0.28$, the halo is empty, $n_{2}=0$, and we use a single-region, uniform cylindrical plasma model with variable outer radius, $r_{1}$. For this case,

$$
r_{1}=100 \frac{2}{\pi} k ; \quad n_{1}=10 \frac{n_{l}}{2 r_{1}} .
$$

Finally, when $k>0.43$, the core becomes empty, $n_{1}=0$, and the plasma in fully annular. For this case we assume that the annulus has fixed dimensions and obtain the density from:

$$
n_{2}=0.5\left(1000 \frac{n_{\mathrm{c}}}{A_{2}-A_{1}}+10 \frac{n_{1}}{2\left(b_{2}-b_{1}\right)}\right) .
$$

To apply this model, we need an absolute measurement of the super-hot component line density. In the West end-cell, we can calibrate the PECD measurement 
from the previous measurement of the plasma structure in section 3.1. The azimuthally averaged pressure profile can be converted to density and drift-mapped back to $90^{\circ}$ essentially the reverse of the previously described procedure. The line density obtained from this process is $1.4 \times 10^{13} \mathrm{~cm}^{-2}$. For the limiter probe scan data of the previous sections, the field was slightly depressed, $\frac{\Delta B}{B_{0}} \approx-0.8 \%$, and the data is normalized accordingly.

Applying the model to the data produces the densities shown in figure 3.29. For very high field offsets, the hot-electron plasma is only in the core region. As the second-harmonic resonance is introduced, the plasma immediately becomes hollow. When the field is reduced further, the plasma becomes completely annular and the density decreases. At extremely low field offset, the plasma again appears in the core region.

Thus, the West end-cell B-field scan results are consistent with the previous measurements of plasma structure and formation. The super-hot component plasma forms at the initial second-harmonic resonance and spreads outward. It is particularly interesting to note that, at a small positive field offset, a relatively dense plasma is formed in a narrow column $(r \approx 5-10 \mathrm{~cm})$ near the magnetic axis. When the vacuum second-harmonic resonance is moved off the magnetic axis, the plasma structure becomes annular. As the resonance location is moved beyond the drift-orbit separatrix, the super-hot component density drops due to the reduced confinement. Plasma formed ouiside the worst-case separatrix can drift on closed surfaces for a smaller class of pitch-angle, so the plasma is lost radially more rapidly. This effect is verified by HEED probe measurements of the radial loss power (see section 4.4). Finally, for the lowest magnetic-field settings, the resonance is removed entirely from the second-harmonic beamline. Nonetheless, a hot-electron population still builds up. This indicates that the hot-electron plasma can build up entirely under the influence of cavity heating. In this case, the plasma density is lower and more spatially uniform. 


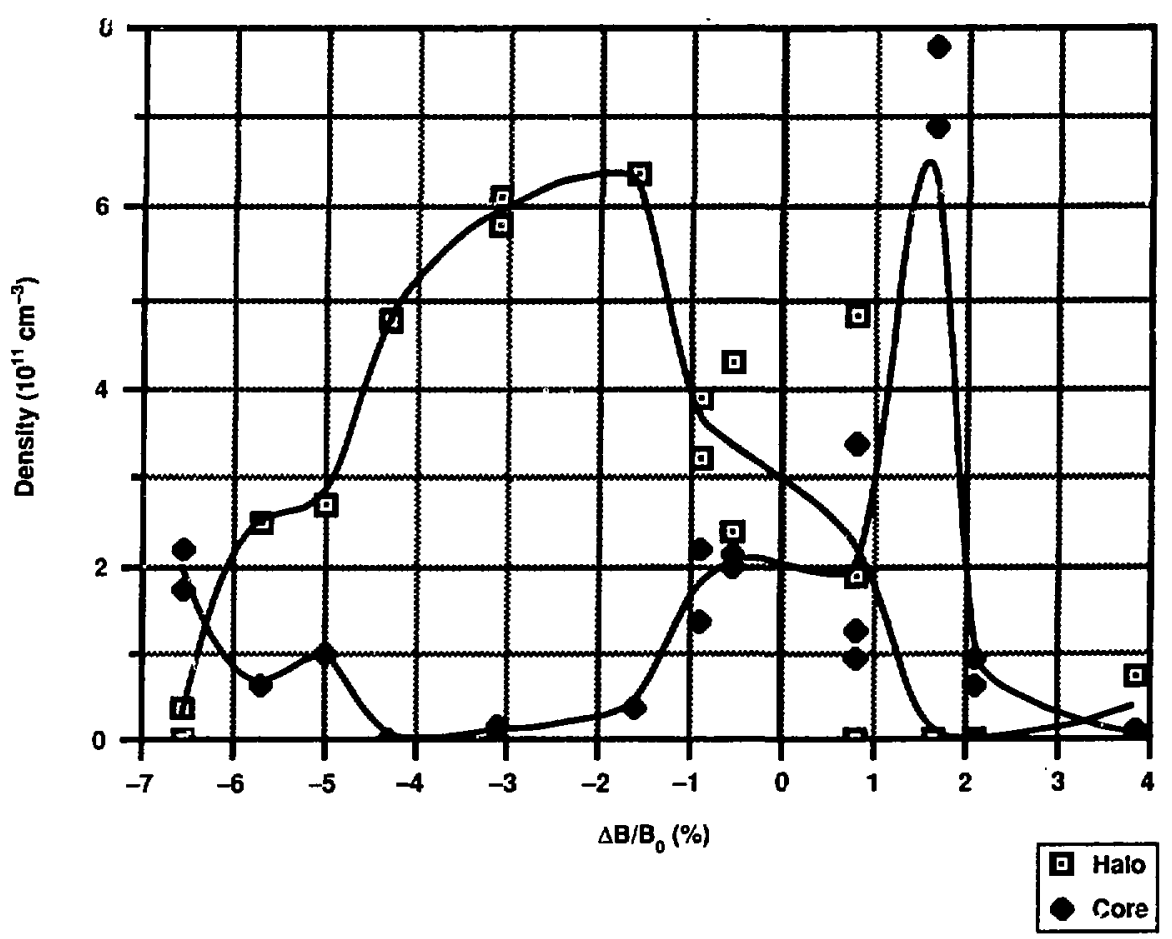

Figure 3.29. West end-cell plasma structure variation with $\frac{\Delta B}{B_{0}}$. 


\section{Chapter 4}

\section{Hot-Electron Plasma}

\section{Confinement}

This chapter is concerned with the confinement of energy by the hot-electron Fiasma. We begin by examining ECRF absorption. Global absorption (the tota! power absorbed by the plasma from both ECRH systems) is fairly high, 50$70 \%$, throughout the shot, but drops somewhat as the discharge progresses. The experimental measurements are a good match to theoretical predictions of the second-harmonic and fundamental ECRF absorption. The majority of the power is absorbed in the vicinity of the beta-shifted second-harmonic resonance, while only a small fraction is absorbed at the fundamental. The power deposition code MECH is used to determine the first-pass absorption of ECRF rays through a plasma with the spatial structure described in the chapter 3 , and a simple model describes the cavity heating process through which a large fraction of the ECRH power can be absorbed.

Next is a study of energy storage by the hot-electron plasma. Energy is predominantly stored in the long-lived super-hot-component electrons. The confined power fraction, defined as the time rate of change of the stored energy, is initially $40-50 \%$ of the total incident ECRF power, but drops steadily as the discharge progresses. The decreasing super-hot-electron absorption is initially caused by the increasing temperature and diamagnetism of the super-hot electrons, while later in the discharge more power is deposited in the poorly confined hot-component plasma, leaving less power available for absorption by the super-hot-component electrons.

In the core region, axial electron loss is the dominant energy loss process. Early in the discharge, during the rapid hot-electron radial growth, the end-loss 
power flux is large and dominated by electrons with a temperature of $10 \mathrm{keV}$. As the resonance moves away from the $z$ axis, the end-loss power flux drops because of the decreasing average energy of the loss electrons decreases. Thereafter, the axial power-loss fraction is small, about $15 \%$, which is consistent with the weak ECRF absorption at the fundamental. The measured loss rate is consistent with collisional pitch-angle diffusion into the loss cone.

Radial loss onto open drift surfaces is dominaitt in the halo. It begins promptly with the onset of strong ECRH absorption, continues to rise as the plasma expands, then saturates. Limiter probe measurements show the spatial structure of an electron-loss plume that corresponds well to the open $65^{\circ}$ curvature drift surfaces. The measured radial power-loss fraction is typically $15-20 \%$, which is a good match to estimates of first-pass and cavity ECRF absorption by the hotcomponent at the second-harmonic resonance. Electron confinement in the hale is poor, much less than the $90^{\circ}$ pitch-angle scatter time, due to nonresonant neoclassical radial transport onto the open magnetic-curvature drift surfaces. Estimates of the energy-confinement time are made by combining Fokker-Planck simulations of the drift-loss mechanism with calculations of the radial transport rate. The results are in good agreement with the measured confinement times.

The study of confinement will focus on the West end-cell and use East end-cell data only as a complement. For the experimental characterization, we use the results of the vacuum magnetic-field scan to discern the effects of fundamental and second-harmonic heating, while the ECRH pulse modulation separates the effects of the two heating systems. This is necessary because cavity heating is important, and both 5-and 10-kG heating systems can provide power for cavity heating of the plasma at the second-harmonic. The limiter probe scans, described in detail in chapter 3 , are also used to provide spatial information about power absorption and loss in the West end-cell halo.

\subsection{ECRF Absorption}

This section discusses the absorption of ECRF power by the end-cell plasma. First, we present the microwave diagnostics used to measure the global and first-pass 5-kG ECRF absorption. Next, the absorption data is shown. Finally, the resuits 
are compared with theoretical predictions obtained from ray-tracing calculations and a simple cavity-heating model.

\subsubsection{ECRF Absorption Diagnostics}

\section{Global Absorption}

Global ECRH absorption is determined with an array of cavity probes. The probes sample the cavity electric field at several locations within the end-cells. An incoherent arerage of the electric field measurements provides a measure of the average electric field that is relatively insensitive to cavity-mode structure that can strongly modulate the field sensed by any one probe. The change in the average cavity electric field produced by the plasma determines the global absorption.

Two arrays of cavity probes measure the average eleciric field within each of the two end-cells. In the West end-cell there are eight probes, six of which are located at various locations near of the 5 - $\mathrm{kG}$ heater, while two are located near the $10-\mathrm{kG}$ heater. Five probes are located in the East end-cell, with three near the 5-kG heater and two near the $10-\mathrm{kG}$ heater. All probes are positioned to avoid the main beams of the ECRF heating structures.

Two types of antennas are used as cavity probes. The simplest consist of openended WR-42 waveguide. Three of the probes in the West end-ceil are of this type. Also used in the West end-cell are five slotted waveguide arrays, where the slots are spaced randomly along a length of waveguide to produce an omni-directional receiving pattern.

As both of the antennas are fairly uniformly illuminated from all directions, they have the same effective aperture, $A_{\text {eff }}=\lambda^{2} / 4 \pi$. However, the slotted waveguide arrays have a radiation efficiency, $\epsilon<1$. The radiation efficiency was determined by comparing the spatial average of measurements made in a 200-liter, cylindrical cavity with both slotted waveguide and op ended waveguide antennas. The measured value compares well to calculations based on the effective impedance of the slots.

Each antenna feeds a length of WR-42 waveguide, which is terminated by an attenuator and a waveguide diode detector. In general, the voltage across the 
diode detector has a nonlinear dependence upon the incident microwave power. It is common to operate such detectors at low incident power levels $(<100 \mu \mathrm{W})$ in the "square-law" region, where their response is approximately linear. However, in this region the detector response is a strong function of ambient temperature, due to the rapid variation of the diode reverse saturation current. Therefore, we chose to operate the diode at much larger incident power levels (i.e., as a peak detector). In this case, there is little sensitivity to temperature, although the relationship between voltage and power is no longer linear.

The detector voltage is buffered by an amplifier and low-pass filtered to avoid aliasing. The signal is then digitized by a CAMAC data logger and acquired into the TMX-U data base. A software routine converts the voltage signals into power levels. The conversion is accomplished using calibration data for each diode entered in the form of a look-up table.

Measurement of the average cavity electric field is complicated by high-order mode structure. In each end-cell the plasma is enclosed by an aluminum sheet metal structure called the "warm wall." The cavity formed by this wall is roughly cylindrical, with an average radius of $90 \mathrm{~cm}$, length of $300 \mathrm{~cm}$, and volume of approximately 00 liters. During a plasma discharge, more than $200 \mathrm{~kW}$ of monochromatic microwave power is injected into this structure, and it forms an overmoded cavity that traps the electromagnetic energy. The standing-wave patterns within the cavity are the source of large variations in the local, time-averaged electric field with a spatial scale length on the order of half a wavelength, $0.5 \mathrm{~cm}$. This mode structure is not stationary, but varies unpredictably due to phase variations in the gyrotron and rapid variations in the dielectric structure of the plasma.

Because each cavity probe is sensitive to both the magnitude and phase of the electric field within its aperture, their signals are strongly modulated by the cavity mode structure. The effect of such modulation is reduced by averaging over the entire array of probes, which tends to remove the coherent structure. It is convenient to express the average in the form of the average aperture Poynting flux:

$$
<S_{a p}>\equiv \frac{4 \pi}{\lambda^{2}}\left(\sum_{i}^{x-\text { mode }} \frac{P_{x, i}}{\epsilon_{i}}+\sum_{i}^{o-\text { mode }} \frac{P_{o, i}}{\epsilon_{i}}\right),
$$

where $\epsilon_{i}$ is the radiation efficiency of the $i^{i h}$ cavity probe. Further improvement is 
obtained by temporal smoothing, as most of the phase modulation introduced by plasma effects varies on a fast time scale; for this reason the cavity probe signals are low-pass filtered digitally to a 1-kHz bandwidth. Despite these procedures, the final average cavity field measurement is subject to noise introduced by residual modulation. The noise level is estimated to be less than $15 \%$.

The ECRF power absorbed by the plasma, $P_{a b a}$, is estimated from a simple electromagnetic power balance:

$$
P_{0}=<S_{a p}>A_{\text {lose }}+P_{a b o s},
$$

where $P_{0}$ is the total incident ECRF power, and $A_{a p}$ is the effective area of lossy apertures within the microwave cavity. $A_{\text {los }}$ is determined experimentally by firing the ECRH heaters without generating plasma, so that $P_{a b}$ is very small. This measurement gives an area of $0.65 \mathrm{~m}^{2}$ in the West end-cell, compared to the total cavity surface area of about $20 \mathrm{~m}^{2}$. The measured value is in agreement with estimates of the area of actual holes within the warm wall.

\section{ECRF Transmission Array}

A device to measure the spatial profile of the transmitted second-harmonic ECRH beam is located in the East end-cell. This transmission diagnostic consists of seven power samplers arrayed on a metal plate, as shown in figure 4.1. The central detector of the array is approximately aligned with the unperturbed location of the second-harmonic beam. Each power sampler is simply a $0.43-\mathrm{cm}$ hole feeding a length of waveguide terminated with a variable amount of attenuation and a waveguide diode detector.

The diode voltages are buffered, acquired, and converted to power levels, as described for the cavity probe signals. A weighted sum of the power signals from the seven channels provides an estimate of the total first-pass transmitted power, which is subtracted from the incident $5-\mathrm{kG}$ power level to determine the first-pass absorption.

\subsubsection{Temporal Behavior}

The temporal behavior of the absorption in the typical hot-electron discharge verifies the rapid radial growth of the plasma. Figure $4.2(\mathrm{c})$ is a typical time 

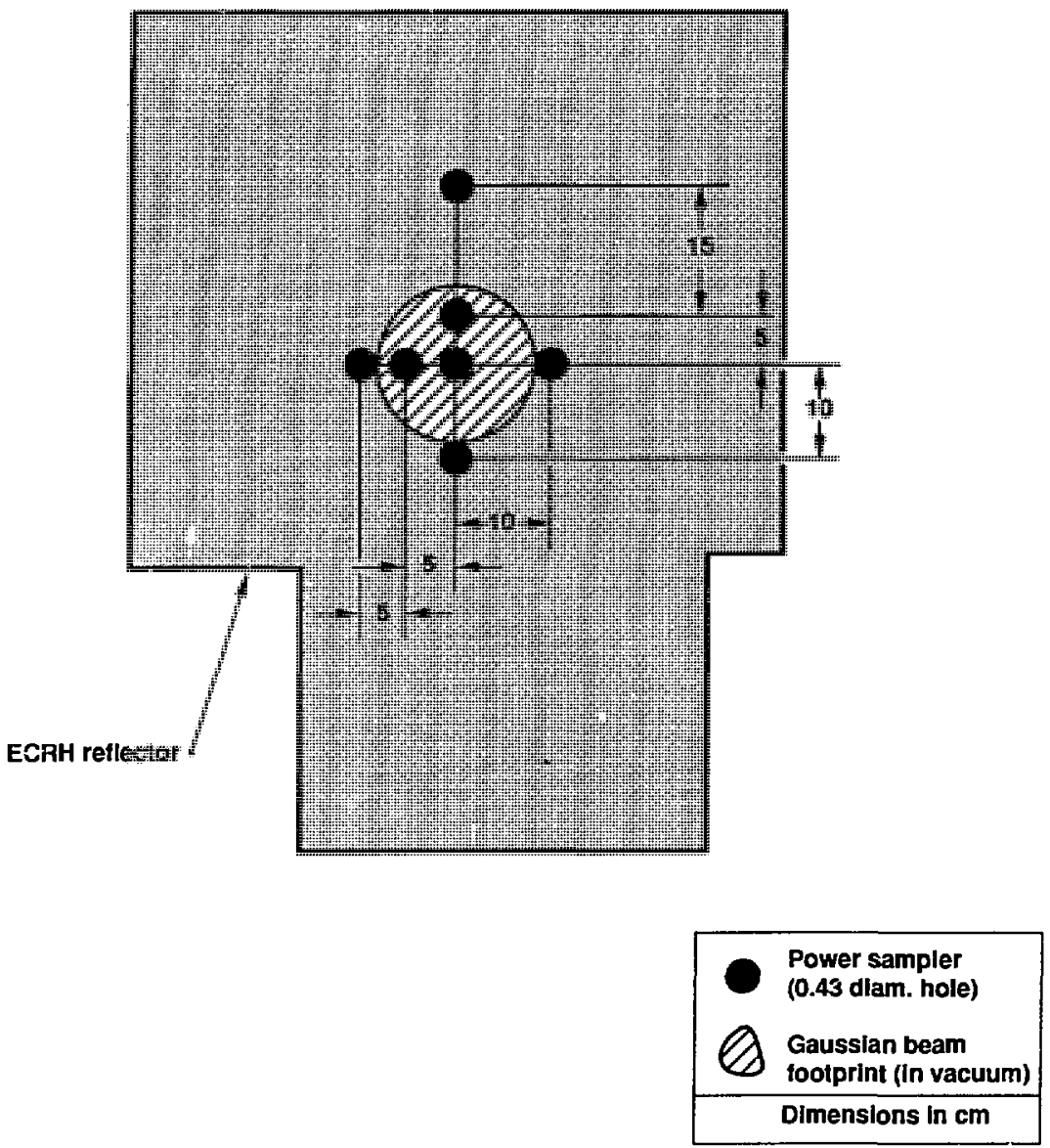

Figure 4.1. The first-pass 5-kG ECRH transmission array. 
history of the global ECRH absorption in the West end-cell, while figures 4.2(a) and (b) show the incident ECRH power waveforms. During the first pulse of the $10-\mathrm{kG}$ heater, absorption is low, less than $20 \%$ of incident. The absorbed power begins to rise concurrently wit3 the diamagnetism, reaching a peak of $70-90 \%$ of incident in 5-15 ms. Thus, the absorption increases as the plasma expands due to the increasing optical depth and absorbing surface area of the plasma; then it saturates as the plasma expansion is halted. For this particular discharge the absorption peaks within $5 \mathrm{~ms}$. Thereafter, by examining the modulation, we see that the 5-kG ECRH absorption slowly drops, while the $10-k G$ absorption remains nearly constant.

\subsubsection{Spatial Structure}

The global absorption of ECRH power in the West end-cell was measured during the four HEED probe limiter scans (see figures 4.3). The average drop is about $40 \%$ of the $5-\mathrm{kG}$ ECRF absorption as the probe is fully inserted, while the total absorption shows less effect. In absolute numbers, the average drop in absorbed power is about $30 \mathrm{~kW}$ regardless of modulation.

These measurements are consistent with the previously described halo structure. As the HEED probe is inserted, some of the electrons drifting from the beta-shifted second-harmonic resonance are scraped off. The process is analogous to moving the worst-case drift-orbit separatrix to larger pitch angle. As the probe is inserted halo confinement deteriorates, and the halo builds up to a lower density, thus trapping a smaller amount of ECRF power. The modulation reveals that the 5-kG ECRH is the primary driver of the halo plasma.

\subsubsection{Variation with Vacuum Magnetic Field}

We turn now to the absorption results from the B-field scan. The West end-cell results are shown in figure 4.4 . Generally, the absorption is small, around $10-20 \%$ of incident at high field offsets, and there is no apparent buildup of hot electrons. As the field is reduced below $+2 \%$, the larger average absorption of about $40 \%$ is caused by a partial buildup of the hot-electron plasma late in the ECRH pulse. For $\frac{\Delta B}{B_{0}}<0 \%$, the hot-electron buildup is prompt, and the absorption efficiency 
(a) 5 kG Power

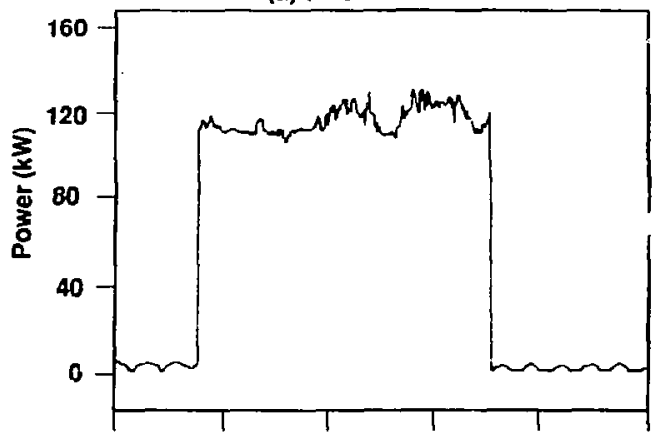

(b) iakG Power

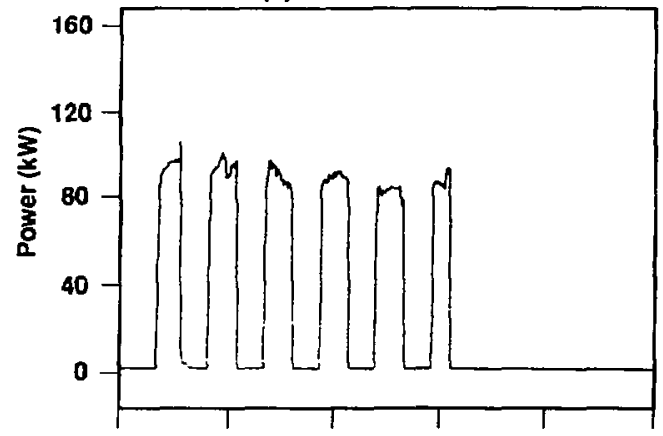

(c) Aborbed Power

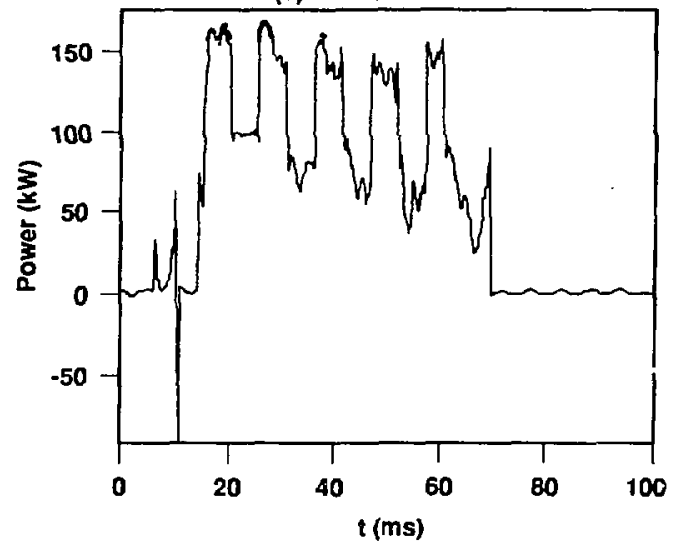

Figure 4.2. Time history of ECRF absorption in the West end cell: (a) 5-kG ECRH, (b) 10-kG ECRH, (c) global ECRF absorption. 
a) $\phi=\mathbf{9 0}$ degrees

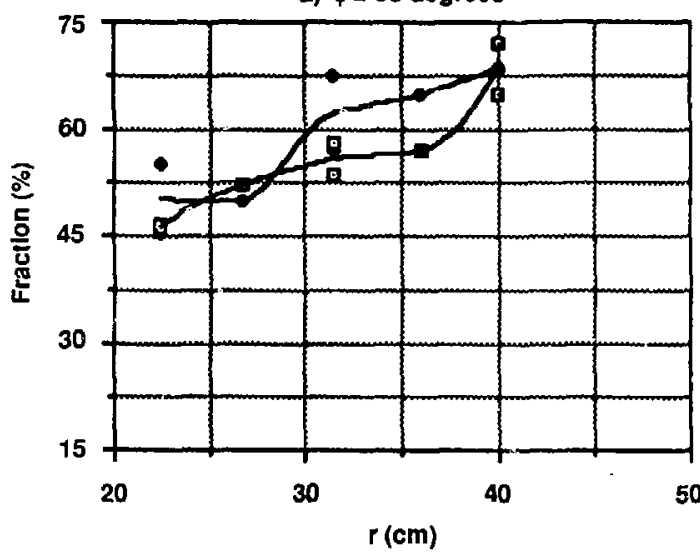

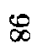

c) $\phi=60$ degrees

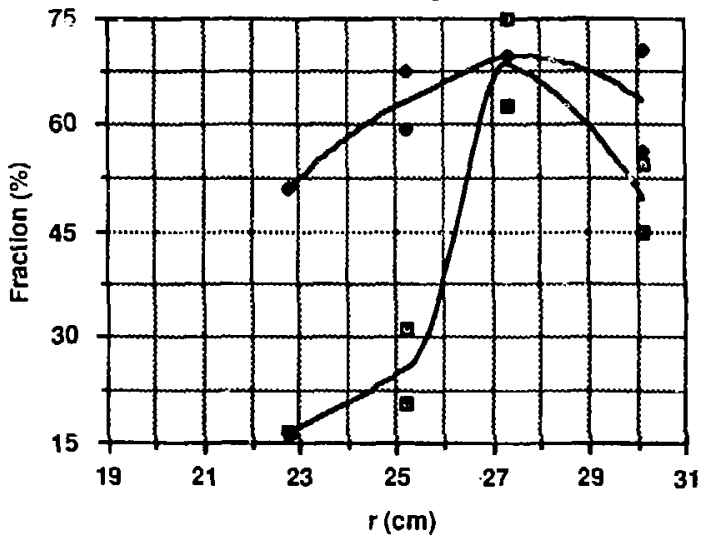

b) $\phi=70$ degrees

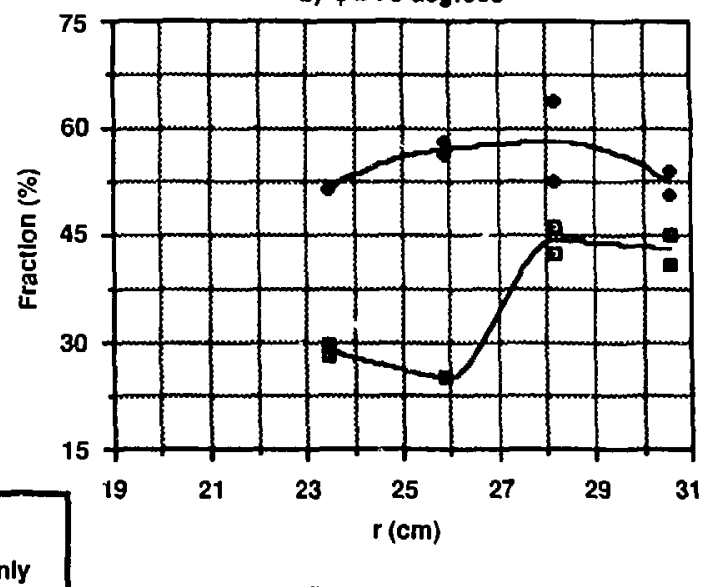

d) $\phi=50$ degrees

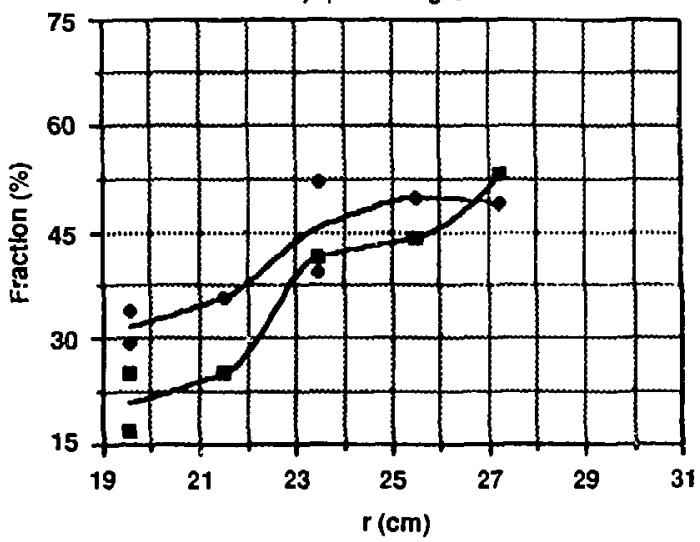

Figure 4.3. ECRH absorption versus limiter midplane radius for four limiter scans. 
is in the range of $50-70 \%$ with little variation with decreasing magnetic field. Peak absorption occurs near $\frac{\Delta B}{\bar{B}_{0}} \approx 1.5 \%$, which is believed to correspond io the nominal field configuration in which the second-harmonic resonance intersects the 5-kG beam near the $\mathrm{z}$ axis of the machine. The absorption efficiencies of the two ECRH systems are similar.

For comparison, the East end-cell absorption data are plotted versus $\frac{\Delta B}{B_{0}}$ in figure 4.4(b). The behavior is generally quite similar, although the absorption rises more sharply for $\frac{\Delta B}{B_{0}}<0 \%$ due to the lack of late-time buildup for slightly elevated field offset. In this end-cell, we measured the first-pass 5-kG absorption, see figure 4.4(c). Unlike the global absorption, the first-pass absorption has $\approx$ resonant character, with a peak occurring :t, $\frac{\Delta B}{B_{0}} \approx-1.5 \%$.

Two major conclusions are apparent from the measurements. First, the poor global absorption of the 10-kG ECRH when the second-harmonic resonance is not present indicates that fundamental heating is weak. First-pass absorftion of the fundamental is expected to be small, and cavity heating is not effective at the fundamental. Second, the resonant behavior of the first-pass 5-kG absorption demonstrates the significant role of cavity heating for very negative magnetic-field of set, or, equivalently, during the latter (equilibrium) phase of a normal discharge. Thus, the strong enhancement in 10-kG absorption efficiency subsequent to hotelectron buildup is due to cavity heating along the large second-harmonic surface areas of the fully expanded, plasma. A model for the cavity heating is presented in section 4.1.5.

\subsubsection{Comparison with Theory}

In this section, we compare the measured absorption with the predictions of the MECH code and a cavily heating model. The MECH code performs ray-tracing and power deposition calculations for a relativistic Maxwellian plasma and includes the effects of the equilibrium magnetic-field depression produced by the plasma (see appendix A). Ray tracing is performed for the $5-\mathrm{kG}$ heater with two spatial structure models that correspond to nominal and reduced midplane magnetic field conditions. Ray tracing is also carried out for the uniform heater. Next, we present a model for the cavity heating process and show that cavity absorp- 
(a) West end-cell gabal absorption

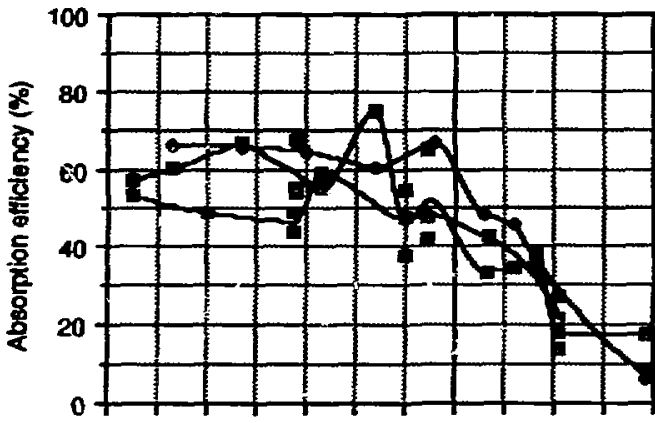

(b) East end-cell global absorption
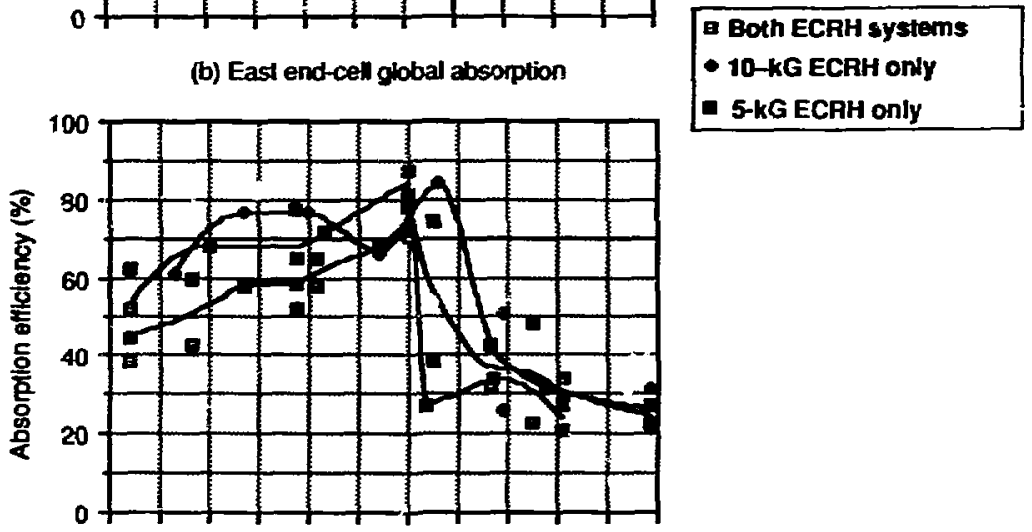

(c) East end-cell first-pass absorption

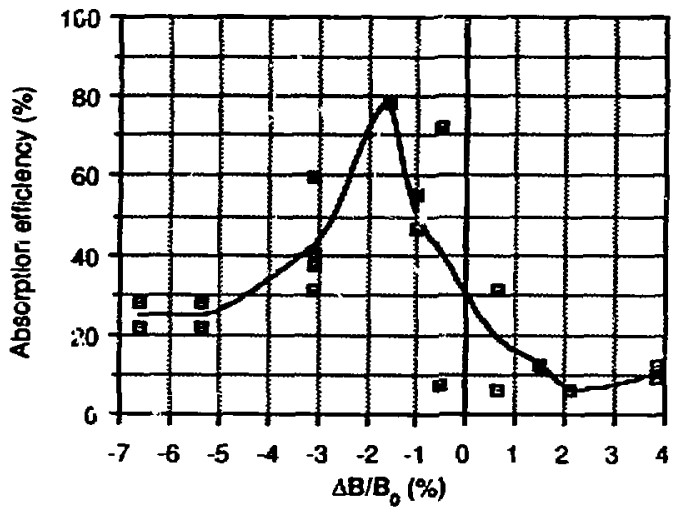

Figure 4.4. ECRF absorption efficiency versus $\frac{\Delta B}{B_{0}}$ : (a) West global absorption, (b) East global absorption, and (c) East first-pass 5-kG absorption. 
tion can be significant at the second-harmonic, but negligible at the fundarnental. The ray-tracing results are combined with the cavity-absorption model to predict the global ECRF absorption in the West end-cell. The calculations are in good agreement with the measurements.

\section{First-Pass A bsorption of the 5-kG ECRH}

The MECH code provides a simulation of the first-pass absorption of the 5-kG ECRH beam in the West end-cell. For the nominal plasma spatial structure, the code predicts that $72 \%$ of the $5-\mathrm{kG}$ power is absorbed on the first pass. About half of the absorbed power is deposited in the halo region, in correspondence with the limiter-scan experiment.

To model the ECRH propagation and absorption, we use the plasma structure model presented in figure 4.5, which is based on the measurements obtained in the previous chapter and discussed in section 3.1. The core contains average densities of $5 \times 10^{11} \mathrm{~cm}^{-3}$ cold-component electrons, $3.5 \times 10^{11} \mathrm{~cm}^{-3}$ warm-component electrons, $2 \times 10^{11} \mathrm{~cm}^{-3}$ super-hot-component electrons, and no hot-component electrons. We assume that the cold electrons have a $20-\mathrm{cm}$ parabolic profile and the other components are spatially uniform for $|x|<18 \mathrm{~cm}$. The halo is assumed to contain triangular density profiles, with peak values of $1.0 \times 10^{12} \mathrm{~cm}^{-3}$ hotcomponent electrons and $3.4 \div 10^{11} \mathrm{~cm}^{-3}$ super-hot-component electrons. The halo has been made somewhat larger than has been measured to reduce the density gradients and avoid problems with the ray-tracing calculations.

Figure 4.6(a) shows the ray trajectories projected into the $x-y$ plane. The numbers plotted on the rays indicate the power absorbed from the ray, with ' 1 ' corresponding to $10 \%$, ' 2 ' corresponding to $30 \%$, and so forth. The large density gradients in the halo region cause a significant amount of refraction. The spatial profile of the power deposition, flux mapped to the end-cell midplane, is shown in figure 4.6(b). The contours in this profile are normalized to the peak absorption, showing $90 \%$ of peak, $80 \%$ of peak, and so forth; the actual magnitudes of the contour levels, in $\mathrm{kW} / \mathrm{cm}^{2}$, are given in the upper-right corner of the figure. Deposition is strongest in the halo region closest to the 5 -kG heater, but the super-hot electrons continue to absorb power all along the beam trajectury. The halo region 


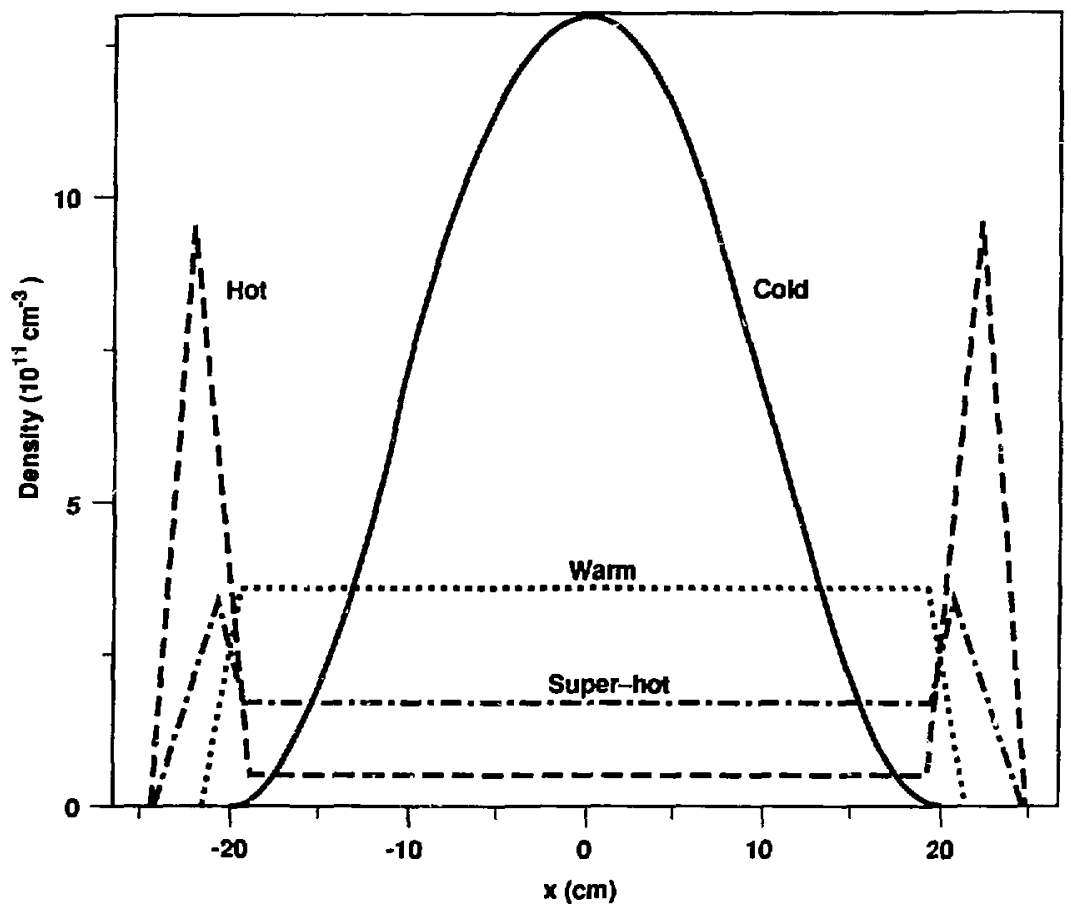

Figure 4.5. Midplane density profiles along the $\mathrm{x}$-axis for the MECH power-deposition calculation. 
remote from the 5-kG heater absorbs about $15 \%$ of the beam power; however, because of refraction, the deposition is distributed over a larger area and is not resolved on the contour plot. The first-pass absorption of the $5-k G$ beam is $\alpha_{1, x}$ $=72 \%$. A similar simulation using o-mode polarization gives an $\alpha_{1,0}=16 \%$. The power deposition contours fluinly show the strong absorption in the halo region.

When the midplane magnetic field is reduced to $\frac{\Delta B}{B_{0}}<-3 \%$, the super-hotcomponent plasma becomes annular with a larger halo super-hot-electron density. Using the results of section 3.3.3, we set the super-hot density to zero in the core region and to $5.5 \times 10^{11} \mathrm{~cm}^{-3}$ in the halo. We use the same hot-component density as in the nominal structure model, as similar amounts of warm-component diamagnetism are seen for most negative field offsets. The results of the modified simulation are shown in figures $4.7(\mathrm{a})$ and (b). Due to reduced absorption in the core, it is now possible to see the localized deposition in the portion of the halo remote from the $5-\mathrm{kG}$ heater. The single-pass absorption is smaller than for the nominal plasma structure, $\alpha_{1, x}=45 \%$. Further ray-tracing calculations show that about half of this power (20\%) is absorbed by the hot-component.

\section{First-Pass Absorption of the 10-kG ECRH}

The 10-kG ECRH launching structure, the uniform heater, produces a very broad radiation patteris. Only a very small fraction of the radiated power is absorbed on the first pass; the majority of the power simply misses the fundamental resonance within the plasma. The calculated first-pass absorption of the uniform heater output power is $21 \%$. In typical operation, this corresponds to only about $9 \%$ of the total incident ECRF power from both heating systems.

The results of the simulation are shown in figures $4.8(\mathrm{a}-\mathrm{b})$. Only the 100-eV core-region cold-electron population used in the previous section is assumed to be present at the $10-\mathrm{kG}$ field location. The spatial profile is flux mapped from the midplane to the axial location of the fundamental resonance. At this location, the plasma cross section is highly elliptical, with an $80-\mathrm{cm}$, horizontal major axis, and $8-\mathrm{cm}$, vertical minor axis. The ray trajectories in the $y-z$ plane are shown in figure $4.8(\mathrm{a})$. In this figure, only the rays launched from the center of the uniform heater are followed to improve the resolution of the simulation; in other 
a) 5-kG heater ray trajectorles

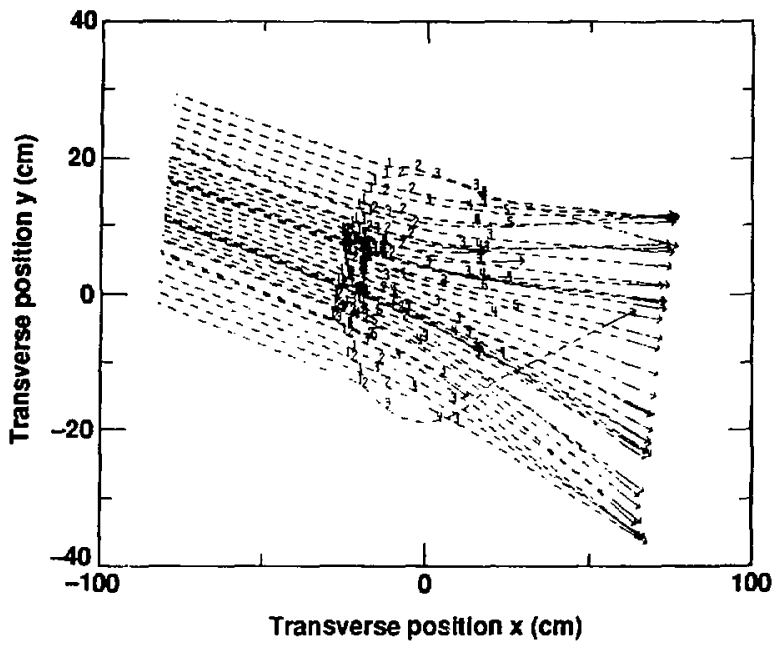

Absorption Levels

$110 \%$

$230 \%$

$3 \mathbf{5 0 \%}$

$470 \%$

$590 \%$

b) Power-deposition proflle

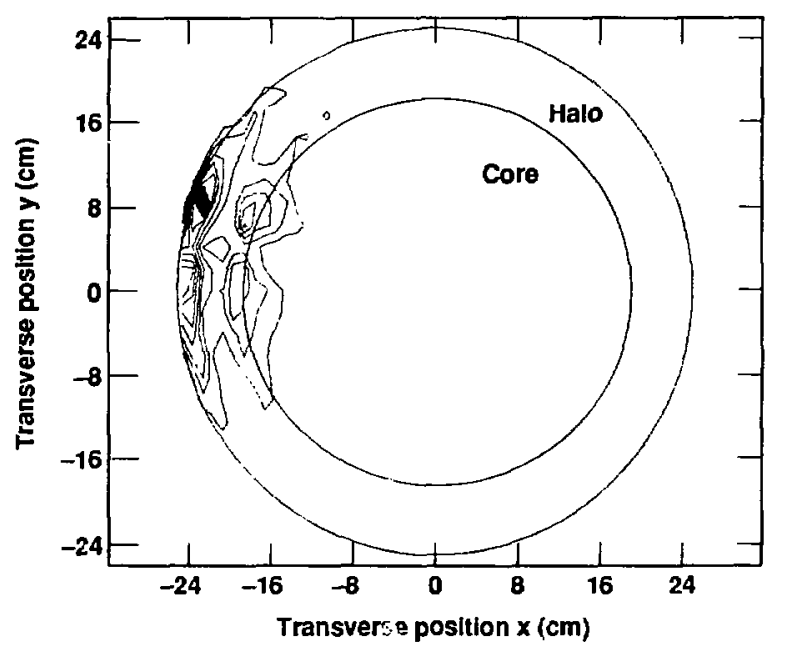

\begin{tabular}{|c|}
\hline Contour Levels $($ W/cm \\
\end{tabular}

Figure 4.6. Simulated first-pass absorption of the 5-kG ECRH beam: (a) ray trajectories, (b) power-deposition contours. 
a) 5-kG heater ray trajectories

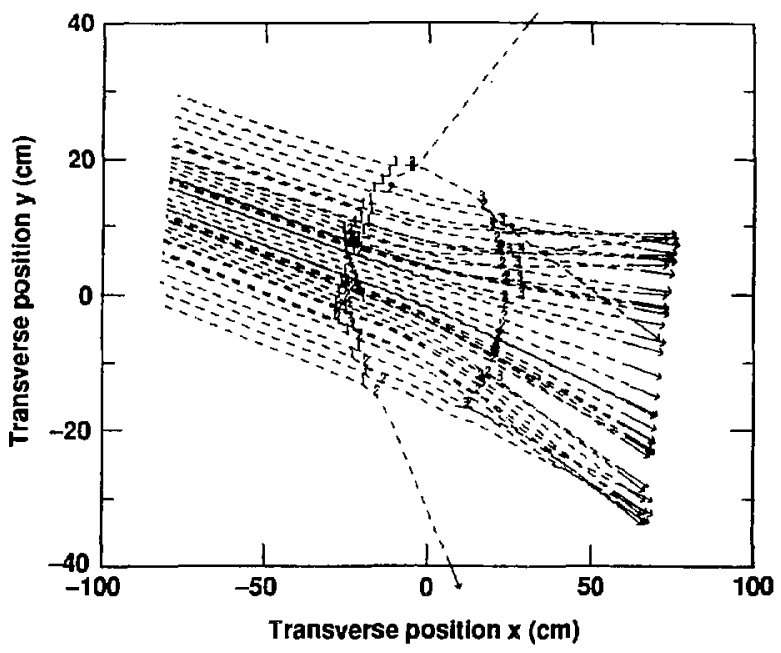

\begin{tabular}{|c|c|}
\hline \multicolumn{2}{|c|}{ Absorption Levels } \\
\hline 1 & $10 \%$ \\
\hline 2 & $30 \%$ \\
\hline 3 & $50 \%$ \\
\hline 4 & $70 \%$ \\
\hline 5 & $90 \%$ \\
\hline
\end{tabular}

b) Power-deposition profile

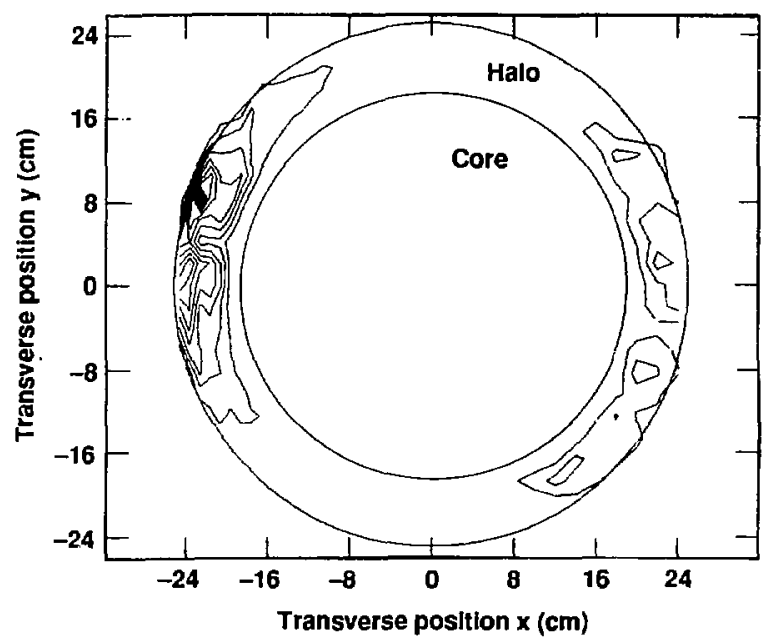

\begin{tabular}{|c|}
\hline Contour Levels $\left(W / \mathrm{cm}^{2}\right)$ \\
\hline 30 \\
61 \\
92 \\
122 \\
153 \\
184 \\
214 \\
245 \\
275 \\
\hline
\end{tabular}

Figure 4.7. First-pass 5-kG absorption with purely annular super-hot electrons: (a) ray trajectories, (b) power-deposition contours. 
simulations it was observed that ihe remaining off-center rays did not contribute to the absorption. Most of the $\mathbf{x}$-mode rays that intersect the fundamental resonance within the plasma are completely absorbed, while simulations with o-mode rays show only $16 \%$ absorption. Refractive effects cause some $\mathrm{x}$-mode rays incident on the lower portion of the plasma to be poorly absorbed as well. The uniform heater launches about $75 \%$ of its power in the $\mathrm{x}$-mode, and $26 \%$ of this power is absorbed at the fundamental resonance. The remaining $25 \%$ of the incident power in the o-mode is only weakly absorbed, and the overall first-pass absorption efficiency of the $10-\mathrm{kG}$ heater is $21 \%$. Figure $4.8(\mathrm{~b})$ shows the power deposition profile projected to the end-cell midplane. Because of refraction, power is more strongly deposited in the upper and lower edges of the plasma. The "band structure" of the absorption is introduced by the coarseness of the ray-tracing calculation; in reality, the bands would merge together into a continuous distribution. The basic structure of the absorption is nonetheless captured by the simulation. Despite the uniform radiation pattern of the 10 -kG heater, the power deposition profile is very asymmetric. This is extremely undesirable because it can lead to asymmetries in the plasma poteritial and radial transport of the center-cell ions ?

\section{Cavity Heating}

Cavity heating contributes significantly to the overall ECRF absorption, particularly when the diamagnetism is large. The measured perpendicular pressure at the plasma edge can correspond to a $\beta$ as large as $10 \%$. Thus, in the shallow magnetic well of the TMX-U end-cell, the second-harmonic resonance can have a large axial extent. We model the resonant surface as an elliptical cylinder with transverse dimensions as determined in the spatial structure study. The length of the cylinder is calculated from the measured stored energy per unit length $\left(w_{l}\right)$, obtained directly from the diamagnetism. The estimated resonant area is assumed to absorb the available power in equilibrium with the cavity loss area, which has been measured to be $A_{\text {lcss }} \approx 0.65 \mathrm{~m}^{2}$. This gives a conservative estimate for the cavity absorption, for relativistic and Doppler broadening also increase the absorptive area of plasma, particular for the super-hot-component electrons. At the fundamental resonance, however, the absorptive surface area is much less than 
a) 10-kG heater ray trajectories
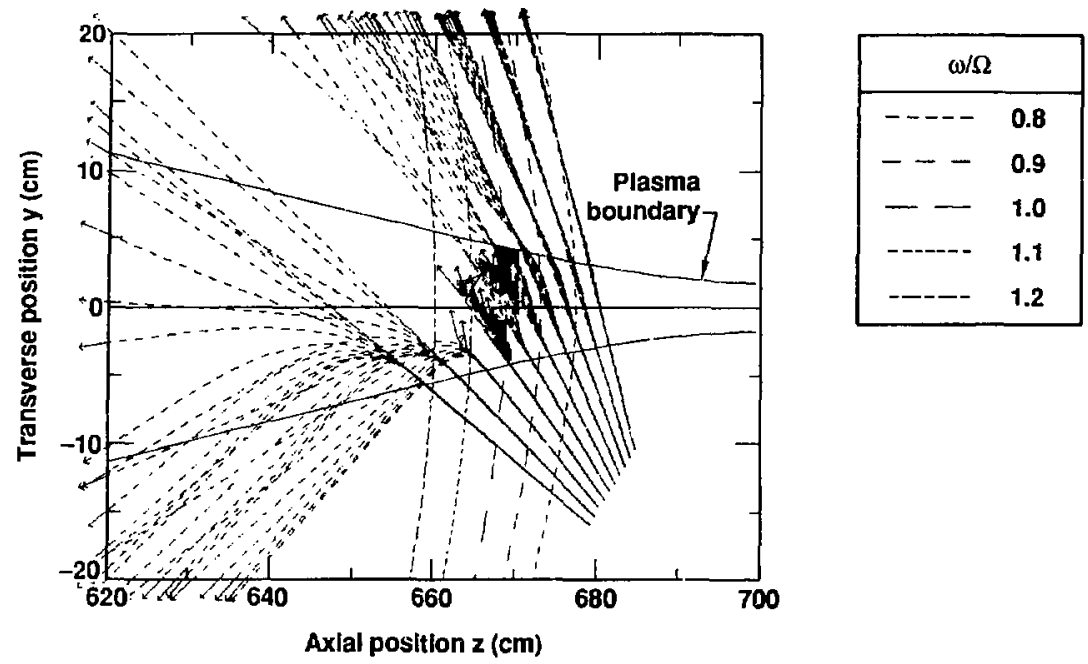

b) Power-deposition profile

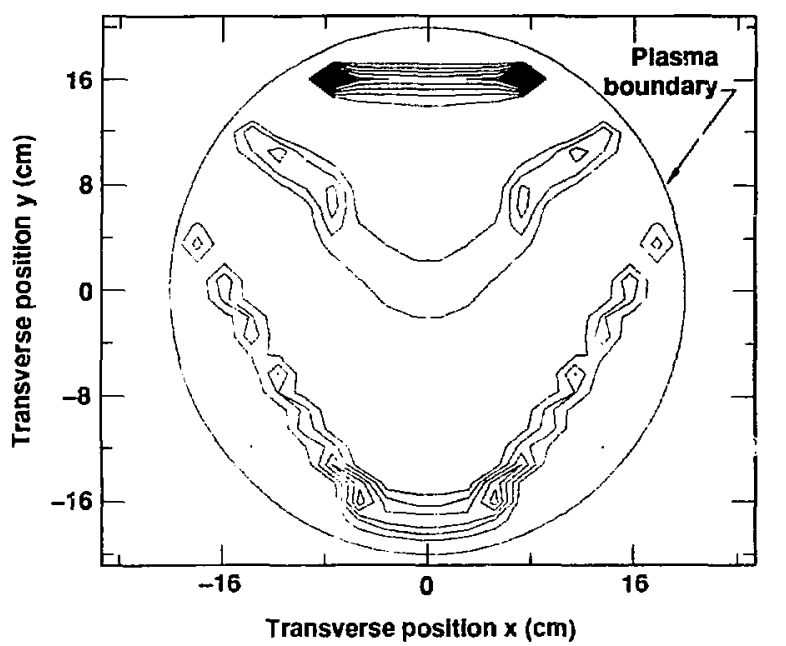

\begin{tabular}{|c|}
\hline Contour Levels $\left(W / \mathrm{cm}^{2}\right)$ \\
\hline$B$ \\
16 \\
25 \\
33 \\
40 \\
50 \\
57 \\
66 \\
74
\end{tabular}

Figure 4.8. Results of fundamental ray tracing: (a) ray trajectories, (b) power-deposition contours. 
the cavity loss area, and the cavity-heating contribution is small.

The plasma diamagnetism reduces the vacuum magnetic field $B_{v a c}$. For a long, thin plasma,

$$
B_{e q}=B_{v a c} \sqrt{1-\beta} \approx B_{v a c}(1-\beta / 2) .
$$

In the TMX-U end-cell plasma, the peak beta occurs at plasma edge and is given by

$$
\beta_{e}=h \frac{W_{l}}{A_{p} p_{0}},
$$

where $A_{\mathrm{p}}$ is the plasma cross-sectional area; $p_{0}=\frac{B_{0}^{2}}{2 \mu_{0}}$ is the energy density (or pressure) associated with the confining magnetic field at the end-cell midplane; $h$ is the spatial profile hollowness; and $W_{l}$ is the stored perpendicular energy per unit length measured directly by the diamagnetic loop. In chapter 3 , we determined $A_{p} \approx 2000 \mathrm{~cm}^{2}$, and $h \approx 2$. Now, we want to relate the plasma beta to the axial profile of the vacuum magnetic field $\left(B_{\text {vac }}\right)$ to determine the $z$ value where the magnetic-field depression produced by the peak plasma beta just equals the axial increase in the vacuum magnetic field; this will define the length of the resonant surface, $l_{\alpha}$. We approximate the vacuum magnetic-field variation near the midplane as parabolic:

$$
B_{v a c}(z)=B_{0}\left(1+\eta z^{2}\right)
$$

where $\eta \approx 0.0042 \% \mathrm{~cm}^{-2}$. Th! ss, we determine $l_{a}$ from

$$
\frac{B_{v a c}\left(l_{a} / 2\right)-B_{0}}{B_{0}} \equiv \frac{\beta_{e}}{2}
$$

Solving for $l_{a}$ gives

$$
l_{a}=2 \sqrt{\frac{h W_{l}}{2 \eta A_{p} p_{0}}} .
$$

Thus we can easily calculate the resonant surface area from the diamagnetism:

$$
A_{\text {res }}=\pi(a+b) l_{a},
$$

where $a$ and $b$ are the lengths of the elliptical plasma boundary at the midplane, as determined in chapter 3 .

The effective second-harmonic absorption area must take into account the finite single-pass absorption of the plasma, which is different for the two wave 
polarizations. The average single-pass absorption efficiency is given by a weighted sum over the polarizations:

$$
\bar{\alpha}_{1}=\frac{S_{\mathrm{cav}}^{o} \alpha_{1}^{o}+S_{c a v}^{x} \alpha_{1}^{x}}{S_{c a v}}
$$

where $S_{\text {cav }}=S_{c a v}^{o}+S_{c a v}^{x}$ is the cavity flux as defined in section 4.1.1. Typically, we measure $S_{\text {cav }}^{o} \approx S_{c a v}^{x} \approx S_{c a v} / 2$, so

$$
\bar{\alpha}_{1}=\frac{\alpha_{1}^{o}+\alpha_{1}^{x}}{2} .
$$

Thus, we can express $A_{a b s}$ in terms of the diamagnetism, $M$. For area in square meters and diamagnetism in units of $10^{5} \mathrm{Acm}$ :

$$
A_{\mathrm{a} b s}=\overline{\boldsymbol{\alpha}}_{1} A_{\text {res }}=0.88 \overline{\boldsymbol{\alpha}}_{1} M^{1 / 2} .
$$

However, the absorptive area at the fundamental resonance is small. The fundamental absorptive area is proportional to the resonant area,

$$
A_{\text {res }}=2 A_{\text {cold }} \frac{B_{0}}{B_{\text {res }}},
$$

where $A_{\text {cold }} \approx 0.1 \mathrm{~m}^{2}$ is the area of the cold plasma component at the end-cell midplane, and the factor of two accounts for the inboard and outboard fundamental resonances. The ratio of the magnetic fields appears to take into account the reduction in plasma area due to the higher field at the second-harmonic resonance, where $B_{\text {res }}=10 \mathrm{kG}$. Thus, $A_{\text {res }} \ll A_{\text {loss }}$, and the cavity absorption at the fundamental will be weak. Other factors also reduce the absorption. The electrons in this region of the end-cell have a low temperature, around $100 \mathrm{eV}$, so there is little relativistic or Doppler broadening of the resonance. Furthermore, the magnetic-field gradients are large, so any thermal line broadening that does occur has little effect on the absorptive area. We must also consider the limited accessibility of extraordinary-mode rays to the fundamental resonance. Only rays incident from the high-field side of the resonance can be absorbed; those incident from the low-field are reflected at the righi-hand cutoff ${ }^{25}$. Ordinary-mode rays, however, can reach the resonance from either side, but are only weakly absorbed. The absorptive area is

$$
A_{a b s, \omega_{c e}}=A_{r e s}\left(\frac{\alpha_{1, \omega_{c e}}^{x}+2 \alpha_{1, \omega_{c e}}^{o}}{2}\right),
$$


where the 2 in front of the o-mode absorption takes into account the two-sided access to the resonant surface. From the ray tracing we get the absorption coeffcients for $\mathrm{x}$ - and o-mode rays passing directly through the resonance, and $A_{a b \text {, } \omega_{c e}}$ $=0.058 \mathrm{~m}^{2}$.

The absorbed ca.jiy power is determined from the equilibrium between power flow out of the cavity through the effective loss aperture area, and power flow into the absorptive area of the plasma. We have shown that the second-harmonic absorptive area is dominant, so the power absorbed from cavity fields is

$$
P_{\text {cav }}=\frac{A_{a b b, 2 \omega_{c e}}}{A_{a b s, 2 \omega_{c e}}+A_{l o d}} P_{0, c a v},
$$

where $P_{0, \text { cav }}$ is the power available for cavity heating

$$
P_{0, \text { cav }}=\left(1-\alpha_{5}\right) P_{5}+\left(1-\alpha_{10}\right) P_{10}
$$

Here, $\alpha_{5}$ and $\alpha_{10}$ are the absorption afficiencies of the 5- and 10-kG heaters, which deliver incident powers of $P_{5}$ and $P_{10}$, respectively.

We can now calculate the global absorption efficiencies from the plasma diamagnetism, incident 5- and 10-kG ECRH powers, and the first-pass absorption efficiencies previously determined with the MECH code; the results are summarized in table 4.1. During nominal operation, the model predicts that $78 \%$ of the incident power is absorbed. The majority of the power is absorbed at the second-harmonic resonance on the first pass, but a significant fraction, $26 \%$, is absorbed from the cavity fields. For the low-neld case, the overall absorption is reduced to $62 \%$ by the decline in the first-pass second-harmonic absorption effciency; in this case cavity fields and first-pass absorption contribute equally to the second-harmonic absorption. At high-field offsets, the absorption is due entirely to the fundamental. Fundamental absorption in all cases is dominated by the weak first-pass absorption ( $10 \%$ of the total incident power), but increases somewhat because of cavity heating at the fundamental resonance. The calculated values are in good agreement with the measurements for all three cases. 


\begin{tabular}{|c||c|c|c|c|c|}
\hline Operation & $\omega_{c e}$ & $2 \omega_{c e}\left(1^{\text {st }}\right.$ pass) & $2 \omega_{c e}$ (cavity) & Total & Measured \\
\hline Nominal & 11 & 41 & 26 & 78 & $70-80$ \\
\hline Low Field & 12 & 26 & 24 & 62 & $55-65$ \\
\hline High Field & 15 & 0 & 0 & 15 & $10-20$ \\
\hline
\end{tabular}

Table 4.1. Summary of ECRH absorption efficiencies (all values in \%).

\subsection{Energy Storage}

In the TMX-U end-cell, energy is stored principally in the long-lived super-hotcomponent electrons, although a small fraction, 10-15\%, is stored in the hotcomponent electrons. The diamagnetism measures the stored energy per unit length, and we call the time derivative of the diamagnetism the confined power per unit length. To convert these measurements into total stored energy and confined power, which are typically expressed as percent fraction of the total incident ECRH power, we assume an effective hot-electron plasma length of 120 $\mathrm{cm}$, based on various measurements of the axial or pitch-angle distribution.

\subsubsection{Temporal Behavior}

Modulation of the 10-kG ECRH shows the relative influence of the two ECRH systems in driving the stored energy. Figures 4.9(a) and (b) show time histories of diamagnetism and confined power. Plainly, the 5-kG system is the dominant source of stored energy during the early portion of the pulse. The modulation shows the 10-kG contribution to be less than $20 \mathrm{~kW}$, while the $5-\mathrm{kG}$ system initially provides $60 \mathrm{~kW}$ of confined power. The $5-\mathrm{kG}$ confined power drops rapidly with time, while the relative contribution of the $10-\mathrm{kG}$ power increases. The change in the diamagnetism after the ECRH is turned off ideritifies the two components of the stored energy. The $12 \%$ rapid drop is associated with the hot-component electrons, while the remaining diamagnetism corresponds to the super-hot component. Energy storage is plainly dominated by the super-hot electrons.

During the brief hot-electron buildup, the hot-electron energy distribution is expected to be strongly distorted by the ECRH ${ }^{13}$, and the process occurs too quickly to make $\mathrm{x}$-ray measurements of the actual distribution. However, the 
PECE measurements of the super-hot-component line density and temperature show, at least qualitatively the mechanism of energy storage [see figures $4.9(\mathrm{c})$ and (d)]. Apparently, energy is stored initially by increasing the number of relatively low-energy $(\approx 100 \mathrm{keV}$ ) electrons. Thereafter, the line density is constant, and a fixed number of well-confined super-hot electrons store energy by continuously increasing their temperature.

Two mechanisms cause the confined power fraction to drop as the discharge progresses. During the first few milliseconds of the shot, the hot-electron plasma temperature is relati . ely low, less than $100 \mathrm{keV}$, and the dianagnetism is small. In this period, first-pass absorption of the 5-kG beam is efficient, and the stored energy rises rapidly. Once the plasma forms its equilibrium structure, the super-hotcomponent first-pass absorption drops as the imaginary part of the wavenumber, $k_{i}$, is decreased by the increasing temperature and diamagnetism of the superhot electrons ${ }^{10}$. Figure 4.10 shows $k_{i}$ as a function of $\frac{\Delta B}{B_{0}}$ (normalized to the second-harmonic) for varjous temperatures, calculated for a plasma density of $6 \times 10^{11} \mathrm{~cm}^{-3}$. As the temperature increases, the magnitude of the peak damping coefficient drops, and the location of the peak shifts to higher magnetic field due to the relativistic mass increase. Also, the plasma diamagnetism reduces the local magnetic field, further decreasing the line-integrated absorption. Absorption is decreased further by the strong distortion of the distribution associated with strong ECRH diffusion in velocity phase space ${ }^{36}$. Later in the discharge, as the first-pass absorption drops, cavity heating becomes dominant and the 10-kG ECRH has more influence on the stored energy. Another cause for the temporal decrease in the confined power fraction is competition between super-hotand hot-component ECRF absorption. Radial loss measurements indicate that hot-compnnent absorption increases with time, apparently due to increasing hotcomponent density in the halo (see section 4.4). Thus, as the discharge progresses, less power is stored in the super-hot component and more power is deposited in the poorly confined hot-component electrons in the halo.

The continuous increase in hot-electron temperature during the latter period of the discharge can be explained by the removal of the second-harmonic resonance from the core region by diamagnetism. The brief buildup in line density occurs during the period of plasma radial growth, as noted in section 3.2. Subsequently, 
(a) Diamagnetism

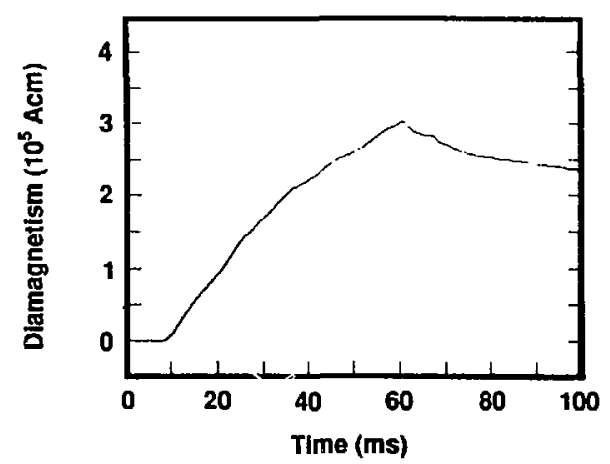

(b) Contined power

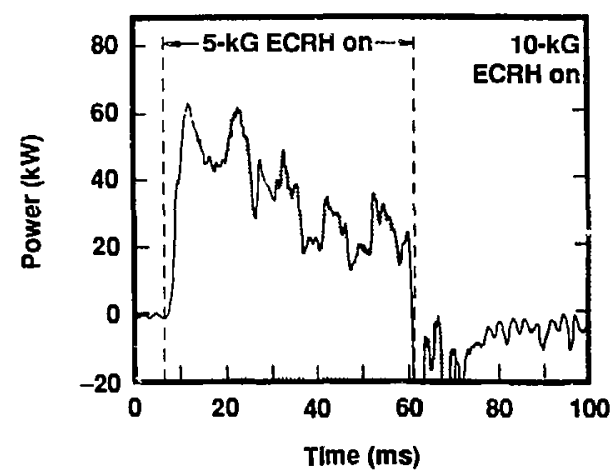

(c) Super-hot line density

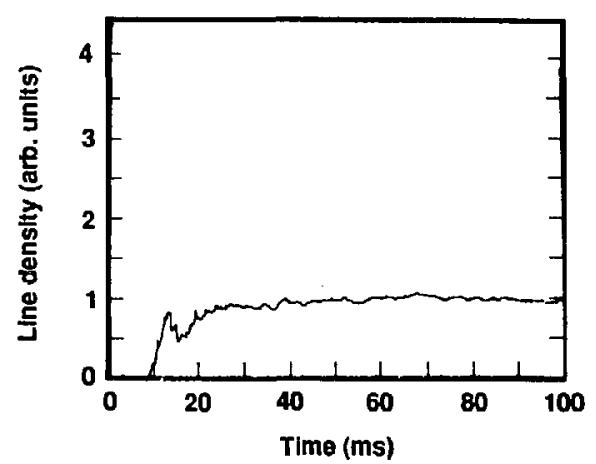

(d) Super-hot temperature

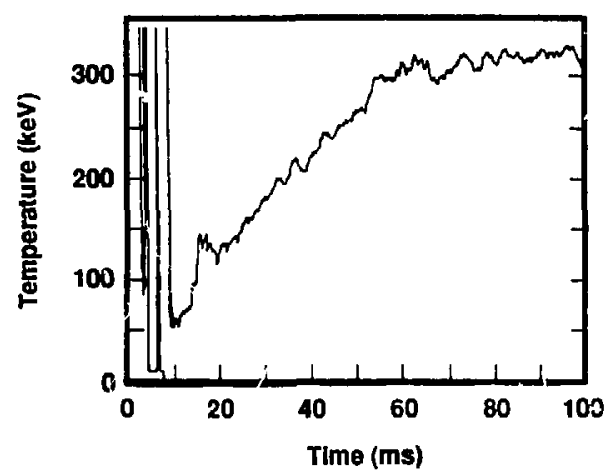

Figure 4.9. Time history of energy storage parameters: (a) diamagnetism, (b) confined power, (c) super-hot line density, (d) super-hot temperature. 


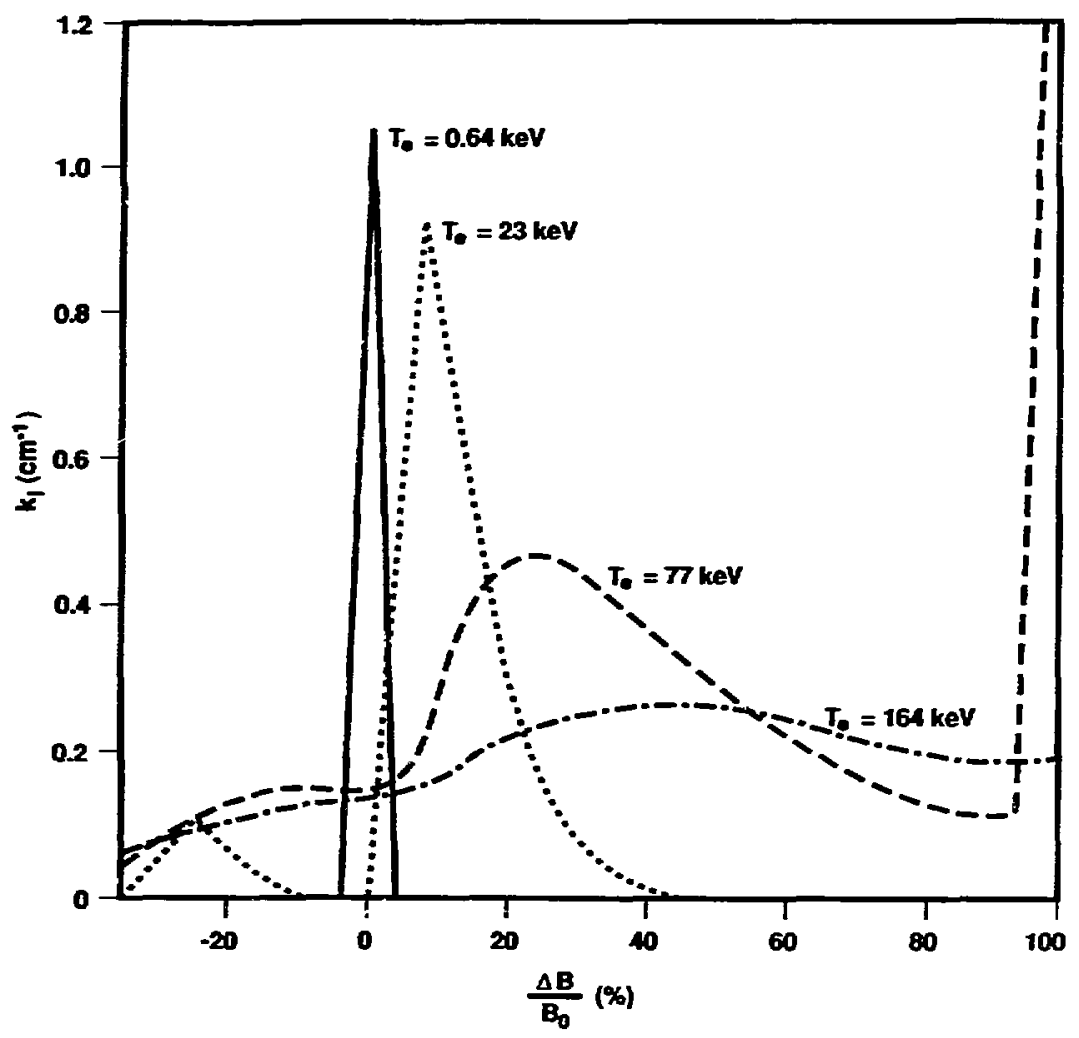

Figure 4.10. Imaginary part of the wavenumber versus $\frac{\Delta B}{B_{0}}$ for $28 \mathrm{GHz} \mathrm{ECRH}$. 
there is nis longer a second-harmonic resonance in the core. As a result, low-energy electrons are deprived of a power source and are rapidly lost axially, as evidenced by a brief early burst of energetic electron end-loss (discussed in section 4.3). However, electrons with sufficiently high energies can continue to absorb energy from the ECRH as a result of relativistic effects, specifically contributions to the damping coefficient from higher harmonics. Only a small fraction of the initially formed hot electrons survive this process, leaving behind only the low-density super-hot-component electrons.

\subsubsection{Spatial Structure}

The equilibrium spatial structure of the stored energy was discussed in detail in the section 3.1. Recall that the azimuthally averaged super-hot-component storedenergy profile is hollow, with about half of the stored energy contained in the halo region. The drop in stored energy associated with scrape-off of the halo region corresponds to an average $20-\mathrm{kW}$ reduction in confined power. Thus, the $30-\mathrm{kW}$ drop in global absorption with limiter radius described in section 4.1.3 is largely due to the reduced energy storage.

\subsubsection{Variation with Vacuum Magnetic Field}

The dependence of confined power fraction on magnetic-field setting is shown for the West end-cell in figures 4.11. The 5-kG confined power fraction has a peak value of about $30 \%$ for field offsets around $-1.5 \%$. It is not sensitive to the magnetic field. and the 5-kG system continues to contribute to the stored energy at the low and high magnetic-field offsets. The peak confined power fraction is around $35 \%$. When the 5- and 10-kG systems are operated together, the results are identical to that of the $5-\mathrm{kG}$ system to the resolution of the data set. However, the $10-\mathrm{kG}$ system confined power fraction is sharply peaked around $-1.5 \%$; the peak value is about $20 \%$, and at low and high field offset the confined power fraction goes to zero. A strong synergy is apparent: the $5-\mathrm{kG}$ heater facilitates the confinement of the $10-k G$ energy. This synergy is particularly striking when $\frac{\Delta B}{B_{0}}<-2 \%$.

Due to the radial loss mechanism, hot-electron confinement is better in the 


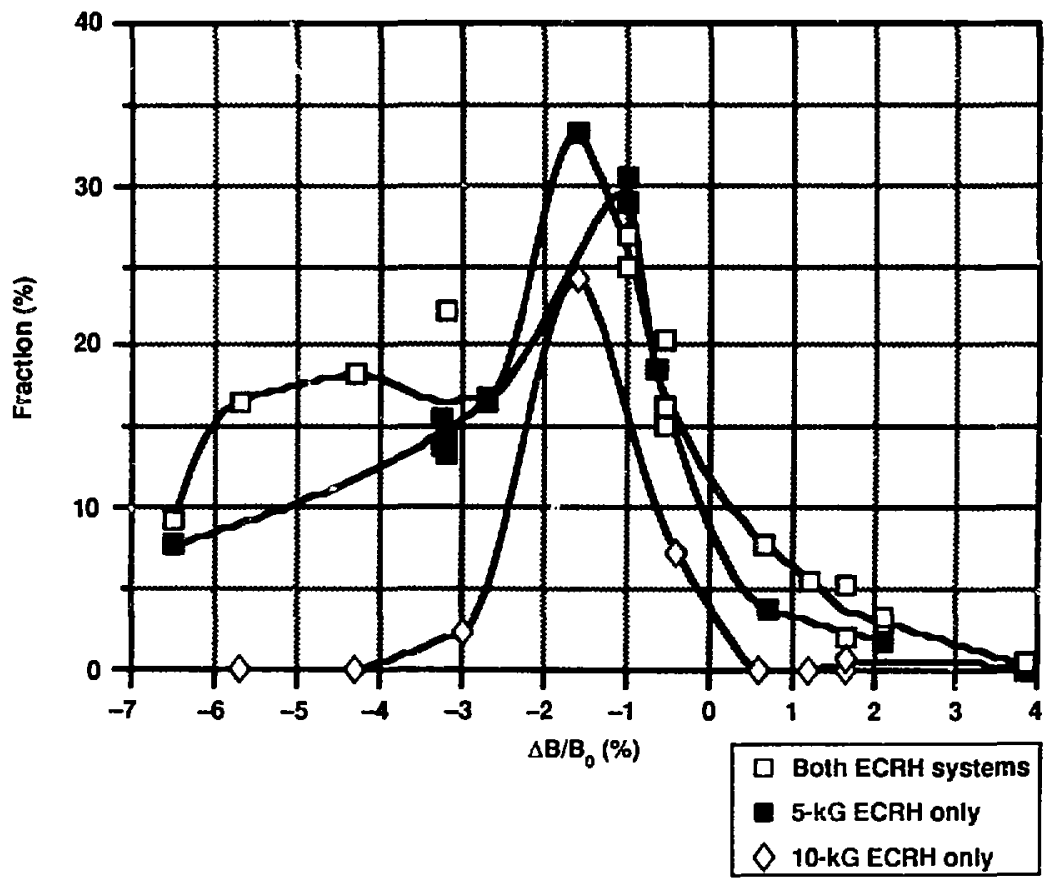

Figure 4.11. Confined power fraction versus $\frac{\Delta B}{B_{0}}$ in the West end-cell. 
core region than in the halo. Depressing the magnetic field moves the secondharmonic resonance into the halo region for the entire discharge, and the superhot-component plasma becomes annular. Then, more energy is absorbed by the poorly confined hot-electron component and less energy is stored in the super-hot electrons. This hypothesis is supported by the behavior of the radial losses, which increase as $\frac{\Delta B}{B_{0}}$ is decreased (see section 4.4).

The low confined power fraction for independent operation of the 10-kG system is not well understood. A possible explanation is supported by the following arguments. At large negative field offsets, 10 -kG power accountability is poor, as described in section 4.5. The 10-kG power appears to strongly drive the halo density, as indicated by comparison of figures $3.18(a)$ and (b). Therefore, we suspect that the 10-kG ECRH creates and is absorbed by a poorly confined halo plasma component that has not been characterized. Such a component is apparently not present when the 5-kG ECRH is operated, so the 10-kG confined power fraction incrrazes.

\subsection{Axial Losses}

\subsubsection{Axial Loss Diagnostics}

Bolometer Array An array of three bolometers is mounted in each TMX$\mathrm{U}$ end-fan. In the West fan, the bolometer positions map to end-cell roordinates $-4.2,-11$, and $-18 \mathrm{~cm}$ along the $\mathrm{y}$ axis. Each bolometer consists of two platinum wire resistor grids. One grid, the sensor, is exposed to the end-loss flux while the other acts as a reference, and the two grids are connected in a bridge circuit. The change in resistance of the sensor grid is proportional to the temperature rise of the wire, which in turn is proportional to the axial loss fluence. The bolometer signal is acquired into the TMX-U data base and converted to flux by differentiation ${ }^{26}$. The power-loss flux is converted to power by forming a weighted sum of the three bolometer signals.

Electron End-Loss Spectrometer (EELS) A cryogenically cooled surfacebarrier diode is used to do electron spectroscopy. The diode produces pulses 
with amplitudes proportional to the electron energy. The amplified diode signal is input to a fast pulse-height analysis system to provide the electron end-loss energy spectrum. The system uses upper- and lower-level discriminators which were typically set to $150 \mathrm{keV}$ and $11 \mathrm{keV}$, respectively. The EELS is mounted at the center ( $\mathrm{z}$ axis) of the East fan of TMX-U ${ }^{27}$.

Electrostatic End-Loss A nalyzer (ELA) The ELA is a gridded, electrostatic analyzer that provides the low-energy spectrum of either electron or ion endloss. It consists of in entrance aperture followed by repeller grids that remove either electrons or ions, as desired, a swept analyzer grid to modulate the desired species, and a final secondary-electron suppressor grid. The energy range of the spectrometer is determined by the analyzer grid sweep voltage, which can be as large as $3 \mathrm{kV}$, while the sweep rate limits the temporal resolution to about $4 \mathrm{~ms}$. The ELA is mounted near the center ( $\mathrm{z}$ axis) of the East end-fan of TMX-U ${ }^{28}$.

Ion End-Loss Diagnostics Two other diagnostics provide routine measurements of ion end-loss parameters. Arrays of Faraday cups provide spatial profiles the ion end-loss flux. Each cup consists of a 1.3-mm diameter metal cup covered with a suppressor grid ${ }^{29}$. The grid is biased at $-3 \mathrm{kV}$ to repel low-energy electrons. Ion energy spectra are provided by the End-Loss Ion Spectrometer (ELIS). The ELIS resolves ions of different energies with parallel electric and magnetic fields. The plasma potential and ion temperature can be determined from the ion energy spectrum ${ }^{30}$.

\subsubsection{Axial End-Loss Characteristics}

During the equilibrium portion of a discharge, the axial end-loss principally consists of cold ions, warm electrons, and cold electrons. Hot electrons are also present in the axial loss, but they carry a negligible amount of power. We have determined the relative contributions of the three species to the power-loss flux by combining EELS and ELA spectra for the electrons, and ELIS spectra for the ions. Most of the loss power flux, 90\%, is split between the cold ions and warm electrons. The ion temperature is quite low, around $30 \mathrm{eV}$, but the ions carry energy out of the plasma by falling down the positive plasma potential, which is typically in 
the range of $200-400 \mathrm{eV}$. The warm-electron current density is nearly an order of magnitude smaller than the ion flux, but carries equal power flux because of its higher temperature, typically $3 \mathrm{keV}$. The cold electrons contribute only about $10 \%$ of the loss power flux. Although they are the dominant source of electron current density, their temperature is low, typically $100-200 \mathrm{eV}$. These end-loss characteristics are typical of TMX-U operation with ECRH only; the character of the losses is quite different during standard thermal-barrier operation in which the sloshing-ion beams are operated and the plasma potential is much higher ${ }^{31}$.

\subsubsection{Temporal Variation}

Typical time behavior of axial power loss is shown with the 5- and 10-kG heating waveforms in figures $4.12(\mathrm{a}-\mathrm{c})$. During the first few milliseconds of hot-electron build up, only the 5-kG system is active and the axjal loss fraction is quite large, about $33 \%$. Thereafter, the loss fraction drops substantially. For 5-kG ECRH the axial-loss fraction is $15 \%$, while the $10-\mathrm{kG}$ system drives the axial losses somewhat more efficiently, with a power-loss fraction of $20 \%$.

The energy spectrum of the high-energy electron end-loss changes rapidly during the first few milliseconds of the hot-electron buildup. The spectrum obtained from the EELS durirg the first $10 \mathrm{~ms}(10-20 \mathrm{~ms})$ of the buildup is shown in figure 4.13(a). Typically, the initial spectrum corresponds to a single temperature in the range of of 8-12 keV, then rapidly separates into a two component distribution dominated by the warm-component electrons at $2-5 \mathrm{keV}$ and a much smaller quantity of hot-component electrons, as shown in figures 4.13(b) and (c). The time variation of the low-energy electron spectra, as measured with the ELA, has not been aralyzed because the available data set is very small. A typical equilibrium ELA spectrum is shown in figure 3.20(a).

These measurements further support the scenario for rapid plasma growth presented in chapter 3. During the initial hot-electron formation, hot electrons are present in the core region. Briefly, the confinement of these electrons is dominated by axial loss. As plasma diamagnetism rapidly pushes the vacuum secondharmonic resonance out of the core region, the hot electrons disappear, leaving behind the equilibrium warm-, cold-, and super-hot electron populations. 


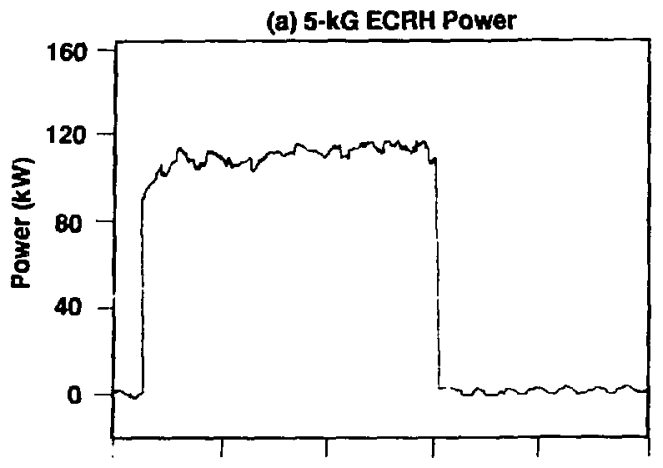

(b) 10-kG ECRH Power

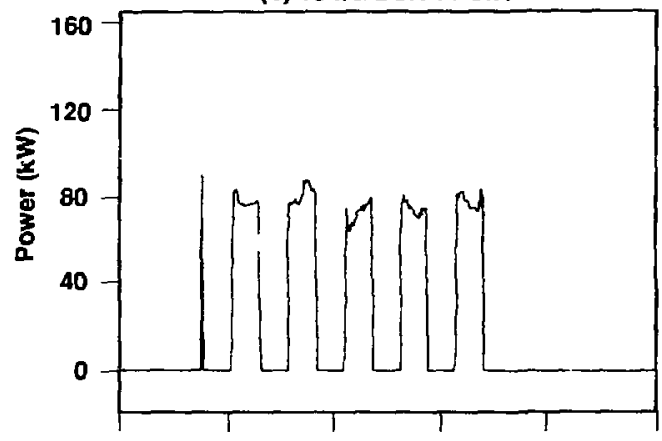

(c) Axlal Power Loss

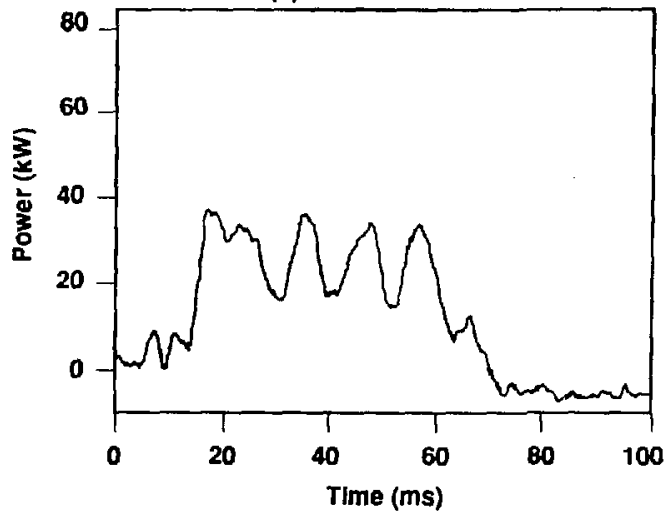

Figure 4.12. Time history of the axial power loss: (a-b) ECRF heating waveforms, (c) axial power loss. 


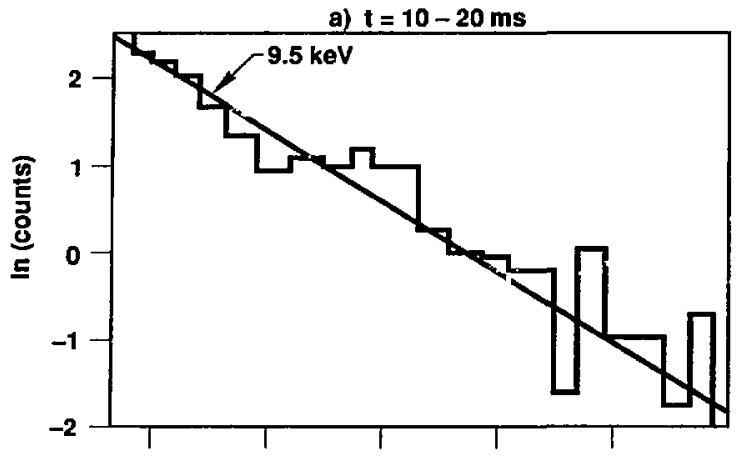

b) $\mathbf{t}=\mathbf{2 0}-\mathbf{3 0} \mathrm{ms}$

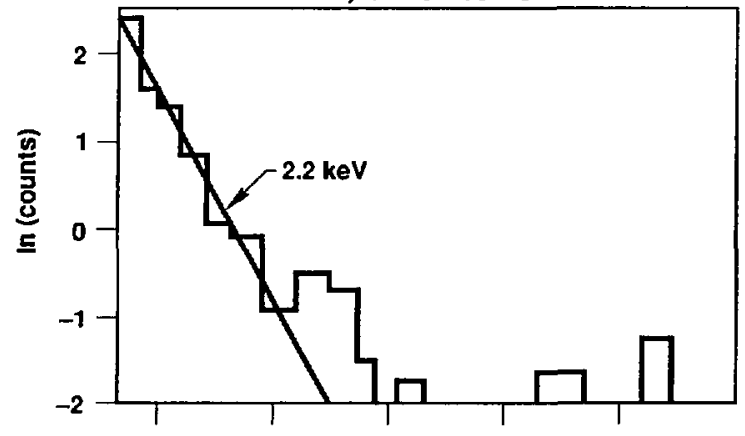

c) $t=30-40 \mathrm{~ms}$

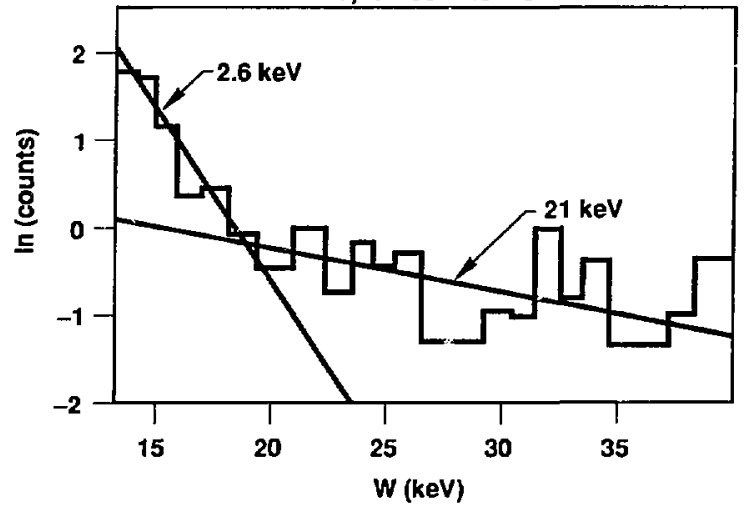

Figure 4.13. EELS electron energy spectra: (a) 10-20 ms, (b) 20-30 ms, (c) 30-40 ms. 


\subsubsection{Spatial Structure}

Spatial information is provided by the fixed array of bolometers on the West endfan [see figures $4.14(\mathrm{a}-\mathrm{c})$ ]. During the equilibrium period, the array shows a profile that can be fit by a 16-cm Gaussian, consistent with the expected size of the core region. Thus, the losses come principally from the core region, while little power is lost axially from the hot-electron halo. During the plasma build up, the loss profile is much narrower.

\subsubsection{Variation with Vacuum Magnetic Field}

The energy distribution of the electron axial losses in the East end-fan of TMX-U shows interesting changes as the magnetic field is varied. These results have been discussed previously ${ }^{27}$, but are summarized here for completeness (see figure 4.15). The loss of electrons with energies in the range of 55-102 keV, which we identify with the hot-component electrons, has a very strong dependence on the magneticfield setting. For positive field offset, there is little hot-electron buildup and the losses are small. When the second-harmonic resonance is introduced, the losses increase greatly, with a maximum occurring at a field offset of $-1.5 \%$. As the field is further decreased, the losses also decrease, particularly as the field is reduced below $-3 \%$. The modulation shows the dominance of the $5-\mathrm{kG}$ system in driving the hot-component losses; the peaking near $\frac{\Delta B}{B_{0}}=-1 \%$ is produced by strong firstpass heating. However, the loss of lower energy electrons in the range of $13-30 \mathrm{keV}$, which we identify with the warm-component plasma, shows no clear dependence on $\frac{\Delta B}{B_{0}}$. At high field offsets, where there is no hot-electron buildup, the data suggest that the 10-kG ECRH is a more efficient driver of the warm electrons. It is possible that the warm electrons are created by fundamental heating.

These results confirm our models of plasma buildup and equilibrium. As discussed, for nominal field conditions a burst of energetic electrons is lost axially during the initial plasma formation at the magnetic axis. Thereafter, the loss rate rapidly decreases to a steady value. When the initial second-harmonic resonance is moved out to a large radius, this first spike is not present and the loss count of the energetic electrons drops.

The variation of the total axial power loss is also of interest. The West fan 
(a) $y=-4.2 \mathrm{~cm}$

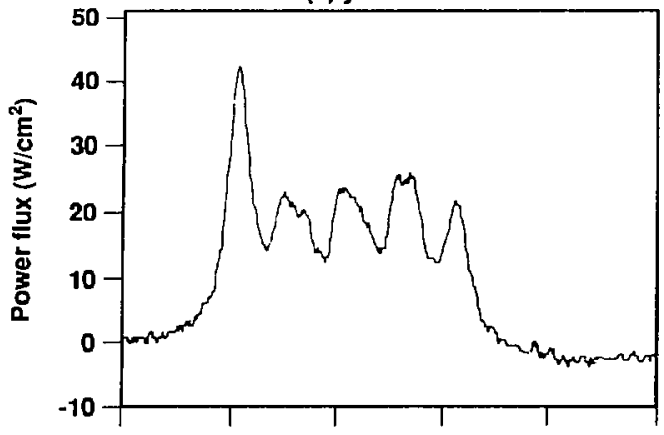

(b) $y=-1$ i cm

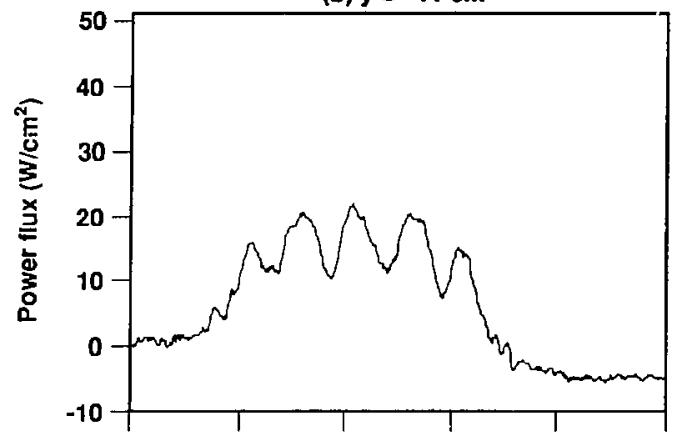

(c) $y=-18 \mathrm{~cm}$

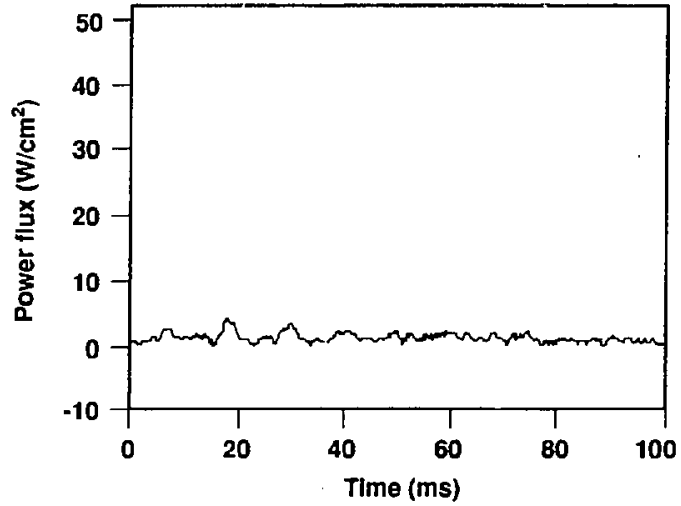

Figure 4.14. Time histories of bolometer array signals: (a) $y=-4.2 \mathrm{~cm}$, (b) $y=-11$ $\mathrm{cm}$, (c) $y=--18 \mathrm{~cm}$. 


\section{a) 10-kG modulated operation}
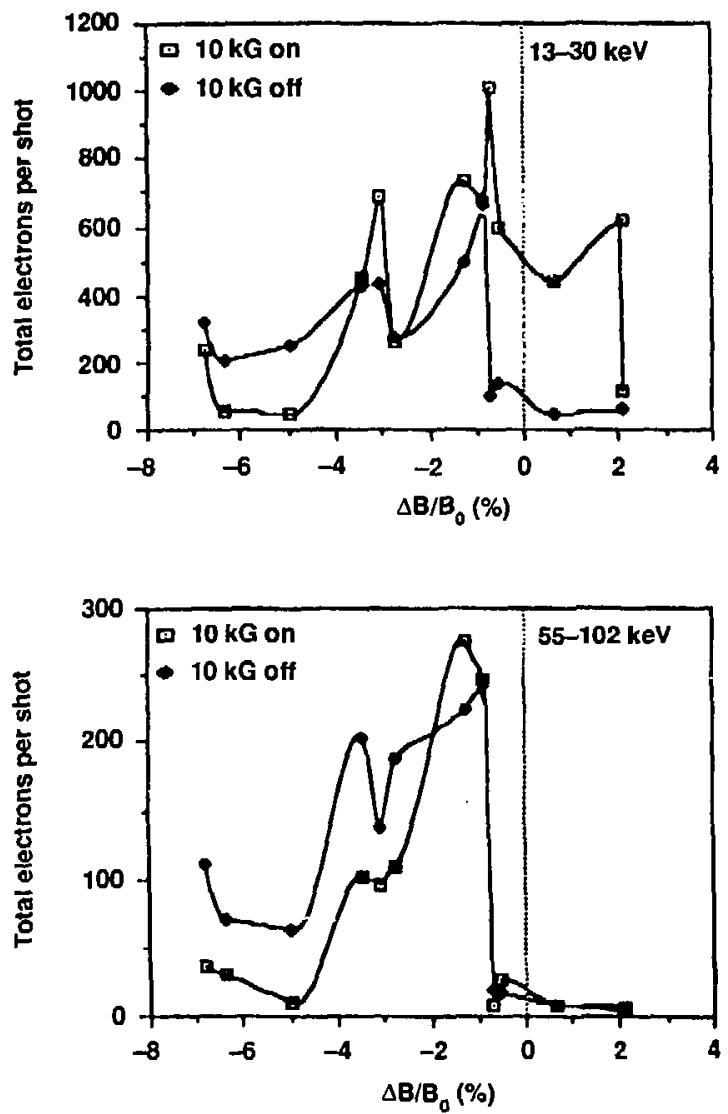

b) 5-kG modulated operation
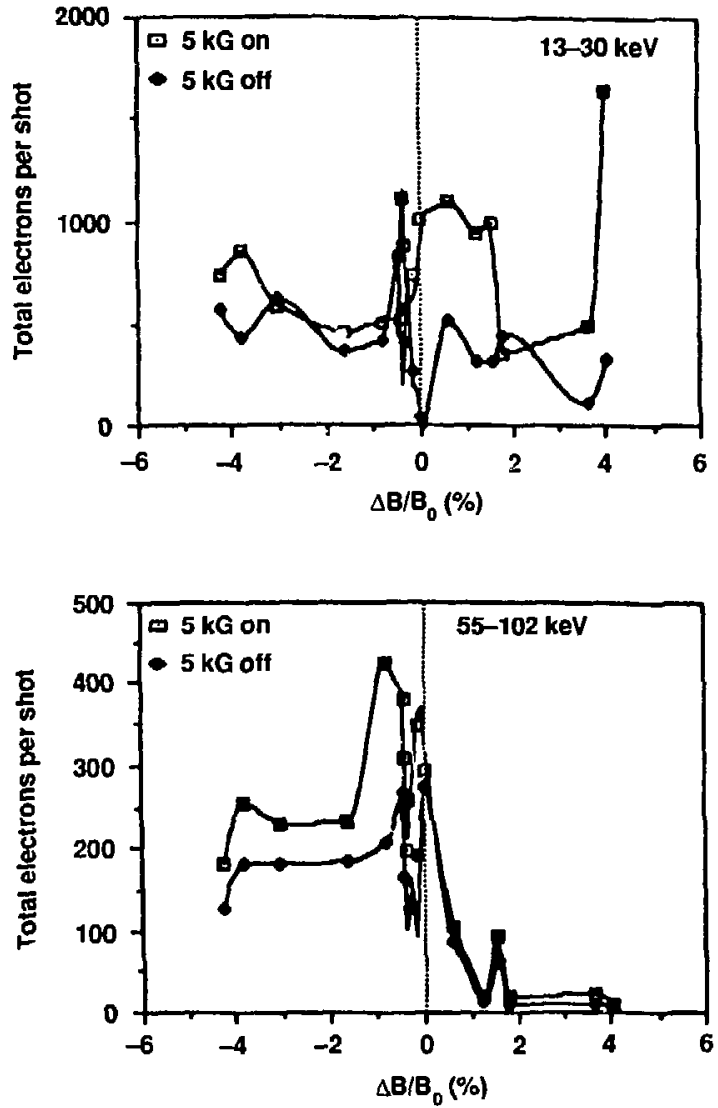

Figure 4.15. Variation of axial electron losses with field offset: (a) 10-kG ECRH modulated operation, (b) 5-kG ECRH modulated operation. 
results are shown in figure 4.16. The data show a loss fraction of about $8-13 \%$ at high field, rising to a maximum of $26 \%$ near $\frac{\Delta B}{B_{0}} \approx-1.5 \%$, then dropping to $17-23 \%$ for low field offsets. There is some difference between the efficiencies of the two ECRH systems; the $10-\mathrm{kG}$ system is a slightly more efficient driver of axial power loss.

The axial power loss, dominated by the warm-component electrons, shows little resonant structure. As long as a magnetic field is present within the plasma volume, the warm component remains nearly constant, both in temperature and density, and the losses remain the same. This indicates that the warm component is powered largely by fundamental ECRH or cavity-heating at the second hiarmonic.

\section{Axial Confinement}

The energy confinement time for the warm-component electrons in the core region can be estimated from the bolometer measurements of the axial power-loss flux. The loss flux $S_{a x}$ is related to the confinement time $\tau_{E}$ by a line integral along a magnetic flux tube:

$$
\left.S_{a x}\right|_{B_{1}}=B_{1} \int \frac{n_{w} T_{w}}{\tau_{E}} \frac{d s}{B},
$$

where $n_{w}$ and $T_{w}$ are the warm-electron density and temperature, respectively; $B_{1}$ is the magnetic field at the point of measurement (the end-fan); and $B$ is the magnetic field along the flux tube. We simplify the equation by taking the axja] profile as uniform with a length, $l_{h}=120 \mathrm{~cm}$, to obtain

$$
\tau_{E} \approx \frac{n_{w} T_{w} l_{h}}{\left.S_{a x}\right|_{B_{0}}} .
$$

Here, $\left.S_{a x}\right|_{B_{0}}=\left.\frac{B_{0}}{B_{1}} S_{a x}\right|_{B_{1}}$ is the axial power-lcas flux mapped to the end-cell midplane, where the magnetic field is $B_{0}, T_{w}$ is obtained from the EELS. $n_{w}$ is estimated from the interferometers as described in section 3.1. As discussed, the warm-component electrons produce about $45 \%$ of the axial power loss flux. Following this procedure for tive typical discharges yields $\tau_{E}=0.65 \pm 0.1 \mathrm{~ms}$.

If confinement is dominated by classical collisional diffusion into the mirror loss-cone, the warm electron energy confinement time should be roughly comparable to the $60^{\circ}$ pitch-angle scatter time. The warm electrons will scatter on other 


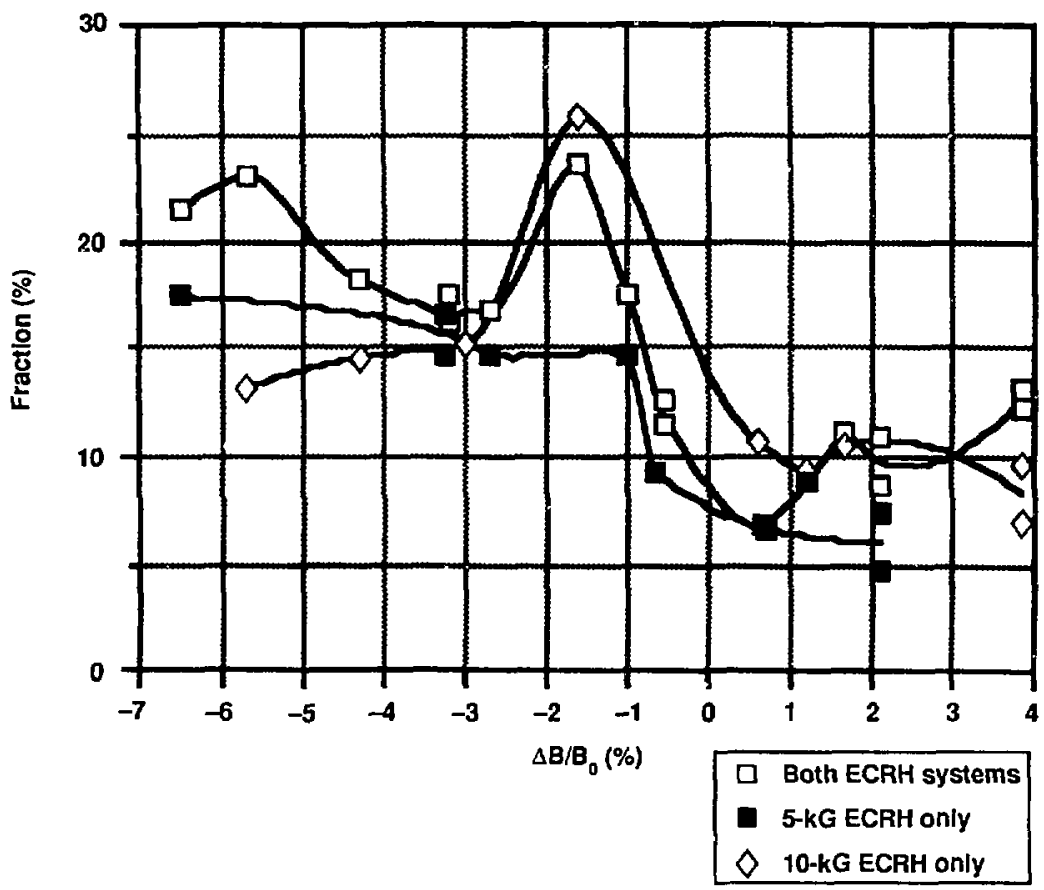

Figure 4.16. Axial loss fraction versus $\frac{\Delta B}{B_{0}}$ in the West end-cell. 
warm electrons, cold electrons, anci cold ions:

$$
\frac{d n_{w}}{d t}=n_{w}\left\langle n_{w a r m}\langle\sigma v\rangle_{w w}+n_{c o l d}\langle\sigma v\rangle_{w c}+n_{i o n}\langle\sigma v\rangle_{w i}\right) \equiv \frac{n_{w}}{\tau_{E}},
$$

where $\langle\sigma v\rangle_{w w},\langle\sigma v\rangle_{w c}$, and $\langle\sigma v\rangle_{w i}$, are the scattering cross-sections for warm electrons on warm electrons, cold electrons, and ions, respectively. Using some convenient estimates of the $90^{\circ}$ scattering cross-sections ${ }^{32}$, dividing by $\sin ^{2} 60^{\circ}$ to account for the $4: 1$ mirror loss cone ${ }^{33}$, and noting that $n_{\text {ion }}=n_{w}+n_{\text {cold }}$,

$$
\tau_{E} \approx \frac{T_{w}^{3 / 2}}{n_{\text {cold }}+0.66 n_{w}} .
$$

Obtaining estimates of the cold-electron density from the interferometers as in section 3.1, we obtain estimates of the expected $\tau_{E}$ for the same five discharges used above. The average value is $0.50 \pm 0.05 \mathrm{~ms}$.

Thus, we conclude that classical pitch angle diffusion into the mirror loss cone is the dominant loss process for the warm electrons in the core region. Furthermore, if we assume that the hot-component electrons in the core region also have classical axial confinement, we can use the EELS data to estimate their density. Exponential fits to the high energy spectra give a time-averaged relative loss flux for the hot- and warm-electron components, $j_{h o t} / j_{w} \approx 2 \times 10^{-3}$. We relate this to the temperature and densities of the components,

$$
\frac{j_{h o t}}{j_{w}}=\frac{n_{h o t}}{n_{w}}\left(\frac{T_{w}}{T_{h o t}}\right)^{2 / 2}
$$

Thus, taking the temperature ratio $T_{\text {hot }} / T_{w} \approx 10$, we get the density ratio,

$$
\frac{n_{\text {hot }}}{n_{w}} \approx 2 \times 10^{-3} 10^{3 / 2}=0.063 .
$$

So the core density of hot electrons is about $6 \%$ of the warm density, or roughly $2 \times 10^{10} \mathrm{~cm}^{-3}$. The loss spectra thus indicate a negligible density of hot electrons in the core region.

\subsection{Radial Losses}

Radial loss measurements were made with the HEED probe, which is described in detail in chapter 2 . In this section, we examine the neasurements of radial 
loss power, temperature, and density as a function of time, midplane radius, and magnetic-field offset. Next, the theoretical aspects of radial confinement are analyzed in detail with Fokker-Planck simulations and calculations of the radial transport rate. The theoretical results are quite close to the measured energyconfinement time.

\subsubsection{Temporal Variation}

Figures 4.17(a-c) show the time histories of power, temperature, and density for the radial losses. Radial loss begins promptly with the hot-electron buildup. During the first few milliseconds of the buildup, while the plasma is growing radially, the loss temperature is higher, typically $40-50 \mathrm{keV}$. During the remainder of the discharge there is usually little change in the temperature until the ECRH is turned off, whereupon the loss temperature rises sharply. Typically, the temperat ure will drop to a fairly constant value during the equilibrium portion of the discharge, while the density rises, although it can saturate or even drop during the last 10-20 ms of the ECRH. Radial losses continue after the ECRH is turned off, and during the subsequent $10-20 \mathrm{~ms}$, about $200 \mathrm{~J}$ of energy is lost-a significant fraction $(60-70 \%)$ of the hot-component stored energy. Presumably, the remainder is lost in the other loss plume (see section 4.4.2).

The prompt appearance of radial loss suggests the presence of an electron radial transport mechanism that carries power from the plasma in the core region onto the open drift surfaces. The higher energy of the radial losses during the plasma growth period is analogous to the behavior of the axial losses, again suggesting radial transport. The losses continue to rise slowly during the discharge as the halo density increases and traps more of the ECRF power. The stronger hot-component absorption competes with the super-hot-component absorption, reducing the confined power fraction. After the ECRH is turned off, the losses continue until the hot-component electrons have all been lost from the balo. The radial power loss during this period compares well with the derivatuve of the diamagnetism. 

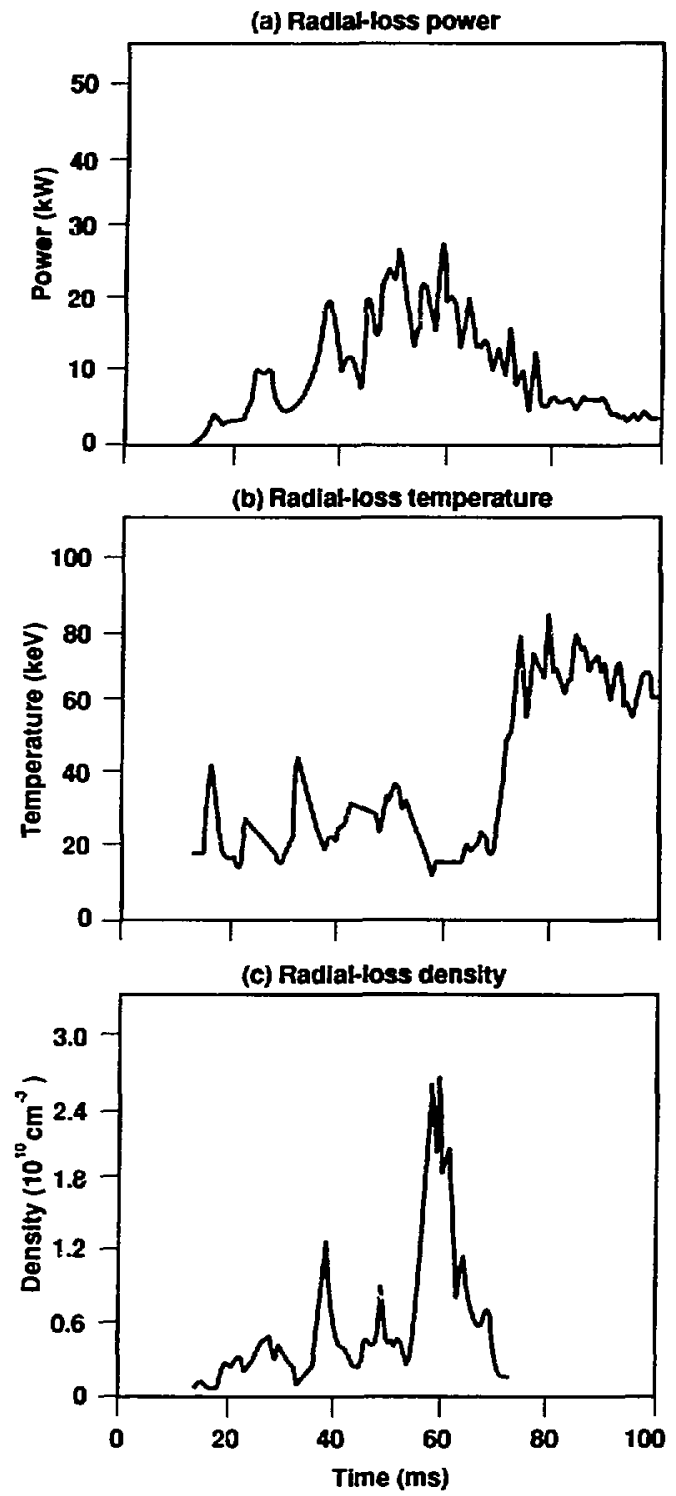

Figure 4.17. Temporal variation of three radial loss parameters: (a) power, (b) temperature, (c) density. 


\subsubsection{Spatial Structure}

Radial energy loss data was obtained for the West end-cell with the HEED probe during the limiter probe scans. The data is shown in figures $4.18(\mathrm{a}-\mathrm{d})$ as function of probe midplane radius. Altinough the radial losses quickly fall to zero for the scans in the $\phi=50^{\circ}, 60^{\circ}$, and $70^{\circ}$ orientations, they continue to a much larger radius for the $90^{\circ}$ orientation. Data from other TMX-U operation indicate that these losses are always present; when the HEED probe is not inserted, they are visible as thick target $\mathrm{x}$-ray emissions on the diamagnetic loop structure ${ }^{18}$.

Contours of the radial energy loss are shown in figure 4.19, together with some selected $65^{\circ}$ drift surfaces. The contours clearly show the presence of a loss plume that carries a significant amount of power. The measured spatial structure shows the influence of the open drift surfaces.

Open drift surfaces exist on both sides of the plasma, and damage marks and melting on the diamagnetic loop support structure indicate that two radial loss plumes are present. The orientation of these marks is in agreement with calculations of the open drift-surface trajectories. Because of the strong first-pass absorption of the 5-kG beam, however, the plume formed on the side closest to the 5-kG heater should contain the majority of the power. The HEED probe is positioned to monitor this plume. Ray-tracing calculations show only small power deposition in the halo plasma remote from the 5-kG ECRH beam, but cavity heating should power both plumes equally. Both heating mechanisms contribute about equally to the total absorption, so we can estimate the total radial loss power by multiplying the HEED power measurements by 1.4 .

\subsubsection{Variation with Vacuum Magnetic Field}

During the B-field scan experiment, the HEED probe obtained data on the radial loss temperature and power in the West end-cell. The electron radial loss temperat ure results are shown in figure 4.20(a). The values plotted are averages taken in the latter half of the shot in the interval 30-60 ms. The temperature decreases as the magnetic field is decreased, with the majority of the drop occurring in the vicinity of about $-3 \%$. The total electron radial power loss fraction is shown in figure 4.20 (b). At high fields where the overall ECRF absorption is small, the 
(a) $\phi=90$ degrees

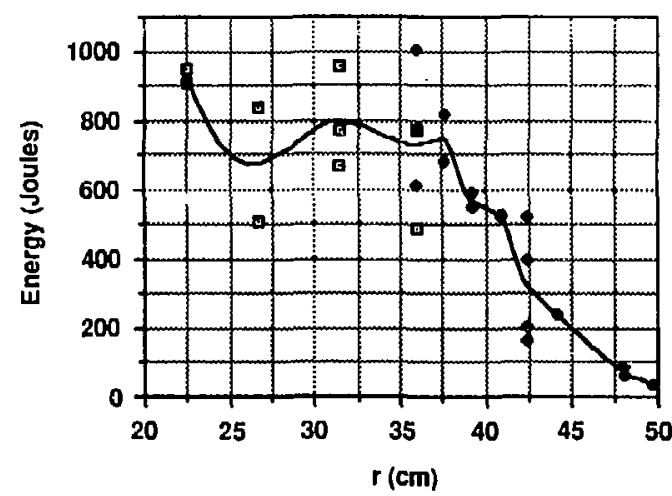

(c) $\phi=60$ degrees

$\vec{\omega}$

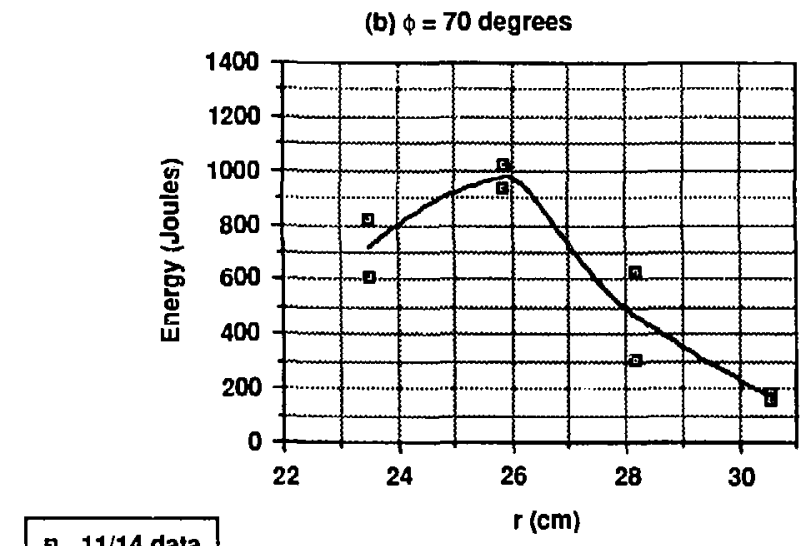

(d) $\phi=\mathbf{5 0}$ degrees

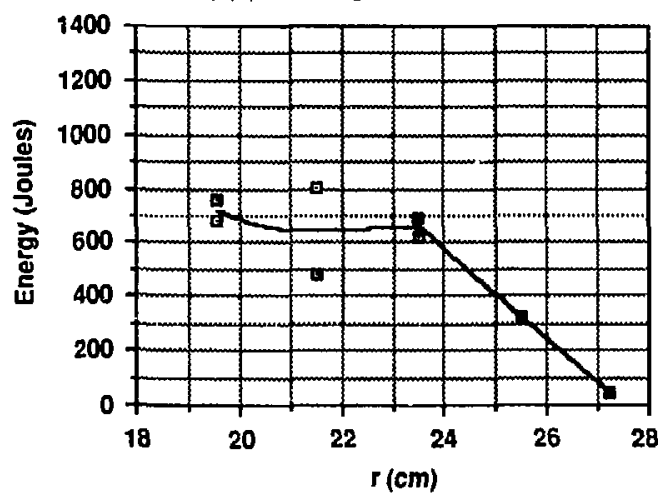

Figure 4.18. Radial energy loss versus orobe midplane radius. 


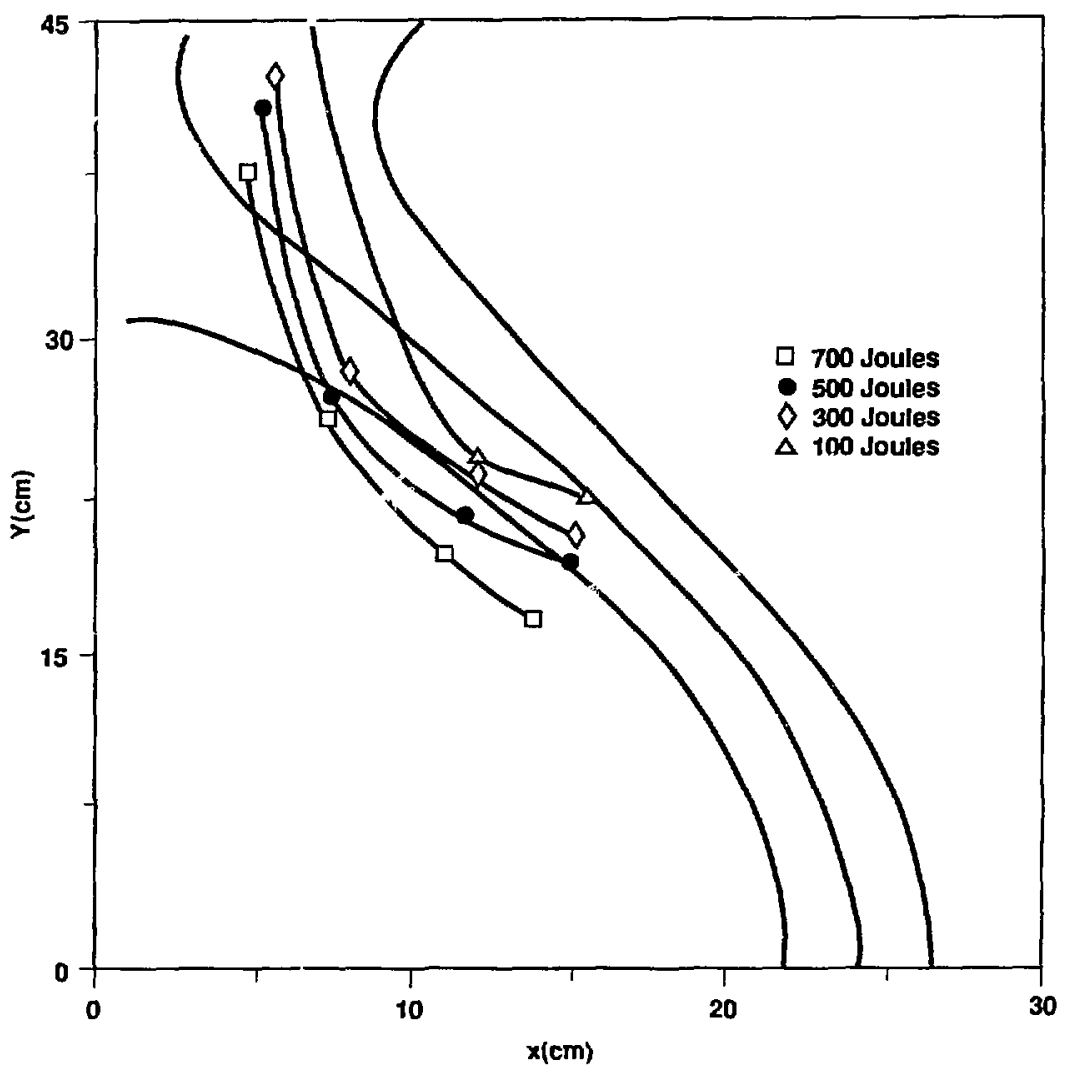

Figure 4.19. Comparison of the measured radial loss spatial structure with the calculated magnetic-curvature drift orbits. 
radial loss fraction is also quite small, around $7 \%$. However, as the magnetic field is swept down through the second-harmonic resonance, the results show distinct changes depending on which power system is operated. The $10-\mathrm{kG}$ system is the less efficient driver of the radial loss power, with a peak loss fraction of about $20 \%$ occurring at the same nagnetic field value as the axial loss and confinement peaks, $\frac{\Delta B}{B_{0}} \approx-1.5 \%$. On the other hand, the $5-\mathrm{kG}$ system drives the radial loss more efficiently, and the peak loss occurs at a lower magnetic field setting. In this case the peak value is $40 \%$ at $\frac{\Delta B}{B_{0}} \approx-3 \%$. When both ECRH systems are operated together, there is little synergistic effect: the radial loss fraction is roughly the weighted average of the two systems operated independently.

We expect the 5-kG ECRH to be dominant in driving the loss plume monitored by HEED probe, as this plume is strongly powered by the first-pass 5-kG absorption. The $10-\mathrm{kG}$ system, which is absorbed at the second-harmonic resonance by cavity heating alone, should be less effective, as observed. The opposite plume would be expected to exhibit the opposite behavior because it is powered mostly by cavity heating.

As $\frac{\Delta B}{B_{0}}$ is reduced, the super-hot plasma becomes annular, and its line density decreases. Therefore, it traps less of the ECRH power, leaving more power available to heat the hot-component plasma. In terms of the cavity-heating model, the decreasing line density of the super-hot component leads to decreased effective absorptive area. The hot-component thus traps more power, and the radial losses increase.

\subsubsection{Radial Confinement}

The radial energy confinement time can be calculated from the measurements of stored energy and radial power loss. Immediately after the ECRH is turned off, a fraction of the diamagnetism decays with a time constant of about 5 ms. Thus, the hot-component energy confinement time is $5 \mathrm{~ms}$ in the absence of velocity space diffusion processes associated with the ECRH. The rapidly decaying component of the diamagnetism is $M_{w a r m} \approx 5 \times 10^{4} \mathrm{Acm}$. Taking the hot-electron length as $120 \mathrm{~cm}$, this corresponds to a stored energy of $W_{\text {warm }} \approx 300 \mathrm{~J}$. Just before the ECRH is turned off, HEED probe measurements give radial loss powers of 
a) Average radial loss temperature

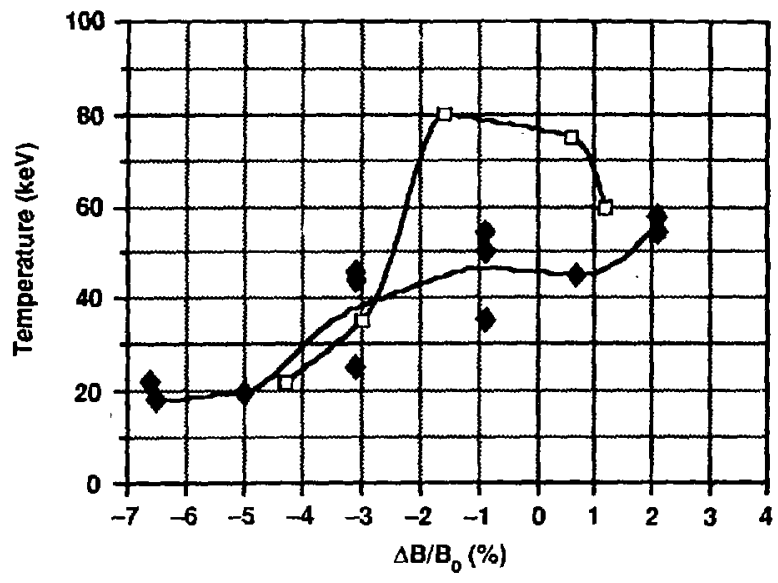

D 5-kG ECRH modulation

10-kG ECRH modulation

b) Radial loss fraction

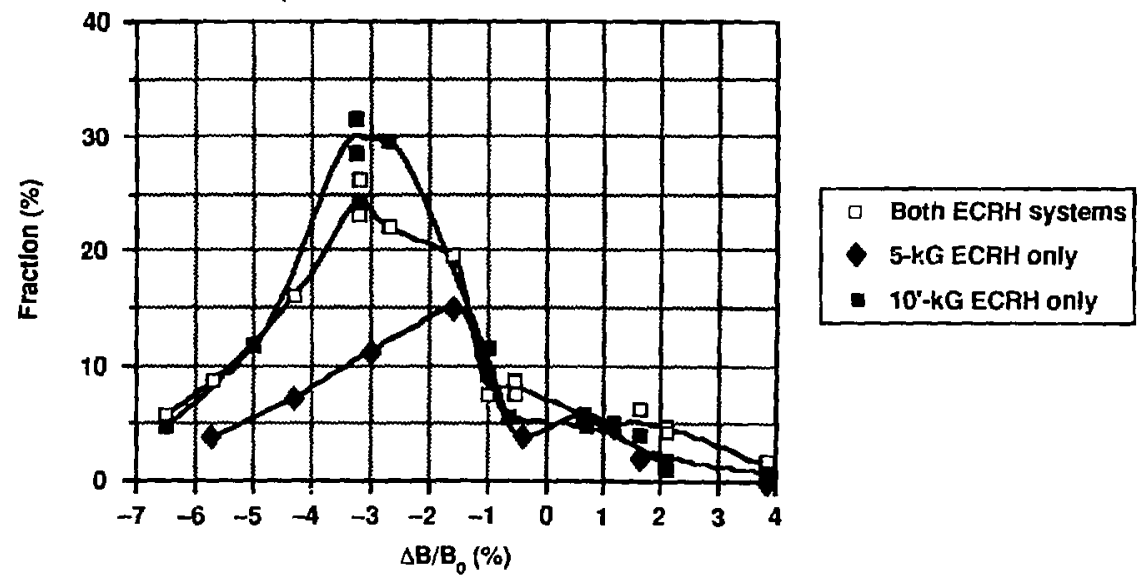

Figure 4.20. Variation of radial loss parameters with $\frac{\Delta B}{B_{0}}$ : (a) temperature, (b) power. 
about $22 \mathrm{~kW}$. We estimate the total loss power by multiplying this number by 1.4 to account for losses into the other loss plume, giving typical total loss power of $P_{\text {rad }} \approx 30 \mathrm{~kW}$. Therefore, during ECRH the radial confinement time is $\tau_{E}=$ $W_{\text {warm }} / P_{\text {radial }} \approx 10 \mathrm{~ms}$, assuming that the warm component is lost entirely by radial processes. The measurements suggest that the ECRH improves the energy confinement in the halo.

The measured halo confinement times are smaller than expected from classical pitch-angle diffusion into the loss cone. We can use eq. 4.19 to estimate of the collisional confinement time for the halo region. In this case, the cold-plasma density is small, so

$$
\tau_{E}=1.7 \frac{T_{\text {hot }}^{3 / 2}}{n_{\text {hot }}} \approx 28 \mathrm{~ms}
$$

where we have taken the electron temperature $T_{\text {hot }}$ as $30 \mathrm{keV}$, and the density $n_{\text {hot }}$ as $10 \times 10^{11} \mathrm{~cm}^{-3}$. The super-hot component electrons are too energetic to contribute to collisional scattering, and the hot-component electrons simply scatter on each other and the ions. The measured confinement times are 3-6 times smaller than this estimate.

\section{Fokker-Planck Calculations}

The SMOKE Fokker-Planck code was used to model the effects of radial losses on the plasma equilibrium and confinement. The code calculates the time evolution of the electron velocity space distribution function, including the effects of both collisional and ECRH-driven diffusion. The simulation is carried out along a single magnetic-flux tube ${ }^{34}$. To simulate the loss of electrons on open drift surfaces, an additional loss rate is added to a specific region of velocity phase space. The drift-losi rale $\nu_{d}$ is defined by

$$
\nu_{d}(w, \theta)=\left\{\begin{array}{ll}
\nu_{0} w, & \text { if } \theta_{0}-\Delta \theta \leq \theta \leq \theta_{0}+\Delta \theta \\
0, & \text { otherwise; }
\end{array} .\right.
$$

where $w$ is the electron energy, and we take $\theta_{0} \approx 60^{\circ}$ in correspondence to the pitch angles of open drift surfaces that connect to the plasma boundary. $\Delta \theta$ is a sensitive. function of spatial position at the plasma boundary; two values, $5^{\circ}$ and $10^{\circ}$, were used in simulations. The magnetic-curvature drift velocity was calculated by the SPLINE code to be $v_{d 0} \approx 1.5 \times 10^{5} \mathrm{~cm} / \mathrm{s}$ for electrons of energy $w_{0}=100 \mathrm{keV}$ 


\begin{tabular}{|c||ccc|ccc|}
\hline Run & $\Delta \theta$ & Fuel $\left(\frac{10^{13}}{\mathrm{~cm}^{3} \mathrm{~s}}\right)$ & Field $\left(\frac{\mathrm{V}}{\mathrm{cm}}\right)$ & $n_{e}\left(\frac{10^{21}}{\mathrm{~cm}^{3}}\right)$ & $T_{e}(\mathrm{keV})$ & $\tau_{E, \text { drift }}(\mathrm{ms})$ \\
\hline 1 & $5^{\circ}$ & 6 & 600 & 4.8 & 39 & 12.0 \\
\hline 2 & $5^{\circ}$ & 10 & 300 & 4.5 & 32 & 7.5 \\
\hline 3 & $5^{\circ}$ & 15 & 200 & 6.5 & 38 & 8.2 \\
\hline 4 & $10^{\circ}$ & 30 & 200 & 10.1 & 40 & 8.6 \\
\hline
\end{tabular}

Table 4.2. Summary of Fokker-Planck code results.

drifting near the sefaratrix. The drift velocity varies linearly with particle energy $w$. Electrons must drift some distance $l_{d}$ before they can be considered lost on the open drift surfaces; we take this to be one-half of the plasma circumference, $l_{d}=\pi(a+b) / 2$. Thus, the loss rate is proportional to particle energy with the constant given by

$$
\nu_{0}=\frac{v_{d 0}}{w_{0} l_{d}} \approx 40 / \mathrm{keV} / \mathrm{s} .
$$

A low energy cut-off is used to account for the lack of dominance of curvature drift on low-energy particles (see appendix A); $\nu_{0}$ goes to zero for $w<5 \mathrm{keV}$.

Several runs were carried out for varying amounts of ECRH electric-fieid strengths, fueling levels, and loss region widths; the results are summarized in table 4.2. Only the contribution of second- and higher-harmonic heating terms was considered in calculating the ECRH diffusion coefficients; fundamental ECRH was not included. ECRF heating was modeled by using two sources: an axially localized source with a Gaussian profile to model the 5-kG beam, and an axially uniform source with a smaller (17\%) electric-field strength to model cavity heating. The nominal peak electric-field strength of the $5-\mathrm{kG}$ beam is $600 \mathrm{~V} / \mathrm{cm}$, but this number must be divided by two to take into account the coupling of the gyrating electrons to the beam. Fueling was simulated with a fixed current of 3-keV electrons, presumably as created by fundamental heating. The initia] velocity distribution was a mirror-confined Maxwellian with a density of $1 \times 10^{11}$ $\mathrm{cm}^{-3}$ at a temperature of $30 \mathrm{keV}$. All simulations were run to equilibrium.

The final density of the electron distribution could be adiusted by varying the fuel source, while the average energy of the distribution is fixed at $30-40 \mathrm{keV}$ by the intersection of the second-harmonic heating characteristic with the driftloss region. The momentum phase space distribution for run 4 (see table 4.2 ) is 
shown in figure 4.21. The pitch-angle distribution is similar to that obtained in section 3.1.4. Energy confinement is indeed degraded from the classical values; the code predicts $\tau_{E, d r i f t} \approx 8 \mathrm{~ms}$ for nominal electric-field strengths. Essentially all of the losses are produced by the radial mechanism. Equilibrium density is $10 \times 10^{11}$ at an average energy of $40 \mathrm{keV}$. Thus, the Fokker-Planck calculation predicts equilibria with parameters similar to the experimental values.

Others have done detailed modeling of the buildup of energetic electrons with second-harmonic ECRH. For spatially localized second-harmonic ECRH at normal incidence, relativistic detuning limits the electron energies to below $20 \mathrm{keV}^{35}$. However, if cavity fields can provide heating at a wide range of magnetic-field values, electrons can gain energy at higher harmonics and a runaway tail is produced, the super-hot-electron component ${ }^{36,24}$. It is interesting to note that few runaway electrons were produced in the halo simulations that include the radial drift-loss mechanism. The drift-loss region of phase space blocks the diffusion of particles into regions of plase space where they can absorb higher harmonic ECRF. Thus, the phase space configuration of the halo favors buildup of the hot-electron component.

\section{Electron Radial Transport}

The halo region exists inside the separatrix; nevertheless radial losses are dominant from this rcgion. A calculation of the electron radial transport rate indicates that electrons in the halo region can move rapidly onto the open drift surfaces. The transport is driven by collisional small-angle scattering. Particles with different pitch angles have significantly different drift orbits, and radial transport results. This process is termed nonresonant neoclassical radial transport, and has веen examined theoretically for tandem mirrors, although for different circumstances 37, 38. A Lagrangian formalism is used based on the work of Cohen ${ }^{39}$. The analysis is limited to electron-ion collisions for simplicity; details of the calculation can be found in appendix $B$.

The calculation gives the radial flux of electrons in terms of the end-cell midplase radius, $r_{0}$, temperature, and density:

$$
\Gamma_{e i}=3.5 \times 10^{-2} \nu_{e i} n_{e} \frac{r_{0}^{2}}{l_{p}}\left[F_{1}\left(n_{e}, T_{e}, r_{0}\right)+F_{2}\left(n_{e}, T_{e}, r_{0}\right)\right],
$$




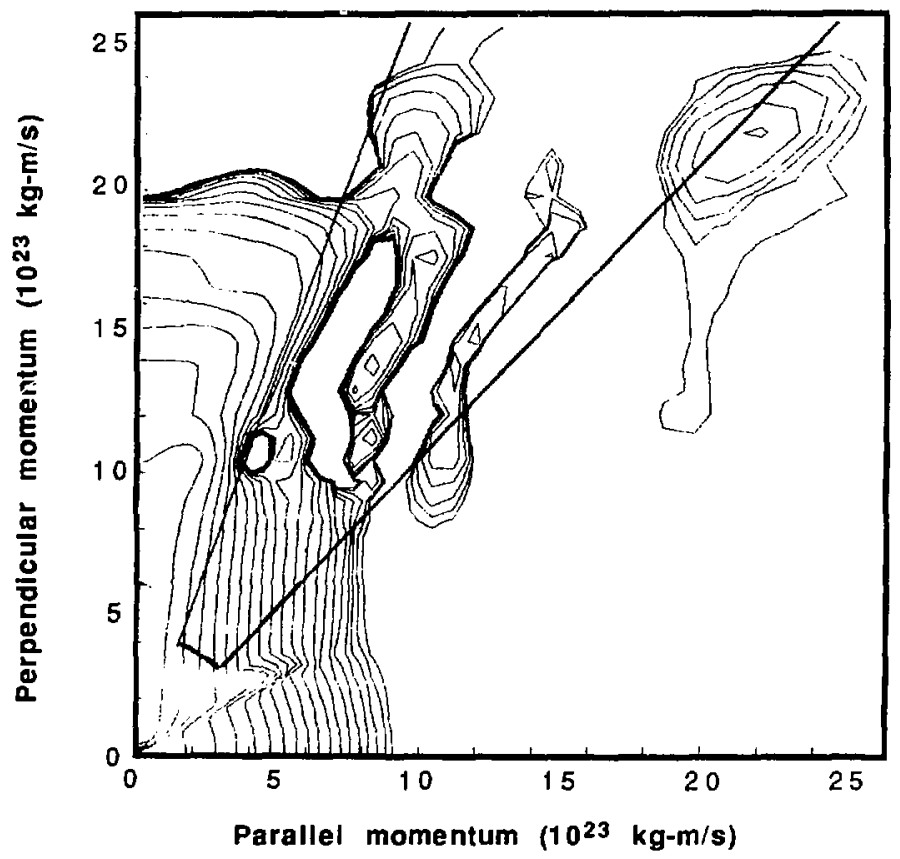

Figure 4.21. Fokker-Planck calculation of the halo electron distribution function. 
where $l_{p}$ is the pressure-gradient scale length

$$
l_{p} \equiv \frac{1}{n T_{e}} \frac{\partial\left(n T_{e}\right)}{\partial r_{0}}
$$

and $\nu_{e i}$ is the electron-ion collision frequency,

$$
\nu_{e i}=\frac{4 \pi n_{e} e^{4} \log \Lambda}{m^{1 / 2} T_{e}^{3 / 2}} \approx 730 \frac{n_{e}}{T_{e}^{3 / 2}} .
$$

In the last expression, we take $n_{e}$ in units of $10^{11} \mathrm{~cm}^{-3}$ and $T_{c}$ in $\mathrm{keV} . F_{1}$ and $F_{2}$ are functions of the electron temperature and density, as well as midplane radius (see appendix $B$ ). This estimate includes only the transport due to electronion scattering; to estimate the total flux, we multiply by two. Defining the radial particle velocity by $\Gamma \equiv n_{e} v_{r a d} \approx 2 \Gamma_{e i}$, we obtain

$$
v_{r a d} \approx 3.3 \times 10^{4} \frac{n_{e}\left[F_{1}\left(n_{e}, T_{e}\right)+F_{2}\left(n_{e}, T_{e}\right)\right]}{l_{p} T_{e}^{3 / 2}} .
$$

We can estimate the radial transport time as $\tau_{\text {rad }} \approx l_{\text {halo }} / 2 v_{\text {rad }}$ where the average width of the halo annulus $l_{\text {halo }} \approx 6 \mathrm{~cm}$. Taking $l_{p} \approx l_{\text {halo }} / 2$, the density as $10 \times 10^{11} \mathrm{~cm}^{-3}$, the temperature as $30 \mathrm{keV}$, and the average radius as $25 \mathrm{~cm}$, we get $\tau_{\text {rad }} \approx 2.5 \mathrm{~ms}$. We conclude that radial diffusion in the halo region is considerably faster than the loss rate via the open drift surfaces, estimated by the Fokker-Planck code to be about $8 \mathrm{~ms}$. The radial transport connects the entire halo region to the open drift surfaces. The overall radial confinement time can be estimated as the sum of the radial-transport time with the Fokker-Planckcalculated drift-loss time. This gives $\tau_{E} \approx 11 \mathrm{~ms}$ which is in good agreement with the experimental estimates.

\subsection{ECRH Power Balance}

\subsubsection{Temporal Variation}

The time behavior of the various terms of the power balance are shown in figures 4.22(a-e). For this data, the 5-kG ECRH was modulated, which affects all of the terms of the power balance to varying degrees. The absorbed power, shown in (a), should be balanced by the confined power (b), axial loss power (c), and radial loss power (d); all of these terms have been described in detail previously. 
The missing power, deternined by subtracting the sum of the confined, axial, and radial terms from the absorption, is shown in figure $4.22(\mathrm{e})$. Although noisy, the average value of the missing power is zero during the majority of the discharge. Only during the last few milliseconds, after the 5-kG ECRH turns of, is there significant missing power. During this period, the fundamental ECRH is well absorbed, but it does not contribute to stored energy or radial loss. In fact, the radial loss power begins to decay as soon as the $5-\mathrm{kG}$ power turned off, corresponding to the usual loss of the hot-electron component. Poor late-time power accountability of the 10-kG ECRH is typical, particularly after the 5-kG system is shut down. The 10-kG ECRH apparently drives a poorly-confined plasma component that has not yet been characterized.

\subsubsection{Variation with Vacuum Magnetic Field}

The behavior of the various power balance terms is shown as a function $\frac{\Delta B}{B_{0}}$ in figures $4.23(\mathrm{a}-\mathrm{c})$. The ECRH modulation was used to separate the actions of the two heating systems. When both ECRH systems are operated together, power balance is achieved over the entire range of field offset; note that the overall accuracy of the power balance is about $\pm 15 \%$. The curves labeled "Accounted" are the sum of confined, axial, and radial terms. The peaking near $-1.5 \%$ is due to efficient energy storage in the super-hot electrons. As the field is decreased, the absorption remains high due to increasing hot-component absorption, as evidenced hy the increasing radial loss. For field offsets below $-3 \%$, the second-harmonic resonance is no longer present in the 5-kG beamline, and power accountability deter urates. The plasmo formation process and structure under such conditions have not been well characterized. For positive field offsets, the absorption and losses steadily decrease as the hot-electron buildup is suppressed. The power balance is similar for independent operation of the 5-kG system. The trade-off of dominance between the super-hot and hot-component absorption is even more apparent, as the $5-\mathrm{kG}$ system couples more efficiently to the energetic electrons. For the 5 -kG ECRH, power balance deteriorates at both low and high field offsets. The discrepancy at slightly positive field offset may be associated with the formation of the relatively high-density, narrow super-hot-component plasma, as described in section 3.3.3. 
(a) Absorbed power

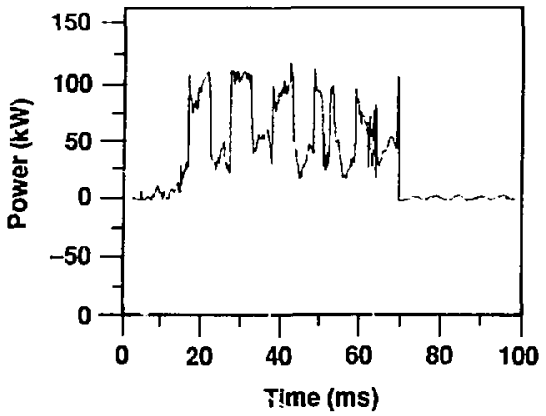

(c) Axial loss poiver

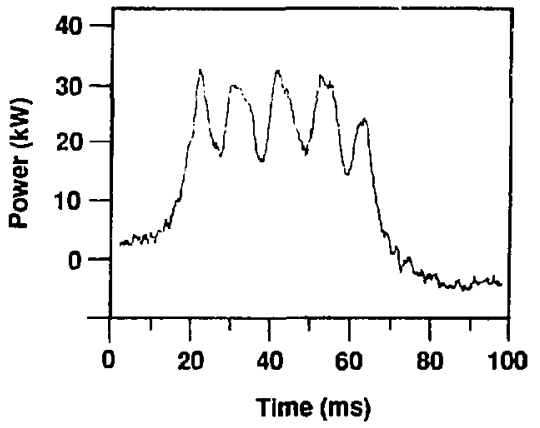

(b) Confine 1 power

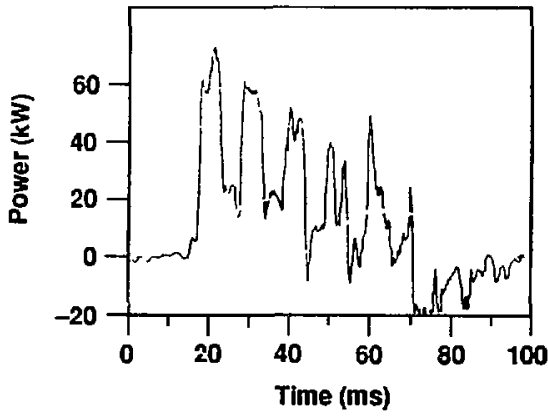

(d) Radial loss power

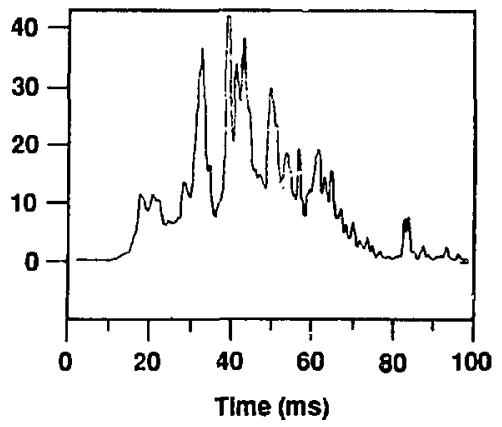

(e) Missing power

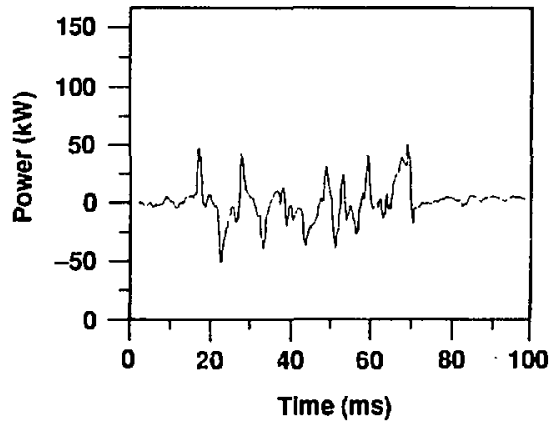

Figure 4.22. Temporal variation of the power-balance terms: (a) absorbed power, (b) confined power, (c) axial-loss power, (d) radial-loss power, (e) missing power. 
The power balance for independent operation of the fundamental is generally poor. Only under optimal operating conditions, $\frac{\Delta B}{B_{0}} \approx-1.5 \%$, can we account for all of the 10-kG power. Under all other conditions, the power balance is poor. Again, the 10-kG heater appears to couple to a poorly confined species whose loss is not measured. 


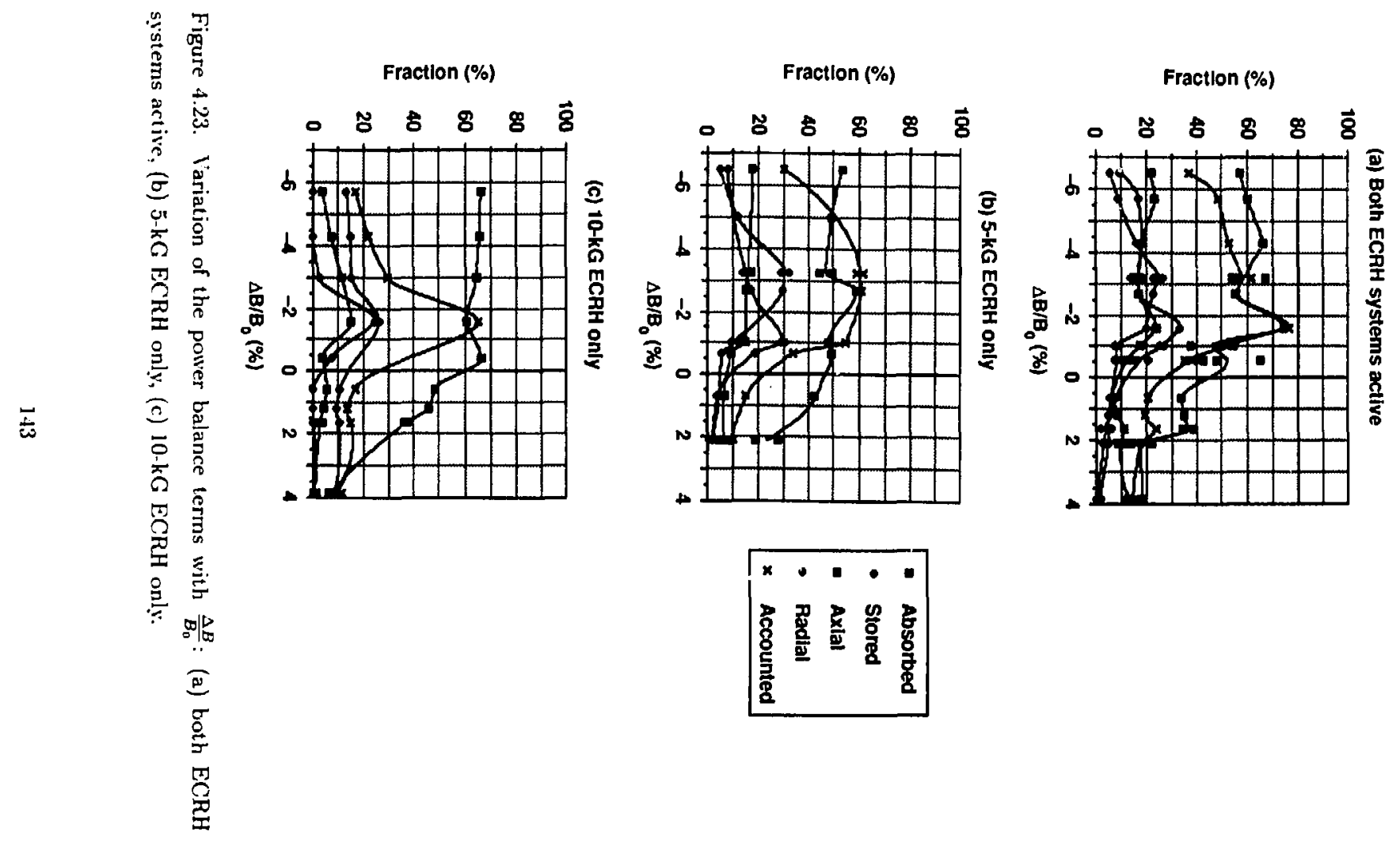




\section{Chapter 5}

\section{Conclusions}

\subsection{Hot-Electron Plasma Formation}

The ECRH-generated plasma in the West end-cell consists of two spatially distinct regions. The outer region is the halo, an elliptical annulus containing a large density of hot-component electrons and a smaller density of super-hot electrons. Within the inner boundary of the halo is the core plasma. The core contains a lower density of super-hot electrons and essentially no hot-component electrons. Instead, the core density is dominated by cold- and warm-component electrons. The plasma structure characteristics are summarized in tables 3.1 and 3.2 .

The early buildup of the hot-electron structure occurs in less than $10 \mathrm{~ms}$. The plasma initially forms along the magnetic axis at the location of the vacuum second-harmonic electron-cyclotron resonance. The plasma density and temperature simultaneously increase, causing a diamagnetic depression that shifts the second-harmonic resonance to the plasma boundary. Thus, the plasma grows outward. After a few milliseconds, the expansion is limited by the poor confinement associated with open magnetic-curvature drift surfaces that connect to the plasma boundary.

After the plasma expansion is complete, the plasma structure separates into the halo and core regions. The magnetic-field depression caused by the superhot-electron component removes the second-harmonic resonance from the core region. Heavily fueled by the center-cell gas box but deprived of an efficient power source, the core region becomes dominated by the less energetic warm- and cold-component electrons. In the halo region, the second-harmonic resonance is present, and a large density of hot-component electrons builds up. In particular, strong first-pass heating by the 5-kG ECRH beam creates a very sharp pressure 
gradient where the beam intersects the plasma. Electrons generated here drift on surfaces of different eccentricity, depending on their pitch angle. The pitch-angle distribution of hot- and super-hot electrons thus "fills in" the elliptical halo region as they drift. The worst-case separatrix between open and closed curvature-drift orbits determines the halo boundary.

\subsection{Hot-Electron Plasma Confinement}

ECRH power in TMX-U is absorbed principally near the second-harmonic resonance. The majority of the power is provided by first-pass absorption of the 5-kG ECRH, but a significant fraction is absorbed by cavity heating, particularly late in the discharge. Power from the $10-\mathrm{kG}$ heater is absorbed largely by cavity heating at the second-harmonic resonance. ECRF absorption at the fundamental is weak; only $10 \%$ of the total incident ECRH is absorbed. Fundamental heating generates warm-component electrons only and does not directly create hot- or super-hot electrons. The ECRF heating mechanism is descrived in detail in section 4.1.5.

The absorbed ECRF energy is stored principally in the well-confined surerhot-component electrons. During the initial portion of a discharge, these electrons trap a large amount of the ECRF power. Subsequently, their contribution to the absorption drops, first because their energy distribution is distorted by the strong second-harmonic ECRF, reducing the absorption coefficient, and later because of competition from the hot-component-electron population.

Electrons in the core region are lost axially. During the brief hot-electron buildup, electron end-loss spectra show a single temperature of about $10 \mathrm{keV}$. Later, during the equilibrium portion of the discharge, the spectrum is dominated by the 3-keV warm-component electrons, and hot-electron loss rates indicate a negligible core hot-component density. During this latter period, about half of the axial-loss power is provided by warm electrons that are lost by classical pitchangle diffusion into the mirror loss-cone.

The hot-component electrons are lost radially from the halo region. The electrons are heated at the beta-shifted second-harmonic resonance near the plasma boundary. Curvature drift causes the electrons to precoss away from the 5-kG beam. As they drift, some of the electrons scatter onto open drift surfaces and 
are lost, while others continue to drift on closed orbits. Electrons within the separatrix can undergo nonresonant neoclassical radial transport and be lost on the open drift orbits. Measured energy confinement i:mes agree well with theoretical estimates that combine Fokker-Planck simulations of the the drift-loss mechanism with calculations of the radial transport rate.

\subsection{Implications for Thermal-Barrier Operation}

In general, the hot-electron population created by the ECRH was not appropriate for thermal-barrier formation. In the core region, the mirror-trapped density is dominated by the $3-\mathrm{keV}$ (warm-component) electrons, rather than the desired 30-keV (hot-component) electrons. Although the warm-component electrons are sufficiently well confined to create the barrier potential, their loss rate is too high to allow creation of the high density originally proposed with a practical amount of ECRH power. The power required is given by

$$
P \approx \frac{n_{e} T_{e} V}{\tau_{E}}
$$

where $V \approx 1.5 \times 10^{5} \mathrm{~cm}^{3}$ is the plasma volume. For the measured (classical) energy-confinement time and temperature of the warm-component electrons, about $700 \mathrm{~kW}$ of ECRH power would be required to maintain a density of $5 \times 10^{12}$ $\mathrm{cm}^{-3}$. Such a scheme is therefore quite inefficient. In fact, the core-region electron temperature and density are too low because of ineffective heating. The necessary power from second-harmonic heating is not provided to the core region becaus? of the plasma diamagnetism associated with the low-density super-hot electror.s.

On the other hand, the plasma temperature and density in the halo regior, are much closer to the proposed values. In the presence of effective second-harmonic heating, the halo temperature and density build up to useful values desjite the less-than-classical confinement resulting from the radial loss process. Thus, it may be possible to create a high core density of $30-\mathrm{keV}$ electrons by somehow localizing the second-harmonic ECRF in the core region despite of the plasma diamagnetism. Two methods could be used to achieve this. First, the midplane magnetic field could be varied to create a "plasma synchrolron," as was advocated by J. F. Clauser in early 1986 but never attempted. This method is analogous to 
feedback control schemes used in tokamaks, where the vertical component of the confining magnetic field is modified to maintain force equilibrium as the plasma beta varies. Alternatively, the ECRH source frequency could be varied to achieve the same effect. As high-power tunable-frequency sources are not yet available, the frequency sweep could be accomplished using a number of sources, each tuned to a different frequency. The frequency sweep would be approximated by switching the sources on and off in sequence.

We expect that the problems associated with the diamagnetic shift of the second-harmonic resonance to be less severe or absent in an axisymmetric thermalbarrier configuration. Such a configuration is employed in the Gamma-10 experiment in Japan ${ }^{42}$. It would be useful to compare the hot-electron physics data from Gamma-10 with the results presented in this work.

The observed hot-electron plasma structure could impair the MHD stability of the center-cell. The beta of the warm-component electrons is not effective, because it occurs in the halo region on field lines that do not connect to the center-cell. Only the fraction of super-hot electrons present in the core region could provide a stabilizing influence. However, the super-hot electrons rotate too rapidly to be well-coupled to MHD phenomena in the center-cell, so they can improve stability only by increasing the normal curvature in the end-cell. As a consequence, the center-cell may be unstable ${ }^{43}$.

The fundamental ECRH appears to degrade the confinement of electrons, and in this fashion it accomplishes the desired goal of creating the positive plug potential. This is not the optinal method for potential formation, for it wastes ECRH energy. It would be more desirable for the fundamental heating to reduce the local electron confinement at the $10-\mathrm{kG}$ point by trapping the electrons more deeply within the mirror, thus fueling the barrier hot-electron population and making more effective use of the ECRF power. Furthermore, the fundamental power is deposited nonuniformly across the plasma cross section. The heating asymmetry could produce azimuthal potential variations, leading to radial transport of center-cell ions ?. 


\subsection{Suggestions for Future Work}

The results presented here are new and in many ways preliminary. Additional analysis of the existing data base would be useful, and further experiments could be carried out to test our theories of hot-electron plasma formation and confinement. Also, some of the data suggest new avenues for magnetic-mirror research.

There are literally gigabytes of data in the TMX-U archives that have never been examined in detail. Much could be learned from a judicious analysis of this data base. For example, a different $10-\mathrm{kG}$ heater-a Vlasov reflector-was used during 1984 and 1985. The radiation pattern from this antenna was much more compact, so it should be much more efficiently absorbed at the fundamental resonance. Comparison of $10-\mathrm{kG}$ heating data for the two different radiators could provide additional understanding of fundamental ECRH. Another area that bears exploration is the difference between West and East end-cell operation. The 5-kG beam in the East end-cell has the same orientation as in the West end-cell, but the curvature-drift surfaces are rotated by $90^{\circ}$. We therefore expect a different halo plasma structure in this end-cell, and relevant data may be available in the existing data base.

Several experiments could be carried out to test the plasma formation and structure models. For example, the $\frac{\Delta B}{B_{0}}$ scan experiment suggests that the superhot plasma becomes annular when the second-harmonic resonance is moved off the $z$ axis. We could confirm this result by repeating the limiter-probe scans at several different values of field offset. As another experiment, we could explore the radial transport process by varying the currents in the transition-region coil set. In particular, if this coil set is completely deactivated, the open drift surfaces should disappear, and radial electron loss from the halo should cease. Under such conditions the plasma spatial structure may be quite different, and the differences could be explored with additional limiter-probe experiments. Furthermore, by continuously varying the transition coil currents, we could measure the radial transport rates for different drift-orbit eccentricity functions, allowing a detailed comparison with the radial transport theory. Finally, the results obtained in this work should be verified during more conventional thermal-barrier operation in which the sloshing-ion beams are activated. 
The restilts obtained in this work also suggest some new areas of magneticmirror research. First, recall that a narrow, relatively high-density $\left(8 \times 10^{11}\right.$ $\mathrm{cm}^{-3}$ ) plasma is produced when the midplane magnetic fiel $\mathrm{H}$ is slightly elevated (see section 3.3.3). This "compact high-energy plasma" was originally reported by Failor ${ }^{18}$. Such a plasma formation is very interesting, because it is closer to the desired core hot-electron plasma structure than that achieved during any other operation. The study of this phenomenon could lead to a new method for controlling the dominance of the super-hot electrons in an ECRH-driven magnetic mirror. In a similar vein, the Fokker-Planck simulations of the halo plasma suggest another method for controlling the super-hot electrons. In the halo region, hot-electron thermal runaway is blocked by the lossy region of phase space introduced by the open drift surfaces. It is possible that a similar velocity-space configuration could be created artificially to induce the same effect. Specifically, the second-harmonic ECRH could be applied in an axially short, shallow magnetic well imbedded within a more typical mirror. The mirror ratio of the inner mirror would be selected to produce a velocity-space configuration similar to that produced by the drift-loss mechanism. Specifically, we want $\theta_{c} \approx 60^{\circ}$, requiring a mirror ration $R \approx 1.3$. However, as the hot-electrons are driven out the inner mirror by the strong ECRH velocity-space diffusion, they would not be lost but would instead transfer a useful fraction of their energy to other particles within the outer mirror, thus making efficient use of the ECRH power while controlling the hot-electron runaway problem. This concept would be amenable to detailed simulation with a Fokker-Planck code, and could be experimentally investigated at a smaller magnetic-mirror facility. 


\section{Appendix A}

\section{Computer Code Descriptions}

\section{A.1 The SPLINE Guiding Center Drift Code}

Charged particles confined in a tandem mirror undergo a combination of gyration, bounce, and drift motions. Generally, the electron-cyclotron motion occurs on a time scale much faster than the drifts, and the particle motion can be described in terms of guiding center drifts, obtained by averaging the equation of motion over the fast gyration time scale. The guiding center drifts are typically caused by a combination of magnetic curvature, magnetic gradients, and electric fields. Altogether, the drift motion at a single point in space is described by

$$
\frac{d \vec{x}}{d t}=\frac{m}{\mathrm{e}}\left(v_{\|}^{2}+\frac{1}{2} v_{\perp}^{2}\right) \frac{\overrightarrow{R_{c}} \times \vec{B}}{R_{c}^{2} B^{2}}+\frac{\vec{E} \times \overrightarrow{\mathbf{B}}}{B^{2}} .
$$

Here, we have assumed negligible plasma diamagnetism, so $\vec{\nabla} \times \vec{B}=0$, and $\vec{R}_{c}$ is the magnetic-field radius of curvature ${ }^{3}$.

For energetic electrons confined in a magnetic mirror, the bounce motion is also much faster than the drifts, so it is useful to calculate the bounce-averaged guiding center drift velocity:

$$
<\overrightarrow{v_{d}}>=\frac{\oint \frac{d \cdot}{v_{\|}} \overrightarrow{v_{d}}}{\oint \frac{d_{d}}{v_{\|}}} .
$$

In operation, the code follows a spatial ensemble of particles with a given midplane pitch angle. The bounce integral is calculated for each particle, yielding a vector field of drift velocities. This, in turn, is integrated in time from some given starting location to obtain the drift orbits.

Accurate calculation of the bounce-averaged drift velocities requires precisc inowledge of the magnetic-field topology. In the SS'LINE code, magnetic field 
lines, as generated by the MAFCO code for the TMX-U field case UPHP30, were converted into a three-dimensional look-up table. Tricubic splines were used on the resulting grid to obtain the various spatial derivatives of the magnetic field necessary to calculate the curvature-drift velocities.

Drift orbit calculations shown within this dissertation do not include electric field effects. For the energetic electrons in the halo, magnetic-curvature drift is dominant. The curvature-drift velocity component increases linearly with the particle energy, while the $\vec{E} \times \vec{B}$ drift velocity depends only on the local fields. In the halo, the curvature-drift velocity varies with pitch angle and position, but can be roughly estimated by

$$
v_{B} \approx 1500 \mathrm{w} \mathrm{cm} / \mathrm{s} \text {, }
$$

where $w$ is the electron energy in keV. The $\vec{E} \times \vec{B}$ drift velocity is

$$
v_{\vec{E} \times \vec{B}} \approx \frac{\phi}{l_{\phi} B_{0}},
$$

where $\phi$ and $l_{\phi}$ are the plasma potential and potential gradient scale length, respectively; and $B_{0}$ is the midplane magnetic field. The two drift velocities become equal when the electron energy is

$$
w_{3}=\frac{\phi}{7.5 l_{\phi}} .
$$

For typical ECRH-only discharges, $\phi<400 \mathrm{~V}$, and taking $l_{\phi}$ as half the plasma radius $(11 \mathrm{~cm})$, we get $w_{1} \approx 5 \mathrm{keV}$. Thus, the hot- and super-hot-component electrons should be insensitive to the $\vec{E} \times \vec{B}$ drift.

\section{A.2 The MECH Power Deposition Code}

The MECH code traces ray trajectories through a model plasma using the cold plasma dispersion relationship for a cold, collisionless electron plasma. Determination of the cold-plasma dispersion relation involves combining Maxwell's equations with the equations of motion for a single electron. The result can be expressed as

$$
\tilde{D} \cdot \vec{E}=\mathbf{0},
$$

where $\tilde{D}$ is the dielectric tensor. The dispersion relation is then given by

$$
D \equiv\|\tilde{D}\|=0 .
$$


Solution of eq. A.7 yields the Appleton-Hartree relation ${ }^{40}$.

In ray tracing, the wave electric field is written in the form:

$$
\vec{E}=\operatorname{Re}\left[\vec{E}_{0}(\vec{r}, t) e^{i \psi(\vec{r}, t)}\right],
$$

where the wave amplitude, $\vec{E}_{0}$, and phase function, $\psi$ are assumed to be slowly varying functions of space and time. This ansatz allows solution of the eikonal equation, and yields the ray trajectories from the equations:

$$
\begin{aligned}
& \frac{d \vec{r}}{d t}=-\frac{\partial D / \partial \vec{k}}{\partial D / \partial \omega}, \\
& \frac{d \vec{k}}{d t}=-\frac{\partial D / \partial \vec{r}}{\partial D / \partial \omega} .
\end{aligned}
$$

These two equations can be reduced to a system of simultaneous ordinary differential equations and solved numerically ${ }^{41}$.

As the ray propagates, the absorption is calculated using an expression derived from the fully-relativistic dispersion relationship in the weak-damping limit. The code calculates the hermitian part of the conductivity tenso $\tilde{j}^{H}$ to determine

$$
\frac{d S}{d s}=\vec{E}^{*} \cdot \tilde{\sigma}^{H} \cdot \vec{E},
$$

where $S$ is the magnitude of the Poynting vector, and $s$ is the arc length along a ray.

Computation of the fully-relativistic dispersion relationship is complicated. Briefly, the linearized Vlasov equation is combined with Maxwell's equations to yield the dispersion tensor, which contains the conductivity tensor. The terms of the conductivity tensor involve integrals over momentum space, and an infinite sum over harmonic number. Singularities associated with the resonance at each harmonic break the conductivity tensor into hermitian and anti-hermitian parts. The delta functions introduced by the singularities into the hermitian part facilitates the integration over momentum. What remains is an integration over pitch angle, which must be carried out numerically, and the sum on harmonic number ${ }^{10}$. In the $\mathrm{MECH}$ code, a finite number, usually less than 10 , of the harmonic terms are calculated. An isotropic, relativistic Maxwellian velocity distribution is assurned.

The MECH code can trace up to 200 rays during each run. Groups of rays can be launched to simulate the measured antenna patterns. For the $5-\mathrm{kG}$ simulation, 
an elliptical bundle of rays represent the Gaussian beam profile from the twist reflector, while the 10-kG uniform heater is simply modeled with a diverging, rectangular grid of uniformly weighted rays. The power deposited by the rays is recorded as a function of radial flux coordinates, yielding a power deposition profile that is displayed at the end-cell midplane.

The code uses a fairly sophisticated plasma model. Multiple electron populations can be specified by the user, each having one of several radial profiles for the temperature and density. MECH uses a long-thin model of the vacuum magnetic field, and keeps track of the field depression introduced by the plasma diamagnetism. 


\section{Appendix B}

\section{Electron Radial Transport}

We begin with a sin.plified kinetic equation,

$$
\frac{\partial f}{\partial t}=C_{J}(f)
$$

The equation is assumed to contain only a collisional time scale that is slow compared to the three constants of the motion:

$$
\begin{aligned}
& J_{1} \equiv \frac{\pi m v_{\perp}^{2}}{\omega_{\mathrm{cE}}}, \\
& J_{2} \equiv m \oint v_{\|} d s, \\
& J_{3} \equiv \frac{2 \pi q}{c}\langle\psi\rangle,
\end{aligned}
$$

where the angle brackets in the definition of $J_{3}$ specify an average over the gyration, bounce, and drift motion of the particle. Here, $\psi$ and $\gamma$ are the radial and azimuthal flux coordinates respectively, defined by $\vec{B}=\vec{\nabla} \psi \times \vec{\nabla} \gamma$, while $s$ is the distance along a field line. For the collision operator on the right hand side, we use the Lorentz operator:

$$
C(f)=\nu_{e i} \frac{v_{t h}^{3}}{v^{3}} \frac{\partial}{\partial \lambda}\left[\left(1-\lambda^{2}\right) \frac{\partial f}{\partial \lambda}\right],
$$

where $\lambda=v_{\|} / v$, and $\nu_{e i}$ is the electron-ion collision frequency. We proceed, following the Lagrangian formulation of reference [39]. The kinetic equation is integrated over $J_{1}, J_{2}$ and averaged over the (fast) non-collisional time scales to get

$$
\frac{\partial n_{3}}{\partial t}=-\frac{\partial \Gamma_{3}}{\partial J_{3}}
$$

The other derivatives involving $\partial / \partial J_{1}, \partial / \partial J_{2}$ do not survive the flux-surface average to leading order. The flux can be expressed as an integral in $E, \mu$ coordinates, 
where $E=m v^{2} / 2$, and $\mu=m v_{\perp}^{2} / 2 B$ :

$$
\Gamma_{3}=-\frac{4 \pi^{2} m^{3} e \nu_{e i} v_{t h}^{3}}{c} \int d E d \mu \frac{d s}{B} d \gamma \frac{(E-\mu B-\phi)^{1 / 2}}{(E-\mu B)^{3 / 2}} \mu \delta_{\mu}^{2} \frac{\partial f}{\partial J_{3}} .
$$

$\Gamma_{3}$ represents the flux of electrons transported radially across a drift surface. The transport is driven by the deviations of particle drift surfaces from magnetic flux surfaces, the magnitude of which is a function of pitch angle. As the electrons collisionally scatter in pitch angle, they move from drift surface to drift surface, thus undergoing radial motion; this is termed nonresonant neoclassical transport. $\delta_{\mu}=\partial \delta / \partial \mu$, and $\delta$ is the magnitude of the elliptical deviation of flux surfaces from the drift surfaces:

$$
J_{3} \approx \frac{2 \pi e}{c}(\psi+\delta \cos m \gamma),
$$

where $m=2$ in the TMX-U end-cell. $\delta$ is obtained by expanding $J_{2}$ in the neighborhood of a particular fiux surface, $\psi=\bar{\psi}$ :

$$
J_{2} \approx J_{2 s}+\frac{\partial J_{2 s}}{\partial \psi}(\psi-\bar{\psi})+\hat{J}_{2} \cos m \gamma
$$

and then using the drift equations of motion (see Cohen, et al. ${ }^{38}$ ). The result is

$$
\delta=\frac{\hat{J}_{2}}{\partial J_{2,} / \partial \psi} .
$$

In this particular problem, we use a parabolic approximation to the measured elliptical, hollow pressure profile to obtain the equilibrium magnetic field. Assuming a square-well axial magnetic-field profile to simplify the bounce integral,

$$
\delta=\epsilon^{\prime} \psi
$$

where $\epsilon^{\prime}=\frac{\epsilon^{2}-1}{\epsilon^{2}+1}$, with $\epsilon$ the eccentricity of the drift orbits, as calculated with the SPLINE code (see figure 3.14). As the eccentricity is a function of pitch angle alone, we change the integration variables to $E, \lambda$, and carry out the trivial integrations over the bounce motion and drift phase:

$$
\Gamma_{3}=4 r^{3} l_{b} \nu_{e i} v_{i}^{3} \omega_{c e} r_{0}^{4} \int \frac{d E}{E} d \lambda\left(1-\lambda^{2}\right) \epsilon_{\lambda}^{\prime 2} \frac{\partial f}{\partial J_{3}},
$$

where $l_{b}$ is the bounce length, and $r_{0}$ the midplane radius.

The assumption implicit in this analysis is that the collision frequency $\nu_{0}$ is less than the drift frequency. In the TMX-U end-cell halo, this requirement is satisfied 
by the majority of the electrons, but cooler electrons will tend to scatter before they complete a half precession. We treat this aspect of the problem by dividing the energy integral into two regimes separated by a cut-off energy defined by the equality of the drift and collision frequencies. In the halo, the drift frequency is given by

$$
v_{d}=\frac{v_{d}}{\pi r_{0}} \approx 210 \frac{E}{r_{0}},
$$

where $r_{0}$ is the average radius of the drift orbit, and $E$ is in keV. This calculation of the drift frequency is based on the vacuum field only. For the measured parameters of the TMX-U halo, plasma diamagnetism can increase the drift by as much as 40\%. However, the vacuum drift alone will suffice to generate an estimate of the radial transport flux. The appropriate collision firequency corresponds to pitchangle scattering from circular drift orbits, $\theta \approx 90^{\circ}$, to the most elliptical orbits, $\theta \approx 60^{\circ}$. Thus, $\nu_{0}$ is the $30^{\circ}$ angle-scatter frequency given by

$$
\nu_{0}=\frac{\nu_{\mathrm{ei}}}{\sin ^{2} 30^{\circ}} \approx 2.9 \times 10^{3} \frac{n}{E^{3 / 2}},
$$

with $E$ again in $\mathrm{keV}$, and $n$ in units of $10^{11} \mathrm{~cm}^{-3}$. Setting the two equal, we obtain the cutoff energy:

$$
E_{\mathrm{c}}=1.2\left(n r_{0}\right)^{2 / 5} \text {. }
$$

For electrons with smaller energies, the value of the eccentricity must be reduced by the ratio of the drift frequency to the collision frequency:

$$
\epsilon^{\prime} \rightarrow \frac{\nu_{\mathrm{d}}}{\nu_{0}} \epsilon^{\prime}=7.2 \times 10^{-2} \frac{E^{5 / 2}}{n_{\mathrm{e}} r_{0}} \epsilon^{\prime} .
$$

We can now complete the integrals over energy and pitch angle. The distribution function is assumed to be an anisotropic Maxwellian:

$$
f(E, \lambda)=\frac{n_{e}}{\pi^{3 / 2} v_{t}^{3}} e^{-E / T_{e} g(\lambda)} \frac{g}{\lambda_{1}}
$$

where the pitch-angle distribution is given by

$$
g(\lambda)=\left\{\begin{array}{ll}
1, & \text { if } 1 \geq|\lambda|>\lambda_{1} ; \\
0, & \text { if }|\lambda|<\lambda_{1}
\end{array},\right.
$$

with $\lambda_{1}=0.5$ (distribution is cutoff at a pitch angle of $60^{\circ}$ ) as an approximation to the measured pitch-angle distribution. A simple, piecewise paratolic fit to the 
eccentricity is used to complete the pitch-angle integral. We need the $J_{3}$ derivative of the distribution in terms of midplane radius:

$$
\frac{\partial f}{\partial r_{0}}=\frac{f}{4 \pi m \omega_{c e} r_{0}}\left(\frac{1}{l_{n}}+\frac{3}{2 l_{T}}-\frac{E}{T_{e}} \frac{1}{l_{T}}\right)
$$

where the gradient scale lengths are

$$
\begin{aligned}
& l_{n}=\frac{1}{n_{e}} \frac{\partial n_{e}}{\partial r_{0}}, \\
& l_{T}=\frac{1}{T_{e}} \frac{\partial T_{e}}{\partial r_{0}} .
\end{aligned}
$$

The flux becomes

$$
\Gamma_{3}=\pi^{1 / 2} m^{3} l_{b} r_{0}^{3} n \nu_{e i}\left(\frac{F_{1}\left(n_{e}, T_{e}, r_{0}\right)}{l_{n}}+\frac{F_{1}\left(n_{e}, T_{e}, r_{0}\right)}{l_{T}}\right) .
$$

The two functions $F_{1}$ and $F_{2}$ contain the two pieces of the energy integral,

$$
\begin{gathered}
F_{1}=0.58 \frac{T_{e}^{5 / 2}}{n r_{0}} \gamma\left(\frac{E_{c}}{T_{e}}, \frac{5}{2}\right)+E_{1}\left(\frac{E_{c}}{T_{e}}\right) \\
F_{2}=0.58 \frac{T_{e}^{5 / 2}}{n r_{0}}\left[\frac{3}{2} \gamma\left(5 / 2, E_{c} / T_{e}\right)-\gamma\left(7 / 2, E_{c} / T_{e}\right)\right] \\
+\left[\frac{3}{2} E_{1}\left(E_{c} / T_{e}\right)-\exp \left(-E_{c} / T_{e}\right)\right],
\end{gathered}
$$

where $E_{1}(x)$ represents the exponential integral, and $\gamma(a, x)$ is the incomplete gamma function.

Finally: we convert the form of eq. B.6 to radial coordinates. The result is

$$
\frac{\partial n_{e}}{\partial r_{0}}=-r_{0} \frac{\partial}{\partial r_{0}}\left(r_{0} \Gamma_{e i}\right)
$$

with the flux given by

$$
\Gamma_{e i}=\frac{n_{e} r_{0}^{2} \nu_{e i}}{8 \pi^{1 / 2}}\left[\frac{F_{1}\left(n_{e}, T_{e}, r_{0}\right)}{l_{n}}+\frac{F_{2}\left(n_{e}, T_{e}, r_{0}\right)}{l_{T}}\right] .
$$

Or, if we take the density and temperature scale lengths to be roughly equal, we can write the flux in terms of the pressure gradient scale length $l_{p}$,

$$
\Gamma_{e i}=\frac{n_{e} r_{0}^{2} \nu_{e i}}{16 \pi^{1 / 2} l_{p}}\left[F_{1}\left(n_{e}, T_{e}, r_{0}\right)+F_{2}\left(n_{e}, T_{e}, r_{0}\right)\right] .
$$

This expression is used in section 4.4 to estimate the radial loss rate. 


\section{Bibliography}

[1] D. E. Baldwin and B. G. Logan, Phys. Rev. Lett. 43, 1318 (1979).

[2] N. J. Fisch, Rev. Mod. Phys. 59, 175, (1987).

[3] F. F. Chen, Introduction to Plasma Physics (Plenum Press, New York, 1974), 64.

[4] J. B. Taylor, Plyys. : 'iuids 6, 1529 (1963).

\{5\} T. K. Fowler and B. G. Logan, Comments Plasma Phys. Contr. Fusion 2, 167 (1977).

[6] F. H. Coensgen, T. C. Simonen, A. K. Chargin, and B. G. Logan, TMX Upgrade Major Project Proposal, Lawrence Livermore National Laboratory, Livermore, CA, LLL-Prop-172 (1980).

[7] G. Dimonte, "Radial Transport in a Tandem Mirror," submitled to Phys. Rev. Lett. (January 1988).

[8] B. W. Stallard, "Uniform Heater," in TMX-U Final Report, Vol. 2, G. D. Porter, Ed., Lawrence Livermore National Laboratory, Livermore, CA, UCID-20981 (1988).

[9] B. W. Stallard et al., Foctusing Tuist Reflector for Electron-Cyclotron Resonance Heating in the Tandem Mirror Experiment-Upgrade, Lawrence Livermore National Laboratory, Livermore, CA, UCRL-90784 (1984), presented at $4^{\text {th }}$ International Workshop on ECE and ECRH, Frascati, Italy (1984).

[10] D. B. Batchelor, R. C. Goldfinger, and H. Weitzner, Phys. Fluids 27, 2835 (1984).

[11] R. A. James, "Hot-Electron Spatial Profiles," in Summary of TMX-U Results: 1984, T. C. Simonen, Ed., Lawrence Livermore National Laboratory, Livermore, CA, UCID-20274 (1984). 
[12] E. H. Silver and J. F. Clauser, Measuring the Fraction of Mirror-Trapped Hot-Electrons in a Thermal-Barrier Tandem-Mirror Machine, Lawrence Livermore National Laboratory, Livermore, CA, UCRL-95027 (1986), submitted to Nucl. Fusion.

[13] R. H. Cohen and L. L. LoDestro, ECRH-Heated Distributions in ThermalBarrier Tandem Mirrors, Lawrence Livermore National Laboratory, Livermore, CA, UCRL-97582 (1987), presented at the International School of Plasma Physics, Varenna, Italy, September 1-11, 1987.

[14] H. Enge, Introduction to Nuclear Physics (Addison-Wesley, Reading, MA, 1966).

[15] D. E. Gray, American Institute of Physics Handbook (McGraw-Hill, New York, 1972).

[16] A. B. Shapiro, Topaz2d - A Two-Dimensional Finite Element Code for Heat Transfer Analysis, Electrostatic and Magnetostatic Problems, Lawrence Livermore National Laboratory, Livermore, CA, UCID-20824 (1986).

[17] R. W. Stineman, "A Consistently Weli-Behaved Method of Interpolation," Personal Computing (November 1984).

[18] B. H. Failor, X-Ray Imaging Studies of Electron-Cyclotron Microwave-Heated Plasmas in the Tandem Mirror Experiment-Upgrade, Lawrence Livermore National Laboratory, Livermore, CA, UCRL-53717 (1986).

[19] X. Chen, B. G. Lane, D. L. Smatlak, R. S. Post, and S. A. Hokin, to be published in Phys. Fluids.

[20] R. A. James, C. J. Lasnier, and R. F. Ellis, Rev. Sci. Instrum. 57, 1968 (1986).

[21] W. C. Turner, in Summary of TMX-U Results: 1984, Vol. 1, T. C. Simonen, Ed., Lawrence Livermore National Laboratory, Livermore, CA, UCID-20274 (1984).

[22] T. C. Simonen et al., IEEE Trans. Plasma Science 16, 1 (1988). 
[23] G. D. Porter, M. R. Carter, J. H. Foote, R. S. Hornady, and the TMX-U Group, Axial Potential Profile Measurements in TMX-U, Lawrence Livermore National Laboratory, Livermore, CA, UCRL-94922 (1987), submitted to Nucl. Fusion.

[24] T. D. Rognlien, Y. Matsuda, and J. J. Stewart, "Modeling Electron-Cyclotron Resonance Heating in Tandem Mirrors," Phys. Fluids (in press).

[25] M. Bornatici, R. Cano, O. DeBarbieri, and F. Engelmann, Nucl. Fusion 23, $1153(1983)$.

[26] J. Schivell, G. Renda, J. Lowrance, and H. Hsuan, Rev. Sci. Instrum. 53, 1527 (1982).

[27] R. Horton, in TMX-U Final Report, Vol. 2, G. D. Porter, Ed., Lawrence Livermore National Laboratory, Livermore, CA, UCID-20981 (1988).

[28] A. W. Molvik, Rev. Sci. Instrum. 52, 704 (1981).

[29] E. B. Hooper, in Summary of TMX-U Results: 1984, T. C. Simonen, Ed., Lawrence Livermore National Laboratory, Livermore, CA, UCID-20274 (1984).

[30] J. H. Foote, G. W. Coutts, L. R. Pedrotti, G. Schlander, and B. E. Wood, Rev. Sci. Instrum. 56, 1117 (1985).

[31] D. Hill, "Measurement of the Electron End Losses" in TMX-U Final Report, Vol. 1, G. D. Porter, Ed., Lawrence Livermore National Laboratory, Livermore, CA, UCID-20981 (1988).

[32] D. L. Book, NRL Plasma Formulary (Naval Research Laboratory, Washington, D.C., 1983).

[33] L. Spitzer, Jr., Physics of Fully Ionized Gases, Second Revised Edition (Interscjence Publishers, New York, 1962).

[34] Y. Matsuda and J. J. Stewart, in Proceedings from the 10th Conference on Numerical Simulation of Plasmas, San Diego, CA (1983).

[35] B. W. Stallard, Y. Matsuda, and W. M. Nevins, Nucl. Fusion 23, 213 (1983). 
[36] T. D. Rognlien, Y. Matsuda, and J. J. Stewart, in Proceedings of the 6th Topical Conference on Radiofrequency Plasma Heating, Callaway Gardens, GA (1985).

[37] J. R. Myra, P. J. Catto, and R. D. Hazeltine, Phys. Fluids 25, 2028 (1982).

[38] A. A. Mirin et al., Nucl. Fusion 23, 703 (1983).

[39] R. H. Cohen, K. Hizandis, K. Molvig, and I. B. Bernstein, Phys. Fluids 27 377 (1984).

[40] T. H. Stix, The Theory of Plasma Waves, (McGraw Hill, New York, 1962).

[41] K. R. Audenaerde and J. E. Scharer, Nucl. Technology/Fusion 2, 623 (1982).

[42] T. Cho et al., "Potential Formation and Transport Studies on Tandem Mirror Gamma 10," Proceedings of the Eleventh International Conference on Plasma Physics and Controlled Nuclear Fusion Research, Kyoto, Japan, 1320 November 1986.

[43] T. C Simnnen, H. L. Berk, T. A. Casper, and C. Y. Chen, Low Frequency Stability Analysis of the Tandem Mirror Expernment-Upgrade, Lawrence Livermore National Laboratory, Livermore, CA, UCRL-97697 (1988), submitted for publication in Nucl. Fusion. 